Portland State University

PDXScholar

Narrowing the Cognitive Distance Between

Engineers and Customers: a Novel Approach, Based on Fuzzy Cognitive Mapping

Byung Sung Yoon

Portland State University

Follow this and additional works at: https://pdxscholar.library.pdx.edu/open_access_etds

Part of the Engineering Commons, and the Technology and Innovation Commons Let us know how access to this document benefits you.

Recommended Citation

Yoon, Byung Sung, "Narrowing the Cognitive Distance Between Engineers and Customers: a Novel Approach, Based on Fuzzy Cognitive Mapping" (2020). Dissertations and Theses. Paper 5412. https://doi.org/10.15760/etd.7285

This Dissertation is brought to you for free and open access. It has been accepted for inclusion in Dissertations and Theses by an authorized administrator of PDXScholar. Please contact us if we can make this document more accessible: pdxscholar@pdx.edu. 
Narrowing the Cognitive Distance Between Engineers and Customers:

A Novel Approach, Based on Fuzzy Cognitive Mapping

by

Byung Sung Yoon

A dissertation submitted in partial fulfillment of the requirements for the degree of

\author{
Doctor of Philosophy \\ in \\ Technology Management
}

Dissertation Committee:

Antonie J. Jetter, Chair

Eva Thanheiser

Charles M. Weber

Sung Yi

Portland State University

2020 
(C) 2020 Byung Sung Yoon 


\begin{abstract}
During concept development, product developers consider product users and their future experience, cost, development and manufacturing efficiency, product function/quality, and differentiation of the product in the market. Development teams often struggle to adequately address all of these considerations, due to the following reasons: (1) Differences in technological and experiential knowledge, methods used, and communication styles that make it difficult for customers/user, marketing, and engineering to communicate effectively. As a result, important factors may not be sufficiently considered. (2) Product design factors, including technological alternatives, functions, features, benefits, and customer value are interdependent: in some cases, customers are willing to sacrifice a feature for improvement in another factor, in other cases, they only value particular design factors if other factors are also present. Design factors can therefore only be understood in the context of other factors. However, current concept development methods fail to adequately model the system of concept development decisions. (3) The structural complexity of products hinders teams from assessing how a change impacts all other design factors and future customer value, which can cause teams to ignore indirect effects and unintended consequences of product concept decisions.
\end{abstract}

This research, therefore, presents novel method, cognitive distance reduction method (CDRM), that allows teams to systematically, holistically, and iteratively assess alternative product concepts and their respective impact on customer value by modeling them as combinations of product design factors. Teams can thus identify and select 
product concepts that achieve high customer value, given existing constraints. CDRM captures the mental models of engineers and marketing professionals about the elements and interdependencies of the development project (e.g., product features, benefits, customer value, and technologies) and represents them as quantitative system models to simulate future system states. CDRM consists of six steps (Basic PDF Elicitation, Model Formation, Model Synthesis, Scenario Building, Simulation, and Result Analysis \& Interpretation). CDRM is based on a system modeling approach, namely fuzzy cognitive mapping (FCM), that is gaining popularity in many fields but is still largely unused in product innovation. Two studies, both using robotic vacuum cleaners as the product concept of interest, are used to implement, test and assess the proposed CDRM: a pilot study, focused on feasibility and an experimental workshop, focused on the impact of CDRM on product development teams. Results show that CDRM is capable of representing a new product as a system, comprised of product design factors and relationships among them. Complexity is managed by creating the customer-focused Need Map and the engineering-focused Tech Map independently and integrating them to construct a group mental model, so-called PDF Map. The various maps capture the worldviews of PD team members and serve as a communication tool. Moreover, CDRM can also be used as a simulation tool and helps teams identify and select product concepts that achieve high customer value, given existing constraints. As part of the CDRM analysis and simulation, sensitivity analysis helps product development avoid overengineering or not meeting minimum requirements by identifying PDFs that do not contribute to further improvements of customer preference or might even have detrimental effects. 
The primary contribution of this research is practical by providing a novel approach for helping product development engineers capture and understand customer knowledge for successful concept development activities. CDRM can improve current concept development practice by improving engineers' understanding of customer requirements and select product concepts that best fulfill customer needs. To make these practical contributions, theoretical and methodological improvements were necessary. Regarding theory, the work provides a comprehensive discussion of several phenomena that plague early product development and knowledge sharing and provides clear differentiation between uncertainty, complexity, and equivocality, describes how they impact team mental models and provides an explanation of cognitive distance. The work integrates several current research perspectives. Regarding methodological innovations, this work provides several approaches for measuring cognitive distance based on survey data and Fuzzy Cognitive Maps that can be used by practitioners and researchers who wish to understand if teams interpret a complex system in a similar way or, instead, suffer from equivocality. 


\section{Acknowledgements}

It has taken a long time to reach this point. I spent countless days in my cave (my office at Portland State University) reading superb research articles in various fields and dreamed to bring this dissertation to the world. I never hesitated to learn new things to fulfill any missing gaps for my research, and my brain was full of endless ideas. However, most of them were in the clouds and not grabbed clearly in my hands. I needed someone to talk and discuss my foggy ideas about the research. Fortunately, on all such occasions, I had someone to share the ideas, and he/she helped me to make those clear and concrete. It is a great pleasure to express my gratitude and sincere thanks to all who encouraged and supported me in carrying out this study.

First and foremost, I would like to express my deepest appreciation to my advisor Dr. Antonie Jetter who was more than generous with her expertise and precious time. She always guided me in the right direction and help me in every situation all throughout my journey of Ph.D. I also have to say many thanks to the rest of my committee members, Dr. Charles Weber, Dr. Sung Yi, and Dr. Eva Thanheiser for their insightful comments and constructive suggestions on this study.

Without the tremendous help from my fellow colleagues and friends, I could not have completed this study. I wish to express my sincere gratitude to João Lavoie, Edwin Garces, Chih-Jen Yu, Pei Zhang, Ahmed Alibage, Amani Kaadoor and Rassaniya Lerdphayakkarat who were willing to give me comments and ideas through research group meetings. Also, I would like to say thanks to William Dresselhaus who provided 
invaluable advice. I cannot forget Seong Yun Cho with whom I discussed everything for this study with having lots of coffee in spite of different research fields.

Lastly, I have no words to describe how thankful I am for all of the sacrifices that my parents and all family members have made on my behalf. Especially, I cannot thank my wife, Joeun Park, enough for her indescribable love and devotion. 


\section{Table of Contents}

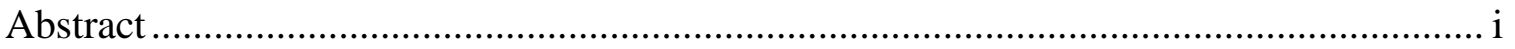

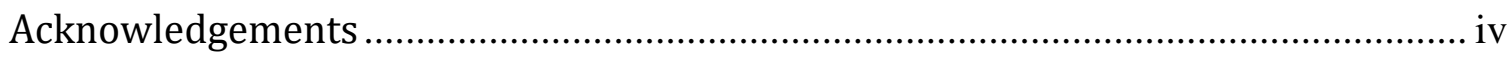

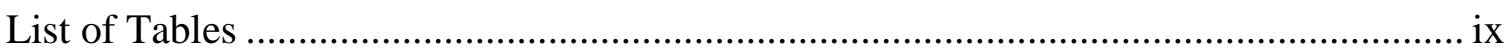

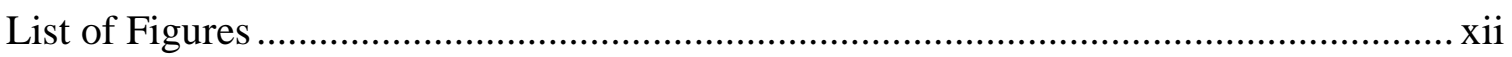

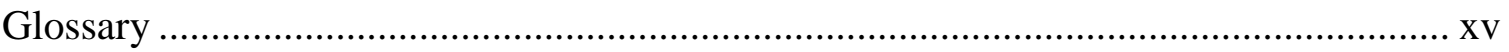

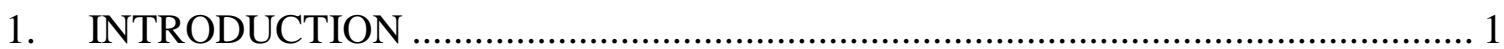

1.1. Research Background ................................................................... 1

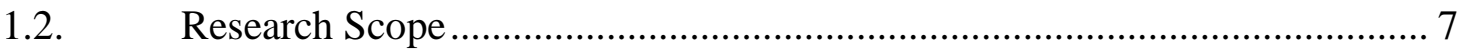

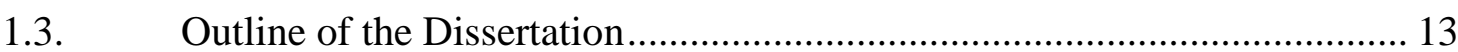

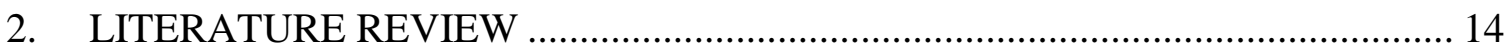

2.1. Cognitive Distance Reduction in PCD phase .......................................... 14

2.1.1. The Front End as the Context of Product Concept Development ............... 14

2.1.2. Equivocality, Uncertainty, and Complexity of Product Concept

Development in the Front End ..................................................................... 19

2.1.3. Barriers to the Effectiveness of Product Development Engineers............... 24

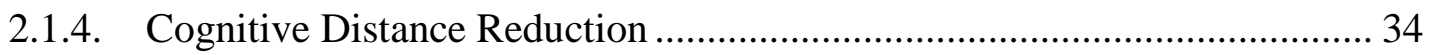

2.2. Product Design Factors as an Engineering Challenge ............................... 35

2.2.1. Product Design Factors in Product Concept Development ........................ 35

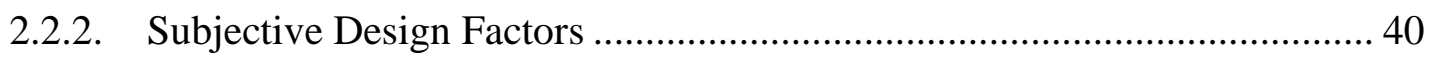

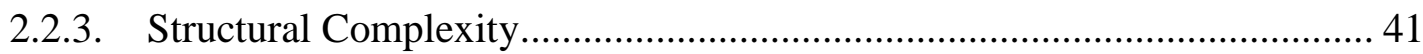

2.2.4. Summary: Challenges of Dealing with Product Design Factors ................ 42

2.3. The State-of-the-Art of Product Concept Development Methods.............. 43

2.4. Effectiveness of Existing Decomposition and Modeling Methods ............. 48

2.5. Requirements of a New Method ........................................................ 51

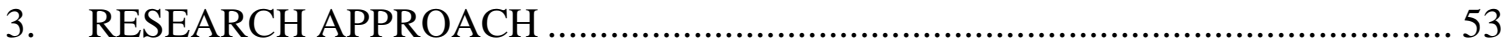

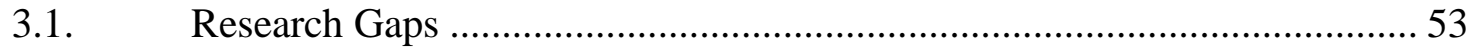

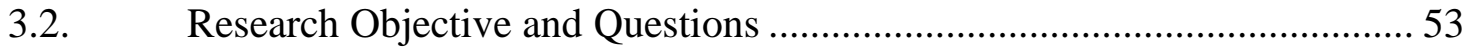

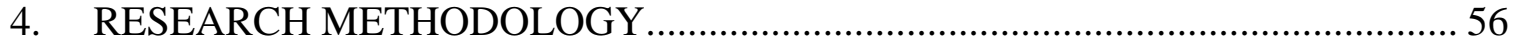

4.1. $\quad$ Background: Fuzzy Cognitive Mapping ................................................... 57

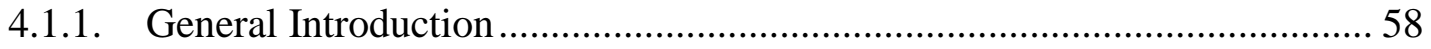

4.1.2. Aggregated FCM as a group mental model ............................................ 62

4.1.3. Fuzzy Cognitive Maps as a Core Part of the CDRM................................. 67 


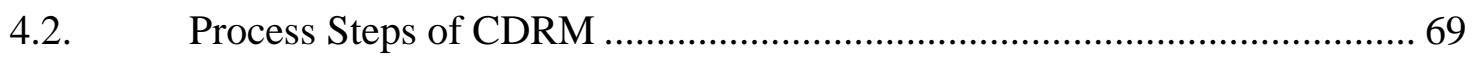

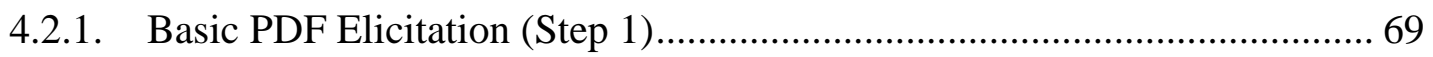

4.2.2. Model Formation (step 2) …………………......................................... 71

4.2.3. Model Synthesis (Step 3) ........................................................................ 74

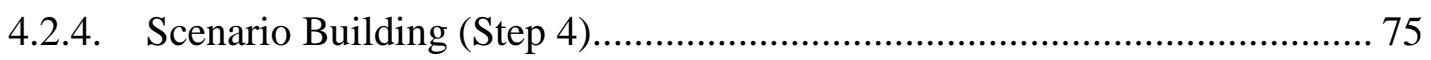

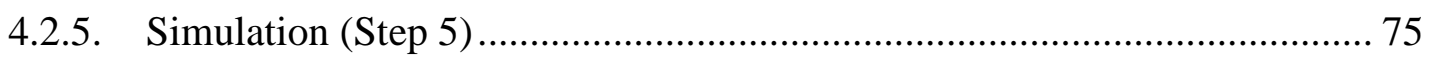

4.2.6. Result Analysis and Interpretation (Step 6)............................................... 76

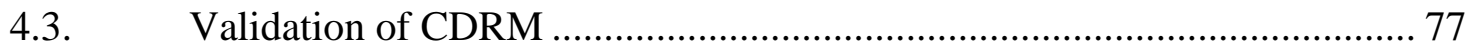

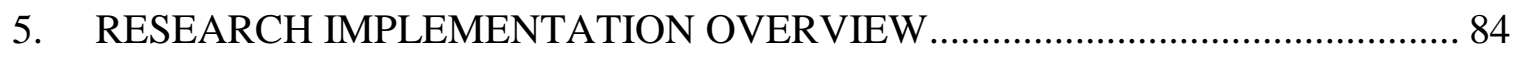

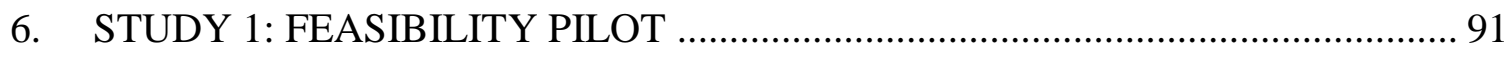

7. STUDY 2: DATA COLLECTION DURING A CDRM WORKSHOP ................. 102

7.1. Experiment Overview....................................................................... 102

7.2. Overview Study Setting and Participants of the Experiment .................... 104

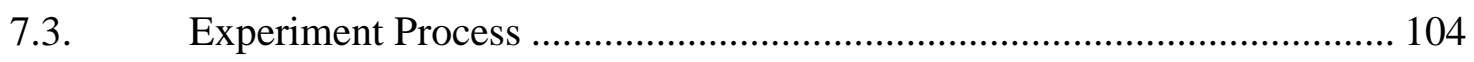

7.3.1. Product Information Collection .............................................................. 106

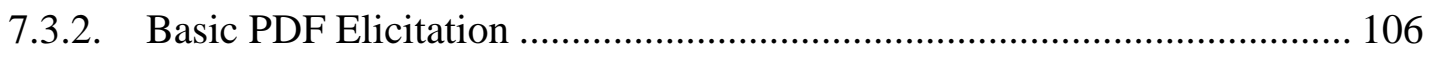

7.3.3. Need Map Development ........................................................................ 108

7.3.4. Participant Recruitment .......................................................................... 108

7.3.5. FCM Modeling/Concept Development Workshop..................................... 110

7.3.6. Observations on Workshop Dynamics ..................................................... 117

7.3.7. Map Aggregation and Simulation.......................................................... 118

7.3.8. Analysis \& Interpretation: ................................................................. 118

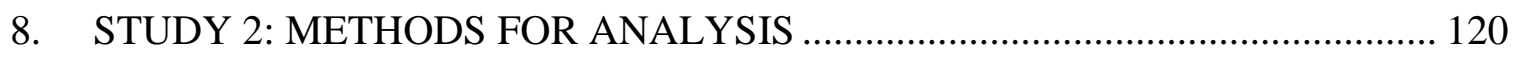

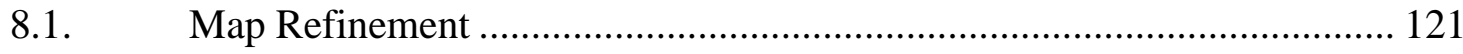

8.2. Consensus Measure ……………………......................................... 121

8.3. Map Comparison and Distance Ratio ................................................... 126

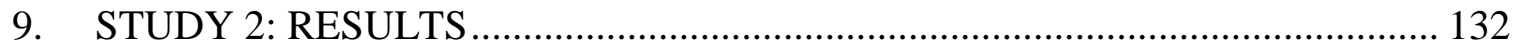

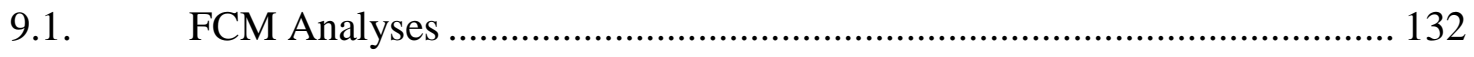

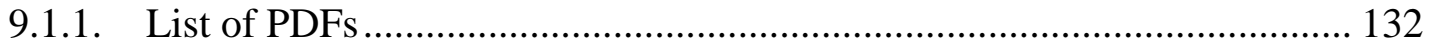

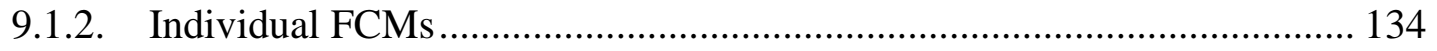

9.1.3. Group Maps - Tech and Need Maps ...................................................... 145

9.2. FCM Analyses of PDF Map and Simulation......................................... 152

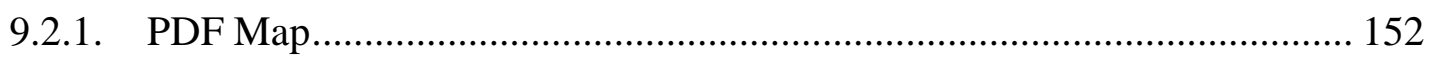

9.2.2. Scenarios - Product Concepts ...................................................................... 157 


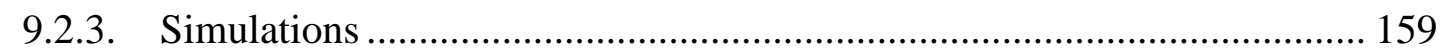

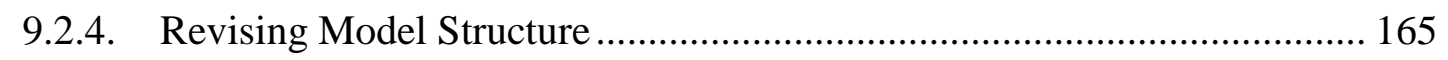

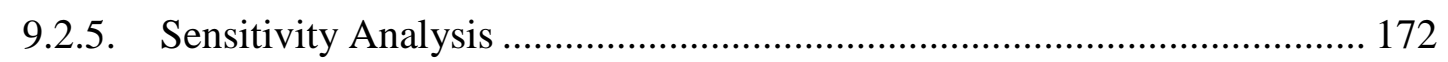

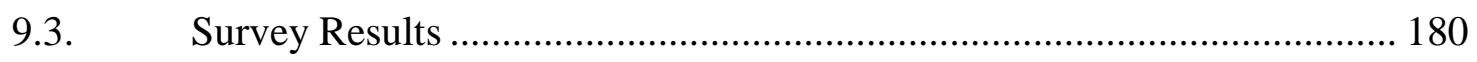

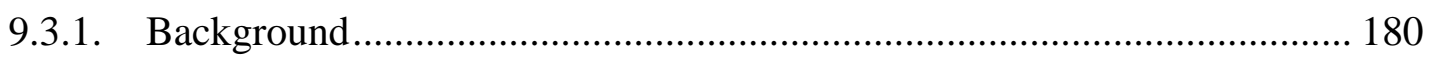

9.3.2. Change across surveys for individual participants ................................... 183

9.3.3. Change in cognitive distance before and after modeling ........................... 190

9.4. A Comparison between Simulation and Surveys ...................................... 196

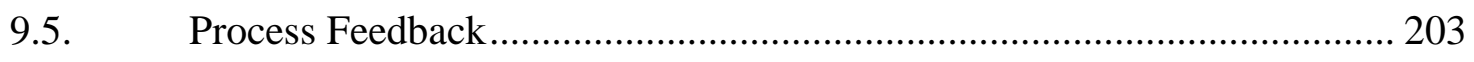

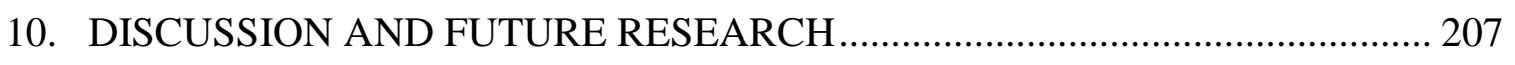

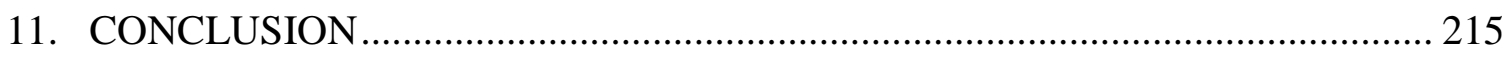

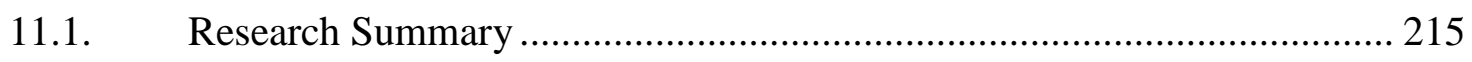

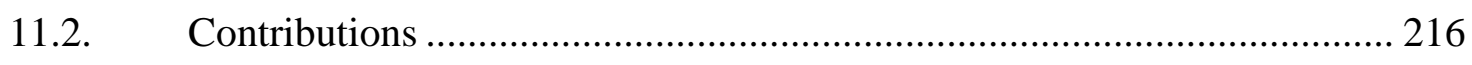

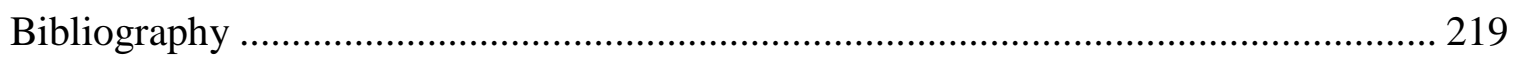

Appendix A - Axiomatic Design Approach (ADA) …………………….................... 236

Appendix B - Product Design Requirement Document ................................................ 239

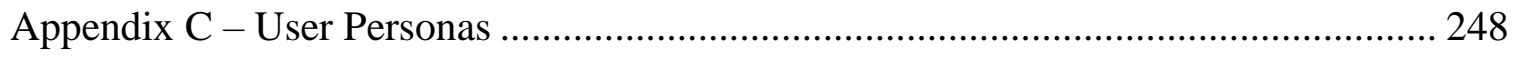

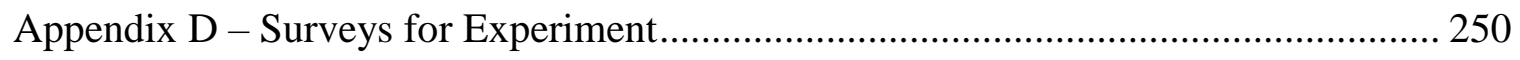

Appendix E - Recruiting Material ........................................................................ 269

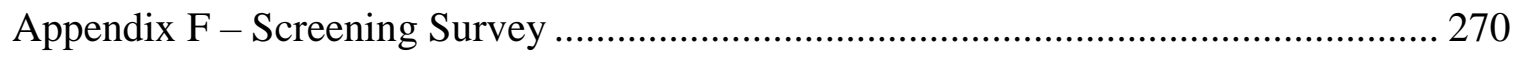

Appendix G - Workshop Handout ……………………....................................... 277

Appendix H - Survey Results............................................................................... 285

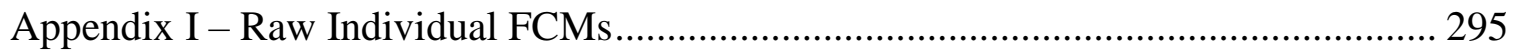

Appendix J - Refined Individual FCMs ...................................................................... 311 


\section{List of Tables}

Table 1.1 Comparison of traditional approach and user-centered design ..............................5

Table 2.1 Comparison of Functional and Cross-functional Teams....................................... 17

Table 2.2 Different orientation between R\&D and Marketing ..........................................2 20

Table 2.3 Customer Focus versus Managerial Focus............................................................20

Table 2.4 Characteristics of the constructs of uncertainty and equivocality ..........................23

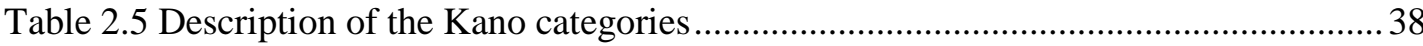

Table 2.6 Decomposition and Modeling in the product concept development phase.............47

Table 2.7 Evaluation of typical methods supporting product concept development .............51

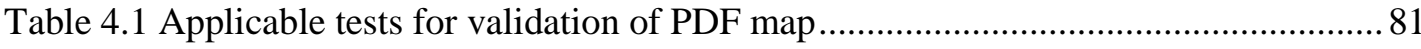

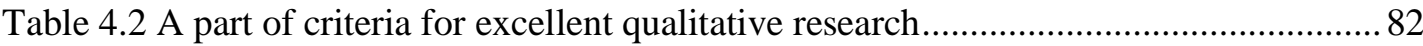

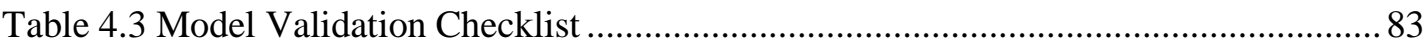

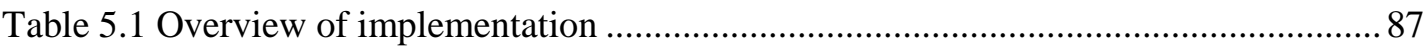

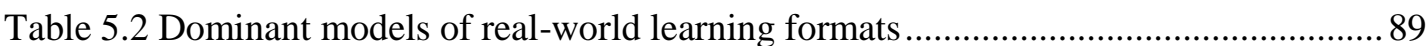

Table 5.3 Similarities and differences between NPD practices in education and in industry 90

Table 6.1 Definition of each concept in Tech Map................................................................93

Table 6.2 The adjacency matrix corresponding to Tech Map.............................................94

Table 6.3 Definition of each concept in Need Map ............................................................96

Table 6.4 The adjacency matrix corresponding to Need Map .............................................97

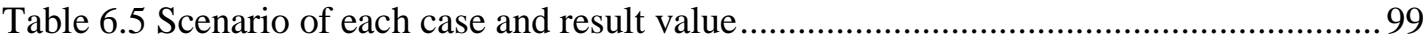

Table 7.1. Original PDF list elicited from product information ........................................ 106

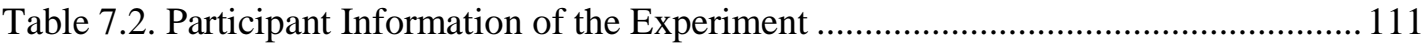

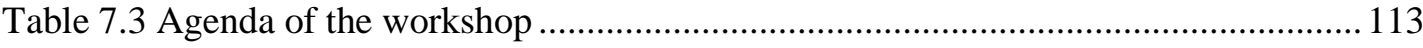

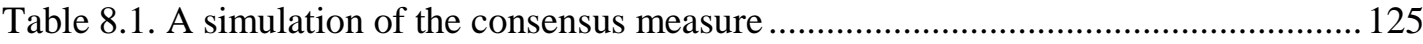

Table 8.2. Metrics for evaluating structural characteristics of an FCM.............................. 127

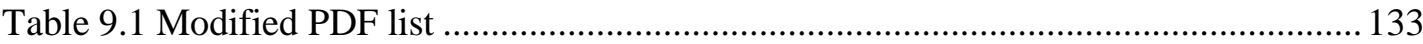


Table 9.2 Structural characteristics evaluation for the individual FCMs

Table 9.3 Distance ratio between E7's and E4's FCMs

Table 9.4 Relative comparison of distance ratios between individual FCMs 140

Table 9.5 Settled responses \& converged iteration number in each six FCM 143

Table 9.6 Settled responses \& converged iteration number in E7's and E8's FCM 144

Table 9.7 Adjacency matrix of Tech Map 147

Table 9.8 Adjacency matrix of Need Map. 148

Table 9.9 Structural analysis of Tech and Need Map 149

Table 9.10 Settled responses \& converged iteration number in Tech Map ... 152

Table 9.11 Adjacency matrix of PDF Map 154

Table 9.12 Structural analysis of PDF Map. 155

Table 9.13 Settled responses \& converged iteration number in PDF Map. 157

Table 9.14 Activation of product design factors for each scenario. 159

Table 9.15 Result values of the upper-level PDFs for Scenario 1 161

Table 9.16 Result values of the upper-level PDFs for Scenario 3 163

Table 9.17 Result values of the upper-level PDFs for Scenario 4 164

Table 9.18 Summary of simulation results 165

Table 9.19 Adjacency matrix of RPDF Map 168

Table 9.20 Structural analysis of RPDF Map .... 169

Table 9.21 Settled responses \& converged iteration number in PDF and RPDF Maps....... 171

Table 9.22 Summary of simulation results with RPDF Map 172

Table 9.23 Conversion of the Likert scale responses to ordinal values 183

Table 9.24 A part of the comparison results of central tendency and dispersion in the three surveys 185

Table 9.25 Comparison of the structural characteristics and the distance ratios between the raw and the new FCMs 194

Table 9.26 Simulation results for the impact of the eight target PDFs 197 
Table 10.1 Chapters addressing corresponding answers to each research question in research

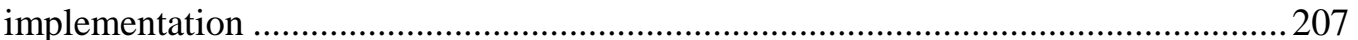




\section{List of Figures}

Figure 1.1 Cost commitment during product development .............................................. 1

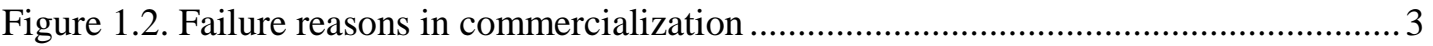

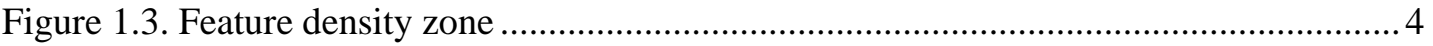

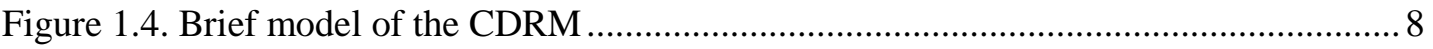

Figure 1.5. FCMs schematizing the causal relationships among product design factors......... 9

Figure 2.1 Equivocality, uncertainty and complexity ................................................... 19

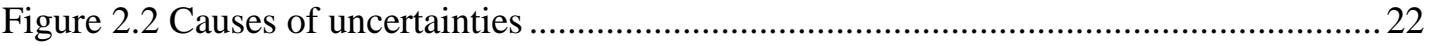

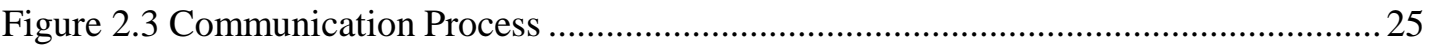

Figure 2.4 The path of information flow between engineers and customers ........................26

Figure 2.5 Conceptualization of a team mental model ......................................................29

Figure 2.6 The number of papers over top 10 research areas which includes the term

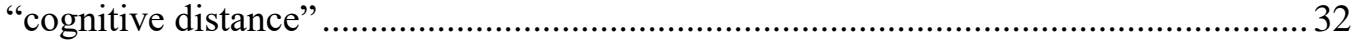

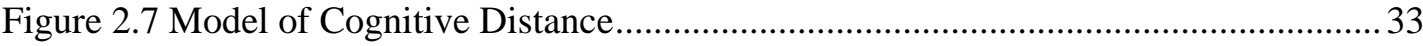

Figure 2.8 Conceptual model of cognitive distance reduction in PCD .................................. 34

Figure 2.9 The scope of product design factors corresponding typical definitions of attributes

Figure 2.10 Graphical representation of the Kano method .................................................. 39

Figure 2.11 Typologies of product design factors in the literature ...................................... 40

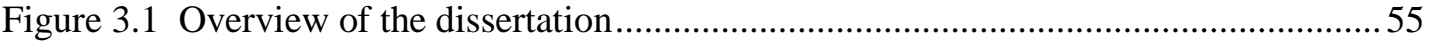

Figure 4.1 Five stages to develop and evaluate CDRM.....................................................56

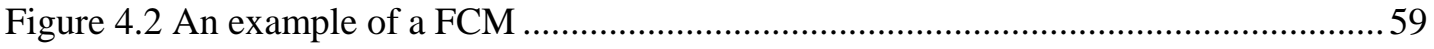

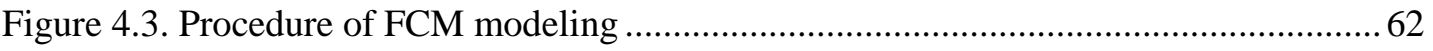

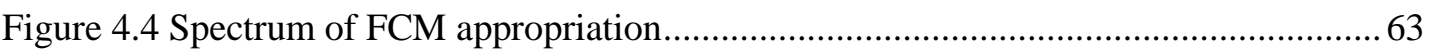

Figure 4.5 Procedure of a group FCM development by collecting individual FCMs............66

Figure 4.6 Product design factor map as the usage of FCM in CDRM ................................68

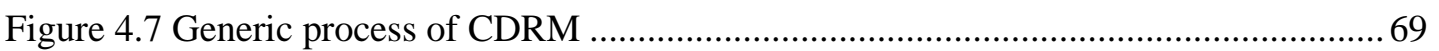




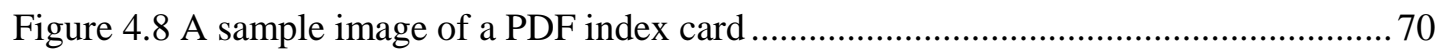

Figure 4.9 Integration of individual maps into a Tech Map ................................................ 73

Figure 6.1 Tech Map of a robotic vacuum cleaner design concept....................................92

Figure 6.2 Need Map of a robotic vacuum cleaner design concept ....................................95

Figure 6.3 Settled value of each goal concept ….............................................................. 99

Figure 7.1 Conceptual Model of the Experiment........................................................... 105

Figure 8.1 Expanded matrices for the calculation of similarity between two maps ............ 129

Figure 9.1 Degree of Centrality of each PDF in the eight individual FCMs ...................... 137

Figure 9.2 Degree of centrality of five main desired benefits in each participant's FCM... 138

Figure 9.3 Simulation responses to activation HEP..................................................... 142

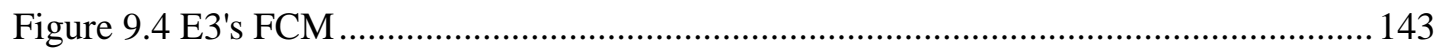

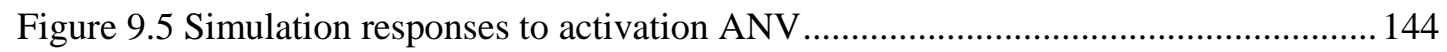

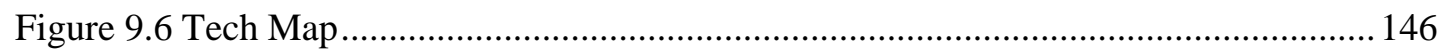

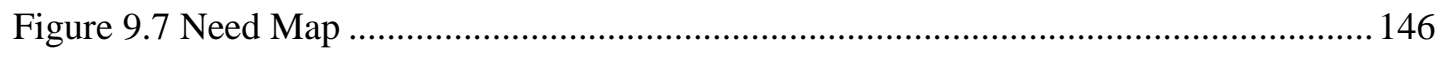

Figure 9.8 Centrality analysis of Tech (upper) and Need (bottom) Maps .......................... 150

Figure 9.9 Dynamic behavior of Tech and Need Maps ................................................... 151

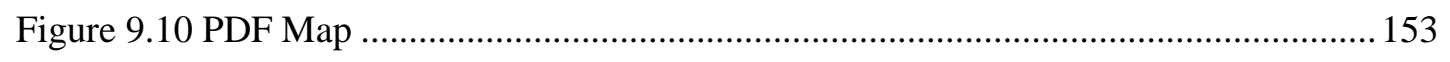

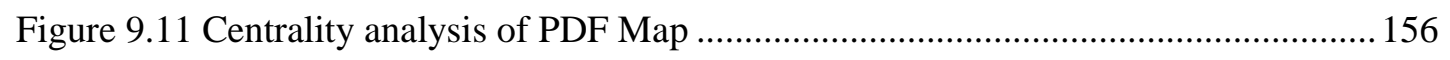

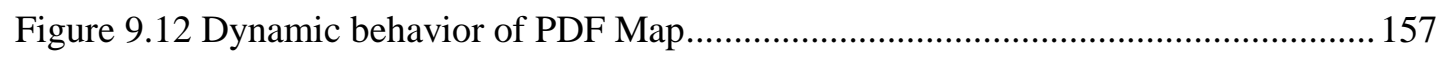

Figure 9.13 Dynamic behaviors of simulation results for Scenario 1 ................................. 161

Figure 9.14 Dynamic behaviors of simulation results for Scenario 2 ............................... 162

Figure 9.15 Dynamic behaviors of simulation results for Scenario 3 ................................ 163

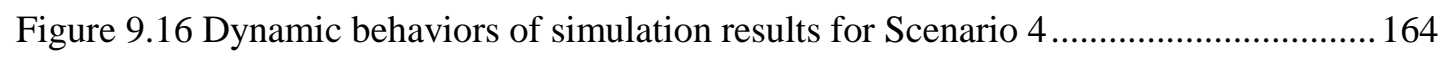

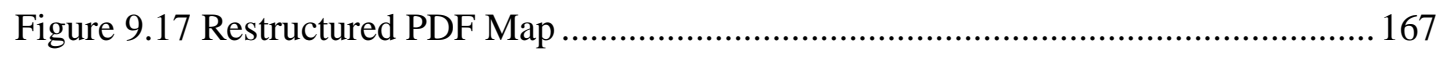

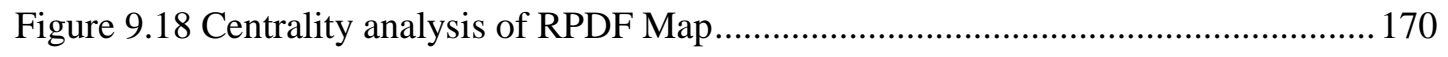

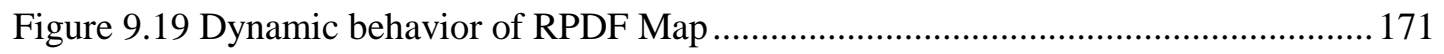


Figure 9.20 Sensitivity analysis for Scenario 1

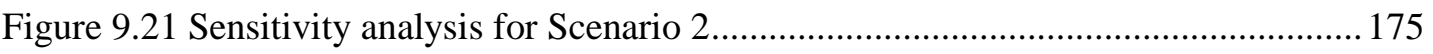

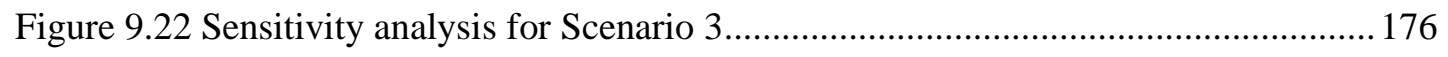

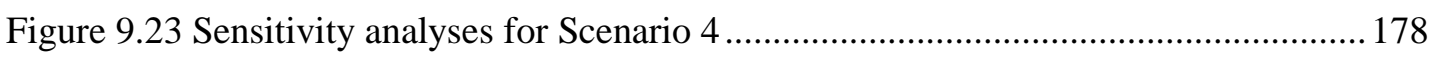

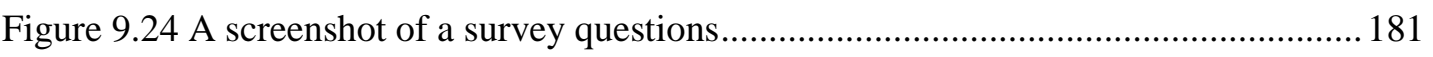

Figure 9.25 An example of response change a question during experiment........................ 184

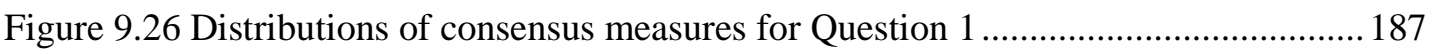

Figure 9.27 Distributions of consensus measures for Question 2 …................................. 188

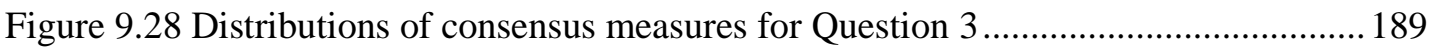

Figure 9.29 Comparison between the raw and the newly collected FCMs from E4 and E7 193

Figure 9.30 Distance ratio changes between Need Map and each individual FCM of

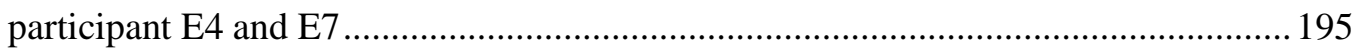

Figure 9.31 Comparison between simulation and surveys for Question \#1-1 ..................... 198

Figure 9.32 Comparison between simulation results and surveys for Question \#1-2 7.....200

Figure 9.33 Comparison between simulation and surveys for Question \#2........................ 201

Figure 9.34 Comparison between simulation and surveys for Question \#3......................... 202

Figure 9.35 Participants' self-estimation of improvement of their understanding as a result of individual FCM development activity .......................................................................20

Figure 9.36 Participants' evaluation of the help of individual FCM development activity in

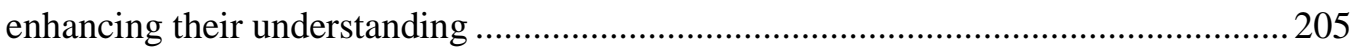

Figure 9.37 Participants' self-estimation of improvement of their understanding as a result of

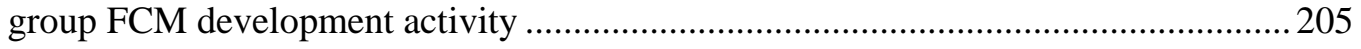




\section{Glossary}

Approach - a description of the overall mindset with which the research plan is to be conducted

Attributes - product design elements related to the characteristics of a product

Benefit - a way in which one or more features of the product provide a definable advantage, improvement, or satisfaction for customers

Cognitive Distance - differences in technological and experiential knowledge that causes different people to interpret, understand, and evaluate the world differently

Concept Development Stage - a phase of the generic product development process from product concept generation to product concept testing and selection

Customer Preference Value (CPV) - the value resulting from simulating product concepts as input scenarios in CDRM

Customer Value - a customer's perceived preference for and evaluation of those product attributes, attribute performances, and consequences arising from use that facilitate (or block) achieving the customer's goals and purposes in use situations

Decision Support - a way to help someone make important decisions

Equivocality - the ambiguity of communications or the existence of multiple and conflicting interpretations when sender (e.g., customers or customer-facing organizational units, such as marketing) and receiver (e.g., engineering) have different subjective views or frames of reference

Feature - a physical solution fulfilling a customer problem or a need

Framework - a set of ideas or facts that provide support for something

Function - something that the product must do or work to meet a customer need

Market-Oriented Stakeholders - the disciplines that convey customer views in FFE. They are marketing, sales or product management employees with regular or close contact with customers as well as customers themselves

Mental Model - a combination of the individual's subjective perceptions, concepts, ideas and perceived system status

Method - a systematic procedure, technique, or mode of inquiry employed by or proper to a particular discipline (e.g. participant observation) 
Methodology - a collection of problem-solving methods governed by a set of principles and a common philosophy for solving targeted problems

Model - a simplified framework designed to illustrate complex matters (e.g., concept, phenomenon, relationship, structure or system) graphically, mathematically, physically or verbally

Product Concept - a description of the form, function, and features of a product as a set of specifications, an analysis of competitive products, and an economic justification of the projects

Product Design Factors (PDFs) - sub-sets of product attributes that engineering teams can control, such as a product's form, function, and technical specifications. Non-technical product attributes that are outside of the control of engineering, such as brand image, advertising, packaging, and distribution, are excluded from the study unless they impact design factors.

Product Development (PD) - the transformation of a market opportunity and a set of assumptions about product technology into a product available for sale

Product Development Engineers - key professionals who are tasked with combining new and existing technologies into concepts that can satisfy customer needs, delight users, and fulfill project objectives (e.g., mission, resource, cost, and timing)

Technique - a procedure for accomplishing a desired outcome (e.g., interactional recording)

Technology - a capability given by the theoretical or practical application of knowledge, skills or artifacts

Technology Alternative - a technological element that can be selected in order to implement desired features or functions for a product

Tool - an instrument or certain tangible aid in performing a task or an operation (e.g., software)

Uncertainty - the difference between available information and the information needed to complete a task 


\section{INTRODUCTION}

\subsection{Research Background}

In the concept development stage $^{1}$ of new product development (NPD), which occurs in the so-called fuzzy front end (FFE) [1], [2], product development teams aim to develop strong product concepts that create superior customer value and are feasible within given technical, economic, and other constraints [3], [4]. The planning that occurs during the concept development stage sets the stage for project execution and determines how project risks are managed and how responsive the team can be to changes. Decisions made by the end of this phase determine seventy to eighty percent of total product cost [2], [5]-[7], as depicted in Figure 1.1

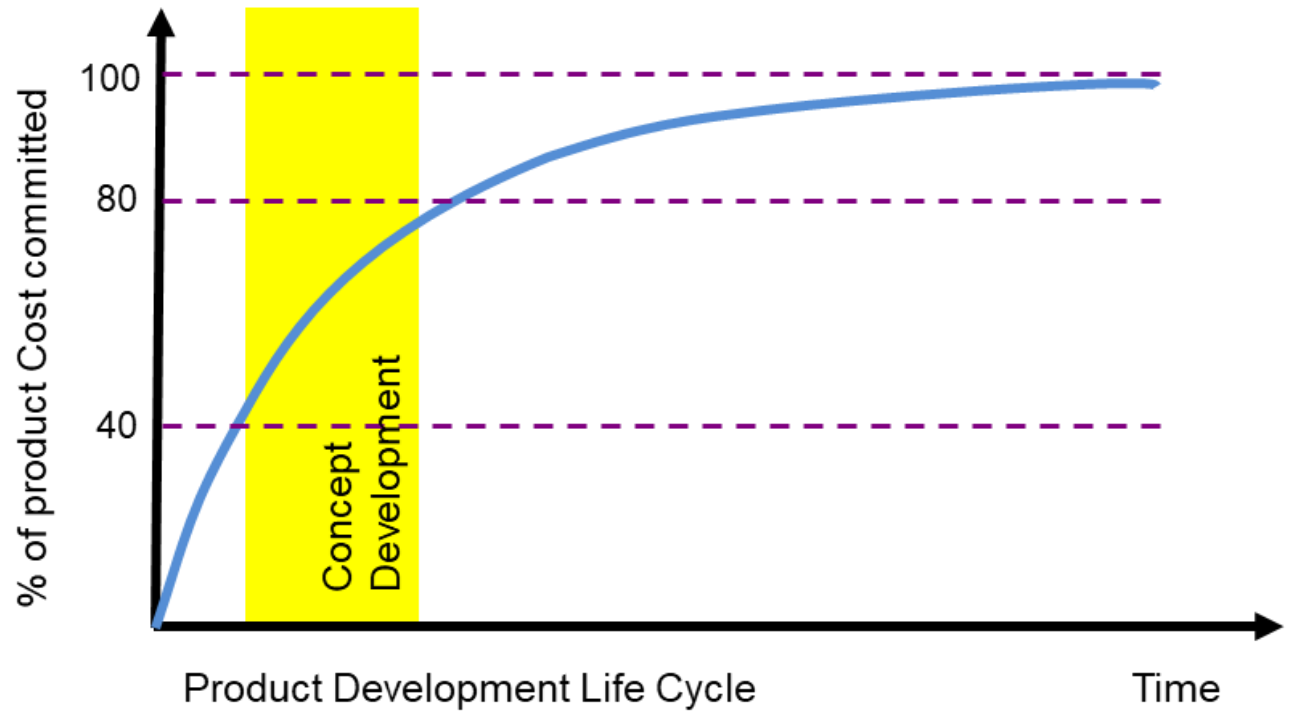

(Source: adapted from [7, p. 5])

Figure 1.1 Cost commitment during product development

\footnotetext{
${ }^{1} \mathrm{~A}$ phase of the generic product development process from product concept generation to product concept testing [3]. Herein, a product concept is defined as "a clearly written and possibly visual description of the new product idea that includes its primary features and consumer benefits, combined with a broad understanding of the technology needed" [125].
} 
Product development engineers have a key role in this process: they are tasked with combining new and existing technologies into concepts that can satisfy customer needs, delight users, and fulfill project objectives (e.g., mission, resource, cost, and timing) [8][10]. To this end, they carefully consider and select technological solutions that optimize product design factors ${ }^{2}$, such as desired features, functions, and benefits of a product. This occurs under high levels of uncertainty and frequently requires that engineers make tradeoff decisions between competing objectives and constraints [4], [5], [11]-[13].

Academic and practice-focused research in marketing, psychology, and engineering has long attempted to identify practices that lead to better combinations of product design factors [14]-[20]. Much of the work emphasizes the importance of supporting the work of development engineers. In particular, product development engineers should be capable of creating products with distinctive value to customers, based on a deep understanding of the value chain of product development [21], [22].

However, organizational and professional cultures, the division of labor between marketing and engineering, and work processes in NPD have traditionally incentivized product development engineers to show off technological excellence and innovativeness by integrating the latest cutting-edge technology into products, with only limited

\footnotetext{
${ }^{2}$ This study aims to enhance engineers' effectiveness in developing product concepts. The term design factors is therefore used to characterize a sub-set of product attributes that engineering teams can control, such as a product's form, function, and technical specifications. Non-technical product attributes that are outside of the control of engineering, such as brand image, advertising, packaging, and distribution are excluded from the study unless they themselves impact design factors. For example, the fact that a product comes with a five-year warranty (= product attribute) will not be considered in this study. However, if the long warranty provides a constraint on engineering decision making (e.g. the requirement to design a durable product), this constraint will be considered. Section 2 provides more detailed explanation.
} 
consideration for how this contributes to customer value ${ }^{3}$ [8], [23]-[26]. In doing so, they run the risk of developing and launching "feature-rich but experience poor" products [23], [24].

Figure 1.2 shows the results of a study on best practices in innovation management and summarizes typical reasons for failure to successfully commercialize products in the mechanical engineering industry [27]: The biggest culprit is a failure to meet market requirements by pursuing the technically perfect. The second most frequent cause of failure is a lack of differentiation of the product because it has too few attributes that set it apart from the competition. At the root of both failures is the inability to properly match market requirements with technical solutions in ways that not only satisfy but delight customers.

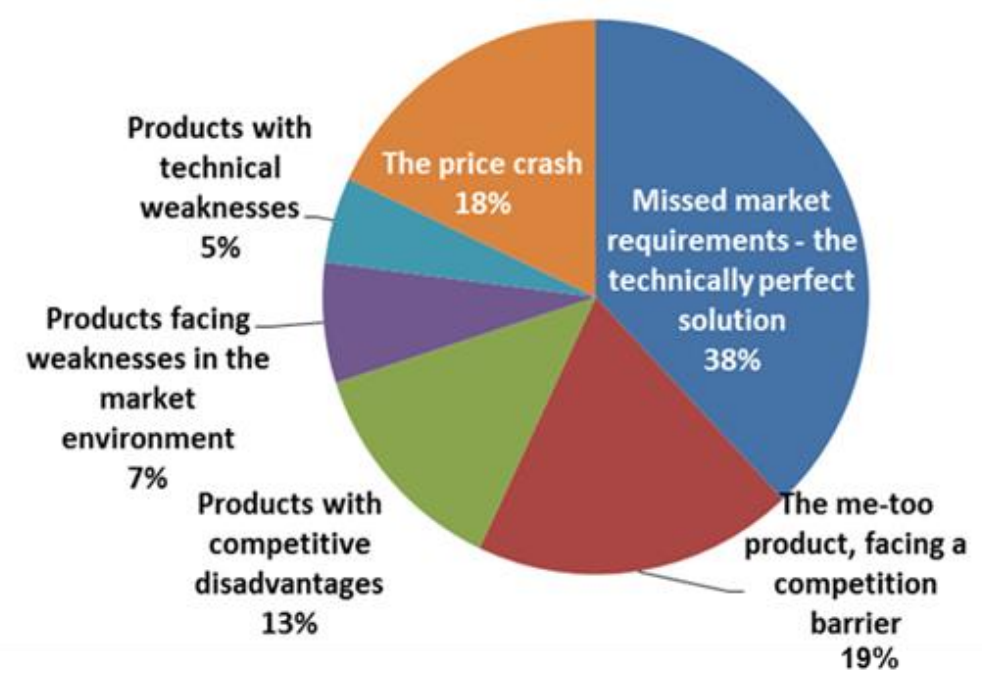

Figure 1.2. Reasons for Failure of Product Commercialization

\footnotetext{
${ }^{3}$ Woodruff defined this term as "a customer's perceived preference for and evaluation of those product attributes, attribute performances, and consequences arising from use that facilitate (or block) achieving the customer's goals and purposes in use situations" [32]. This study follows Woodruff's definition for this term.
} 
In this context, too few, poorly differentiated design factors can be equally detrimental as too many features that overshoot market needs, as Figure 1.3, which is taken from a study on the problems of over-specification and over-engineering, illustrates [28].

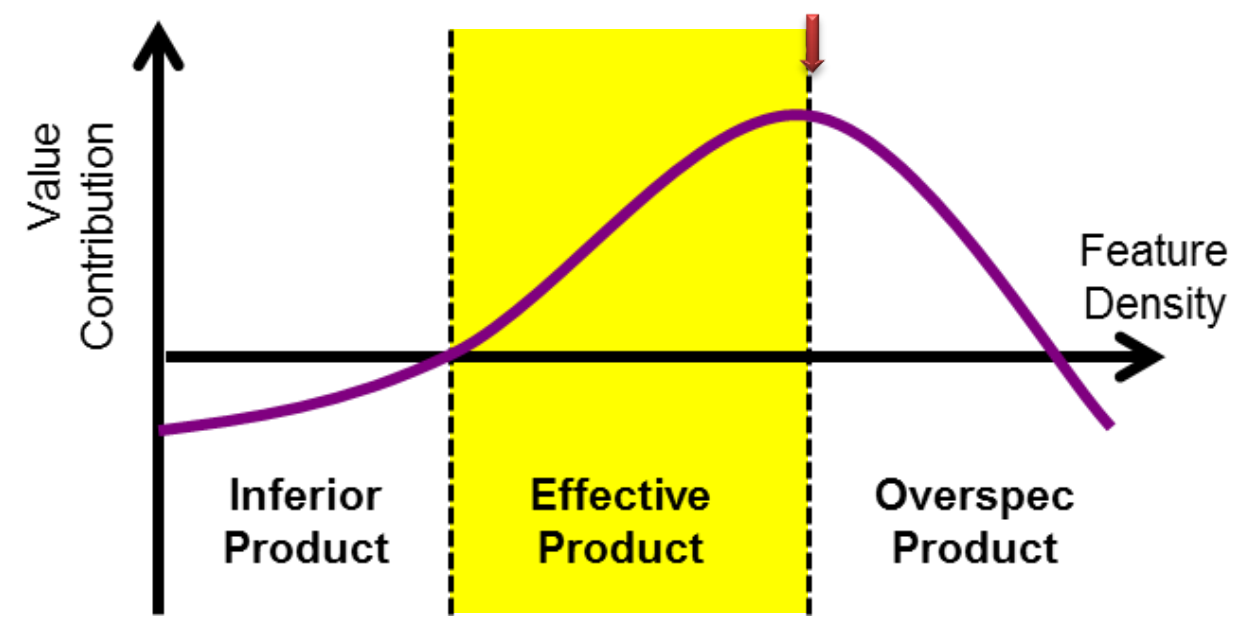

Figure 1.3. Feature density zone

Companies wish to create products in the effective range that are neither over- nor under-engineered to overcome the notoriously high failure rate of new products, which ranges from $35 \%$ to $45 \%$ [29].

To achieve this objective, research in the last two decades increasingly emphasizes user-centered innovation, rather than the traditional manufacturing-centered approach in NPD [23], [30]-[43]. The differences are outlined in Table 1.1 [37], [44]: User-centered design (UCD) focuses on the actual users of products and their experience in addition to cost, efficiency, quality and product variety which are traditionally believed to be primary sources of competitive advantage [45]. 
Table 1.1 Comparison of traditional approach and user-centered design

\begin{tabular}{ll}
\hline Traditional Approach & User-Centered Design \\
\hline Technology-driven & User-driven \\
Component focus & Solution focus \\
Limited multidisciplinary cooperation & Multidisciplinary team work \\
No specialization in user experience & Specialization in user experience \\
Some competitive focus & Focus on competition \\
Development prior to user validation & Develop only user validated designs \\
Product defect view of quality & User view of quality \\
Limited focus on user measurement & Prime focus on user measurement \\
Focus on current customers & Focus on current and future customers \\
\hline
\end{tabular}

(Source: adapted from [42, p132])

As a result of this new orientation, there have been important changes to stakeholders, process and organization in NPD [44], [46], [47]: Product development engineers are now required to access customer knowledge more closely and deeply than before [37], [44], [46], [48]-[52]. This also changes the nature of their interactions with other customer-facing disciplines, such as marketing, sales, and quality control personnel, who help development engineers gain deep customer insights, rather than staying exclusively focused on technology excellence [25], [36], [47], [53], [54]. The improved communication and deeper appreciation of the customers' problems are expected to improve the effectiveness of product development engineers in responding to the new challenges in NPD [15], [25], [26], [38], [53]-[62].

Specifically, these new "user-centric" practices aim to resolve equivocality - a situation in which sender (e.g., customers or customer-facing organizational units, such as marketing) and receiver (e.g., engineering) have different subjective views or frames of reference and therefore interpret the same information differently. When equivocality 
is resolved the cognitive distance ${ }^{4}$ between engineers and market-oriented stakeholders ${ }^{5}$ is reduced, which allows teams to communicate and coordinate their work more effectively and manage uncertainty and complexity. To achieve these objectives, several studies propose tools and methods that enable engineers to systematically connect their technology choices and engineering design decisions to market or customer needs [1][3], [11], [12], [15], [22], [63]-[77]. They include, among others, Pugh's evaluation matrix, quality function deployment (QFD), data envelopment analysis (DEA), and analytical hierarchy process (AHP).

However, to date, such tools and methods overlook two challenges with which NPD teams are often confronted during the product concept development phase [2], [3]: First, most current approaches fail to address the structural complexity of products: product design factors are frequently not independent of each other. Instead, causal relations exist among them and between them and customer value. In some cases, customers may only value particular design factors if other factors are also present. In other cases, they may be willing to accept trade-offs. Current approaches fail to consider these complexities. As a result, it is difficult for engineers to assess how a change in one factor impacts all other design factors and future customer value [25], [26]. Second, most current approaches only support sequential information flows: first, all market needs are identified, then targets for design factors are set, and then engineering decisions are made to meet these targets. However, some product design factors are so highly subjective that it is difficult to fully

\footnotetext{
${ }^{4}$ Differences in technological and experiential knowledge that causes different people to interpret, understand, and evaluate the world differently [151]-[154], [157].

${ }^{5}$ The disciplines who convey customer view in FFE. They are marketing, sales or product management employees with regular or close contact with customers as well as customers themselves [47].
} 
understand and anticipate their impact on the quality of product concepts upfront. Instead, this understanding has to be achieved iteratively through experimentation.

There consequently, is a need for new practical approaches that permit engineers to systematically, holistically, and iteratively assess how their proposed technical solutions (i.e., their choice of design factors) contribute to customer value so that they can select designs that are truly customer-, rather than technologyfocused.

\subsection{Research Scope}

This work addresses the needs identified above and responds to the increased demands on engineering, namely the need to contribute to user- and open innovation, to improve customer-orientation, and to improve the effectiveness of engineering decisions in product concept development. To achieve these goals, this research develops a novel method that reduces the cognitive distance and the resulting conflicts (or equivocality) between engineering and market-oriented stakeholders. Hereafter, this novel method is referred to as Cognitive Distance Reduction Method (CDRM).

CDRM is focused on the concept development phase, especially concept analysis and evaluation, of technology-driven products, during which customer requirements and information are translated into a specific combination of product design factors. Within this scope, the research focuses on technology products for the consumer market.

CDRM quantitatively models how product design factors have an impact on customer value to support product development engineers in creating and selecting 
product concepts that boost customer value and improve product success. Figure 1.4 depicts a brief model of the CDRM.

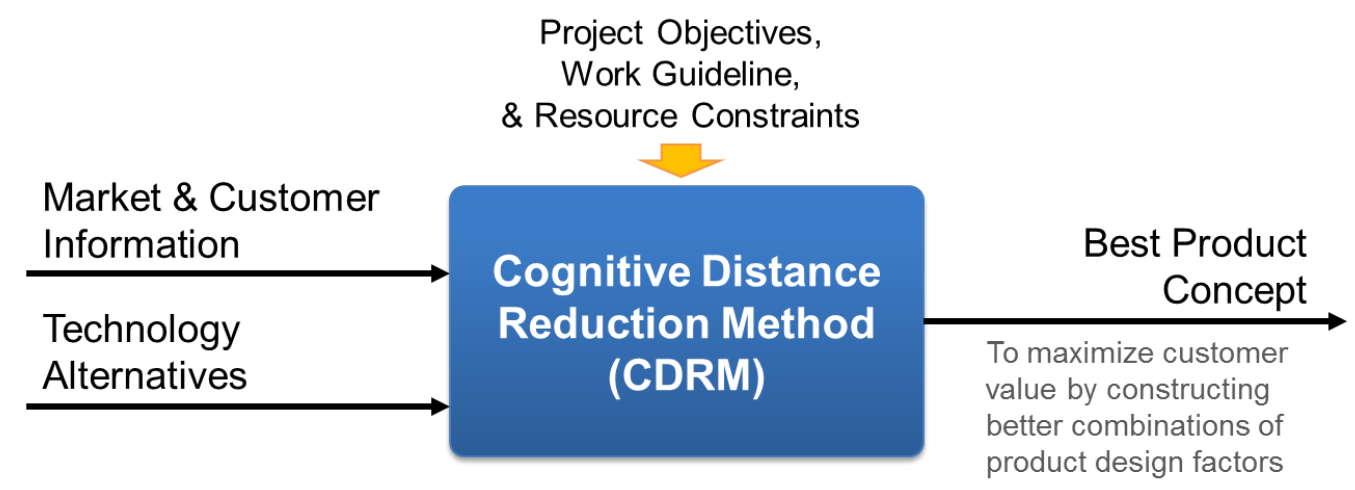

Figure 1.4. Brief model of the CDRM

The CDRM requires two inputs: market \& customer information, which is provided by market-oriented stakeholders (e.g., marketing, service, lead customers) and ideas for combinations of product design factors, as technology alternatives, from product design engineers. Product Project objectives, work guidelines, and resource constraints provide restrictions for the product development project [3]. The objective of CDRM is to help engineers assess their ideas for alternative product concepts by modeling them as combinations of product design factors and to determine each concept alternative's impact on product value, so that engineers can select good product concepts that achieve high customer value, given existing constraints. CDRM modeling uses fuzzy cognitive mapping $(\mathrm{FCM})$ as the core technique.

To capture engineers' or market-oriented stakeholders' perception of how product design factors impact each other and affect customer value, two cognitive models are developed: Market and customer information are modeled in a so-called Need Map, and design factors are modeled in a Tech Map. These two models are integrated into the 
product design factor map, which is the core model of CDRM. As depicted in Figure 1.5, the core model is comprised of five layers: technology alternatives, features, functions, benefits, and customer preference.
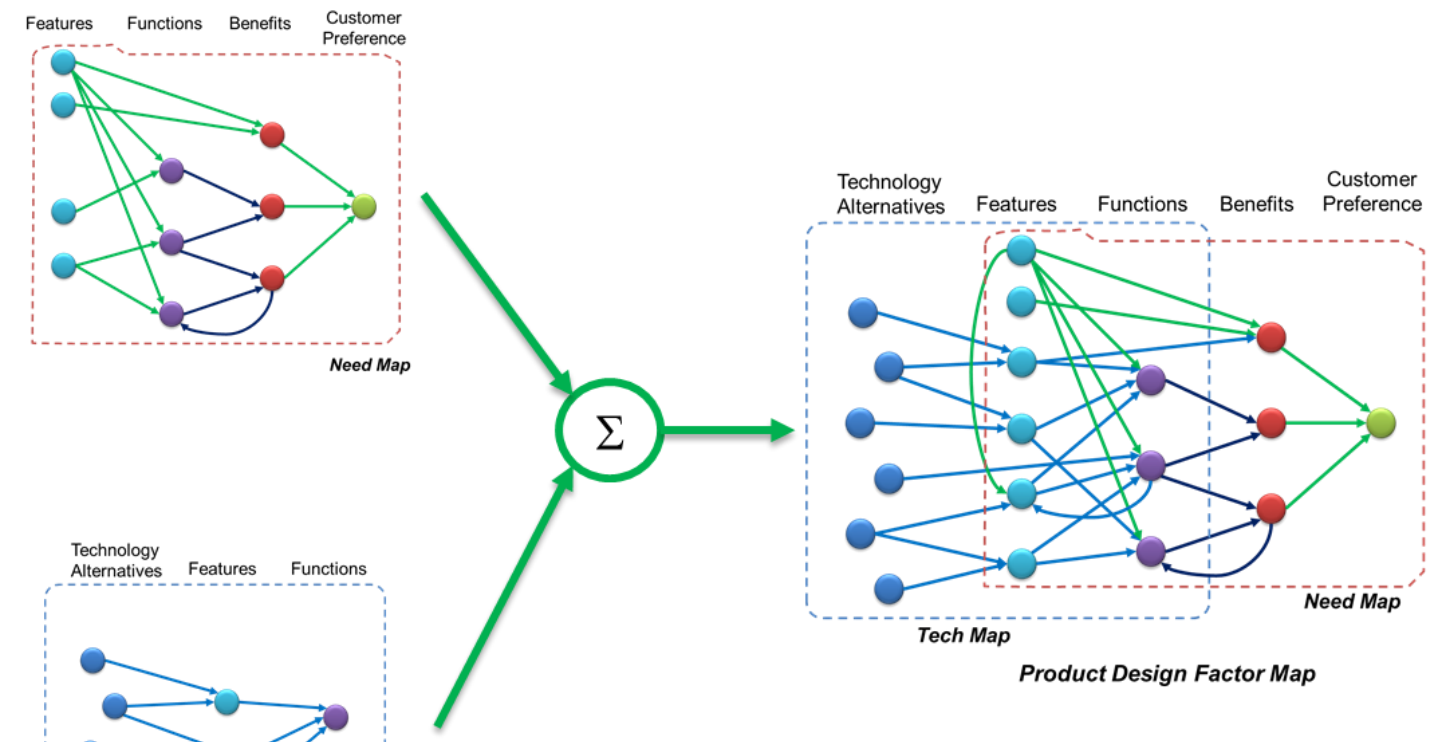

\section{Figure 1.5. FCMs schematizing the causal relationships among product design factors}

The model is used to simulate how different product concepts, represented as combinations of product design factors, impact customer preference, given the customer's needs and desires. Each product concept alternative ${ }^{6}$ is a unique scenario or "simulation run." For each scenario, the corresponding customer preference value (CFV) is calculated and compared against the value achieved by other concepts. Because of the nature of FCM computation, CFV fall in the range between -1 and 1 . Product concepts

\footnotetext{
${ }^{6}$ Only technologically and economically feasible concepts are considered
} 
with a higher positive value, near or at +1 , are preferable to concepts with lower positive values. Concepts with negative values do not achieve customer value. CDRM can thus be used to select the best product concept among existing alternatives and to assess how suggested changes to product concept alternatives impact customer value.

The primary contribution of this work is of practical nature as it results in a novel method that is excepted to improve communication within product development teams, provide a decision aid for creating and selecting product concepts, and help product development teams manage change. Specifically, the contributions are as follows:

Improved communication: The models underlying CDRM are jointly created by marketing and engineering members of the team, who share and visually represent causal relationships between product design factors. This helps them establish a common understanding of the product development challenge. Particularly, the method enables engineers to gain a deeper understanding of the relationship between product design factors from a customer perspective, including subjective and emotional factors, thus resolving equivocality.

Decision support: CDRM determines customer value for each product concept alternative that is being considered and thus helps product developers to select the alternative with the highest customer value. Moreover, by showing the comprehensive impacts of product design factors on value, it also supports the creative process of concept generation: product engineers can use the model to identify value drivers and come up with concepts that further improve them while reducing factors that would decrease customer value. 
Managing change: Product development projects are not stable -to react to changing customer needs and desires, as well as technological opportunities and constraints, engineering changes are common during product design, manufacturing and even after-market [78]-[80]. In some instances, these changes 'change the rules of the game': entirely new factors come into play or the links between factors may change. As opposed to other, more static methods, CDRM can deal with these changes easily by updating the underlying FCM models: FCM allows modelers to add or delete concepts and to modify weights and relationships without having to change other aspects of the model [81]. In other instances, changes may not require to modify the model structure but simply increase or decrease product factors as a result of engineering changes. CDRM allows product developers to assess how this change propagates to other product factors and ultimately impacts customer value, thus helping them to understand the impacts of their change decisions in a holistic manner.

\section{Application}

Given the expected benefits of CDRM, I consider the method to be suitable for product development projects with high equivocality (resulting from the difficulty of understanding and interpreting a multitude of product design factors, including subjective and vague factors), with high architectural complexity (which leads to many dependencies between product design factors), and with relatively short market and technology cycles (which lead to frequent changes). Accordingly, interconnected consumer electronic devices, such as smartphones, wearables, or tablet computers, are a potential application domain. In these markets, consumers have increasing and diverse 
demands. Product developers often react by integrating as many new features as possible to respond to diverse needs, to be technologically superior to competitor offerings, and to make preparations for future changes in customer needs. However, they often fail to consider how much customer value these designs actually deliver and run the risk of adding too many features [23], [24], [28], [82]. Coman and Ronen [28] report on a smartphone manufacturer that failed to launch its new ambitious phone, which was equipped with a game platform and multimedia console, on time because more and more capabilities were added in the early stages of the development. These frequent changes resulted in delays, and ultimately an unimpressive and overpriced product. Similarly, Don Norman, the former vice president of advanced technology at Apple from 1993 to 1998, said in the interview with Turner [83], "The hardest part of design, especially consumer electronics, is keeping features out." Mark Rolston, a former chief create officer at Frog Design, shares similar views and said, "Great products can be made more beautiful by omitting things." On the other hand, the beauty of simplicity may cause companies to disregard important features. Recently, Norman and Tognazzini criticized that Apple is pursuing the production of beautiful objects excessively rather than "providing the right functions, aiding understandability, and ensuring ease of use" [84]. By considering all interdependencies between product factors, including their indirect connections, CDRM can help identify those features that robustly drive value for customers, versus those that should be omitted.

Within the scope of a dissertation project, it is nearly impossible to iteratively develop, test and refine the CDRM in the context of a consumer electronics company: The concept development phase of a new platform product is highly confidential to 
protect plans from competitors. Also, projects can stretch out over months or years and often include multiple global locations. For the purpose of my dissertation, I, therefore, developed and tested the CDRM by utilizing NPD-related engineering projects in an educational setting.

\subsection{Outline of the Dissertation}

Including this chapter, the dissertation is organized into eight chapters. In the subsequent Chapter 2, a comprehensive literature review investigates existing methods for concept development with a focus on how the cognitive distance between engineers and other stakeholders, especially customers, is approached. Next, Chapter 3 presents the research gaps drawn from problems pointed out in Chapter 2, and research objectives and research questions are defined. Chapter 4 introduces an overview of the research methodology in this study. Chapter 5 presents an overview of the research implementation, and Chapter 6 demonstrates a feasibility pilot of the proposed method. Chapter 7 describes the data collection process through an experimental workshop. Then, Chapter 8 provides information on evaluations tools capable of demonstrating the effectiveness of CDRM. The data obtained from the experiment are analyzed thoroughly in Chapter 9. Chapter 10 discusses the feasibility of the CDRM, confirmation of whether the results of the experiment answer the research questions, and how CDRM could be improved for future usage in industrial practice. Finally, Chapter 11 outlines the conclusion and research contributions. 


\section{LITERATURE REVIEW}

The following chapter presents a comprehensive and in-depth literature review. Chapter 2.1 describes the challenges which product design engineers need to confront in the product concept development (PCD) phase and the phenomenon of "cognitive distance" among members of the product development (PD) teams. Chapter 2.2 reviews research on product design factors and the challenges they pose for development engineers. Chapter 2.3 reviews state-of-the-art methods for the concept development phase. Chapter 2.4 investigates the adequacy of these methods, and Chapter 2.5 identifies requirements of an improved, alternative method. These requirements will be used for the development of CDRM.

\subsection{Cognitive Distance Reduction in PCD phase}

This chapter describes the challenges PD engineers face in the PCD phase: it first describes the context of the PCD, which occurs in the so-called fuzzy front-end (FFE) and in cross-functional teams [9], [53], [85]-[87] with customer involvement [16], [30], [34], [88]-[91]. Next, it discusses uncertainty and equivocality as defining characteristics of the FFE. It then discusses barriers to the effectiveness of product development engineers in the context of PCD. Specifically, it discusses barriers in the communication process and barriers to building team mental models. Lastly, it introduces the concept of cognitive distance reduction as a means to overcome these barriers.

\subsubsection{The Front End as the Context of Product Concept Development}

PCD occurs in the FFE before a company decides to undertake a product development project: multiple concepts are created and reviewed, and good ideas from several concepts may be merged into a new concept. This process continues until a stable 
product concept definition is reached and the decision to move the project into product development is made. The PCD phase thus includes a range of activities from product concept generation to product concept testing [3].

According to Brentani and Reid [92], the FFE can be defined as the earliest stage of the NPD process. Similarly, Schoonmarker et al. define the FFE as a pre-development process between R\&D and the beginning of a formal product development process [93]. According to Stevens [94], the FFE is over when a go/no-go decision is made that determines if the project is abandoned or its formal NPD process begins, based on welldefined product concepts for subsequent development activities. This pre-development process consists of several subordinate activities, namely ideation, initial assessment, concept development, business case analysis, product definition and planning [94], [95]. They have no distinct boundaries between them and each activity is interrelated with others [96], [97], though phased project gates are recommended in order to align marketing and engineering activities through gate reviews [98].

Since Smith and Reinertsen [99] first introduced the term, the FFE has received growing attention by researchers and practitioners, who regard the FFE to be a crucial stage in the NPD process that strongly impacts product and project success factors [100], such as performance, cycle time or speed of the NPD process, and innovativeness [92], [101], [102]. For example, Verworn [103] reported that the early involvement of all departments during FFE influences project success by enhancing communication, and that well-defined product concepts and definitions in FFE enable PD teams to minimize product deviations during product execution. Based on this and similar findings, the FFE 
is considered to provide the greatest leverage for improving the entire innovation process [104]-[106].

However, firms tend to still devote limited effort to the FFE, unlike other NPD stages [96] and hesitate to input resources and investments for the FFE because of the high uncertainty and resulting risk of such investments [100], [107], [108]. Moreover, there is considerable debate about how to best manage the FFE. Khurana and Rosenthal advised that firms chose their approach to managing the FFE based on their size, decisionmaking style, operating culture and new product introduction frequency [96]. Given the interrelatedness of FFE activities, which leads to iterations, Koen et al. argued that management approaches relying on a sequential process are inappropriate for FFE management [109]. Instead, they proposed a new, "circular" model, the so-called new concept development (NCD) model that explicitly accounts for different pathways through the front-end, as well as for iterations [110]. Models similar to the NCD model have since been proposed by other researchers [95]. Also, particularly in software and information technology industry, agile product development is increasingly used to receive frequent and rapid customer feedback and more strongly involve the customer in product development [111]. Some of these agile development approaches, which are inherently iterative, are also used in other engineering disciplines [112]-[114].

In the FFE, activities occur in cross-functional, rather than functional, teams that consist of individuals from multiple functional disciplines such as engineering, marketing, manufacturing, finance and purchasing [115]. Table 2.1 shows the difference between functional and cross-functional teams [86], [116]. 
Table 2.1 Comparison of Functional and Cross-functional Teams

\section{Functional}

\begin{tabular}{|c|c|c|}
\hline Definition & $\begin{array}{l}\text { Activity within a } \\
\text { department that has } \\
\text { no contact with } \\
\text { personnel from } \\
\text { another department }\end{array}$ & $\begin{array}{l}\text { Activity that includes } \\
\text { personnel from different } \\
\text { departments who are asked } \\
\text { with ensuring assigned } \\
\text { objectives are achieved and } \\
\text { positions activity objectives } \\
\text { as superordinate to the } \\
\text { objectives of individual } \\
\text { functions and departments }\end{array}$ \\
\hline Dimension & $\begin{array}{l}\text { Cumulative knowledge } \\
\text { dynamics }\end{array}$ & $\begin{array}{l}\text { Combinatorial knowledge } \\
\text { dynamics }\end{array}$ \\
\hline \multicolumn{3}{|l|}{ Actors } \\
\hline $\begin{array}{l}\text { Heterogeneity in a } \\
\text { team }\end{array}$ & Low & High \\
\hline Cognitive distance & Low & High \\
\hline $\begin{array}{l}\text { Institutional } \\
\text { overlaps }\end{array}$ & High & Low \\
\hline \multicolumn{3}{|l|}{ Interaction Process } \\
\hline $\begin{array}{l}\text { Existing knowledge } \\
\text { base(s) }\end{array}$ & Broaden/deepen & Unification with others \\
\hline $\begin{array}{l}\text { Variety of contexts } \\
\text { to be integrated }\end{array}$ & Low & High \\
\hline $\begin{array}{l}\text { Investment in } \\
\text { mutual } \\
\text { understanding }\end{array}$ & Low & High \\
\hline $\begin{array}{l}\text { Bridging of } \\
\text { organizational, } \\
\text { technological and } \\
\text { sectoral interfaces }\end{array}$ & Low & High \\
\hline Rationale for Use & $\begin{array}{l}\text { Single domain } \\
\text { Decision speed crucial }\end{array}$ & $\begin{array}{l}\text { Multiple domains } \\
\text { Creative thinking } \\
\text { Problem-solving that pushes } \\
\text { the envelop }\end{array}$ \\
\hline $\begin{array}{l}\text { Knowledge Creation } \\
\text { Implication }\end{array}$ & $\begin{array}{l}\text { Low combination } \\
\text { Low externalization } \\
\text { Low internalization } \\
\text { Low socialization }\end{array}$ & $\begin{array}{l}\text { High combination } \\
\text { High externalization } \\
\text { High internalization } \\
\text { High socialization }\end{array}$ \\
\hline $\begin{array}{l}\text { Knowledge } \\
\text { management } \\
\text { Implication }\end{array}$ & $\begin{array}{l}\text { Organization data-to- } \\
\text { information }\end{array}$ & Organization knowledge \\
\hline
\end{tabular}

(Sources: combined with [86] and [116]) 
By sharing their domain expertise, members of cross-functional teams improve information flows and make sure that all important aspects of a product are considered early on. While this can lead to difficulties due to differences in world view, professional language and the way work is done [25], [26], [53], [57], [61], [62], [117], such teams can process customer inputs, understand internal and external restrictions, threats, and opportunities, and look at a whole set of stakeholders [36].

In addition to involving team members from different functions, many firms also involve customers in the FFE. One approach is the use of the "Lead User" technique, which was pioneered by von Hippel [34], [39], [118]. As a result of these trends towards user-centered innovation, PD engineers are expected to increase their attention to customers and future users of products. Within traditional engineering functions, PD engineers have to approach engineering work in new ways: they need to actively seek out user and market information and create technical flexibility to respond to new insights into customer needs. As members of cross-functional teams, they are required to broaden their roles beyond their technological expertise: they need to be able to share and incorporate knowledge across many fields, facilitate team learning, clarify project goals, and understand and deliver on customer expectations [36]. By combining deep technological expertise with cross-cutting skills (e.g., communication skills, skills related to work coordination, team skills, etc.), they become so-called "T-shaped" players [37]. 


\subsubsection{Equivocality, Uncertainty, and Complexity of Product Concept Development in the Front End}

The most frequently discussed characteristic of the FEE are uncertainty, complexity, and "equivocality. In a discussion of knowledge problems, Zack organizes these phenomena along a continuum based on the determinacy of the problem [119].

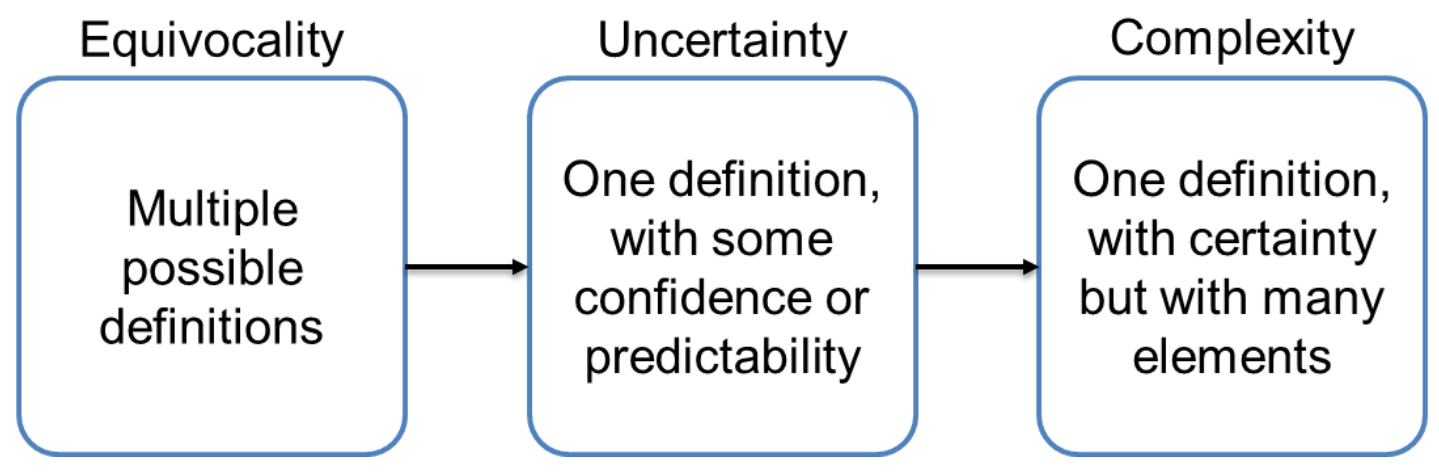

Figure 2.1 Equivocality, uncertainty, and complexity

Equivocality exists when team members have different subjective and conflicting interpretations of the issues at hand [108], [120]. Equivocality is regarded as the main cause of communication difficulties and conflicts within multidisciplinary NPD organizations [1], [108], [120]. In particular, a number of research have reported conflicts between marketing and R\&D/engineering [25], [26], [53], [55], [61], [62]. For instance, Shaw and Shaw [25] conclude that the main sources of conflict between engineers and marketers are poor communications and lack of understanding for each other's perspectives. While marketers focus on meeting customer preferences, engineers are concerned with the feasibility and effectiveness of technologies in NPD projects [57]. Accordingly, they look at issues from different vantage points and may interpret data differently. Table 2.2 summarizes different orientations between engineers in R\&D and marketing [31, p. 124]. 
Table 2.2 Different orientation between R\&D and Marketing

\begin{tabular}{lll} 
& \multicolumn{1}{c}{ R\&D } & \multicolumn{1}{c}{ Marketing } \\
\hline Time Orientation & Long & Short \\
Projects Preferred & Breakthrough & Incremental \\
Ambiguity Tolerance & Low & High \\
Department Structure & Informal & Moderately formal \\
Bureaucratic Orientation & Less & More \\
Orientation to others & Permissive & Permissive \\
Professional Loyalty & Profession & Firm \\
Professional Orientation & Science & Market \\
\hline
\end{tabular}

This subjective difference in knowledge, motivations, and experience also appears between PD engineers, who manage the product development process, and customers [121]. Table 2.3 shows a different focus on new products between customers and managers in high-technology industry.

Table 2.3 Customer Focus versus Managerial Focus

\begin{tabular}{l|l} 
Customer Focus & Managerial Focus \\
\hline Features & Design \\
Consequences, values & Cost \\
Ease of operation & Ease of production \\
Unique qualities & Unique technologies \\
Consumption & Production \\
\hline & (Source: adapted from [121])
\end{tabular}


Because equivocality is a result of individual differences, it will always persist at least at some level. If there is too much of it team members and outside stakeholders, such as customers, cannot combine their knowledge to reach a problem definition, and there is no agreement on goals, situations, and tasks. It is, therefore, necessary to unify the different visions either through negotiation, or by agreeing on a course of action that addresses and satisfies multiple meanings, or by leveraging hierarchical power to impose a single meaning that the team is to follow [119]. Too little equivocality, however, can hinder innovation because teams fail to explore alternative explanations and opportunities and shut down discussions too quickly.

Uncertainty is defined as " the difference between the amount of information required to perform a particular task, and the amount of information already possessed by the organization" [53, p. 224]. In contrast to equivocality, uncertainty exists in situations in which there is agreement on the task, and the team knows what it needs to know. However, there is a lack of information. According to Kim and Wilemon [100], uncertainties exist regarding technologies, markets, resources, and internal capabilities. Schröder and Jetter [73] categorized uncertainties of the FFE into four types: market uncertainty, technological uncertainty, environmental uncertainty, and uncertainty about resource allocation. Figure 2.2 shows the causes of each uncertainty. 
Market Uncertainty

- Change of the customer needs

- Future competitors

- Competitors' strategies

\section{Technological Uncertainty}

- Appearance of new technologies

- Timing of development
Environmental Uncertainty

- Change of social, economic, ecological and political environment

Uncertainty about resource allocation

- Lack of confidence for decisionmaking in each step caused by highly dynamic environment

\section{Figure 2.2 Causes of uncertainties}

These uncertainties can cause companies never to pursue an opportunity because it is considered to be too risky. They can furthermore lead to poor product concepts, wrong target markets, misallocations of resources throughout the development process, and, consequently, project failure. Thus, several researchers have suggested processes and methodologies for reducing uncertainties or managing their impacts. For example, Kim and Wilemon [122] recommend a holistic FFE process that is heavy on customer involvement. Their recommended approach to the FFE also nurtures multiple fuzzy ideas, rather than selecting single solutions too early and under high uncertainty [122]. Jetter et al. [47], [95], [123], [124] build on the idea that different levels of uncertainty require different approaches to the FFE and present a framework for selecting the FFE process according to the characteristics of an innovation project.

The effects of uncertainty and equivocality thus frequently occur together and impact the performance of the FFE activities [120]. Table 2.4 summarized the differences between uncertainty and equivocality concerning concept definition, key problem and response activities, and the consequences caused by the two factors in product development [120]. 
Table 2.4 Characteristics of the constructs of uncertainty and equivocality

\begin{tabular}{lll}
\hline & \multicolumn{1}{c}{ Uncertainty } & \multicolumn{1}{c}{ Equivocality } \\
\hline Concept Definition & $\begin{array}{l}\text { Difference between available } \\
\text { information and the } \\
\text { information needed to complete } \\
\text { a task }\end{array}$ & $\begin{array}{l}\text { The existence of multiple and } \\
\text { conflicting interpretations } \\
\text { among project participants }\end{array}$ \\
Key Problem(s) & Lack of information & $\begin{array}{l}\text { Lack of consensus and } \\
\text { understanding, and confusion }\end{array}$ \\
Response Activities & $\begin{array}{l}\text { Information acquisition and } \\
\text { analysis }\end{array}$ & $\begin{array}{l}\text { Exchanging of subjective } \\
\text { interpretations, consensus } \\
\text { formation, and enactment of } \\
\text { shared understanding }\end{array}$ \\
$\begin{array}{l}\text { Consequences (if not } \\
\text { sufficiently reduced) }\end{array}$ & $\begin{array}{l}\text { Increases risk in development: } \\
\text {-Difficulties in creating explicit and stable concepts, time } \\
\text { delays, waste of resources, difficulties in performing feasibility } \\
\text { analysis and project planning, concept failure }\end{array}$ \\
\hline
\end{tabular}

(source: adapted from [120, p. 552])

Both uncertainty and equivocality pose challenges for product concept definition. A product concept is defined as "a clearly written and possibly visual description of the new product idea that includes its primary features and consumer benefits, combined with a broad understanding of the technology needed" [125]. Uncertainty negatively affects this objective because PD teams may not know enough about upcoming technology changes, evolving user needs, possible competitor actions, and future organizational resources and priorities to develop solutions [94], [120]. Consequently, PD teams are required to put more effort into seeking additional information and may undergo trialand-error searches during the PCD, resulting in delaying time-to-market or missing the market window [94]. Equivocality has negative impacts on product concept definitions because it results in a lack of understanding [126], which makes it difficult for PD teams to process information provided by other team members or customers, and to come to a 
consensus on what product concept to select. As result, teams have multiple interpretations and experience confusion and conflict [120].

Moreover, they may choose product concepts based on incorrect assumptions, which may lead to concept failure [94]. To overcome uncertainty, researchers and practitioners have recommended cross-functional teams [127], [128], early involvement of critical functions [126], and strong customer involvement [129]. To overcome the equivocality that often occurs in these settings, they recommend early, frequent face-toface meetings, early resolution of conflicts between R\&D and marketing [53], and the use of integrators who are in charge of delivering agreement among various stakeholders [120], [130].

The challenges of equivocality and uncertainty are further increased through complexity: even if the PD team agrees on the task, and obtains relevant information to overcome uncertainty regarding said task, there are too many interrelated factors to consider and coordinate simultaneously [119]. Complexity is discussed in greater detail in section 2.2.3.

\subsubsection{Barriers to the Effectiveness of Product Development Engineers}

Equivocality, uncertainty, and complexity provide barriers to the effectiveness of product development teams because they result in communication barriers and limit learning. Both problems are discussed in the following.

\subsubsection{Barriers to Knowledge Exchange}

A message from one person (a sender) reaches another person (a receiver) through communication. The process is commonly conceptualized in a model that consists of six 
elements; source, encoder, message, channel, decoder, and receiver [131]. During the communication process, the original meaning of the message may be distorted due to internal and external factors, whereupon the receiver may interpret the message differently than the sender. Internal factors of distortion, such as communication skills, social attributes, knowledge level, and position within the social-cultural system, affect the sender when expressing an idea in language. They also affect the receiver in understanding the language sent by the sender [132]-[134]. Also, external distortion factors, such as language difference, illegible print, and background noise, may obstruct communication between the sender and the receiver [135]. These internal and external distortions limit the effectiveness of information transfer. Figure 2.3 depicts this: the lightning bolt on each arrow mark indicates disturbance at each communication process step.

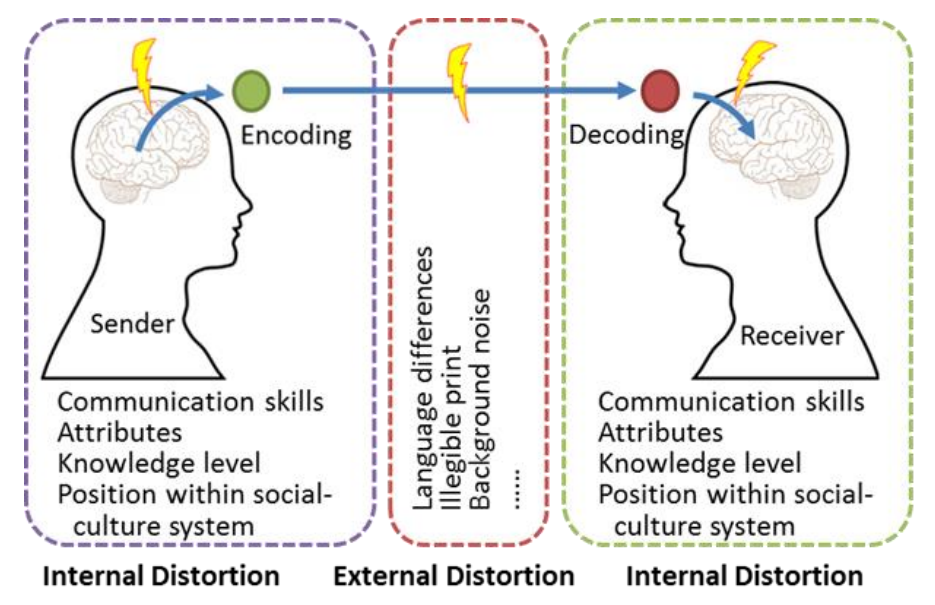

Figure 2.3 Communication Process

Human communication does not only consist of sending and receiving information but interpret information while doing so. "Encoding" and "Decoding" therefore involves complex cognitive processes: in their minds, people internally represent (or "map") the topic of interest in their brains, using a repertoire of thought processes. As will be 
discussed below, new information can be categorized within existing ways of thinking or may cause recipients to rethink their interpretations and representations [136]. Both cases constitute instances of learning.

This basic communication process does not only apply between individuals, but also between groups and other organizations. Internal distortions factors on the organizational level are caused by knowledge absorptive capacity, communicative capacity, and learning (by interaction) of each group or organization [136]-[138]. In the context of product development, the abovementioned distortions can limit effective communication and understanding between engineers, marketing, and customers, resulting in differences in how sensemaking occurs. The result is increased equivocality and a lack of commonly shared understanding.

Figure 2.4 depicts communication paths and knowledge flows between engineers and customers.

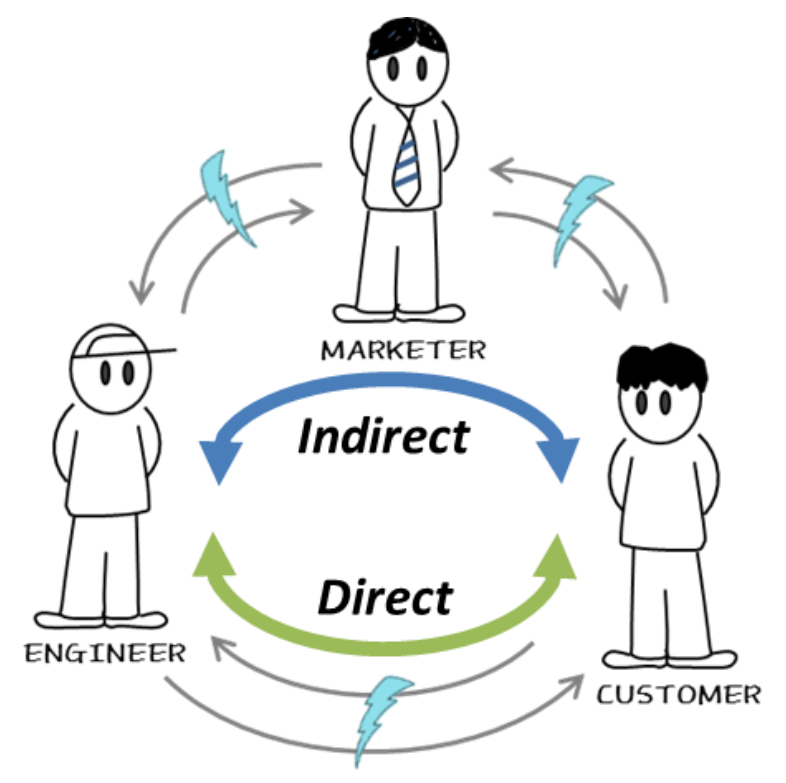

Figure 2.4 Paths of information flow between engineers and customers 
Disturbances of the information flow are indicated through lightning bolts: they can occur whenever the customer interacts with members of the product development team. Traditionally, customers interact with marketing, but, increasingly, engineers also interact with customers directly. When marketers act as intermediaries between engineers and customers, they can help the team facilitate successful information transfer. However, the interpretation of marketers can also negatively affect the quality of communication: marketers generally have backgrounds and experiences that are different from engineers [15], as well as different orientations when doing their work. As a result of these differences, customer insights may get 'lost in translation' when marketing communicates them to engineering. PD engineers may respond by developing insufficient technological specifications and selecting inappropriate technologies that do not fully meet customer needs.

Moreover, as already discussed, PD engineers are increasingly required to interpret customer inputs themselves and translate them into technological language, such as quantified product requirements or technology characteristics [139]. In industries that follow agile product development, PD engineers contact customers directly without intermediaries. This can lead to ineffective communication because customers' language is much different from PD engineers' one [140].

\subsubsection{Barriers to Learning}

Learning occurs when individuals or teams integrate experiential insights and knowledge from external sources into their mental models [141], [142]. Jones et al. [143] define a mental model as "a simple representation of reality that allows people to interact with the world." They argued that it is not a complete and accurate but temporary and 
functional representation of reality because of individuals' cognitive limitations. The mental model is continuously modified through learning. Additionally, mental models function as filters in processing incoming knowledge/information and can thus provide and impediment to learning.

Because of having distinctive capabilities, experiences, and socio-cultural backgrounds, different individuals have different mental models. When people interact with each other, their different mental models can lead them to interpret given knowledge/information differently. When confronted with knowledge that contradicts their mental model, team members may (1) accept new interpretations and modify their mental model, (2) reject the argument and adhere to their own mental model, or (3) ignore or suppress the conflicting information to avoid conflict and ruining relationships with other team members. Based on earlier work by Argyris and Schön, the first case constitutes so-called "double-loop learning": decision-makers gain insights into their own ways of seeing the problem and revise them, which enables them to plan new actions [141]. (Simply integrating the new knowledge into the already existing mental model would be a case of single-loop learning). The second and third cases are missed learning opportunities in that the mental model is neither put to use (by questioning and deepening it through new information that is fitted into it) nor modified (by changing it in response to new information). As the fourth option, team members may also engage in constructive conflicts that stimulate further discussion and co-construction (or collective construction) of a shared mental model. Such shared mental models benefit team effectiveness [144], [145]. 
Mohammed and Dumville [145] provide a similar perspective: according to them, team mental models refer to shared and organized understandings or representations of knowledge/information about equipment, working relationships, and situations associated with teams' tasks. The process of developing a team mental model from team member's individuals mental model requires team self-correction, which occurs through error identification, problem-solving, and feedback and results in corrected team attitudes, behaviors, and cognitions [145], [146]. Therefore, team mental model development is beneficial for team effectiveness because it reduces equivocality and reduces large differences between members' cognition of the team's task environment.

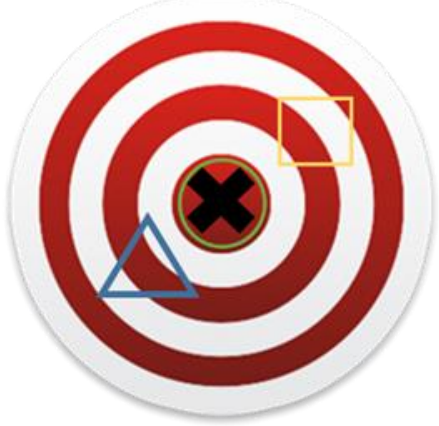

Case 1

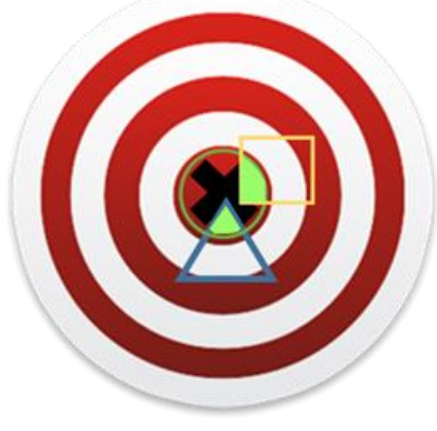

Case 2

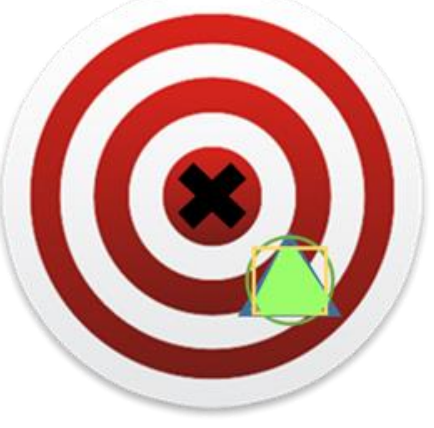

Case 3

Figure 2.5 Conceptualization of a team mental model

Figure 2.5 depicts the conceptualization of a team mental model as bright green shapes. The bullseye, marked with $\mathrm{X}$ represents the problem framing that is appropriate for a successful product development project. Circle, triangle, and square represent different team members' individual problem framings or mental models. Each member has different disciplinary knowledge (e.g. different engineering and marketing disciplines) and all three are required for a successful project. In Case 1, equivocality is very high and 
the team members interpret project goals, needs, data, processes, etc. differently. Accordingly, they cannot integrate their contributions and the project fails, even though all relevant knowledge is present and the green mental model is well aligned with the project. Case 3 is a case of very low equivocality - the team members essentially have the same mental model of the project but it differs substantially from actual problem needs. Accordingly, this project would also fail. Case 2 is a case of medium equivocality and partial alignment of mental models. Even though mental models are different there is sufficient overlap in the bullseye for the project to be successful. In this case, the green team member has an important role because she has not only appropriate problem framing and expertise in the green discipline but also sufficient overlap with the other team members to act as an integrator. Team members with this kind of experience are sometimes referred to as "T-shaped professionals" [48] because they have deep expertise in one field (the stem of the T) and a large, well developed horizontal axis that helps them span across other disciplines and mental models.

It should be noted that the location of the bullseye, i.e. the appropriate problem framing, is not known during the project because of the uncertainty of product development discussed above. Low equivocality with tightly overlapping mental models cover only a small area of the target and are therefore likely to be positioned outside of the narrow bullseye. This explains while diverse teams tend to perform better in innovation, as discussed in the next section.

As mentioned above, a team mental model consists of multiple individual mental models represented as shapes in Figure 2.5. The intersection of shapes stands for the 
shared (embodied) mental model of the team, while the area that has no overlap with other ones is regarded as complementary mental models ${ }^{7}$ [147].

In summary, there is a complex relationship between mental models and learning: on the one hand, mental models act as a lens through which information, including information about the mental models of other team members, is assessed. As such, mental models can impede learning. On the other hand, mental models can be changed through learning. Moreover, if a team learns in ways that increase the overlap between individual mental models and develops a shared understanding of the project - a so-called team mental model - it increases its effectiveness. In all of these processes, the difference between the initial mental models matters: models that are too different or too alike minimize learning. Accordingly, it is important to operationalize the differences/similarities of mental models, which is also known as "cognitive distance."

\subsubsection{Cognitive Distance - A Measure of Difference between Mental Models}

The term "cognitive distance" has been used in various academic fields. The bar chart in Figure 2.6 shows the top ten research areas that use the term, as well as the frequency of use. (The chart reflects the results of a keyword search for "cognitive distance" in Web of Science $\left.{ }^{\circledR}\right)$. Depending on the field of study, definitions of cognitive distance differ: For example, studies in environmental science-related articles define cognitive distance as the difference between people's perceived distance and their actual physical or spatial one [148]-[150]. Whereas, in management-related studies, cognitive distance refers to differences in perceived and experiential knowledge that causes people

\footnotetext{
${ }^{7}$ Kennedy [147, p. 2] defined it as "non-redundant knowledge that is role-specific and relevant to the task.
} 
to interpret, understand, and evaluate the world differently [136], [151]-[154]. This study follows the latter definition of cognitive distance.

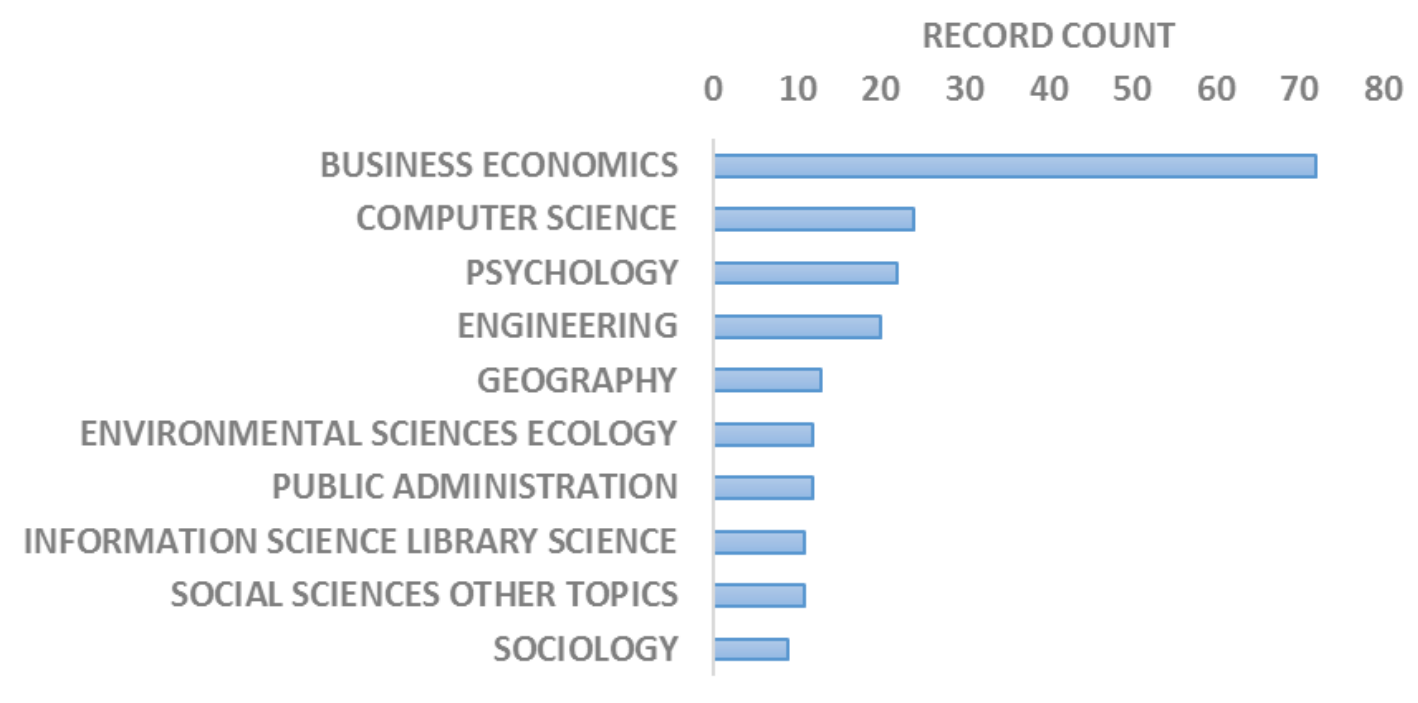

Figure 2.6 The number of papers over top 10 research areas which includes the term "cognitive distance"

People or groups have small/large cognitive distance between them. In the context of group learning or innovation in a group, too small/large of a cognitive distance tend to weaken group learning/innovation performance [136], [151]-[155]. For example, when a cross-functional team is newly organized for a brand new product development (there exists a large cognitive distance between the team members), each member has difficulties in sharing and understanding novel or heterogeneous knowledge. Accordingly, the level of knowledge, novelty or heterogeneity between the members is high. At the same time, the level of mutual understandability (or familiarity) is relatively low. In this case, there exist different understandings and interpretations of given knowledge/information (high equivocality), so that it is hard to share common understandings or to reach consensus in the team. On the other hand, when a team has 
too small of a cognitive distance between the members, the exchange of new knowledge tends to be suppressed because members focus on maintaining relationships and seeking concurrence among them rather than proposing new innovative ideas [136], [156]. In other words, the level of knowledge heterogeneity is low while the level of familiarity is high among the members, enabling the team to make sense of shared knowledge/information or to make agreement rather exactingly (Low equivocality). However, the team tends to have a low interest in achieving or adopting new knowledge/information. In this context, Nooteboom [151], [157] modeled group learning and innovation performance as an inverse U-shaped relation with cognitive distance.

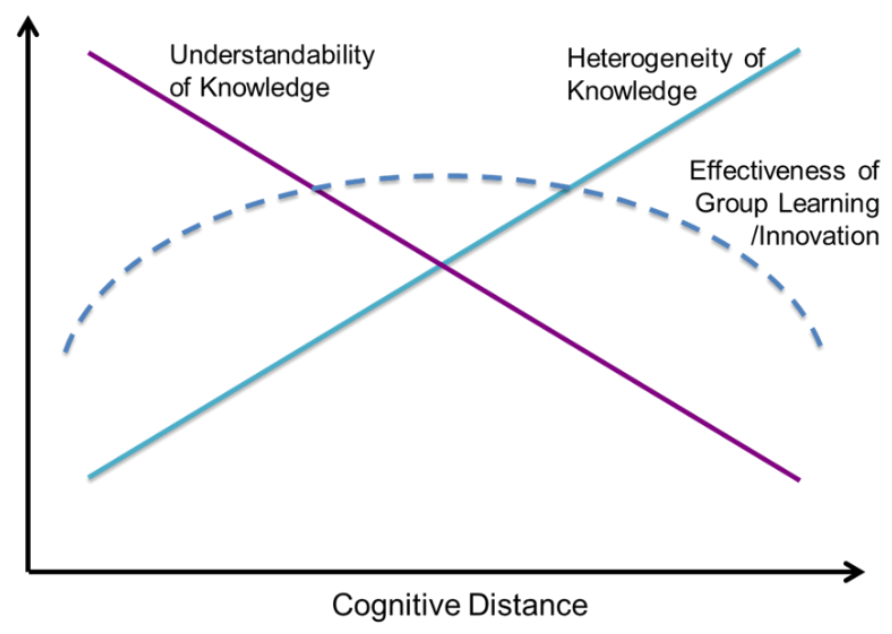

(Source: adapted from [151, p. 279])

\section{Figure 2.7 Model of Cognitive Distance}

Accordingly, the effectiveness of group learning is regarded as the mathematical product of novelty (heterogeneity) and understandability [151]. As depicted in Figure 2.7, the effectiveness of group learning/innovation is low if the cognitive distance is too large or too little. Therefore, to maximize the effectiveness, teams need to manage the cognitive distance between the team members at the appropriate level. 


\subsubsection{Cognitive Distance Reduction}

I propose that large cognitive distance increases the internal distortions in the communication process that are responsible for equivocal message exchanges. The resulting ill-matched understanding of product design factors among participants produces bad concepts, which might lead to disastrous products [28], [158]-[160]. Consequently, it is necessary to reduce the cognitive distance between engineers and market-oriented stakeholders, as depicted in Figure 2.8.

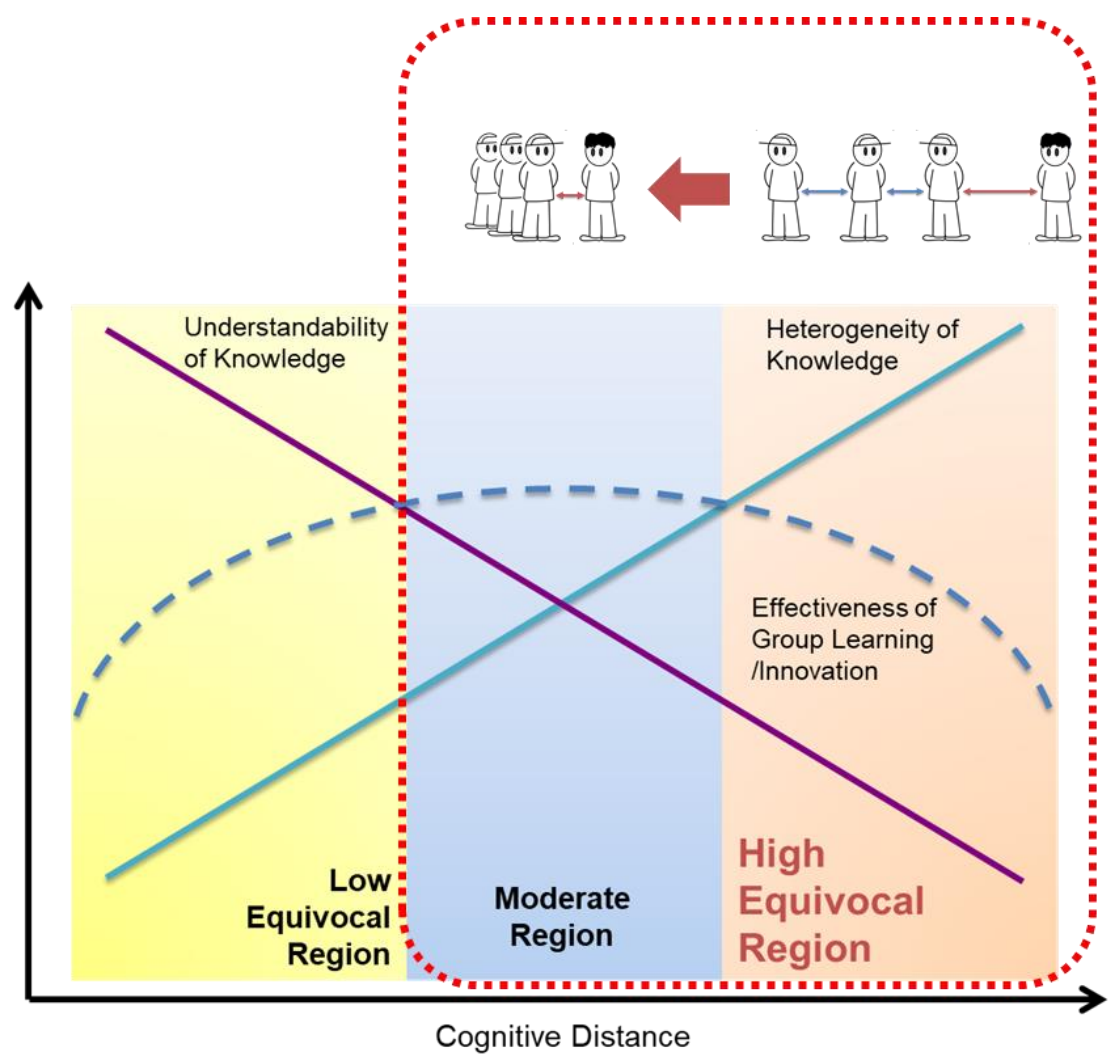

Figure 2.8 Conceptual model of cognitive distance reduction in PCD

This can be achieved by enabling engineers to understand market-oriented information better, for example through better training, through more systematic approaches for getting access to customer knowledge, and through methods that help engineering teams in sharing (or aligning) their mental models with other stakeholders. 
Moreover, product developers may need new ways to engage with customers that lead to more frequent and meaningful interactions, richer descriptions of the customer context, and better transfer of customer insights into NPD projects. In the following sections, I will characterize these needs further and review the existing state of the art.

\subsection{Product Design Factors as an Engineering Challenge}

In this chapter, I will characterize product design factors and investigate the difficulties engineers experience in dealing with them.

\subsubsection{Product Design Factors in Product Concept Development}

Customer preference for a product is affected by various product design factors. These factors, as a rather comprehensive term, encompass all factors that PD engineers can control during the product concept development phase to achieve technical requirements and to enhance customer preference.

In the product development field, the term "attribute" is generally used to describe product design elements related to the characteristics of a product. Definitions of attributes are varied: Krishnan and Ulrich described that attributes, as "an abstraction of a product," are associated with both customer needs and product specifications [161]. Fung, Ren, and Xie differentiate product attributes from customer attributes, defining them as forms of engineering characteristics that generate proper technical actions in response to customer requirements (or Voice of Customer) [20], [162]. Lastly, Vriens and Hofstede defined concrete attributes as "relatively directly observable physical characteristics of a product or service" [163]. Figure 2.9 shows the scope of each of these definitions, as well as the scope of the definition that is used in this research. As depicted 
in Figure 2.9, product design factors in this study encompass all technical but only some non-technical elements of a product because some of the non-technical product design factors are uncontrollable for product design engineers.

\begin{tabular}{|c|c|c|}
\hline \multicolumn{2}{|l|}{ Non-technical } & \multirow[b]{2}{*}{$\begin{array}{l}\text { (Krishnan \& } \\
\text { Ulrich [161]) }\end{array}$} \\
\hline $\begin{array}{l}\text { Customer Needs } \\
\text { (customer attributes, } \\
\text { customer requirements) }\end{array}$ & $\begin{array}{l}\text { Product Specifications } \\
\text { (engineering characteristics, } \\
\text { technical performance) }\end{array}$ & \\
\hline $\begin{array}{l}\text { Customer Attributes } \\
\text { (voice of customer, customer } \\
\text { requirements) }\end{array}$ & $\begin{array}{l}\text { Product Attributes } \\
\text { (the form of engineering } \\
\text { characteristics or feasible } \\
\text { solutions) }\end{array}$ & $\begin{array}{l}\text { (Fung, Ren \& } \\
\text { Xie [162]) }\end{array}$ \\
\hline \multicolumn{2}{|c|}{$\begin{array}{l}\text { Concrete Attributes } \\
\text { (mostly directly observable physical } \\
\text { characteristics of a product or service) }\end{array}$} & $\begin{array}{l}\text { (Vriens \& } \\
\text { Hofstede [163]) }\end{array}$ \\
\hline
\end{tabular}

\section{Figure 2.9 The scope of the concept of "product design factors" corresponding to typical definitions of attributes}

Technical and non-technical product attributes are frequently organized in typologies: According to Crawford and Benedetto, product design factors are comprised of three different elements: benefits, functions, and features [164, p. 151]. Benefits refer to definable advantages, improvements, or satisfaction for the customer achieved by one or more features of the product [164], [165]. Functions are associated with what the product does or how it works. Lastly, features are related to what the product consists of. These three-type attributes are usually linked organically and sequentially with each other [164], [166]. In other words, one or more features allow a function, and the function provides one or more benefits in combination with other functions or features. Similarly, 
Crawford and Benedetto described, "Technology permits us to develop a form that provides the benefit" [164, p. 103].

Srinivasan et al. divide product design factors into three design elements: functionality, aesthetics, and meaning. Functionality, as mainly the engineering function's domain, results from the combination of technical features that satisfy customers' functional needs for a product. Aesthetics, as mainly the industrial design's domain mainly, comes from sensorial characteristics (e.g., appearance, sound, touch, and feel) that enhance the appeal of a product by evoking customers' feelings. Lastly, meaning refers to representative images (e.g., brand, packaging, and distribution) of a product, which are formed by the interaction between firms and customers [71]. Similarly, Homburg, Schwemmle, and Kuehnl categorize product design factors, which they call product design dimensions, into three design dimensions: functionality, aesthetics, and symbolism. They investigate which dimensions affect customer purchase intention, word of mouth, and brand attitude [167]. They conclude that all three dimensions influence customer behavior directly or indirectly.

Additionally, Luo, Kannan, and Ratchford differentiate subjective characteristics of a product from objective ones to develop a formal model that enables product designers to incorporate subjective characteristics in product design and evaluations [14]. Zeithaml divides product design factors into intrinsic and extrinsic factors to conceptualize customer perceptions of price, quality, and value [166]. Moreover, focusing on the psychological aspects of customers' buying decision in examining complex, expensive high-tech products, Shaw, Giglierano and Kallis organize product design factors into 
tangible and intangible ones [168], [169]. Wang, Wang, and Wang classify product design factors according to the length it takes to develop them [170]. Core attributes, referring to the long cycle in product development, would promote absolute competitiveness in the market. On the other hand, extension attributes, referring to the short cycle, would promote relative competitive.

Lastly, Kano et al. suggested a method to determine types of functional or emotional customer needs by classifying product design factors into five categories: Must-be, One-dimensional, Delighting, Indifferent, and Reverse [165], [171]-[173]. Table 2.5 summarizes the description of the Kano categories, and Figure 2.10 shows a graphic representation of the Kano method.

Table 2.5 Description of the Kano categories

Category Description

Must-be
(Basic, Expected)

One-dimensional

(Performance, proportional)

\section{Delighting}

(Attractive, Exciting, Value-Added)

Indifferent

Reverse
These product features cannot increase satisfaction. And, if performing less than average, dissatisfaction will be increased.

The better the product performs, the more satisfied customers and vice versa.

The feature provides extra product satisfaction for customers.

The feature does not provide either satisfaction or dissatisfaction.

The attribute causes customers annoyance/dissatisfaction. 


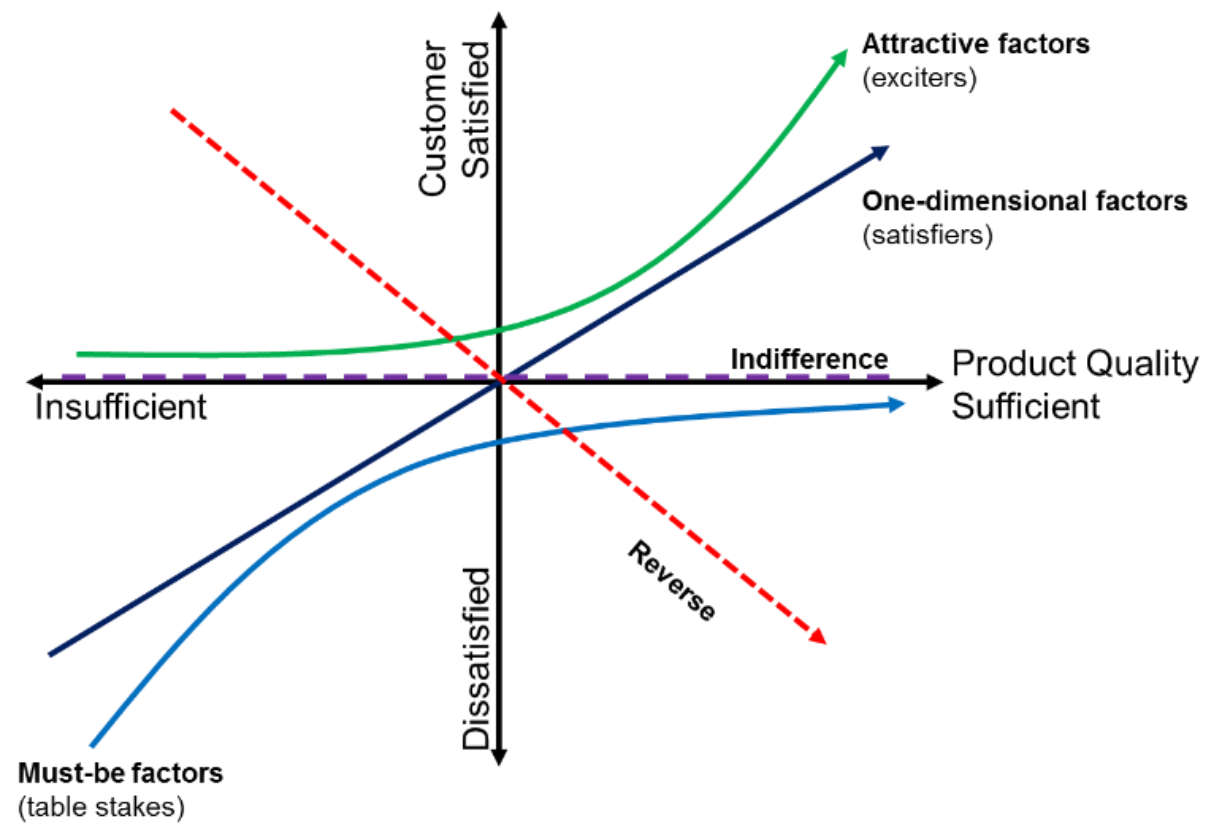

(Source: adapted from [165], [172])

Figure 2.10 Graphical representation of the Kano method

Figure 2.11 summarizes the above discussion of the typologies of product design factors. As the overview shows, there are factors that are intangible, subjective, and very much part of the customer's perception. For example, nontechnical-aspects, such as customers' loyalty, perceived image of a product give a product "meaning" that may exist largely independent of the specific technical factors of the product. On the other hand, technical factors can convey non-technical, subjective factors such as product image or brand identify. It is, therefore, important to consider the relationships between product design factors from a holistic and systemic point of view. 


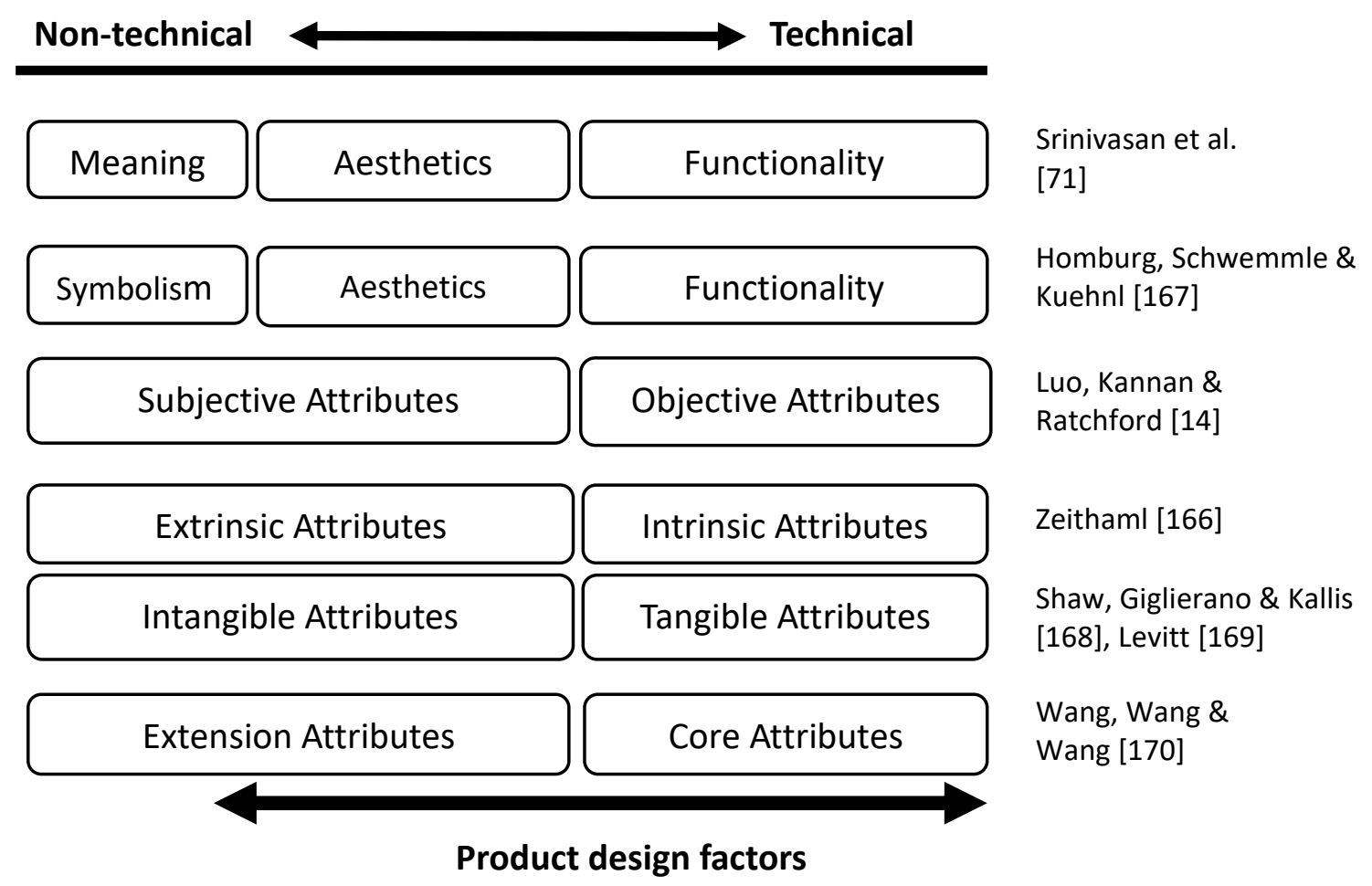

Figure 2.11 Typologies of product design factors in the literature

\subsubsection{Subjective Design Factors}

Considerable research has tried to understand the impact of non-technical product design factors on customer preference and points out that actors might interact [14], [71], [166]-[170], [174], [175]. For instance, Luo et al. show that subjective attributes are affected by objective attributes, and both attributes influence customers' purchase intention directly or indirectly [14].

Additionally, as user experience is emphasized to strengthen the product competitiveness and the differentiation from others [176]-[179], researchers have tried to convert customers' feeling into engineering design factors [180]-[184]. For example, Kansei Engineering ${ }^{8}$ allows PD engineers to translate the consumer's feeling into the

8 "Kansei" means psychological feeling or image of customers regarding a product [180], [185], [292]. 
product design elements [185]. In practice, sensory factors often represent a distinctive characteristic that differs from the characteristic of other products and increase customer preference. Examples are the closing-door sound of a Rolls Royce [183], or the exhaust sound of a car [185]. As such, engineers in consumer-product industries are already trying to translate sensory experiences into design factors and to treat them as engineering problems.

In spite of that, there is only limited research that aims to explicitly model the impact of subjective attributes on customer preference because of measurement challenges. The challenge is caused by heterogeneity across individuals, who may perceive different sensory inputs differently. Accordingly, there are not generalized measures for evaluating subjective characteristics of a product [14], [174]. As a result, while engineers typically understand clearly how the objective characteristics of their technology alternatives fulfill technical requirements, they might be uncertain about the impact of subjective characteristics on customer preference.

\subsubsection{Structural Complexity}

Along with modeling the impact of subjective design factors with objective ones, it is necessary to capture complex causal relationships among product design factors. Complexity refers to a quality of an artifact with many interdependent components and attributes which impede product developers' and customers' understanding of the whole artifact [186], [187]. Similarly, Stevens explained, "Complexity occurs when too many parts of a system interact in a non-simple way. The link between decision and effect is difficult to forecast because of the unpredictable course of the interactions between subparts" [94, p. 433]. Additionally, he included complexity as the main cause of 
fuzziness during FEE. In designing a product, structural complexity leads to difficulty in describing and analyze the relationships between product design factors [188].

Product design factors are interconnected with each other [69], [96], [97]. Typically, the interconnection, or coupling, among physical features and functions of a product or a system is well-known [64], [98]. Moreover, some aesthetic factors often influence the characteristics of other functional factors. As Luo et al. mentioned, the objective and the subjective design factors jointly influence the customer preference of a product [14]. In some cases, the appearances of products determine the sizes of sub-modules or parts as well as aesthetic and experiential results. For instance, in designing a smartphone, the thickness of a phone is regarded as a determinant design factor for users' feelings. To reduce the thickness, the thickness of the battery should also be thin, which leads to a reconsideration of the capacity of the battery because the capacity is interrelated with the volumes of a battery. For another example, the stiffness of the case should be strengthened by reconsidering materials or the structures because the change in thickness physically affects the stiffness. This structural complexity of products impedes product development teams from assessing the impact of a concept change on all other design factors or the customer value of a target product, causing teams to miss or ignore invisible effects and unintended consequences of product concept decisions.

\subsubsection{Summary: Challenges of Dealing with Product Design Factors}

The sections above outline several challenges PD engineers during product concept development. Their task is to determine technical design factors, yet there are many nontechnical factors that also determine product attractiveness. Many of these non-technical factors are perceived differently by different people, and difficult to measure. Subjective 
design factors can also determine how a technical design factor is received and contributes to customer preference.

On the other hand, technical design factors can evoke subjective design factors and, for example, cause delight or frustration, provide meaning, or convey brand image. The interdependencies between design factors lead to complexity, which can cause product development teams to overlook or overestimate the impact of single factors. In order to prevent or minimize such errors, it is important to analyze the impact of each product design factors on customer preference, given its total direct and indirect effects. PD teams consequently need systematic methods for analyzing the interconnected causal relationships between design factors and customer preference and for sharing a holistic, system-oriented view of the development project that identifies the causal relationships among product design factors [73], [189]. By doing so, they can develop successful product concepts by identifying technology alternatives that maximize customer preference before freezing product concepts or starting the full-fledged design phase [11]. The following section will explore to what extent such methods exist.

\subsection{The State-of-the-Art of Product Concept Development Methods}

Various NPD tools and marketing frameworks emphasize the need to incorporate customers' contributions and insights in the so-called the FFE of NPD [87], [92], [96], [97], [100], [102], [109], [190], [191]. Current methods for NPD recognize that engineers typically work in cross-functional development teams, including marketing. In these teams, marketing is responsible for absorbing and interpreting customer knowledge. Accordingly, many studies in the last decade have suggested methodologies and frameworks which enhance cooperation between marketing and engineering divisions 
[15], [25], [26], [53], [55]-[57], [60]-[62]. Interestingly, the majority of this research deal with the conflict between marketing and engineering that arise from communication problems and lack of mutual understanding [26], [53], [54], [56], [58], [62]. These problems are caused by equivocality, which is defined as difficulty in exchanging different subjective views (or reducing the cognitive gap) between individuals [108], [120]. This problem not only exists between marketing and engineering but also between customers and NPD teams [23], [121].

To resolve the communication challenges between PD engineers and marketing/customers, research suggests a variety of managerial and organizational approaches [8], [9], [26], [53]-[58], [60]-[62], [192]-[195]. These approaches focus on reducing communication problems and improving mutual understanding in interdisciplinary teams through, among others, seniority and skillfulness of leadership, leadership commitment, a culture of mutual respect, frequent and high levels of communication, and co-location.

Process-oriented approaches, on the other hand, emphasize the use of concept development process tools to systematically explore the relationships between customer requirements and design characteristics. The dominant method in this context is quality function deployment (QFD). QFD has been widely adapted by a variety of industries since its invention and introduction in the 1990's. According to Bouchereau and Rowland [13], this method enables multifunctional teams to bring large amounts of verbal data and to construct relations between customer needs and design characteristics in a logical way. This occurs in a matrix format, commonly characterized as "House of Quality." QFD 
helps to reduce development time and costs and to increase quality [13], [77]. Furthermore, QFD is applicable not only for product development but also for service and process development [196].

Meanwhile, to solve the issues caused by the structural complexity of product design factors, research suggests to decompose and simplify the intricately interwoven product design factors into system chunks, functions, or sub-systems [197]-[199]. One such approach is the axiomatic design approach (ADA), which helps product development teams to formalize design problems, conceptualize technological alternatives, remove bad design ideas and select the best concepts in the concept development phase by providing a systematic and scientific basis for design decisionmaking [200]-[202]. The fundamental principles of this approach consist of two axioms: the independent and the information axioms. The first axiom refers to maintaining the independence of the functional requirements, and the second axiom is to minimize the information content of the design while satisfying the first axiom. Based on these two axioms, the method leads development teams to decompose a high-level abstraction (i.e., customer needs and functional requirements) into detailed design factors in low levels (i.e., features and parameters). Consequently, the design decisions made in the higher levels affect design factors in the lower level (Please see Appendix A for further details).

Lastly, some methods provide visual representations of relationships between product design factors graphically by utilizing a network of empirical models with historical market data [12] and a neural network with engineering models [11], [203]. Interestingly, the visualized representations of these methods support development teams 
in intuitively and effectively understanding complicated systems in which product design factors are interwoven with each other. Additionally, it is possible for development teams to obtain quantitative results because the relationships between design factors are defined with engineering mathematical functions, or because historical market data is utilized with engineering design constraints and mapping functions between specifications and attributes of a target product.

Table 2.6 summaries tools and methods for product concept development. They all decompose the complexity of the product concept and aim to model the relationships between the elements. 
Table 2.6 Decomposition and Modeling in the product concept development phase

Name of

Tool/Method

Quality Functional

Deployment (QFD)

\section{Description}

- A development process to convert qualitative customer

knowledge to quantitative design parameters to achieve customer satisfaction on a target product
Key

Reference

[3], [63]-[65],

[77]

[5], [11], [203]

which includes engineering models designers are familiar with

to set realistic target specs and to consider coupling among design characteristics

Fuzzy QFD

- To deal with ambiguous customer requirement information in

conventional QFDs, subjective linguistic variables are

represented as fuzzy numbers.

Analytic Target
Cascading (ATC)

- Based on conjoint choice data, A system optimization tool connecting consumer preference with engineering capabilities

[15], [68]

to maximize the profit from a product

- This tool assumes consumer preferences to be homogeneous.

Analytical
Hierarchy Process
(AHP)+TOPSIS

Fuzzy Analytic

Network Process

(Fuzzy ANP)
- A framework that integrates the analytical hierarchy process (AHP) and the technique for order preference by similarity to ideal solution (TOPSIS) to assist designers in identifying customer requirements and design characteristics for the customer-driven design process design alternatives developed in an NPD environment to reach

[70], [204] to the best one satisfying both the needs and expectations of customers and the engineering specifications of the company

[69]

\begin{tabular}{|c|c|c|}
\hline $\begin{array}{l}\text { Data-Driven } \\
\text { Optimized } \\
\text { Engineering } \\
\text { Specification }\end{array}$ & $\begin{array}{l}\text { - A methodology that determines engineering specifications by } \\
\text { balancing market environments and engineering feasibility in } \\
\text { the early stages of the development processes using statistical } \\
\text { analysis of historical data }\end{array}$ & [12] \\
\hline $\begin{array}{l}\text { Total Product } \\
\text { Design Concept } \\
\text { (TPDC) }\end{array}$ & $\begin{array}{l}\text { - An integrated, customer-based framework for product design } \\
\text { consisting of three elements - functionality, aesthetics and } \\
\text { meaning - and the links between these elements and } \\
\text { customers' experience with a product }\end{array}$ & [71] \\
\hline $\begin{array}{l}\text { Bayesian Causal } \\
\text { Map }\end{array}$ & $\begin{array}{l}\text { - A causal map, i.e., a network-based representation of an } \\
\text { expert's cognition with the characteristics of a Bayesian } \\
\text { network, a graphical representation of an expert's knowledge } \\
\text { based on probability theory }\end{array}$ & $\begin{array}{l}{[72],[73],} \\
{[205]}\end{array}$ \\
\hline $\begin{array}{l}\text { Product Concept } \\
\text { Generation and } \\
\text { Selection (PCGS) }\end{array}$ & $\begin{array}{l}\text { - An analytical approach to determine design specification, in } \\
\text { particular determining targets of design attributes or } \\
\text { engineering characteristics using fuzzy c-means algorithm } \\
\text { - The overall customer satisfaction degree (OCSD) indicates } \\
\text { the maximum customer satisfaction }\end{array}$ & [74] \\
\hline $\begin{array}{l}\text { Axiomatic } \\
\text { Approach }\end{array}$ & $\begin{array}{l}\text { - A design framework to generate design parameters by } \\
\text { analyzing customer attributes and functional requirements } \\
\text { systematically based on simplifying complex problems }\end{array}$ & [200], [201] \\
\hline
\end{tabular}




\subsection{Effectiveness of Existing Decomposition and Modeling Methods}

Rather than providing a comprehensive review of each approach in Table 2.6, I will highlight some of the most pertinent problems:

QFD and its newer modifications have critical issues in knowledge processing because the complexity of QFD increases when customer needs and design characteristics increase [13], [64]. As a result, constructing QFD may turn into overly time-consuming work. Any modification and update (e.g., the addition of customer needs that were initially not included) force teams to re-construct large parts of "Houses of Quality." Moreover, QFD provides a static snapshot of customer needs and hardly ever reflects customer needs over a wider time frame [13], [15], [64].

Similar problems exist for ADA: It is a useful method for identifying inherent causes of a design problem and for evaluating the quality of product concepts (i.e. if they follow the axioms or not). However, the approach quickly becomes cumbersome when the target product is expected to have many functional requirements and design parameters. In these cases, the second axiom (Information Axiom) requires information about the probability of achieving functional requirements with design parameters, which is impractical for real problems [206]. Also, ADA is inherently a top-down approach: while the impacts of higher-level design changes on customer value are predictable, it is difficult to assess the impacts of lower levels of design factors. Also, when new customer requirements are added during the product concept development phase (e.g. because market research leads to new insights), development teams need to check changes between functional requirements and design parameters throughout the entire system and ensure that no violations of the two axioms occur. 
QFD and ADA (and many of the methods identified in Table 2.6) thus share that they require considerable effort (and data) and are difficult to quickly update when new information becomes available. The data-intense, heavy-weight process makes them illsuited for very early stage product concept development for new products, when multiple concepts may be explored in parallel, concepts are still ill-defined, and little or no historic data exists. It also makes them relatively inaccessible for engineering teams, who do not easily see how a particular product design factor impacts customer preference.

In sum, based on the aforementioned advantages and disadvantages of typical methods, it is possible to summarize the requirements of methods supporting product development teams in assessing alternative product concepts and their respective impact on customer value in the concept development phase. Above all, methods for concept development and evaluation need to allow teams to process information from several disciplines and from customers by mitigating the negative effects of cognitive distance within the teams. Methods should function as group learning tools that guide team members towards developing a shared understanding of product design factors that does not differentiate between technical and non-technical factors and helps the evaluate product cocnepts by facilitating interactive communication and exchange of subjective interpretation within the teams [1], [58], [73], [120], [126], [189], [207], [208]. Secondly, development teams should understand the design factors in the context of other factors. Therefore, the methods, as modeling tools, need to model all causal relationships between design factors, enabling teams to capture associative interrelation between design factors [5], [11], [203]. Additionally, the models underlying the method need to be easy to updated by excluding or adding design factors from the model during the concept 
development phase [62], [122]. Lastly, the methods should allow teams to assess how a change impacts all other design factors and future customer value by testing various concepts, as scenarios, combined with different design factors. Moreover, teams can simulate potential customer responses to additional functions, which enables firms to provide rapid updates to customers [209], [210].

Based on the requirements summarized above, typical product concept development methods are evaluated in Table 2.7. Most methods help teams facilitate group learning to some extent and support teams in bridging functional design factors and customer needs. However, some methods have limitations in enabling teams to have interactive communication and exchange of subjective interpretations concerning product design factors and their relationships. Furthermore, using typical methods, it is difficult or impossible to model the entire relationships between all product design factors: Some methods can model a limited number of relationships but frequently have difficulty representing engineering perspectives, while others do not support analyzing causal relationships. Lastly, the majority of methods rank pre-determined concepts rather than providing ways to test concepts as various combinations of design factors through simulation. The lack of simulation capapbilites furthermore make it difficult to analyze how sensitive customer value responds to changes of each design factor. This makes it difficult to asses decisions on engineering trade-offs. 
Table 2.7 Evaluation of typical methods supporting product concept development

\begin{tabular}{|c|c|c|c|}
\hline \multirow[b]{2}{*}{ Name of Tool/Method } & \multicolumn{3}{|c|}{ Methods for the concept development phase need to support ... } \\
\hline & group learning & $\begin{array}{c}\text { modeling causal } \\
\text { relationships } \\
\text { between product } \\
\text { design factors }\end{array}$ & $\begin{array}{c}\text { scenario testing of } \\
\text { concepts }\end{array}$ \\
\hline $\begin{array}{l}\text { Quality Functional } \\
\text { Deployment (QFD) }\end{array}$ & $\Delta$ & $\Delta$ & - \\
\hline $\begin{array}{l}\text { HOQ + Engineering } \\
\text { Models }\end{array}$ & $\sqrt{ }$ & $\Delta$ & $\sqrt{ }$ \\
\hline Fuzzy QFD & $\Delta$ & $\Delta$ & - \\
\hline $\begin{array}{l}\text { Analytic Target } \\
\text { Cascading (ATC) }\end{array}$ & $\Delta$ & - & - \\
\hline $\begin{array}{l}\text { Analytical Hierarchy } \\
\text { Process (AHP)+TOPSIS }\end{array}$ & $\Delta$ & - & - \\
\hline $\begin{array}{l}\text { Fuzzy Analytic Network } \\
\text { Process (Fuzzy ANP) }\end{array}$ & $\Delta$ & $\Delta$ & - \\
\hline $\begin{array}{l}\text { Data-Driven Optimized } \\
\text { Engineering } \\
\text { Specification }\end{array}$ & $\Delta$ & $\Delta$ & $\sqrt{ }$ \\
\hline $\begin{array}{l}\text { Total Product Design } \\
\text { Concept (TPDC) }\end{array}$ & $\Delta$ & $\Delta$ & - \\
\hline Bayesian Causal Map & $\Delta$ & $\Delta$ & $\Delta$ \\
\hline $\begin{array}{l}\text { Product Concept } \\
\text { Generation and } \\
\text { Selection (PCGS) } \\
\end{array}$ & $\Delta$ & - & - \\
\hline Axiomatic Approach & $\Delta$ & $\Delta$ & - \\
\hline
\end{tabular}

\subsection{Requirements of a New Method}

In order to overcome the limitations of the present methods above, this work aims to develop a new approach for supporting product development teams in assessing alternative product concepts and their respective impact on customer value. The new method should have the following characteristics: 
- Facilitating group learning: Guide the PD team to develop a shared understanding of product design factors and their impact on customer preference by facilitating communication and exchange of subjective interpretation within the team

- Modeling causal relationships between product design factors: Help PD teams understand design factors in the context of other factors by explicitly modeling interdependencies.

- Scenario testing of various concepts: Enable teams to simulate product concepts to assess their impact on customer value. Allow for easy updates of these simulations when product design factors change. 


\section{RESEARCH APPROACH}

\subsection{Research Gaps}

From the previous comprehensive literature review, research gaps are identified below;

\section{Current Methods...}

RG1 ....are limited in guiding each member of teams in sharing a common understanding of product design factors.

RG2 ...have no or limited ability to model causal relationships between product design factors systematically and holistically.

RG3 ...mostly lack the ability to simulate scenarios as product concepts.

\subsection{Research Objective and Questions}

Based on the gaps identified above, this research concentrates on developing a fuzzy cognitive map (FCM)-based method (called Cognitive Distance Reduction Method, CDRM) that allows product development engineers to assess systematically, holistically, and iteratively alternative product concepts and their respective impact on customer value by modeling them as combinations of product design factors. Consequently, product development teams identify and select product concepts that achieve high customer value, given existing constraints. In this context, the new method needs to be capable of reducing the cognitive distance between market-oriented stakeholders (including customers) and engineers in the product concept development phase, allowing product development engineers to be involved actively in building a model that give them deep insights into customer needs. Furthermore, this method enables engineers to simulate the impact of engineering decisions on the fulfillment of customer needs, which provides a tool to 
create and select product concepts for them. This leads to the five research questions identified below;

RQ 1. How can the cognition of market-oriented stakeholders and product development engineers be modeled?

RQ 2. Is it possible to integrate the separate cognitive models of market-oriented stakeholders and PD engineers?

RQ 3. How can alternative product concepts (i.e., combinations of design characteristics) be represented in the model as alternative input scenarios?

RQ 4. How can the outcomes of alternative input scenarios be used to determine the best product concept alternative?

RQ 5. Does CDRM result in an improved and shared understanding of product design factors among product development team members?

Figure 3.1 summarizes the overview of this dissertation. 
Current methods are limited in guiding PD engineers to sharing common understanding of PDFs with PD teams.
Current methods have no or limited ability to model causal relationships between PDFs systematically and holistically.

Current methods mostly lack the ability to simulate the impact of product concepts on customer preference.

\section{Research Gaps}

To develop a fuzzy cognitive map (FCM)-based method (called Cognitive Distance Reduction Method, CDRM) allowing product development engineers to assess systematically, holistically, and iteratively alternative product concepts and their respective impact on customer value by modelling them as combinations of product design factors. With the method, PD teams identify and select product concepts that achieve high customervalue, given existing constraints.

Research Objective
How can the cognition of market-related stack holders and PD engineers be modelled?

Is it possible to integrate the separate cognitive models of market-related stakeholders and of PD engineers?

How can alternative product concepts be represented in the model as alternative input scenarios?

How can the outcomes of alternative input scenarios be used to determine the best product concept alternative?

Does CDRM result in an improved and shared understanding of product design factors among product development team members?

Research Questions

Figure 3.1 Overview of the dissertation 


\section{RESEARCH METHODOLOGY}

The dissertation research results in a novel method, the cognitive distance reduction method (CDRM), which improves product concept development by helping product development engineers (PD engineers) understand how technical product design factors (PDFs) impact customer value. To develop and evaluate CDRM, the research was undertaken in five stages, as depicted in Figure 4.1.

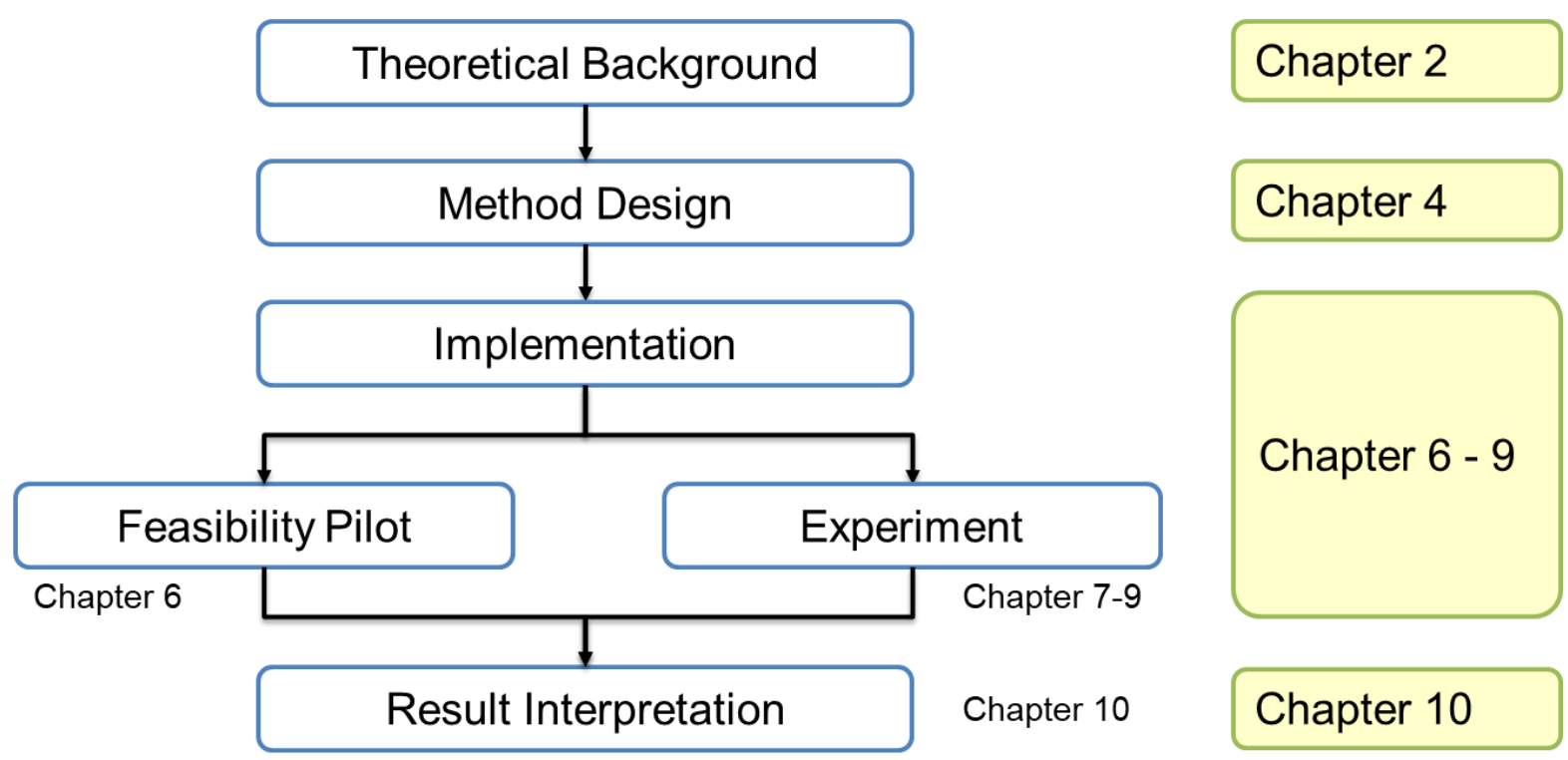

Figure 4.1 Five stages of developing and evaluating CDRM

The first step, literature review, is documented in Chapter 2: it identifies the challenges associated with cognitive distance in product concept development, reviews state-of-the-art methods, and develops the requirements for CDRM. Step 3 is the actual design of the CDRM method: CDRM uses Fuzzy Cognitive Maps for data acquisition, modeling, and simulation. It consists of four major modules, namely: (1) basic concept elicitation, (2) modeling (model-building and validation) (3) simulation, and (4) interpretation of results \& concept selection. As part of step 2 (methodology development, 
I developed each of these modules. In step 3 (implementation), I applied and tested the modules through several small-scale experiments, before the thesis proposal was defended (presented as "preliminary studies") and through a group experiment, after the defense of the thesis proposal (presented as "experiment"). In the experiment, a group of development engineers used CDRM to develop and select concepts for a consumer product. The strategy of implementing CDRM in several smaller-scale experimental approaches, rather than a full implementation in a real-world development team, occurs in response to practical constraints: The concept development phase of a new platform product is highly confidential to protect plans from competitors and time-critical so that R\&D managers are reluctant to test unproven methods. Also, projects can stretch out over months or years and often include multiple global locations. This made a real-world implementation infeasible. However, I used the learning from each partial and experimental implementation and made suggestions for refining CDRM. This analysis occurs in step 4 (Result Interpretation).

The remainder of this chapter is structured as follows: Chapter 4.1 introduces Fuzzy Cognitive Mapping (FCM), which provides the methodological backbone for CDRM. This is followed by a high-level overview of the five CDRM modules in Chapter 4.2. Lastly, the validation strategy underlying this dissertation will be discussed in Chapter

\section{3 .}

\subsection{Background: Fuzzy Cognitive Mapping}

This section describes how FCM technique is utilized in modeling the causal relationships between PDFs in CDRM. 


\subsubsection{General Introduction}

FCM are based on Fuzzy Sets and neural network theory and are used to capture people's knowledge and understanding of uncertain cause and effect relationships [211], [212]. FCM-based methods have gained popularity in a variety of fields such as engineering, business, medical science, environmental science, and social science [213][215], as well as in NPD research [216]-[219]. FCM have several properties that make them particularly attractive as a methodological backbone of CDRM: firstly, FCM are easy to comprehend. They are based on a visualization technique, cognitive mapping, that documents human knowledge as simple visuals and in everyday language. Cognitive maps of people's mental models can be created relatively easily through methods such as interviewing, moderated group workshops, and analyzing texts, such as academic and practitioner literature [220], [221]. The resulting cognitive maps are translated into FCM, which are semi-quantitative networks [221]-[224] that can be used for simulation purposes but are nevertheless easy to understand for laypeople. Secondly, FCM are useful for modeling individual and group knowledge: it is easy to combine FCM that are generated by individual members of the group [220], [225], [226] and synthesize them into a group FCM. Updates to knowledge can be quickly implemented because modifications and additions to the map structure are simple [211], [212], [227], [228]. Lastly, FCM are a useful tool for investigating cognitive differences between individuals and groups based on a comparative analysis of their respective FCMs [226], [229], [230]. Taken together, these properties make FCM suitable for capturing subjective knowledge about product factors and customer value and their complex interdependencies. 
Figure 4.2 shows a network (or directed graph) of causal interconnections between nodes. Such graphs are at the core of FCM techniques: ovals, so-called nodes, can represent any system elements of interest. The interconnections (or edges) between nodes denote causalities. For example, node B increases when node A increases; therefore, the relationship between the two nodes is positive. On the other hand, if node D decreases when node $\mathrm{C}$ increases, the relationship is negative. A relationship can have a weight, which can be directly assigned as a crisp value in the interval (e.g. $[-1,1]$ or $[0,1])$ or elicited based on the linguistic judgment of subjects (e.g. "low," "quite high," or "extremely high"). In the latter case, the linguistic judgements are either translated into crisp values (comparable to the use of Likert scales) or represented using fuzzy logic [189], [231], [232].

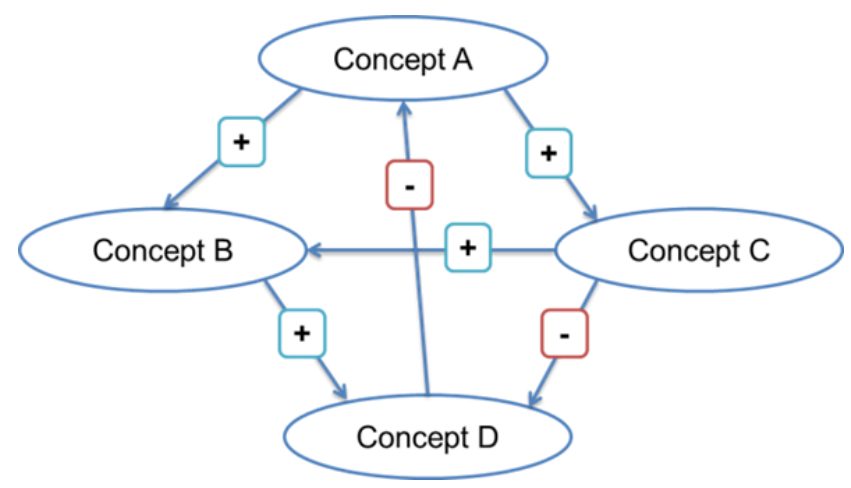

Figure 4.2 Example of an Fuzzy Cognitive Map

FCM graphs can be represented in matrix form. The corresponding adjacency matrix for the above network is represented below:

$$
\mathrm{E}=\left[\begin{array}{rrrr}
0 & +1 & +1 & 0 \\
0 & 0 & 0 & +1 \\
0 & +1 & 0 & -1 \\
-1 & 0 & 0 & 0
\end{array}\right]
$$


To investigate how some nodes in the network change in response to status changes in specific nodes, a state vector that has one row and $\mathrm{n}$ (the number of nodes) columns is multiplied by the adjacency matrix. For instance, if node $\mathrm{A}$ in the above example is activated (i.e., changes its value from 0 to 1 ) while all other nodes are turned off, the matrix is multiplied with the initial state vector below.

$$
S_{0}^{T}=\left[\begin{array}{llll}
1 & 0 & 0 & 0
\end{array}\right]
$$

The initial state vector is chosen to represent a decision ("let's implement A") or a scenario ("factors $\mathrm{X}, \mathrm{Y}, \mathrm{Z}$ are all present").

From the neural network theory inspired by the human nervous system [233]-[235], a stimulus to a node should be strong enough to activate the node, to generate an output signal from the node. Therefore, to transform the level of the output signal into a value within a pre-defined range, a squashing (or threshold) function is. There are several types of squashing functions, such as binary, linear, sigmoid, and hyperbolic tangent functions. These squashing functions are:

- Binary function: $f(x)=\left\{\begin{array}{rr}-1 & \text { for } x<0 \\ 0 & \text { for } x=0 \\ 1 & \text { for } x>0\end{array}\right.$

- Linear function: $f(x)=\left\{\begin{aligned}-1 & \text { for } x \leq-1 \\ x & \text { for }-1<x<1 \\ 1 & \text { for } x \geq 1\end{aligned}\right.$

- Sigmoid function: $\quad f(x)=\frac{1}{1+e^{-\lambda x}}$

- Hyperbolic tangent function: $f(x)=\tanh \lambda x=\frac{e^{\lambda x}-e^{-\lambda x}}{e^{\lambda x}+e^{-\lambda x}}$ 
where $\lambda$ adjusts the saturation level of a node activation. A squashing function converts the multiplied values of the adjacency matrix and a state vector to a new state vector:

$$
S_{t}=f\left(E \cdot S_{t-1}+S_{t-1}\right)
$$

where $t$ denotes an instant. The iteration of this process continues until the state vector reaches stability or a limit cycle: at this point, the multiplication of the state vector (as input) with the matrix results in an output vector that is identical to the input. (Alternatively, the process stops when a stop criterion, typically a very small difference between input and output vectors, is reached). At the final (stable) state, the activation levels of each element in the state vector can be interpreted according to the objective of analysis.

Regardless of the specific modeling project, the general FCM process follows the steps depicted in Figure 4.3 [213]: 


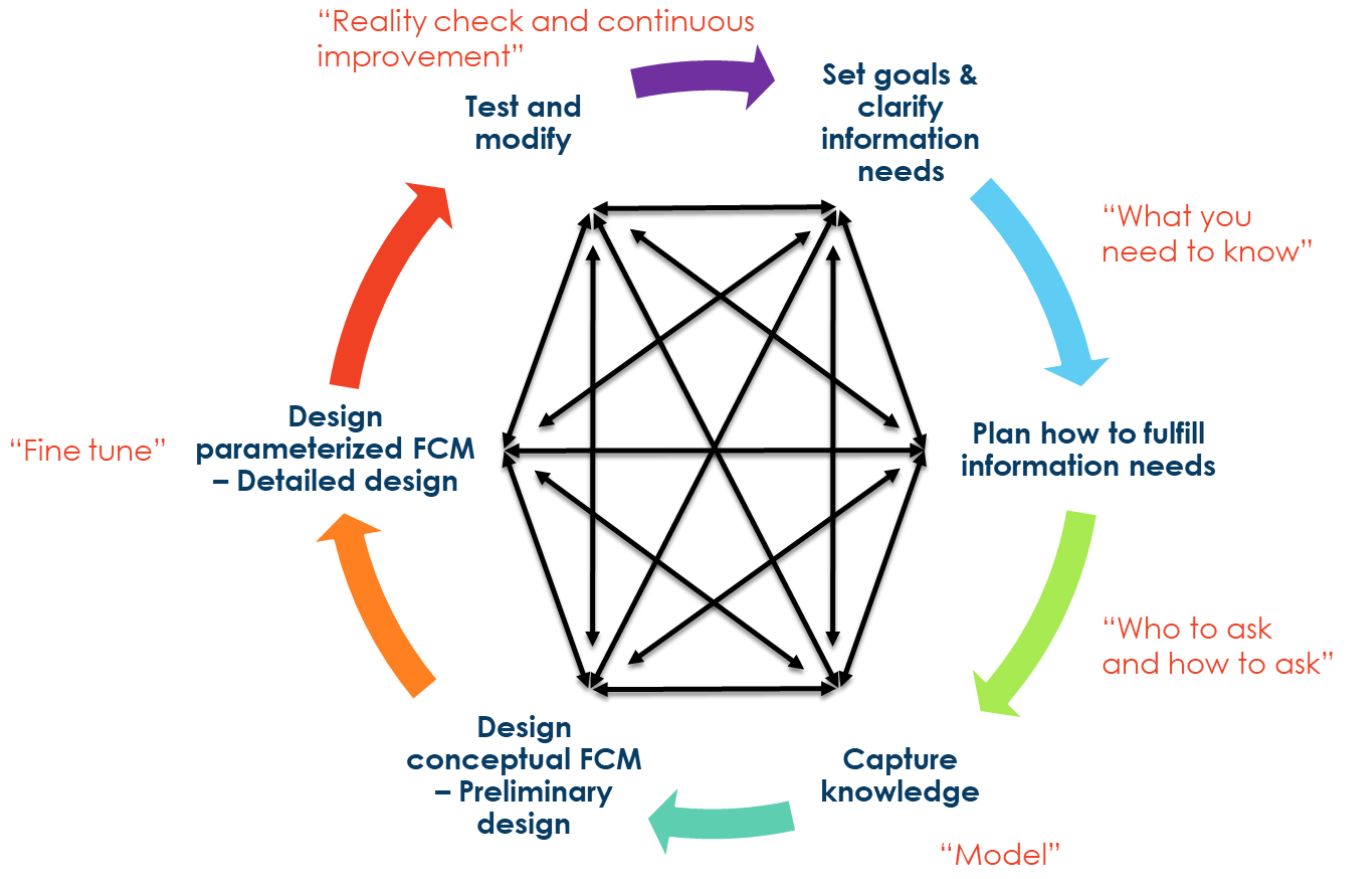

Figure 4.3. Procedure of FCM modeling

The process of fuzzy cognitive mapping is not sequential but iterative until a validated model is obtained. In participatory settings, researchers further strive to reach consensus about the model with all participants in the modeling project [213], [222].

\subsubsection{Aggregated FCM as a group mental model}

An aggregated FCM can be used to represent a group mental model as a representation of how a group understands the world. There are two approaches to develop an aggregated FCM: collecting individual maps and facilitating group modeling [220]. Collecting individual maps enables researchers to characterize collective knowledge more accurately than group modeling because this approach is free from the consideration of group dynamics and provides a more plausible representation of individual mental model while it may be resource-intensive and complicate aggregation [220]. Contrary to collecting individual maps, group modeling allows a community to 
achieve consensus associated with the context of an inquiry or to facilitate group discussion of shared understanding and collective learning, however it has limitations in capturing individual-level understanding of participants [220]. Gray et al. proposed a conceptual model of a spectrum of FCM appropriation in Figure 4.4 [220, p. 42]. Therefore, it is necessary to select a proper approach in developing an aggregated FCM to represent a group mental model considering the advantages/disadvantages of each approach.

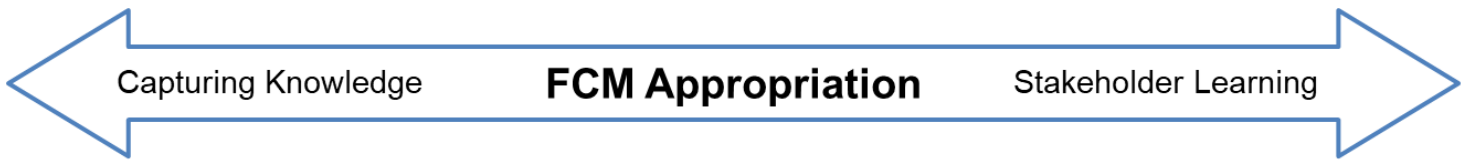

Models are artifacts for decision-making or enhancing system understanding

Combining representations of stakeholders/expert mental models to: (1) reduce uncertainty about a system and (2) compare mental models across groups

\author{
Emphasis Model and modeling process as a tool \\ for social learning \\ Community generated representation of \\ Research knowledge used for planning and \\ Purpose learning, often through participatory \\ scenario development
}

(Source: Adapted from [220, p. 42])

\section{Figure 4.4 Spectrum of FCM appropriation}

According to Amer [236], there are two ways in integrating individual maps: Expert Credibility Weight Method [237] and Averaging Multiple FCMs [238], [239]. Expert credibility weight method utilizes the credibility weights of experts calculated by differences between output vectors of individual maps by arbitrary input vectors based on two assumptions [237, p. 86]:

- The concurrence of an expert with the others implies a high level of expertise 
- The maps contain a sizable measure of expertise

Amer points out that this approach is suitable for a case where achieving consensus is crucial [236] but also criticized that it tends to suppress outlier opinions, which may not always be unsubstantiated but can provide important insights into the systems.

An alternative approach is based on averaging multiple FCMs to create an aggregated map: a union set of nodes (PDFs) in all individual maps is created and the weights of edges is determined through the summation of all edge weights and division by the number of individual maps [212], [213]. This approach is relatively simpler than the expert credibility weight method. However, Kosko [212] warned that number of individual maps (i.e., sample size) affects the reliability of an aggregated map. Therefore, alternatively, borrowing the concept of the credibility weight, each contributor can provide the confidence scale (or credibility weight) of his/her maps in the interval $[0,1]$ [239], [240].

An additional problem arises when all the weights of the edges are averaged without accounting for how often the edges are included in contributing maps: weights for edges that are not included in all individual maps would become overly small. In developing an aggregated FCM, it is, therefore, necessary to consider the existence of common nodes between individual maps [241]. When there are no common nodes among different maps, the adjacency matrix of the aggregated map has the same dimension as the sum of the number of nodes in each individual maps. Also, the weight of each edge between nodes in the contributing maps is maintained in the aggregated FCM. For example, when there are two maps, Map 1 and Map 2, the corresponding adjacency matrices are: 


$$
\boldsymbol{E}_{\text {Map } 1}=\left[\begin{array}{ccc}
0 & 0 & w_{13} \\
w_{21} & 0 & 0 \\
w_{31} & w_{32} & 0
\end{array}\right] \text { and } \boldsymbol{E}_{\text {Map } 2}=\left[\begin{array}{ccc}
0 & w_{45} & w_{46} \\
w_{54} & 0 & 0 \\
0 & w_{65} & 0
\end{array}\right]
$$

The adjacency matrix of the aggregated FCM formed with above two maps is:

$$
E_{\text {Aug }}=\left[\begin{array}{cccccc}
0 & 0 & w_{13} & 0 & 0 & 0 \\
w_{21} & 0 & 0 & 0 & 0 & 0 \\
w_{31} & w_{32} & 0 & 0 & 0 & 0 \\
0 & 0 & 0 & 0 & w_{45} & w_{46} \\
0 & 0 & 0 & w_{54} & 0 & 0 \\
0 & 0 & 0 & 0 & w_{65} & 0
\end{array}\right]
$$

However, in many cases, there are nodes that occur in several of the contributing maps. In the case of such "common nodes," the number of nodes (or the dimension of the adjacency matrix) in the aggregated map is the total number of the elements in the union set of the nodes included in all contributing maps. The weights of the edges between the nodes that exist only in one map are the weights given in the contributing map (see above). The weights of the edges of common nodes are calculated, using the average of all contributing maps that contain the node. For instance, there are two maps which share two nodes, $C_{2}$ and $C_{3}$. Map 1 has three nodes, $C_{1}, C_{2}$ and $C_{3}$, while Map 2 consist of four nodes, $C_{2}, C_{3}, C_{4}$ and $C_{5}$. The corresponding adjacency matrices are:

$$
\boldsymbol{E}_{\text {Map 1 }}=\left[\begin{array}{ccc}
0 & 0 & u_{13} \\
u_{21} & 0 & 0 \\
u_{31} & u_{32} & 0
\end{array}\right] \text { and } \boldsymbol{E}_{\text {Map } 2}=\left[\begin{array}{cccc}
0 & v_{23} & v_{24} & 0 \\
v_{32} & 0 & v_{34} & v_{35} \\
v_{42} & v_{43} & 0 & 0 \\
v_{52} & v_{53} & v_{54} & 0
\end{array}\right]
$$

For example, $u_{21}$ in the adjacency matrix, $\boldsymbol{E}_{M a p 1}$, of the first map represents the weight of the edge from $C_{2}$ to $C_{1}$ while $v_{32}$ in the adjacency matrix, $\boldsymbol{E}_{M a p 2}$, of the second map represents the weight of the edge from $C_{3}$ to $C_{2}$. 
When combined, the weight of the edge between $C_{2}$ and $C_{3}$ is calculated as the average of two weights while the values of other weights are set to the value in the contribution map. Consequently, the corresponding adjacency matrix is:

$$
E_{\text {Aug }}=\left[\begin{array}{ccccc}
0 & 0 & u_{13} & 0 & 0 \\
u_{21} & 0 & v_{23} & v_{24} & 0 \\
u_{31} & w_{32}^{\text {ave }} & 0 & v_{34} & v_{35} \\
0 & v_{42} & v_{43} & 0 & 0 \\
0 & v_{52} & v_{53} & v_{54} & 0
\end{array}\right]
$$

where $w_{32}^{\text {ave }}$, the new weight of the edge is calculated as follows:

$$
w_{32}^{\text {ave }}=\frac{u_{32}+v_{32}}{2}
$$

Khan and Quaddus present a procedure of a group FCM development by collecting individual maps, as depicted in Figure 4.5 [242].

In the procedure, the merged group FCM should be reviewed by the decision group in order to validate it with determining any redundancy of some nodes between individual maps and modifying vagarious or unjustifiable causal links and their weights through interaction between the decision group members.

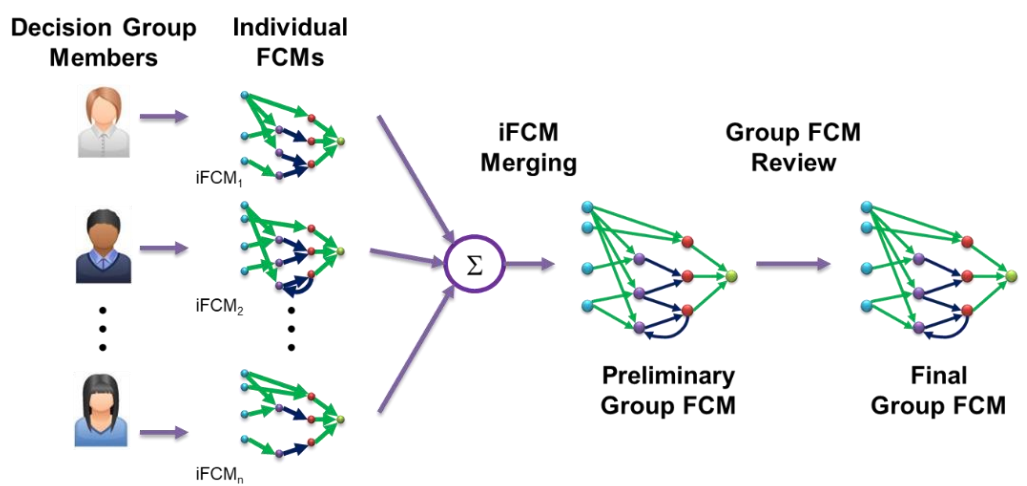

(Source: adapted from [242, p. 474])

Figure 4.5 Development of Group FCM, based on aggregating individual FCMs 
Group modeling (or group model building) is another way to create an aggregated map. As an outcome of group modeling, an aggregated FCM (i.e., an FCM that represents the knowledge of the members of the group) is formulated through social interaction and collective sharing of aspects of participants' individual mental models through multiple meetings, sessions or workshops [220]. To capture a group model and represent it adequately, the process, execution, and role-assignments of group modeling activities are critical[243]-[247]. In particular, Jetter et al. described the preparation and the execution of a modeling workshop for the development of an aggregated FCM [248].

\subsubsection{Fuzzy Cognitive Maps as a Core Part of the CDRM}

Figure 4.6 shows the use of FCM within the CDRM process. The CDRM uses inputs gained from market and technology research to create two types of FCM-based models: Need Map, which is developed by marketing experts to model the relationships between PDFs (features, functions, benefits) and customer preference, and Tech Map, which is developed by PD engineers to reflect how technology alternatives impact the degree to which features and functions are implemented. Figure 4.6 shows the fundamental structure and the overlap between the two types of maps. 


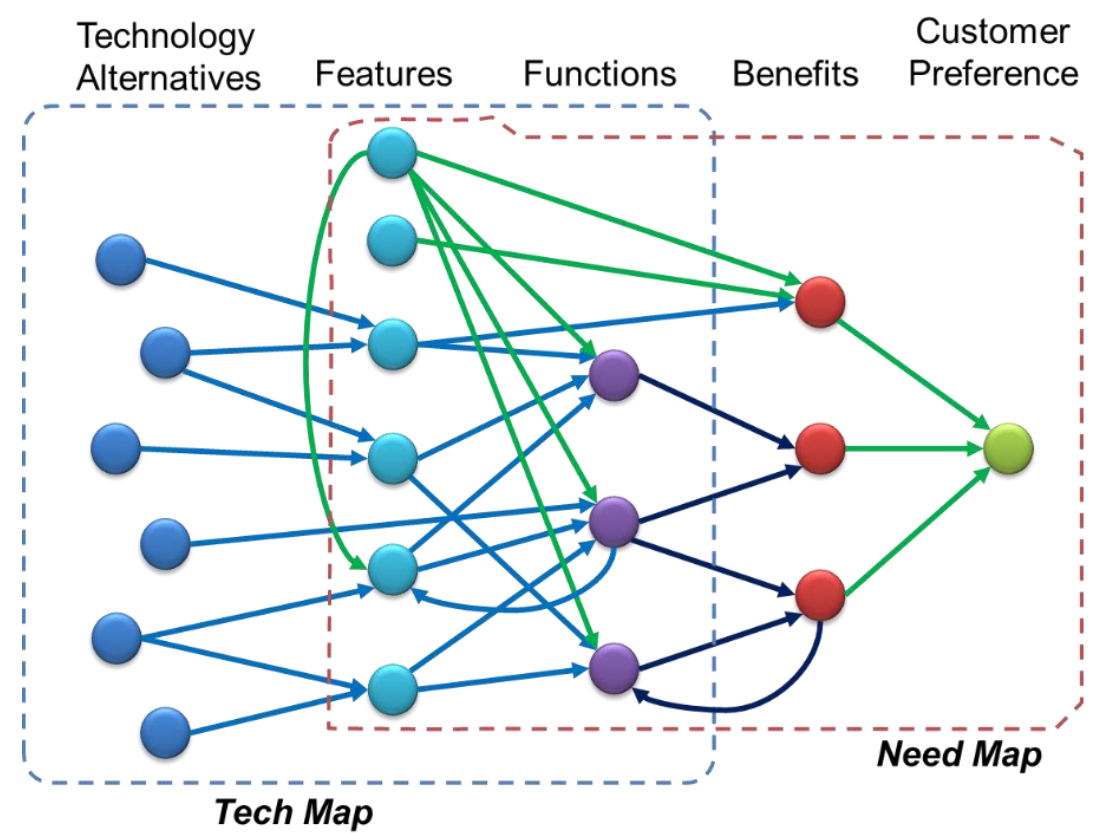

Figure 4.6 Product design factor map as the usage of FCM in CDRM

Both maps utilize data created as part of the regular NPD process, such as technological trend reports, market research data, and requirements analysis. To leverage these data sources for the purpose of creating FCM models, members of the marketing team and PD engineers need to undergo a short training in basic FCM technique so that they can actively contribute and critique the process. The actual CDRM process, however, should be facilitated by a method expert.

The expert should further facilitate the subsequent modeling steps, namely the validation of the Tech and Need Map, their synthesis into an aggregated FCM model, and the model use through simulation. Simulation results will be used by marketing and engineering to develop product concepts. 


\subsection{Process Steps of CDRM}

The generic process of the CDRM is depicted in Figure 4.7. A more detailed description of each step is provided in the following sub-chapters.

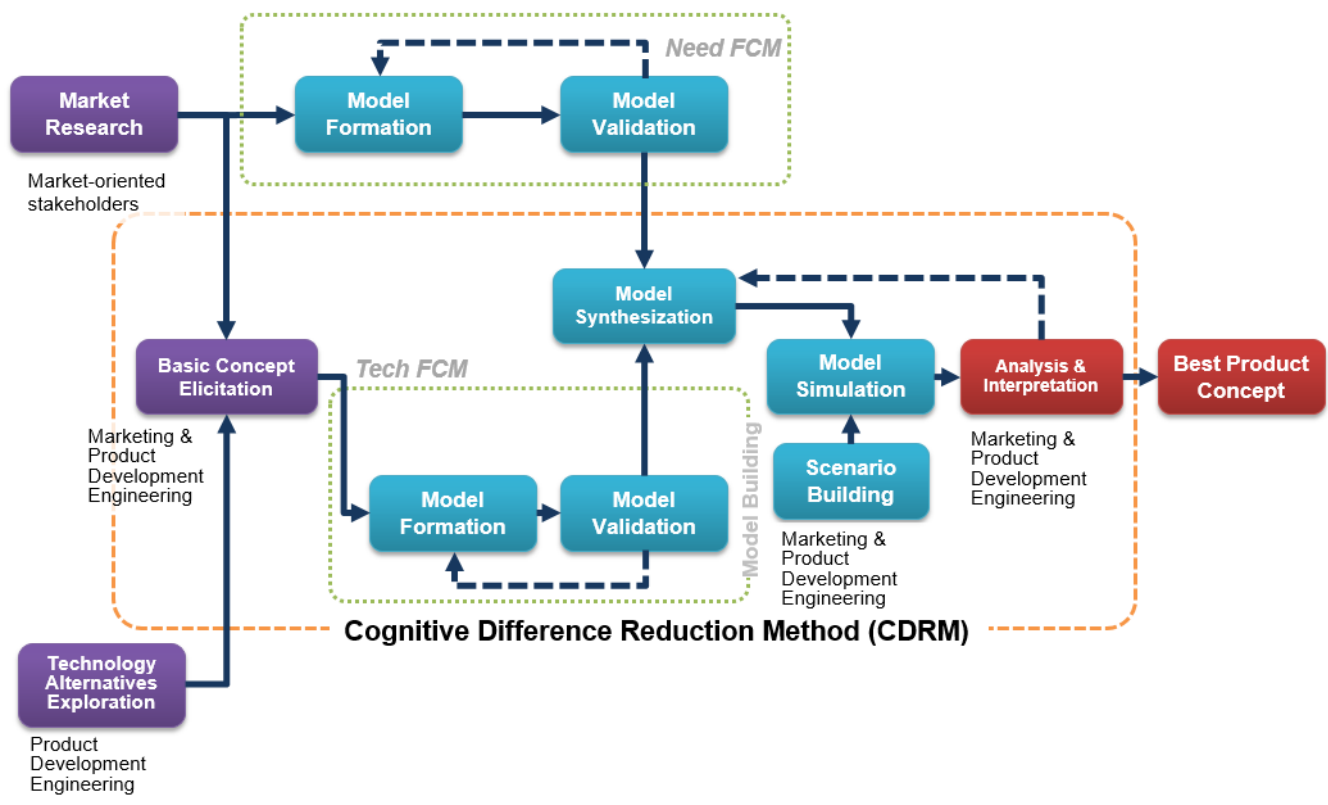

Figure 4.7 Generic process of CDRM

\subsubsection{Basic PDF Elicitation (Step 1)}

The full-fledged process of CDRM starts from the basic PDF elicitation phase as a preparation activity of the modeling workshop. The project team needs to elicit the "start PDF," which is used for modeling Tech Map and Need Map. This step leverages research and documents that are commonly produced in the early planning stages of a new product, namely mission statements [3], user requirements document, market requirements document [249], technical requirements document [250], customer journey [251] and personas[176]. In addition, experts who were involved in the earlier research stages can share their knowledge of PDFs. In eliciting fundamental PDFs, it is advisable that engineers focus their attention on realizable features, functions, and technology 
alternatives for the target product while marketing experts extract information about benefits, desired functions, and features from the documents.

Based on this elicitation step, basic PDF index cards will be developed, as shown in Figure 4.8. Each index card includes the title of a PDF and its description. Titles and descriptions are chosen for maximum clarity to prevent any confusion or difficulties when the contributors use the index cards in the next modeling step. The index cards serve as the basis of the so-called knowledge activation step in mental model elicitation: rather than asking participants to model a complex system right away, which is cognitively demanding, modeling is broken up into two steps: In the first step, participants are supported in identifying elements of the system by giving them index cards with system elements as stimuli. In a subsequent step, they focus on the structure (i.e. the connections between the system elements) [220], [248], [252]. The index cards of PDFs thus serve as the backbone of Tech or Need Maps, and support participants in subsequent steps in identifying the causal relationships between PDFs.

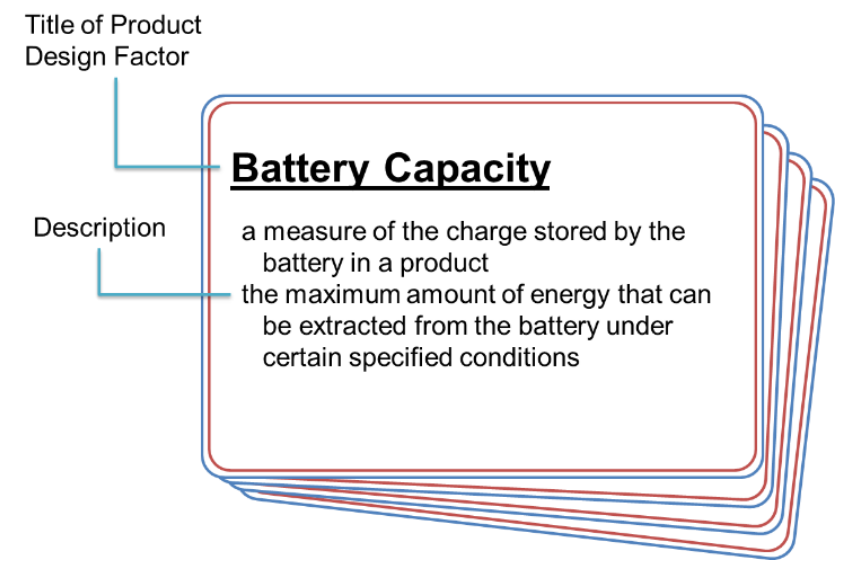

Figure 4.8 A sample image of a PDF index card 


\subsubsection{Model Formation (step 2)}

Modeling occurs in several steps: first, the participants (members of the PD team and, where appropriate, additional technology and market experts) are briefed about the project at hand, including its mission statement, targeted market, and constraints. They subsequently create representations of their individual mental models of the project in the form of causal cognitive maps. A modeler aggregates these individual maps and translates them into an FCM model: one FCM model, generated with inputs from marketing, is Need Map, and a second model, generated with inputs from engineering, is Tech Map. The two FCM models are reviewed and iteratively improved in two separate workshops (one for each model) under the leadership of a facilitator and with members of the PD teams who are knowledgeable on the respective subject matters. The review process serves to function: on the one hand, it ensures that the models are adequate representations of the problem at hand. On the other hand, it gives the PD team members to reflect on their individual mental models and to become aware of the "bigger picture." Each of the main steps are described below [221], [222], [248], [253]:

This work elicits individual cognitive maps and aggregates them, rather than to use group modeling. The approach is chosen because it gives individuals the time to think through their own mental models and challenge them, without the pressure of time and group dynamics. Elicitation can occur in a workshop setting, in which each of the participants initially works independently to represent their mental models. It can also occur in real-life or computer-facilitated one-on-one cognitive mapping sessions during which a facilitator helps participants through the process. In either case, elicitation step kicks off with an initial introduction of FCM modeling. Then PDF index cards are 
distributed to the participants, who are asked to review titles and descriptions and to add cards with concepts that they deem important but that are missing from the initial set of cards. After this knowledge activation step, participants are asked to identify causal connections between concepts: In order to build Tech Map, each participant, who is a product design-related stakeholders, assesses how technology alternatives ${ }^{9}$, desirable features or functions of the target product are causally connected based on his/her mental model. Borrowing the fundamental structure of an FCM described above (see Figure 4.6), these PDFs correspond to nodes of an FCM while the causal relationships among PDFs are represented as interconnections (or edges). The strength of the causal impact between two factors is represented as a weight in the interval $[-1,1]$. The value of each weight is determined by translation from a linguistic judgment (e.g. "little low," "extremely high," or "medium") to a corresponding numerical value based on If-Then rules. If a contributor adds a new PDF to his/her map that was previously not included in the stack of index cards, he/she is asked to add its detailed description, so that the meaning becomes clear. In later steps, this makes it possible to communicate the map content to other PD team members and to, where appropriate, aggregate new concepts that were added to different individual maps.

After all participants complete their individual maps, the facilitator collects the individual maps. Tech Map is developed by combining the collected individual maps as depicted in Figure 4.9. In a lot of cases, this step will only be possible after the participantgenerated maps are reviewed and cleaned of nodes that are not an objective of the model

\footnotetext{
${ }^{9}$ a technological element (e.g. a component, process, part, etc.) that makes it possible to
} implement features or functions of a product 
despite only having in-arrows, of nodes that are purely definitional, and of nodes that overlap with or duplicate other nodes [248]. After aggregation, this "clean up" may have to be done again with an additional focus on missing connections or unconnected nodes [254].

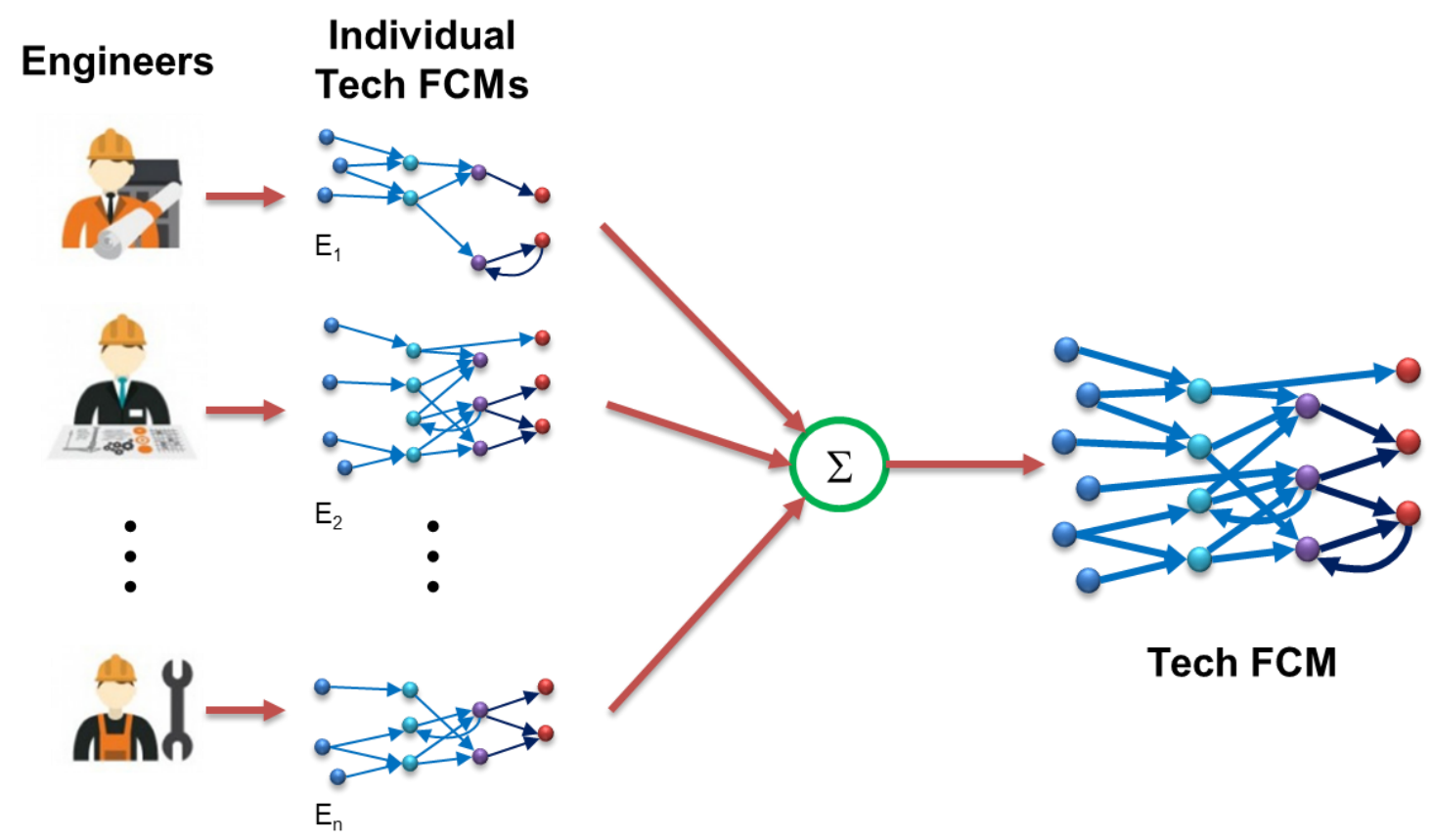

Figure 4.9 Integration of individual maps into a Tech Map

The process for Need Map is similar: Market-oriented stakeholders need to develop a Need Map considering the relationships between required or desirable technical requirements (features or functions), customer needs (benefits) and the customer preference for a target product. The relationships between benefits and customer preference can be weighted in the same way in which relationships in a Tech Map are weighted. However, unlike a Tech Map, a Need Map might include non-technical factors which are difficult to measure quantitatively. 
After the integrated map has been generated and reviewed by an FCM modeler, the resulting model is presented to the contributors to the model and, where appropriate, to additional experts from engineering and marketing division. This occurs in a workshop setting during which the structure and the behavior of the model are discussed. The process is likely iterative until an agreement is reached and, accordingly, time for iterations needs to be planned. Strategies for model review and validation are discussed in section 4.3.

\subsubsection{Model Synthesis (Step 3)}

After the modeling workshops, the two Maps, Tech and Need Maps, need to be integrated to build an aggregated FCM, so-called Product Design Factor Map (PDF Map), which is the core model of the CDRM, as depicted in Figure 4.7. It includes all causal relationships from technology alternatives to customer preference, as depicted in Figure 4.6.

In integrating the two maps, the PDF Map, including the union of the causal nodes in Tech and Need Maps. Then, the adjacency matrix of the Product Factor Map is formed by:

$$
E_{a g g}=E_{\text {tech }}+E_{\text {need }}
$$

Furthermore, as described in Section 4.1.2, each weight of the adjacency matrix

$E_{a g g}$ needs to be calculated considering overlapped nodes and edges between Tech and Need Maps. The resulting PDF Map needs to be tested and validated, as discussed in Section 4.3. 


\subsubsection{Scenario Building (Step 4)}

In this work, any combination of input variables is considered a scenario. FCM computation, which determines what new stable state the system reaches in response to an input scenario, is considered a simulation run. Scenario building thus refers to identifying feasible combinations of input variables that are of interest to the PD team and provide the basis for simulations.

The purpose of the PDF Map is to answer "what if" questions about product concepts, such as "what happens to customer benefit A, if we chose product concept Y, which means increasing the value of technology alternative A, decreasing $\mathrm{B}$, and not implementing C?" To build scenarios, PD engineers need to determine which product concepts or variations of product concepts they want to assess with the help of the simulation model. Concept generation is outside of the scope of this research: it occurs with commonly used product development methods, such as the concept classification tree and the concept combination table are a useful tool [3]. Additionally, the development of simple prototypes with rapid prototyping equipment (e.g., 3D printers) and computer-aided design (CAD) can lead to ideas for modifications of concepts. Regardless of where the ideas for testable product concepts come from, they need to be represented as an initial state vector for the subsequent FCM simulation.

\subsubsection{Simulation (Step 5)}

Prior to simulating scenarios, it is necessary to select appropriate squashing functions and termination conditions with which the output of each FCM calculation reaches to be stable or within a limit cycle, making the behavior of the PDF map close to the real-world [3], [9], [19]. In the CDRM, a hyperbolic tangent function of which the 
lambda is two in Equation (6) is used as the squashing function because it is suitable for qualitative and quantitative problems where it is required to represent degree of increase, a degree of decrease, or stability of a concept according to Tsadiras [231].

Using the PDF map, the value of customer preference (CPV) for each input scenario is calculated. This will be done following the fundamentals of FCM computation described in Section 4.1.1. For computation of the PDF map, the PD team utilizes some specific software (e.g. Mental Modeler [255], spreadsheet software, or packages in

programing languages [256], [257]) enabling to calculate each input scenario and visualize the result of each calculation to support decisions.

Lastly, all of the simulation results need to be reviewed by experienced and experts to validate that the results are reasonable to explain the response of the real-world customer to scenarios.

\subsubsection{Result Analysis and Interpretation (Step 6)}

The project team needs to analyze the results of the FCM simulation. The outputs of the simulation runs are numerical values for $\mathrm{CPV}$ at a point at which the FCM has reached a new stable state. Depending on the choice of squashing function, they fall into the range of -1 and 1 , or 0 and 1 . A value close to 1 indicates high customer value, whereas values around zero or negative values indicate that the product concept has little or no value to the customer. Naturally, these values mean little in absolute terms, but they can be used to compare alternative product concepts against each other and to investigate how changes to a product concept (e.g., the implementation of a technology alternative) impact 
$\mathrm{CPV}$. The scenario that delivers the highest CPV represents the best product concept among the simulated alternative and should, therefore, be selected, all things being equal.

CDRM does not model operational aspects (e.g., feasibility, costs, manufacturability, etc.) of the development project. Consideration of these factors may cause the best product concept (from a customer value perspective) to be nevertheless not selected. However, CDRM shows how the selected (more feasible, cost-effective, easyto-manufacture, etc.) concept fairs with regard to the benefits it delivers in comparison to other concepts. This can be used to identify high leverage concept modifications that can improve the overall CPV.

Moreover, it is quite common that selected product concepts need to be revised and modified during later stages of product development or even after product launch, because new data leads to more insights or because there are market or technological changes [3], [22], [258]-[260]. CDRM can be used to plan such modifications by showing their impact on CPV. Such an analysis requires that new scenarios that represent the proposed modifications are created and simulated (steps 4 and 5). Sometimes this may also require a modification of the PDF Map, e.g., by adding or eliminating concepts and changing weights on the relationships (step 3).

\subsection{Validation of CDRM}

When CDRM is applied to a product development project, it is critical that its FCM models adequately represent the realities of the project. Otherwise, CDRM will lead to poor concept development decisions. When developing the CDRM, and in order to 
answer research questions $1-4^{10}, \mathrm{I}$, therefore, had to consider strategies for ensuring model quality or validity in the context of this research. In order to answer research question $5^{11}$, I additionally have to develop research strategies to evaluate the impact of CDRM on the cognitive distance among product development team members. This evaluation, which aims at the evaluation of the efficacy of CDRM as a method, is not part of the CDRM process: when applying CDRM, practitioners do not need to monitor their cognitive distance. Consequently, I will describe strategies for the evaluation of CDRM as a method in the subsequent chapter.

A model represents the real world in a simplified way, based on the modeler's understanding [211], [217]. As described in prior chapters, the core model of the CDRM consists of two Maps, Tech and Need Maps, which are integrated into a holistic model. Each Map represents the causal relationships between PDFs and customer preference based on the perceptions of the participants and the consensus reached in a group modeling session. The models thus contain a quantitative aggregation of the consensus judgment of the participants about causal relationships and the weight of each relationship. Many different factors can cause this aggregated model to poorly reflect real-world PDFs and their interdependencies. For example, market research can be flawed, team members can choose to not share their knowledge (e.g. because of group dynamics or communication problems), or team knowledge can be misrepresented by the model

\footnotetext{
10 (1) How can the cognition of market-oriented stakeholders and product development engineers be modeled?; (2) How can the cognitive models of market-oriented stakeholders and of PD engineers be integrated to represent the product concept holistically?;(3) How can alternative product concepts (i.e. combinations of design characteristics) be represented in the model as alternative input scenarios? (4) How can the outcomes of alternative input scenarios be used to determine the best product concept alternative? ${ }^{11}$ Does CDRM result in an improved and shared understanding of product design factors among product development team members?
} 
because meanings of PDFs are distorted, modeling errors occur, or because of the limitations of FCM as the modeling language. These and similar threats to model quality/validity are discussed in both quantitative and qualitative research.

For validation of CDRM models, the quantitatively oriented research streams of requirements engineering and systems modeling (particularly with FCM) are of particular importance: The discipline of requirements engineering is concerned with eliciting, modeling, analyzing, and communicating requirements for technical systems, such as software. Requirements engineering uses conceptual models - visual models that show concepts and their relationships - to represent requirements and designs. The discipline models (typically graph structures) and elicitation techniques (among others, cognitive mapping) [261] share a lot of similarities with FCM, and many of the strategies for model validation apply. In particular, requirements engineering emphasizes the importance of ensuring model quality through a rigorous process that lightens cognitive loads, improves readability/traceability, communicates modeling steps and asks stakeholders to confirm that they are correct, and visualizes knowledge to reveal errors in domain knowledge [261]. The use of cognitive mapping and the organization and facilitation of modeling workshops can ensure that these criteria are met by the CDRM process.

Systems Modeling with FCM, on the other hand, is one of several quantitative system modeling techniques that are used to create computational models of complex real-world systems for the purpose of simulation. In particular, it emphasizes the importance of accuracy (i.e., that the quantitative system model is an adequate representation of the real-world system) and sensitivity (i.e., that the quantitative system 
model responds with a sufficient degree of sensitivity to input changes to allow meaningful conclusions). Moreover, Coyle and Exelby summarized a broad measure of agreement in literature dealing with the validation of system modeling as followings [262, pp. 35-36]:

- There is no such thing as absolute validity, only a degree of confidence, which becomes greater as more and more tests are passed.

- The validity of a model can only be assessed in relation to its purpose; it must be capable of answering the questions its sponsor wishes to ask.

- The boundary between what has been included and what has been omitted is crucial.

- The boundary must be small enough to be tractable yet large enough to encompass the solution to the problem.

- Validation by defense of the detail is essential because there are limitless opportunities for making mistakes, especially as there is often a paucity of theory to guide the analysis.

- The behavior of the model must be like that of the real system and changes to the model's structure, parameters and policies must produce changed behavior which is plausible and explicable; this must still be the case even when the changes are extreme.

Considering the above agreement in validating a model, systems modeling with FCM proposes a series of tests about model structure and behavior as described in Table 4.1 [213], [217], [253], [263]. The same tests can be used to ensure the quality of the CDRM models. 
Table 4.1 Applicable tests for validation of PDF map

\begin{tabular}{|l|l|l|}
\hline Type of Test & Name of Test & Description \\
\hline $\begin{array}{l}\text { Model } \\
\text { Structure }\end{array}$ & Structure-Verification Test & $\begin{array}{l}\text { a comparison between the model } \\
\text { structure and the structure of the real- } \\
\text { world problem }\end{array}$ \\
\cline { 2 - 3 } & Parameter-Verification Test & $\begin{array}{l}\text { a comparison between the conceptual } \\
\text { model parameters to the knowledge of } \\
\text { the real system }\end{array}$ \\
\cline { 2 - 3 } $\begin{array}{l}\text { Model } \\
\text { Behavior }\end{array}$ & $\begin{array}{l}\text { Boundary Adequacy Test } \\
\text { Test }\end{array}$ & $\begin{array}{l}\text { a test of whether the model includes all } \\
\text { relevant structure }\end{array}$ \\
\cline { 2 - 3 } & Extreme-Conditions Test & $\begin{array}{l}\text { an examination of how well model- } \\
\text { generated behavior matches observed } \\
\text { behavior of the real system }\end{array}$ \\
\cline { 2 - 3 } & Sensitivity Analysis & $\begin{array}{l}\text { a test if extreme parameter values lead to } \\
\text { rational behavior }\end{array}$ \\
\hline $\begin{array}{l}\text { an identification of sensitive parameters; } \\
\text { an analysis of how changes in some } \\
\text { anameters within plausible range impact } \\
\text { on the others }\end{array}$ \\
\hline
\end{tabular}

Furthermore, Luna-Reyes and Andersen argue that qualitative data, as a main source of information, and their analysis are fundamental for system modeling as well as quantitative data [264]. Particularly, until developing Tech and Need Maps in CDRM, most elements of maps are elicited through qualitative methods, such as content analysis, interviews, and workshops. Accordingly, it is necessary to establish valid qualitative data. Tracy proposes eight criteria of quality in qualitative research: five that apply to the research project in general, namely worthy topic, sincerity, resonance, significant contribution, ethical and meaningful coherence and three that directly relate to the process of collecting and analyzing qualitative data, such as mental models [265]. They are: rich rigor, sincerity, and credibility (see Table 4.2). 


\section{Table 4.2 Selective criteria for excellent qualitative research}

\begin{tabular}{ll}
\hline Criteria for quality & $\begin{array}{l}\text { Various means, practices, and methods through which to } \\
\text { achieve }\end{array}$ \\
\hline & The study uses sufficient, abundant, appropriate, and complex \\
- Theoretical constructs \\
- Data and time in the field \\
- Sample(s) \\
- Data collection and analysis process \\
Rich rigor \\
The study is characterized by \\
- Self-reflexivity about subject values, biases, and inclinations of \\
the researcher(s) \\
- Transparency about the methods and challenges \\
The research is marked by \\
- Thick description, concrete detail, explication of tacit (non- \\
textual) knowledge, and showing rather than telling \\
- Triangulation or crystallization \\
- Multivocality \\
- Member reflections
\end{tabular}

(Source:[171, pp. 840])

Building on these foundations, facilitators and modelers using the CDRM method should review their process choices and results through questions such as, but not limited to:

- Is the configuration of participants reasonable?

- If customers participate as external participants, are they qualified?

- Are engineers and marketing people as internal participants involved in the target product development project directly?

Combining the research on model validation and qualitative research, discussed above, hightlights the importancing of rigor, sincerity, credibility, accuracy, and sensitivity. Taken together, they can be documented in a checklist ensuring CDRM validity, which is depicted in Table 4.3. The checklist is intended for use by practitioners, who use CDRM in their PD projects. 
Table 4.3 Model Validation Checklist

\begin{tabular}{|c|c|c|c|c|c|c|c|c|}
\hline \multirow{3}{*}{ 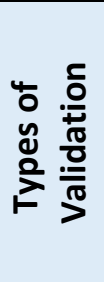 } & \multirow[b]{3}{*}{ Questions } & \multicolumn{5}{|c|}{ Quality } & \multirow[b]{3}{*}{$\begin{array}{l}\text { CHECK } \\
\text { Yes/No }\end{array}$} & \multirow[b]{3}{*}{ NOTE } \\
\hline & & \multicolumn{3}{|c|}{ Qualitative } & \multicolumn{2}{|c|}{ Quantitative } & & \\
\hline & & 꼼. & 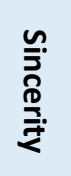 & 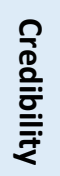 & 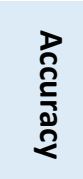 & 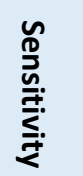 & & \\
\hline \multirow{8}{*}{ 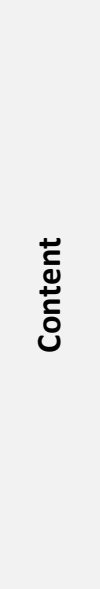 } & $\begin{array}{l}\text { Is the configuration of participants reasonable? If customers participate as external } \\
\text { participants, are they qualified? Are engineers and marketing people as internal } \\
\text { participants involved in the target product development project directly? }\end{array}$ & $\checkmark$ & & & & & & \\
\hline & Are participants well-acquainted with the objective of this research? & $\checkmark$ & & & & & & \\
\hline & $\begin{array}{l}\text { Are participants fully aware of the nature and the procedure of the fuzzy cognitive } \\
\text { mapping technique? Or, is there an expert who can lead and help the participants in } \\
\text { building a model? }\end{array}$ & $\checkmark$ & & & & & & \\
\hline & Are the modeling session well-prepared? & $\checkmark$ & & & & & & \\
\hline & Are the modeling session well-supported (time and resource)? & $\checkmark$ & & & & & & \\
\hline & Are "start concepts" reasonably selected and provided to participants? & $\checkmark$ & & & & & & \\
\hline & Do all participants fully understand the meaning of concepts in a model? & & $\checkmark$ & & & & & \\
\hline & Are the modeling session fully recorded? & & $\checkmark$ & $\checkmark$ & & & & \\
\hline \multirow{4}{*}{ 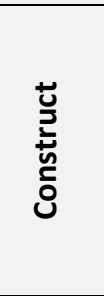 } & Are each relationship and weight assigned reasonably? & & $\checkmark$ & & & & & \\
\hline & $\begin{array}{l}\text { Is the model confirmed after built by participants? Is the model reviewed by } \\
\text { experts? }\end{array}$ & & $\checkmark$ & & & & & \\
\hline & $\begin{array}{l}\text { Before forming a synthesized model, is each map explained to the team? Do the } \\
\text { team approve each model? }\end{array}$ & & & $\boldsymbol{V}$ & & & & \\
\hline & After a synthesized model built, is the model approved by the team? & & & $\boldsymbol{V}$ & & & & \\
\hline \multirow{2}{*}{$\sum_{\Sigma}^{2}$} & Are all elements of the model, relationships and their weights, accurately coded? & & & & $\boldsymbol{V}$ & & & \\
\hline & Is it checked how sensitive the model is about changes in weights? & & & & & $\boldsymbol{V}$ & & \\
\hline
\end{tabular}




\section{RESEARCH IMPLEMENTATION OVERVIEW}

The following chapters describe the implementation and evaluation of the CDRM in the specific context of a home floor cleaning robot for a consumer market. (The rationale for choosing this product is explained below). It was neither practical nor necessary to implement CDRM end-to-end and evaluate all of its steps. For example, the first steps of CDRM - market research and technology alternative exploration - are vital to the success of any new product, need to be done well, and pose ongoing research challenges in product innovation research. However, these challenges are in no way specific to CDRM and do not relate to any of my research questions. Accordingly, I implemented this step only insofar as it was necessary to enable other research steps but did not evaluate it formally. On the other hand, it was very important to understand to what extent CDRM is capable of simulating the outcomes of different product concepts and I researched this question in multiple ways.

CDRM assigns responsibility for the different steps to different actors. Some activities are done by the development team (with the help of a modeling expert or facilitator), others are done by the modeling expert. Throughout this work, I acted as the modeling expert. I furthermore did two studies - characterized as "study 1: feasibility pilot" in this chapter and "study 2: experiment" in the next chapter - to collect data from student participants. These studies approximated how the product development team interacts with CDRM.

Both studies are focused on the same target product, a robotic floor cleaner for home use, which was chosen based on the following considerations: 
First, CDRM is intended for product development projects which have high uncertainty because there are many design factors that are vague, subjective, or difficult to interpret and because high architectural complexity creates many dependencies between product design factors. The chosen product must, therefore, not be too simple and should still have room for innovation regarding its feature set. Moreover, CDRM is not well suited for the following cases:

- ...where the importance of technology is relatively lower than marketing efforts, such as advertisement, packaging, and distribution, which engineers can hardly control (e.g., cosmetic, foods, and personal care products).

- ...where aesthetic design elements are fundamental, but the impact of technological factors is insignificant (e.g., fashion and cosmetic).

- ...where the product design factor architecture is too big and extremely complicated. (e.g., integrated product development projects such as aerospace products and ships [271])

- ...where brand image and price of products critically affect customer purchase intension rather than technological factors (e.g., luxury products).

- ...where most product design factors can be measured quantitatively, and are rarely subjective (e.g., semiconductors, internal components of machinery, and fabrication process equipment).

Because my studies involved student participants, the product furthermore had to be sufficiently interesting and relatable to them so that they could contribute. I also had to ensure that the chosen product would not be so familiar that study participants would be unable to abstract from specific products they know and use. This is why I chose a 
robotic vacuum. An additional advantage of this choice is the availability of published literature on customer needs and designs relating to the product. The specific sources used for each study are described together with the study.

Table 5.1 provides an overview of how I have implemented the different steps of the CDRM through the two studies aforementioned. The following four chapters describe the implementation and evaluation of the CDRM. I start by presenting preliminary data from a small scale feasibility study in Chapter 6. This chapter focuses on investigating whether participants' mental models about a product concept can be represented as FCM by modeling them as combinations of product design factors. Subsequently, the next three chapters explain an experimental study to evaluate the feasibility and effectiveness of the proposed CDRM method. Chapter 7 overviews the experiment, the study setting, and the participants, and describes the data collection process for the experiment. Chapter 8 explains two methods to analyze the surveys submitted during the experiment and to compare individual and group FCMs. Lastly, in Chapter 9, the results obtained by the experiment are described, and a comprehensive analysis is provided. 
Table 5.1 Overview of implementation

\begin{tabular}{|c|c|c|c|c|c|}
\hline \multirow[b]{2}{*}{ CDRM Step } & \multirow[b]{2}{*}{ Activity Description } & \multicolumn{2}{|c|}{ Implementation Studies } & \multirow[b]{2}{*}{ Actor(s) } & \multirow[b]{2}{*}{$\begin{array}{l}\text { Research } \\
\text { Question }\end{array}$} \\
\hline & & $\begin{array}{l}\text { Study 1: } \\
\text { Feasibility Pilot } \\
\text { (Ch.6) }\end{array}$ & $\begin{array}{l}\text { Study 2: } \\
\text { Experiment } \\
\text { (Ch. 7-9) }\end{array}$ & & \\
\hline $\begin{array}{l}\text { Market Research \& } \\
\text { Technology Alternative } \\
\text { Exploration }\end{array}$ & $\begin{array}{l}\text { Background research on home floor } \\
\text { cleaning robots based on published } \\
\text { research, and product videos }\end{array}$ & 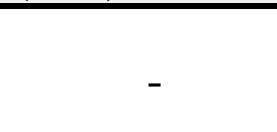 & $\checkmark$ & Researcher & - \\
\hline $\begin{array}{l}\text { Basic Product Design } \\
\text { Factor (PDF) } \\
\text { Elicitation }\end{array}$ & $\begin{array}{l}\text { Identification of PDFs to be considered } \\
\text { in the FCMs }\end{array}$ & $\checkmark$ & $\checkmark$ & $\begin{array}{c}\text { Researcher \& } \\
\text { Participants }\end{array}$ & RQ. 1 \\
\hline $\begin{array}{l}\text { Need-Map } \\
\text { Development }\end{array}$ & $\begin{array}{l}\text { Creation of an FCM model about } \\
\text { customer needs and PDFs }\end{array}$ & $\checkmark$ & $\checkmark$ & $\begin{array}{c}\text { Researcher \& } \\
\text { Participants }\end{array}$ & RQ. 1 \\
\hline $\begin{array}{l}\text { Tech-Map } \\
\text { Development }\end{array}$ & $\begin{array}{l}\text { Creation of an FCM model about PDFs } \\
\text { as technological alternatives }\end{array}$ & $\checkmark$ & $\checkmark$ & $\begin{array}{c}\text { Researcher \& } \\
\text { Participants }\end{array}$ & $\begin{array}{l}\text { RQ. } 1 \text { \& } \\
\text { RQ. } 5\end{array}$ \\
\hline $\begin{array}{l}\text { Model Synthesis } \\
\text { - PDF Map Creation }\end{array}$ & $\begin{array}{l}\text { Integration of Need-Map and Tech-Map } \\
\text { to create a holistic picture of product } \\
\text { concepts, as a PDF map }\end{array}$ & - & $\checkmark$ & Researcher & RQ. 2 \\
\hline Scenario Creation & $\begin{array}{l}\text { Creation of product concept alternatives } \\
\text { expressed as state vectors as scenario } \\
\text { inputs for FCM simulation }\end{array}$ & $\checkmark$ & $\checkmark$ & $\begin{array}{c}\text { Researcher \& } \\
\text { Participants }\end{array}$ & RQ. 3 \\
\hline Simulation & $\begin{array}{l}\text { Calculation of steady-states for a PDF } \\
\text { map with different scenario inputs to } \\
\text { select from product concept alternatives }\end{array}$ & $\checkmark$ & $\checkmark$ & Researcher & RQ. 4 \\
\hline
\end{tabular}


In designing the study, I had to consider several practical limitations: real-world product development projects are generally confidential and not accessible to researchers, in particular in success critical stages, such as concept development. Purely experimental studies, in which the researcher defines project tasks with no bearing on the company's business and no confidentiality concerns, on the other hand, provide little value to companies, and they are therefore unlikely to let their PD teams participate.

This experiential study, therefore, takes place in an educational setting: in higher education, it is increasingly common to train and guide students to develop experience, knowledge, and expertise through real-world learning models that permit students to arm themselves with enough skillsets for more complex and ambiguous real-world projects after their graduations [266]-[268]. Table 5.2 presents the dominant models of real-world learning formats and the main features of each model. Particularly, capstone real-world learning opportunities enables students to experience practical interaction and collaboration in multidisciplinary team settings similar to real-world experiences. 
Table 5.2 Dominant models of real-world learning formats

\begin{tabular}{|c|c|c|c|}
\hline & $\begin{array}{l}\text { Project-based } \\
\text { learning }\end{array}$ & Service-learning & Internship \\
\hline $\begin{array}{l}\text { Outcomes: what } \\
\text { students } \\
\text { learn/benefit }\end{array}$ & $\begin{array}{l}\text { Collaborative } \\
\text { problem-solving } \\
\text { capacity }\end{array}$ & $\begin{array}{l}\text { Education and } \\
\text { teaching capacity }\end{array}$ & $\begin{array}{l}\text { Professional } \\
\text { working experience; } \\
\text { career development }\end{array}$ \\
\hline $\begin{array}{l}\text { Practices: what } \\
\text { students do }\end{array}$ & $\begin{array}{l}\text { Collaborating with } \\
\text { partners to develop } \\
\text { solution } \\
\text { approaches }\end{array}$ & Educating people & $\begin{array}{l}\text { Assisting or } \\
\text { working on a } \\
\text { professional project }\end{array}$ \\
\hline $\begin{array}{l}\text { Interaction with } \\
\text { stakeholder }\end{array}$ & $\begin{array}{l}\text { Two-way } \\
\text { knowledge } \\
\text { generation (co- } \\
\text { production) }\end{array}$ & $\begin{array}{l}\text { One-way } \\
\text { knowledge transfer } \\
\text { (students to } \\
\text { community) }\end{array}$ & $\begin{array}{l}\text { One-way knowledge } \\
\text { transfer (employer } \\
\text { to student and } \\
\text { student to employer) }\end{array}$ \\
\hline $\begin{array}{l}\text { Integration of } \\
\text { theory and } \\
\text { practice }\end{array}$ & $\begin{array}{l}\text { Explicit, supervised } \\
\text { by faculty and } \\
\text { stakeholder }\end{array}$ & $\begin{array}{l}\text { Implicit, not } \\
\text { supervised by } \\
\text { faculty }\end{array}$ & $\begin{array}{l}\text { Implicit, not } \\
\text { supervised by } \\
\text { faculty }\end{array}$ \\
\hline Impacts on world & $\begin{array}{l}\text { Systemic } \\
\text { innovation }\end{array}$ & $\begin{array}{l}\text { Support of social } \\
\text { innovation and } \\
\text { change }\end{array}$ & Modular innovation \\
\hline
\end{tabular}

(adapted from [268, p. 311])

Considering the substantive difficulty of accessing a real-world NPD project, this experiment, therefore, utilized NPD-related engineering projects as an alternative in testing and demonstrating the effectiveness of CDRM. Naturally, there are similarities and differences in NPD practices between projects in engineering education and projects in industry. Table 5.3 presents them, based on a longitudinal study by Cobb et al. that uses interviews with the alumni of a graduate-level NPD [269]. 
Table 5.3 Similarities and differences between NPD practices in education and in industry

\begin{tabular}{|c|c|c|}
\hline & In NPD education & In industry \\
\hline Similarities & \multicolumn{2}{|c|}{$\begin{array}{l}\text { - } \text { Requiring teamwork skills } \\
\text { - Existence of dysfunctional teams } \\
\text { - Unpredictability combined with the amount of work }\end{array}$} \\
\hline Differences & 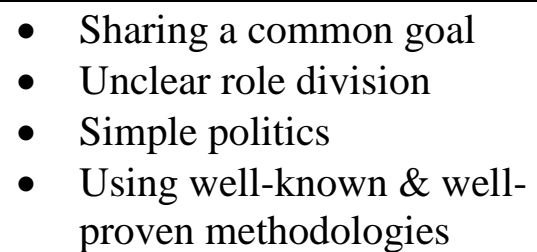 & $\begin{array}{l}\text { - Various stakes } \\
\text { - Clear role division } \\
\text { - Complicated politics } \\
\text { - Using "unfamiliar" } \\
\text { methodologies \& processes }\end{array}$ \\
\hline
\end{tabular}

(Source: adapted from [269])

In contrast to Cobb et al., however, the experimental design uses an unfamiliar methodology and process, namely CDRM, and thus reduces the inherent differences between industry and educational NPD. 


\section{STUDY 1: FEASIBILITY PILOT}

This section summarizes the approach and results of the first study ${ }^{12}$, which was a feasibility pilot of CDRM. (For a more detailed discussion, please refer to the original publication) In this study, I wanted to specifically investigate if participants' mental models about a product concept can be represented as FCM by modeling them as combinations of product design factors.

The product concept for the study was a robotic vacuum cleaner, intended for the consumer market. Two mental models, a Need and a Tech Map were developed by six engineering graduate students (as potential customers of the vacuum cleaner) and by two robotic engineers who have over ten- and seven-year work experiences, respectively. I used a comprehensive literature review of studies on robotic vacuum cleaners, which covered development issues, market trends, and consumer response, to identify initial PDFs and provide them, as start concepts, to both groups. Both groups added new concepts, which were not included in the start concepts, to reflect what they felt contributes to a desirable product. Mental models were developed in two face-to-face meetings (one per group) by assigning causal relationships between initial and participant provided concepts, as well as weights for each relationship. Figure 6.1 is the causal cognitive map, as Tech Map, of designing a robotic vacuum cleaner concept. This map includes fifteen concepts defined in Table 6.1. Moreover, each relationship has a

\footnotetext{
${ }^{12}$ This study was presented and published at Portland International Conference on Management of Engineering and Technology in 2014. (B. S. Yoon and A. J. Jetter, "Investigation of Different Perspectives between Developers and Customers: Robotic Vacuum Cleaners," in Proceedings of PICMET '14 Conference: Portland International Center for Management of Engineering and Technology; Infrastructure and Service Integration, 2014, pp. 2307-2313).
} 
corresponding value assigned through the discussion of experienced robotics engineers and within a range between -1 to 1 , which is shown in the adjacency matrix in Table 6.2.

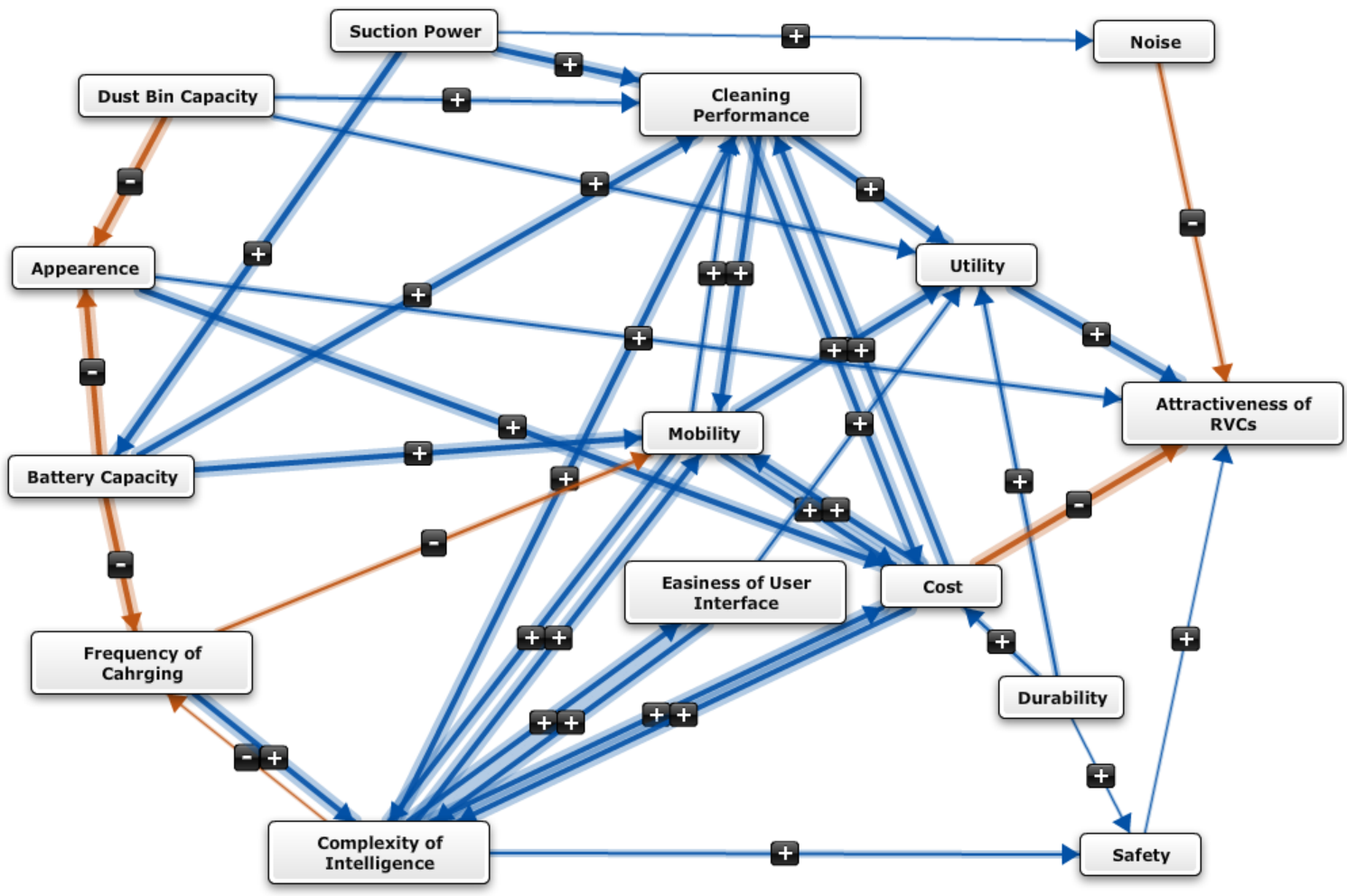

Figure 6.1 Tech Map of a robotic vacuum cleaner design concept 
Table 6.1 Definition of each concept in Tech Map

\begin{tabular}{|c|c|c|}
\hline No. & Concepts & Definition \\
\hline 1 & Attractiveness of RVCs & the degree of attractiveness which stimulate customers to buy an RVC \\
\hline 2 & Utility & the degree of usefulness which makes an RVC convenient for users \\
\hline 3 & Cost & the amount of money that has to be spent on developing an RVC \\
\hline 4 & Safety & the state of not being dangerous or harmful in using an RVC \\
\hline 5 & Noise & the degree of loudness or unpleasant sound in using an RVC \\
\hline 6 & Cleaning Performance & the degree of performance how well an RVC can clean a specific location \\
\hline 7 & Mobility & the degree of capability of moving for cleaning and charging \\
\hline 8 & Easiness of User Interface & $\begin{array}{l}\text { the degree of ease of use in inputting specific commands or recognizing the status of an } \\
\text { RVC }\end{array}$ \\
\hline 9 & Durability & the degree of staying good condition in usual usage of an RVC \\
\hline 10 & Suction Power & the maximum pressure difference that the pump can create for cleaning \\
\hline 11 & Complexity of Intelligence & $\begin{array}{l}\text { the quality or state of being complex in controlling an RVC with artificial intelligence or } \\
\text { control algorithms }\end{array}$ \\
\hline 12 & Frequency of Charging & the number of times that an RVC have to charge its battery to clean a specific area \\
\hline 13 & Battery Capacity & a measure of the charge stored by the battery in an RVC \\
\hline 14 & Appearance & the way that RVC looks \\
\hline 15 & Dust Bin Capacity & the maximum volume where an RVC can store dust or debris \\
\hline
\end{tabular}

$\stackrel{\circ}{\omega}$ 
Table 6.2 The adjacency matrix corresponding to Tech Map

\begin{tabular}{|c|c|c|c|c|c|c|c|c|c|c|c|c|c|c|c|}
\hline & 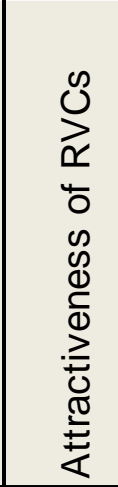 & 売 & $\begin{array}{l}\text { गू } \\
\text { d }\end{array}$ & $\frac{\vec{d}}{\stackrel{\mathbb{N}}{\mathbb{N}}}$ & $\begin{array}{l}0 \\
\frac{\infty}{0} \\
Z\end{array}$ & 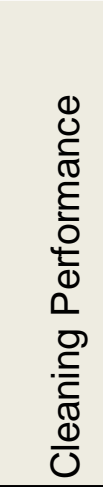 & $\begin{array}{l}\stackrel{\gtrsim}{=} \\
\stackrel{\overline{0}}{0} \\
\sum\end{array}$ & 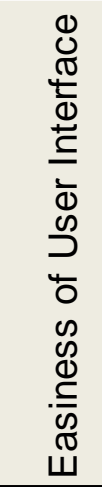 & 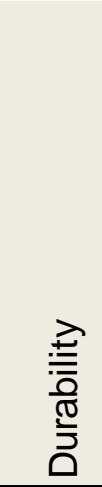 & 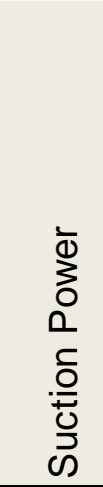 & 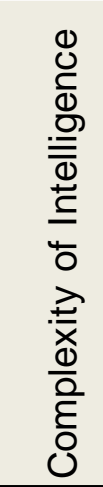 & 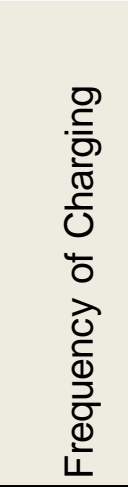 & 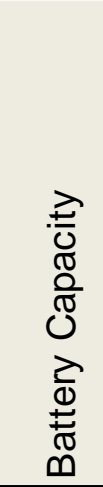 & 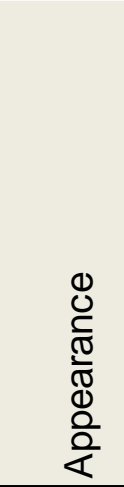 & 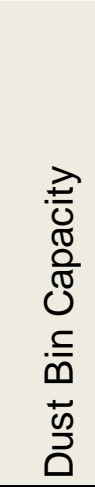 \\
\hline Attractiveness of RVCs & 0.00 & 0.00 & 0.00 & 0.00 & 0.00 & 0.00 & 0.00 & 0.00 & 0.00 & 0.00 & 0.00 & 0.00 & 0.00 & 0.00 & 0.00 \\
\hline Utility & 1.00 & 0.00 & 0.00 & 0.00 & 0.00 & 0.00 & 0.00 & 0.00 & 0.00 & 0.00 & 0.00 & 0.00 & 0.00 & 0.00 & 0.00 \\
\hline Cost & -1.00 & 0.00 & 0.00 & 0.00 & 0.00 & 1.00 & 1.00 & 0.00 & 0.00 & 0.00 & 1.00 & 0.00 & 0.00 & 0.00 & 0.00 \\
\hline Safety & 0.25 & 0.00 & 0.00 & 0.00 & 0.00 & 0.00 & 0.00 & 0.00 & 0.00 & 0.00 & 0.00 & 0.00 & 0.00 & 0.00 & 0.00 \\
\hline Noise & -0.50 & 0.00 & 0.00 & 0.00 & 0.00 & 0.00 & 0.00 & 0.00 & 0.00 & 0.00 & 0.00 & 0.00 & 0.00 & 0.00 & 0.00 \\
\hline Cleaning Performance & 0.00 & 1.00 & 1.00 & 0.00 & 0.00 & 0.00 & 1.00 & 0.00 & 0.00 & 0.00 & 0.00 & 0.00 & 0.00 & 0.00 & 0.00 \\
\hline Mobility & 0.00 & 1.00 & 1.00 & 0.00 & 0.00 & 0.50 & 0.00 & 0.00 & 0.00 & 0.00 & 1.00 & 0.00 & 0.00 & 0.00 & 0.00 \\
\hline Easiness of User Interface & 0.00 & 0.50 & 0.00 & 0.00 & 0.00 & 0.00 & 0.00 & 0.00 & 0.00 & 0.00 & 1.00 & 0.00 & 0.00 & 0.00 & 0.00 \\
\hline Durability & 0.00 & 0.50 & 0.50 & 0.25 & 0.00 & 0.00 & 0.00 & 0.00 & 0.00 & 0.00 & 0.00 & 0.00 & 0.00 & 0.00 & 0.00 \\
\hline Suction Power & 0.00 & 0.00 & 0.00 & 0.00 & 0.25 & 1.00 & 0.00 & 0.00 & 0.00 & 0.00 & 0.00 & 0.00 & 1.00 & 0.00 & 0.00 \\
\hline Complexity of Intelligence & 0.00 & 0.00 & 1.00 & 0.50 & 0.00 & 1.00 & 1.00 & 1.00 & 0.00 & 0.00 & 0.00 & -0.25 & 0.00 & 0.00 & 0.00 \\
\hline Frequency of Charging & 0.00 & 0.00 & 0.00 & 0.00 & 0.00 & 0.00 & -0.50 & 0.00 & 0.00 & 0.00 & 1.00 & 0.00 & 0.00 & 0.00 & 0.00 \\
\hline Battery Capacity & 0.00 & 0.00 & 0.00 & 0.00 & 0.00 & 1.00 & 1.00 & 0.00 & 0.00 & 0.00 & 0.00 & -1.00 & 0.00 & -1.00 & 0.00 \\
\hline Appearance & 0.50 & 0.00 & 1.00 & 0.00 & 0.00 & 0.00 & 0.00 & 0.00 & 0.00 & 0.00 & 0.00 & 0.00 & 0.00 & 0.00 & 0.00 \\
\hline Dust Bin Capacity & 0.00 & 0.50 & 0.00 & 0.00 & 0.00 & 0.50 & 0.00 & 0.00 & 0.00 & 0.00 & 0.00 & 0.00 & 0.00 & -1.00 & 0.00 \\
\hline
\end{tabular}

$\stackrel{\triangleright}{\not}$ 
For Need Map, the graduate students built a conceptual cognitive map, as current and potential customers, and assigned relationships. Figure 6.2 is the causal cognitive map that shows the drivers of product desirability from the perspective of the customers. This map includes the twenty-two concepts defined in Table 6.3. The weight of the causal connections between the concepts in the range between -1 to 1 is represented in the adjacency matrix in Table 6.4 .

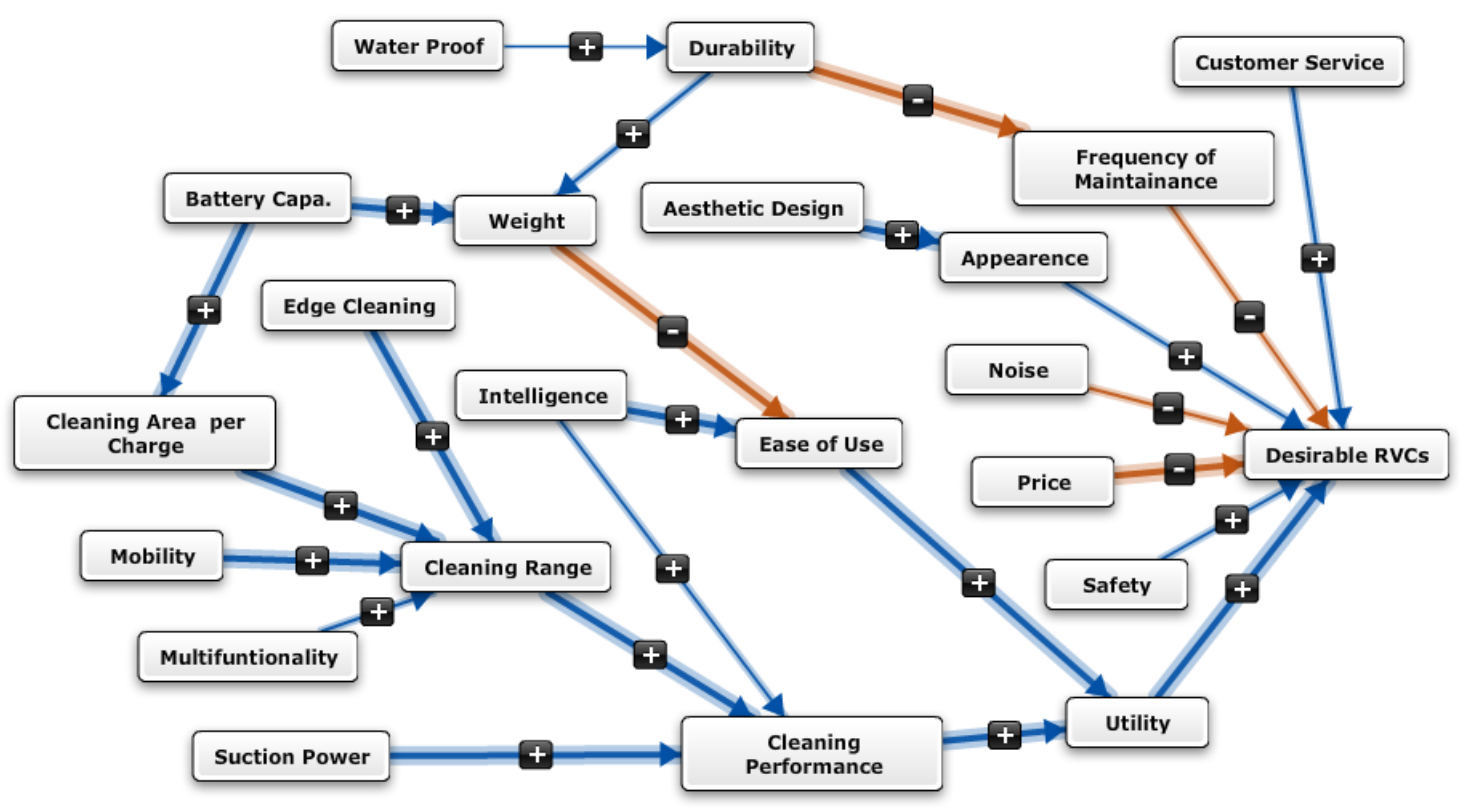

Figure 6.2 Need Map of a robotic vacuum cleaner design concept 
Table 6.3 Definition of each concept in Need Map

\begin{tabular}{|c|c|c|}
\hline No. & Concepts & Definition \\
\hline 1 & Desirable RVCs & the degree of desirability that customers want to buy an RVC \\
\hline 2 & Customer Service & the degree of how much customers satisfy customer service of an RVC \\
\hline 3 & Frequency of Maintenance & the number of times that an RVC needs maintenance for keeping normal condition \\
\hline 4 & Appearance & the external show of an RVC \\
\hline 5 & Noise & the degree of loudness or unpleasant sound in using an RVC \\
\hline 6 & Price & the amount of money that customers pay for an RVC \\
\hline 7 & Safety & the state of not being dangerous or harmful in using an RVC \\
\hline 8 & Utility & the degree of usefulness which makes an RVC convenient for users \\
\hline 9 & Durability & the degree of staying good condition in usual usage of an RVC \\
\hline 10 & Aesthetic Design & the beauty or appeal of an RVC that customers appreciate \\
\hline 11 & Ease of Use & the ability of a customer to readily and successfully perform a task with an RVC \\
\hline 12 & Cleaning Performance & the degree of performance how well an RVC can clean a specific location \\
\hline 13 & Weight & a measurement that indicates how heavy an RVC is \\
\hline 14 & Intelligence & $\begin{array}{l}\text { the ability of an RVC to deal with different situations, based on its artificial intelligence or } \\
\text { programmed algorithms }\end{array}$ \\
\hline 15 & Cleaning Range & the range of area that an RVC can move and clean \\
\hline 16 & Water Proof & a special design to prevent water from entering into the body of an RVC \\
\hline 17 & Battery Capacity & a measure of the charge stored by the battery in an RVC \\
\hline 18 & Edge Cleaning & the ability of an RVC to access edges or corners for cleaning \\
\hline 19 & Cleaning Area per Charge & the area an RVC can clean after charging its battery fully \\
\hline 20 & Mobility & the degree of capability of moving for cleaning and charging \\
\hline 21 & Multifunctionality & the ability of an RVC to clean various floor types and in different modes. \\
\hline 22 & Suction Power & the maximum pressure difference that the pump can create for cleaning \\
\hline
\end{tabular}


Table 6.4 The adjacency matrix corresponding to Need Map

\begin{tabular}{|c|c|c|c|c|c|c|c|c|c|c|c|c|c|c|c|c|c|c|c|c|c|c|}
\hline & 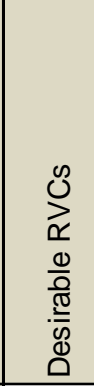 & 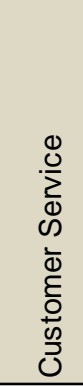 & 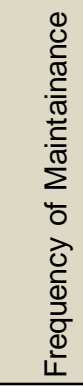 & $\begin{array}{l}0 \\
0 \\
\frac{0}{0} \\
\frac{0}{\pi} \\
\searrow \\
\frac{0}{2} \\
\frac{0}{4}\end{array}$ & $\begin{array}{l}0 \\
\frac{\infty}{0} \\
2\end{array}$ & $\begin{array}{l}0 \\
.00 \\
0.0\end{array}$ & 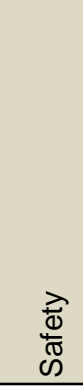 & $\stackrel{\gtrsim}{\stackrel{\nexists}{J}}$ & 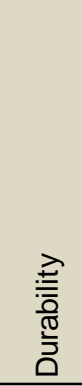 & 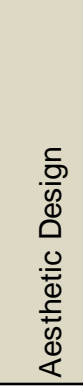 & 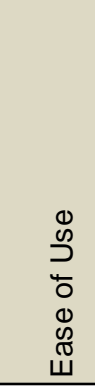 & 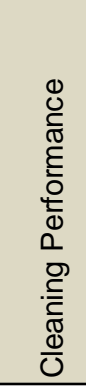 & $\begin{array}{l}\frac{t}{0} \\
\frac{0}{0} \\
3\end{array}$ & 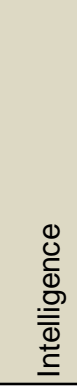 & 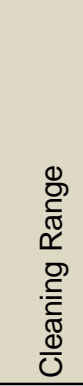 & 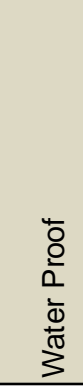 & 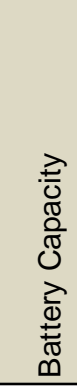 & 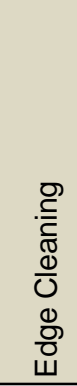 & 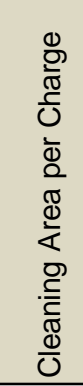 & 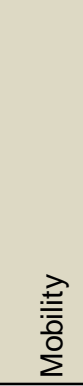 & 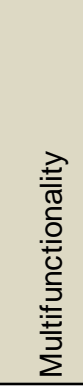 & 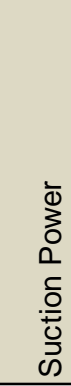 \\
\hline Desirable RVCs & 0.00 & 0.00 & 0.00 & 0.00 & 0.00 & 0.00 & 0.00 & 0.00 & 0.00 & 0.00 & 0.00 & 0.00 & 0.00 & 0.00 & 0.00 & 0.00 & 0.00 & 0.00 & 0.00 & 0.00 & 0.00 & 0.00 \\
\hline Customer Service & 0.50 & 0.00 & 0.00 & 0.00 & 0.00 & 0.00 & 0.00 & 0.00 & 0.00 & 0.00 & 0.00 & 0.00 & 0.00 & 0.00 & 0.00 & 0.00 & 0.00 & 0.00 & 0.00 & 0.00 & 0.00 & 0.00 \\
\hline Frequency of Maintainance & -0.50 & 0.00 & 0.00 & 0.00 & 0.00 & 0.00 & 0.00 & 0.00 & 0.00 & 0.00 & 0.00 & 0.00 & 0.00 & 0.00 & 0.00 & 0.00 & 0.00 & 0.00 & 0.00 & 0.00 & 0.00 & 0.00 \\
\hline Appearance & 0.50 & 0.00 & 0.00 & 0.00 & 0.00 & 0.00 & 0.00 & 0.00 & 0.00 & 0.00 & 0.00 & 0.00 & 0.00 & 0.00 & 0.00 & 0.00 & 0.00 & 0.00 & 0.00 & 0.00 & 0.00 & 0.00 \\
\hline Noise & -0.50 & 0.00 & 0.00 & 0.00 & 0.00 & 0.00 & 0.00 & 0.00 & 0.00 & 0.00 & 0.00 & 0.00 & 0.00 & 0.00 & 0.00 & 0.00 & 0.00 & 0.00 & 0.00 & 0.00 & 0.00 & 0.00 \\
\hline Price & -1.00 & 0.00 & 0.00 & 0.00 & 0.00 & 0.00 & 0.00 & 0.00 & 0.00 & 0.00 & 0.00 & 0.00 & 0.00 & 0.00 & 0.00 & 0.00 & 0.00 & 0.00 & 0.00 & 0.00 & 0.00 & 0.00 \\
\hline Safety & 0.50 & 0.00 & 0.00 & 0.00 & 0.00 & 0.00 & 0.00 & 0.00 & 0.00 & 0.00 & 0.00 & 0.00 & 0.00 & 0.00 & 0.00 & 0.00 & 0.00 & 0.00 & 0.00 & 0.00 & 0.00 & 0.00 \\
\hline Utility & 1.00 & 0.00 & 0.00 & 0.00 & 0.00 & 0.00 & 0.00 & 0.00 & 0.00 & 0.00 & 0.00 & 0.00 & 0.00 & 0.00 & 0.00 & 0.00 & 0.00 & 0.00 & 0.00 & 0.00 & 0.00 & 0.00 \\
\hline Durability & 0.00 & 0.00 & -1.00 & 0.00 & 0.00 & 0.00 & 0.00 & 0.00 & 0.00 & 0.00 & 0.00 & 0.00 & 0.50 & 0.00 & 0.00 & 0.00 & 0.00 & 0.00 & 0.00 & 0.00 & 0.00 & 0.00 \\
\hline Aesthetic Design & 0.00 & 0.00 & 0.00 & 1.00 & 0.00 & 0.00 & 0.00 & 0.00 & 0.00 & 0.00 & 0.00 & 0.00 & 0.00 & 0.00 & 0.00 & 0.00 & 0.00 & 0.00 & 0.00 & 0.00 & 0.00 & 0.00 \\
\hline Ease of Use & 0.00 & 0.00 & 0.00 & 0.00 & 0.00 & 0.00 & 0.00 & 1.00 & 0.00 & 0.00 & 0.00 & 0.00 & 0.00 & 0.00 & 0.00 & 0.00 & 0.00 & 0.00 & 0.00 & 0.00 & 0.00 & 0.00 \\
\hline Cleaning Performance & 0.00 & 0.00 & 0.00 & 0.00 & 0.00 & 0.00 & 0.00 & 1.00 & 0.00 & 0.00 & 0.00 & 0.00 & 0.00 & 0.00 & 0.00 & 0.00 & 0.00 & 0.00 & 0.00 & 0.00 & 0.00 & 0.00 \\
\hline Weight & 0.00 & 0.00 & 0.00 & 0.00 & 0.00 & 0.00 & 0.00 & 0.00 & 0.00 & 0.00 & -1.00 & 0.00 & 0.00 & 0.00 & 0.00 & 0.00 & 0.00 & 0.00 & 0.00 & 0.00 & 0.00 & 0.00 \\
\hline Intelligence & 0.00 & 0.00 & 0.00 & 0.00 & 0.00 & 0.00 & 0.00 & 0.00 & 0.00 & 0.00 & 1.00 & 0.50 & 0.00 & 0.00 & 0.00 & 0.00 & 0.00 & 0.00 & 0.00 & 0.00 & 0.00 & 0.00 \\
\hline Cleaning Range & 0.00 & 0.00 & 0.00 & 0.00 & 0.00 & 0.00 & 0.00 & 0.00 & 0.00 & 0.00 & 0.00 & 1.00 & 0.00 & 0.00 & 0.00 & 0.00 & 0.00 & 0.00 & 0.00 & 0.00 & 0.00 & 0.00 \\
\hline Water Proof & 0.00 & 0.00 & 0.00 & 0.00 & 0.00 & 0.00 & 0.00 & 0.00 & 0.25 & 0.00 & 0.00 & 0.00 & 0.00 & 0.00 & 0.00 & 0.00 & 0.00 & 0.00 & 0.00 & 0.00 & 0.00 & 0.00 \\
\hline Battery Capacity & 0.00 & 0.00 & 0.00 & 0.00 & 0.00 & 0.00 & 0.00 & 0.00 & 0.00 & 0.00 & 0.00 & 0.00 & 1.00 & 0.00 & 0.00 & 0.00 & 0.00 & 0.00 & 1.00 & 0.00 & 0.00 & 0.00 \\
\hline Edge Cleaning & 0.00 & 0.00 & 0.00 & 0.00 & 0.00 & 0.00 & 0.00 & 0.00 & 0.00 & 0.00 & 0.00 & 0.00 & 0.00 & 0.00 & 1.00 & 0.00 & 0.00 & 0.00 & 0.00 & 0.00 & 0.00 & 0.00 \\
\hline Cleaning Area per Charge & 0.00 & 0.00 & 0.00 & 0.00 & 0.00 & 0.00 & 0.00 & 0.00 & 0.00 & 0.00 & 0.00 & 0.00 & 0.00 & 0.00 & 1.00 & 0.00 & 0.00 & 0.00 & 0.00 & 0.00 & 0.00 & 0.00 \\
\hline Mobility & 0.00 & 0.00 & 0.00 & 0.00 & 0.00 & 0.00 & 0.00 & 0.00 & 0.00 & 0.00 & 0.00 & 0.00 & 0.00 & 0.00 & 1.00 & 0.00 & 0.00 & 0.00 & 0.00 & 0.00 & 0.00 & 0.00 \\
\hline & 0.00 & 0.00 & 0.00 & 0.00 & 0.00 & 0.00 & 0.00 & 0.00 & 0.00 & 0.00 & 0.00 & 0.00 & 0.00 & 0.00 & 0.50 & 0.00 & 0.00 & 0.00 & 0.00 & 0.00 & 0.00 & 0.00 \\
\hline Suction Power & 0.00 & 0.00 & 0.00 & 0.00 & 0.00 & 0.00 & 0.00 & 0.00 & 0.00 & 0.00 & 0.00 & 1.00 & 0.00 & 0.00 & 0.00 & 0.00 & 0.00 & 0.00 & 0.00 & 0.00 & 0.00 & 0.00 \\
\hline
\end{tabular}


In comparing the two causal cognitive maps, Tech Map shows a more complicated structure but includes fewer concepts than Need Map. In particular, in Tech Map, there are several relationships between concepts that impact product attractiveness, such as between cleaning performance and cost, between cleaning performance and mobility, between mobility and cost, between mobility and complexity of intelligence, and between complexity of intelligence and cost. Also, there are some feedback loops. For example, increase of mobility impacts the complexity of intelligence. If complexity of intelligence increases, frequency of charging will be decreased and thus affect mobility.

Need Map, on the other hand, is relatively simple and shows very few interdependencies between the concepts that are connected to the desirability of RVCs, such as price, noise, safety and customer service. This (wrongly) implies that they can be improved without trading off any other product characteristic, such as an increase in price. Lastly, in Need Map, there are no feedback loops.

The two causal cognitive maps share common concepts such as durability, suction power and battery capacity, which are only to a small extent influenced by other concepts. They are identified as product design factors. These design factors are used to investigate how the two goal concepts, "attractiveness of RVCs" and "desirable RVCs," are affected when one or several design factors change. The change is modeled as an activation of the respective concept in the initial state vector. 
Table 6.5 Scenario of each case and result value

\begin{tabular}{c|ccc|cc}
\hline \multirow{2}{*}{$\begin{array}{c}\text { Case } \\
\text { No. }\end{array}$} & \multicolumn{2}{|c|}{ Activated Design Factors } & \multicolumn{2}{c}{ Settled Value of Each Goal Concept } \\
\cline { 2 - 6 } & Durability & $\begin{array}{c}\text { Suction } \\
\text { Power }\end{array}$ & $\begin{array}{c}\text { Battery } \\
\text { Capacity }\end{array}$ & $\begin{array}{c}\text { Attractiveness of RVCs } \\
\text { in Tech Map }\end{array}$ & $\begin{array}{c}\text { Desirable RVCs } \\
\text { in Need Map }\end{array}$ \\
\hline 1 & 1 & 0 & 0 & 0.42 & -0.70 \\
2 & 0 & 1 & 0 & -0.78 & 0.96 \\
3 & 0 & 0 & 1 & -0.52 & 0.00 \\
4 & 1 & 1 & 0 & -0.75 & 0.83 \\
5 & 1 & 0 & 1 & -0.47 & 0.74 \\
6 & 0 & 1 & 1 & -0.78 & 0.16 \\
7 & 1 & 1 & 1 & -0.75 & 0.80 \\
\hline
\end{tabular}

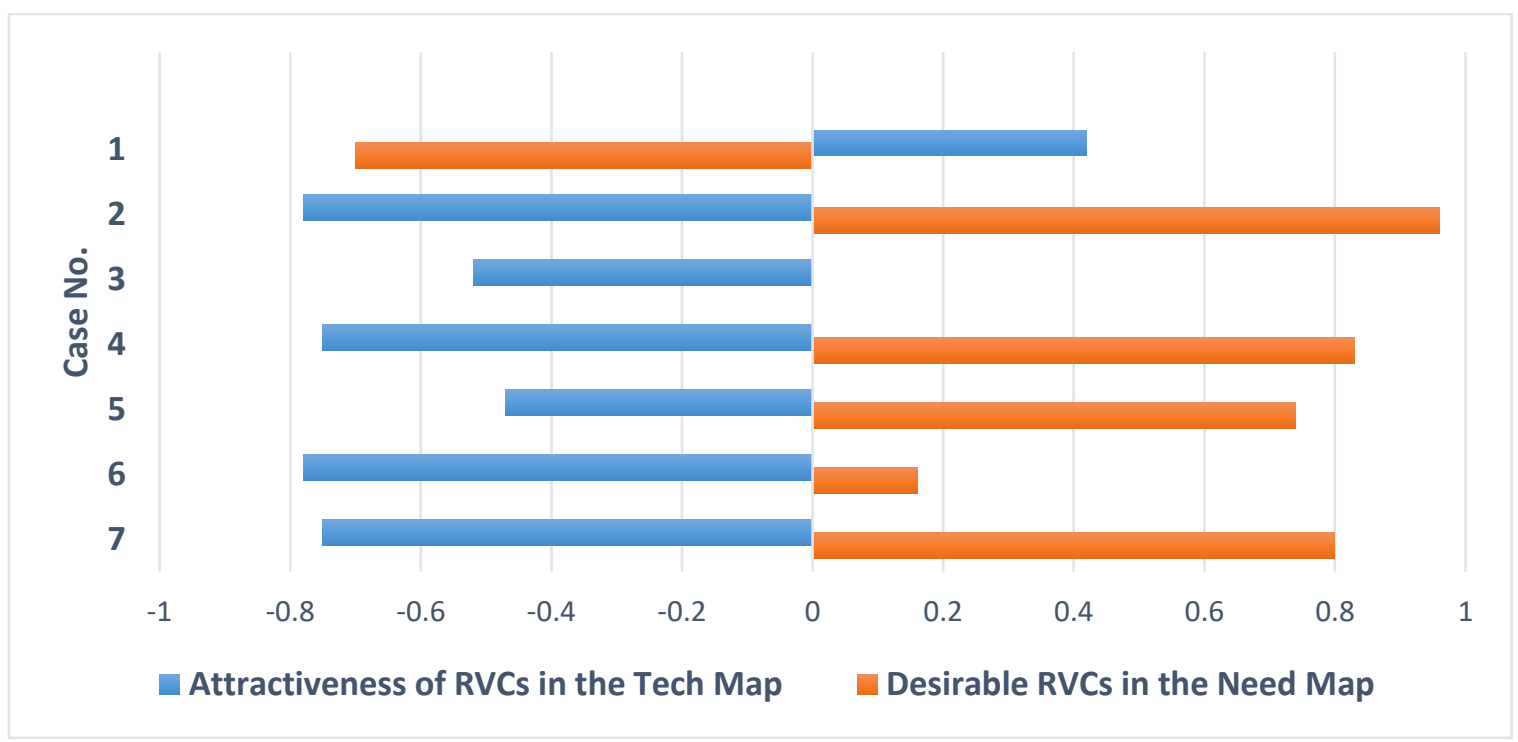

Figure 6.3 Settled value of each goal concept

Table 6.5 shows the final states of the two goal concepts affected by activation of the design factors in both models with a hyperbolic tangent as squashing function. Figure 6.3 graphically represents the final states.

The "Attractiveness of RVCs" concept in Tech Map is negatively affected by activated design parameters in all cases except in case 1 in which only durability is activated. With the exception of durability, the engineers perceive these changes to ultimately lead to a decrease in product attractiveness. The same changes, however, have positive or at least neutral impacts on the "Desirable RVCs" concept in Need Map, 
implying that the customers would welcome the changes which the engineers reject. The activation of the durability parameter in case 1 , which positively influences the "Attractiveness of RVCs" concept from the perspective of the engineers, actually negatively influences the "Desirable RVCs" concept. However, the impact of this case is relatively weaker than in other cases. It implies that the durability of RVCs, by itself, is a less important concept for increasing attractiveness and preference of RVCs in both views. Also, activating only the concept of battery capacity (case 3 ) shows negative effects in the perspective of the engineers while it has no influence in the Need Map. In other words, battery capacity of RVCs is considered to be an effective performance element by the developers, while customers do not show a strong response to the activation of it. The one design factor that produces the biggest effects when it is activated by itself is suction power. It most strongly impacts product desirability in Need Map, while it affects the attractiveness of RVCs relatively strongly and negatively in the view of the engineers. Regarding customer desirability, the second and the forth cases can be beneficial options. However, technologically, these two cases are the worst cases. On the other hand, the fifth case shows that the RVC can have a reasonable level of positive customer desirability while minimizing the negative impacts that exist from the engineers' viewpoint. The two goal concepts change in the opposite direction, which indicates a gap between what the engineers and the customers perceive to result in a better product.

In the context of the results previously, not surprisingly, such differences exist: the engineers are aware of engineering trade-offs and concerned with technical aspects of the product that the customers do not consider, which is reflected by the higher map density and the existence of feedback loops. What is surprising, though, is that the dynamic 
models based on the worldviews of the engineers and the customers lead to different conclusions. The engineers who follow the recommendations of their mental models are likely to choose solutions that are detrimental to product success. Tech Map can help marketing understand how engineering assesses product interdependencies and trade-offs, while Need Map can help developers anticipate the impacts of design decisions on product outcomes. This preliminary study thus provides some evidence that the relatively poor market penetration of RVC may, in fact, be caused by product designs that do not fulfill customer needs because engineers lack an understanding of those needs. It furthermore demonstrates the existence of cognitive distance. In addition, and in response to research question 1 (How can the cognition of market-oriented stakeholders and product development engineers be modeled?), it confirmed that FCM modeling is a means to analyze the perceptions of developers and customers not only simply as a static comparison of mental models, but also through simulations. 


\section{STUDY 2: DATA COLLECTION DURING A CDRM WORKSHOP}

Study 2 was an experimental study to evaluate the effectiveness of CDRM to answer the remaining research questions (RQ2 to RQ5) and gain practical insights into the method ${ }^{13}$. Effectiveness is defined as the ability of CDRM to help PD teams to develop a shared understanding of causal relationships between the modeled product design factors and customer preference (i.e., team mental model) that adequately represents the customers' perspective (i.e., reduced cognitive distance), so that product concepts can be generated that maximizing customer preference and can thus lead to product success.

\subsection{Experiment Overview}

Voluntary participants were engaged in an experimental product development activity. The participants' cognition of the causal relationships between PDFs and customer preference was measured through surveys at different points in the experiment (within-subject design): The first assessment occurred after the participants were introduced to the development task (through product video clips, user personas, and a product requirements document). This captured their baseline understanding before the use of CDRM. The second measurement occurred after the participants have documented their individual mental models as a cognitive map. This is the first step of CDRM method, and might already improve understanding because it causes participants to think through

\footnotetext{
${ }^{13} 2$ ) How can the cognitive models of market-oriented stakeholders and of PD engineers be integrated to represent the product concept holistically? (3) How can alternative product concepts (i.e. combinations of design characteristics) be represented in the model as alternative input scenarios? (4) How can the outcomes of alternative input scenarios be used to determine the best product concept alternative? (5) Does CDRM result in an improved and shared understanding of product design factors among product development team members?
} 
their own mental model. However, it does not permit participants to learn through interaction with other participants or model simulations. The final assessment, therefore, occurred after the participants had collaboratively used a system model of the development project, which was based on the aggregation of their individual models. My research was based on two assertions:

- In comparison to the baseline, participants improve their understanding of causal relationships between PDFs of the target product, and between PDFs and customer preference when they express their mental models as a cognitive map. This improvement demonstrates the effectiveness of the first step of CDRM.

- In comparison to the baseline and the first CDRM step, participants further improve their understanding of causal relationships between PDFs of the target product, and between PDFs and customer preference when they jointly engage with a system model of the product. This demonstrates the effectiveness of the second step of CDRM.

The comparison was based on the structural and content metrics, as well as the dynamic behavior of the models suggested by Gray et al. [220] and Yoon and Jetter [270]. Testing these assertions entailed collecting information on mental models from individual group members through cognitive mapping, aggregating the cognitive maps mathematically to represent a group mental model, and discussion and improvement of the group mental model in a workshop. In addition, three survey questionnaires were 
administered to the participants at various stages of the research to investigate their dynamic system knowledge and their perception of the process.

\subsection{Overview Study Setting and Participants of the Experiment}

The experiment was carried out with eight junior- or graduate-level engineering students from mechanical and electrical engineering, and computer science. This group size of ten is a compromise between the goal to obtain a robust data set and practicability. For focus groups, recommended group sizes are typically ten to twelve but for groups with higher levels of interaction, who need to jointly problem solve, smaller group sizes between five and eight members are recommended and ten participants are considered the upper limit [271]. .

\subsection{Experiment Process}

Figure 7.1 depicts the conceptual model of the experiment. This occurred through three experiment surveys and a day-long workshop. The first survey (Experiment Survey I) was administered online before the workshop after the participants had reviewed information on the target product (introductory movie clips, the product design requirements document, and user personas). The other two surveys were administered during the workshop, but taken individually: Experiment Survey II after participants had finished drawing their individual maps, and Experiment Survey III after the completion of a group map. The core of the surveys always remains the same and asks about the participant's perceptions about the relationships between PDFs and customer preference (see Appendix D). The second and the third surveys additionally include questions about how the participants experience the process, how they assess their own learning, and to 
what extent they agree with the group model to test the assertions developed above (see Appendix D).

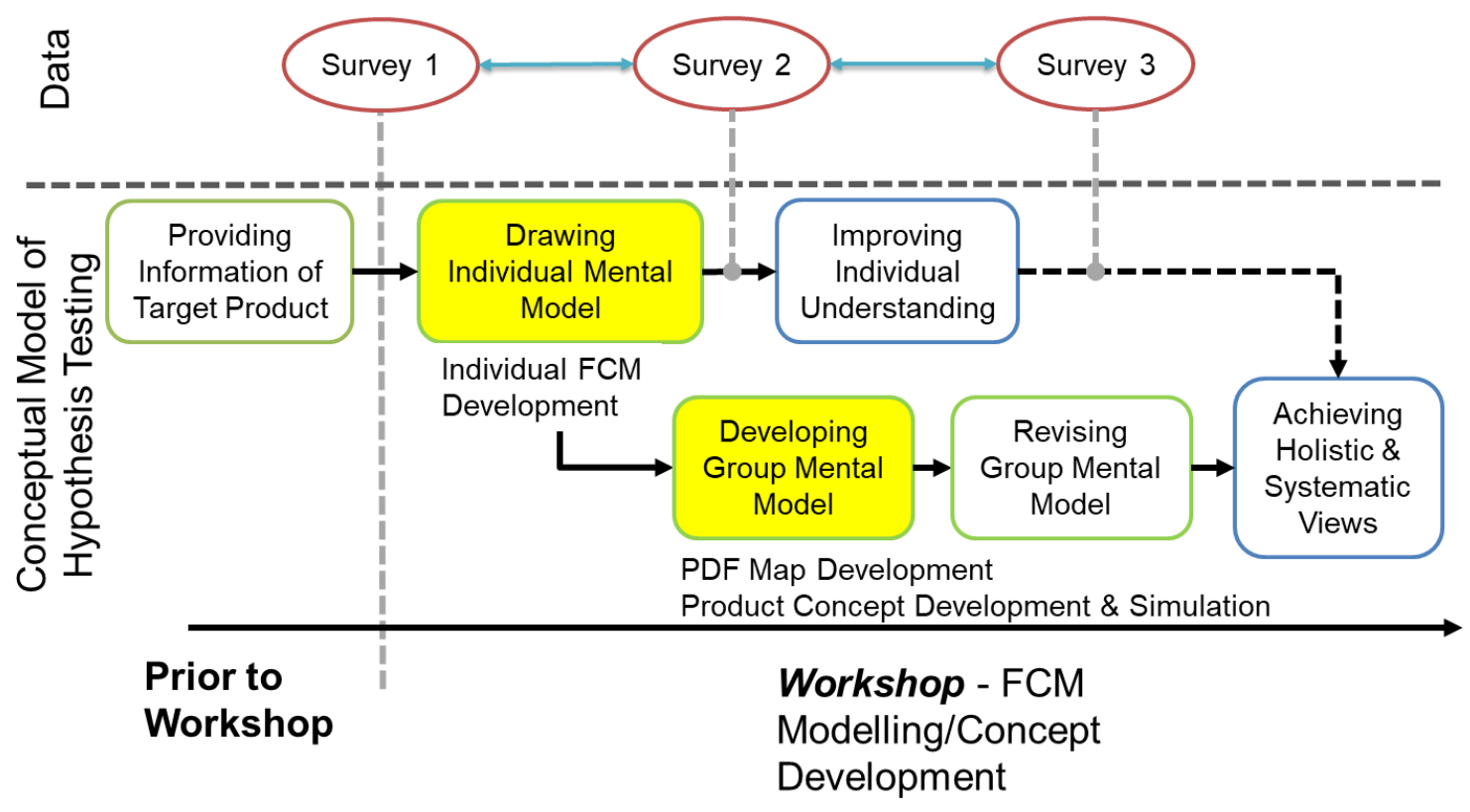

Figure 7.1 Conceptual Model of the Experiment

In parallel with the surveys, all drawings of individual and group mental models were collected and used to analyze structural and content similarities/dissimilarities between each map. The individual maps (or FCMs) were mathematically aggregated into a group FCM, providing the starting point for a focus group discussion of all participants. As part of the discussion, participants were asked to develop a group FCM by revising and modifying the mathematically aggregated map. After the workshop, the finalized group FCM and the original FCM (resulting from mathematical aggregation) were compared with each other in order to observe the change in the group mental model (i.e., the group's knowledge). 


\subsubsection{Product Information Collection}

After selecting the target product for the experiment, a home floor cleaning robot, relevant market- and technology-related information was collected in order to elicit fundamental product requirements of the target product. This information includes market research data and technology trend reports as well as interviews with subject matter experts. Specifically, a master thesis written by Eidmohammadi [272] provides customer statements and interpreted needs through studying a conceptual solution for automated vacuuming and mopping of house floors. In addition, the study of Eidmohammadi, other research papers studying customer needs on home robotic cleaning systems during the last five years are referred for product information collection [273]-[275]. Based on this collected information, a product design requirement document and user personas were developed (See Appendix B \& C). To ensure that these documents closely resemble the information used in industry, they were reviewed by an industry practitioner who is also teaching product development classes.

\subsubsection{Basic PDF Elicitation}

Basic PDFs are start concepts for the FCM modeling workshop, which were elicited from the product information described in the previous section by the researcher. Each elicited PDF is listed with a short description, as shown in Table 7.1. The start PDF list was distributed to the participants of the following workshop in a part of the handout and reviewed carefully by them during the workshop.

\section{Table 7.1. Original PDF list elicited from product information}

\begin{tabular}{clll} 
No. Category & PDF & Abb. & Description \\
\hline \hline 1 Benefit & Cleaning & CPF & The degree of performance how well a robot can clean \\
& Performance & & a specific location being set
\end{tabular}




\begin{tabular}{|c|c|c|c|}
\hline 2 Benefit & $\begin{array}{l}\text { Intelligent } \\
\text { Power } \\
\text { Management }\end{array}$ & IPM & $\begin{array}{l}\text { A combination of hardware and software that optimizes } \\
\text { the distribution and use of electrical power in a robot }\end{array}$ \\
\hline 3 Benefit & $\begin{array}{l}\text { Ease of } \\
\text { Maintenance }\end{array}$ & MTN & $\begin{array}{l}\text { The ease with which a robot can be maintained in order } \\
\text { to repair fault or worn-out components }\end{array}$ \\
\hline 4 Benefit & $\begin{array}{l}\text { Operational } \\
\text { Excellence }\end{array}$ & OPE & $\begin{array}{l}\text { The execution of cleaning more consistently and } \\
\text { reliably with using the robot than other devices }\end{array}$ \\
\hline 5 Benefit & Safety & SFT & $\begin{array}{l}\text { The state of not being dangerous or harmful in using a } \\
\text { robot }\end{array}$ \\
\hline $\begin{array}{l}6 \text { Customer } \\
\text { Preference }\end{array}$ & $\begin{array}{l}\text { Customer } \\
\text { Preference }\end{array}$ & CSP & The subjective tastes of individual consumers \\
\hline 7 Feature & $\begin{array}{l}\text { Battery } \\
\text { Capacity }\end{array}$ & $\mathrm{BCP}$ & $\begin{array}{l}\text { The maximum amount of electrical charge stored by a } \\
\text { battery unit to allow the robot to perform its task until } \\
\text { discharged }\end{array}$ \\
\hline 8 Feature & $\begin{array}{l}\text { Battery } \\
\text { Charging } \\
\text { Time }\end{array}$ & $\mathrm{BCT}$ & $\begin{array}{l}\text { A time period required to charge up the rechargeable } \\
\text { battery unit with electrical energy sufficiently or fully }\end{array}$ \\
\hline 9 Feature & $\begin{array}{l}\text { Dustbin } \\
\text { Capacity }\end{array}$ & DCP & $\begin{array}{l}\text { The maximum amount of dust stored by the dustbin the } \\
\text { robot equipped }\end{array}$ \\
\hline 10 Feature & $\begin{array}{l}\text { Energy Level } \\
\text { Indicator }\end{array}$ & ELI & $\begin{array}{l}\text { A visible indicator showing the remaining energy } \\
\text { capacity of battery }\end{array}$ \\
\hline 11 Feature & $\begin{array}{l}\text { Lower Profile } \\
\text { Design }\end{array}$ & LPD & $\begin{array}{l}\text { A thin profile design small enough to fit under the } \\
\text { couch, bed, or any type of furniture in your house }\end{array}$ \\
\hline 12 Feature & Net Weight & NWT & $\begin{array}{l}\text { Actual or estimated weight of a robot without } \\
\text { packaging }\end{array}$ \\
\hline 13 Feature & Profile Height & PHT & $\begin{array}{l}\text { The vertical distance from floor to the highest part of a } \\
\text { robot }\end{array}$ \\
\hline 14 Feature & $\begin{array}{l}\text { Suction } \\
\text { Power }\end{array}$ & SPW & $\begin{array}{l}\text { A mechanical power in the form of suction with air } \\
\text { flow, enabling to collect dust or derbies into the dustbin }\end{array}$ \\
\hline 15 Feature & $\begin{array}{l}\text { Water Tank } \\
\text { Capacity }\end{array}$ & WTC & $\begin{array}{l}\text { The maximum amount of clean water by the water tank } \\
\text { the robot equipped for cleaning purpose }\end{array}$ \\
\hline 16 Function & $\begin{array}{l}\text { Advanced } \\
\text { Navigation }\end{array}$ & ANV & $\begin{array}{l}\text { An advanced instrument that determines the position of } \\
\text { UniCleanBot and the route to a particular place }\end{array}$ \\
\hline 17 Function & $\begin{array}{l}\text { Face } \\
\text { Recognition }\end{array}$ & FRE & $\begin{array}{l}\text { The ability to automatically recognize human faces } \\
\text { based on dynamic facial images is important in security } \\
\text { and surveillance }\end{array}$ \\
\hline 18 Function & $\begin{array}{l}\text { Step-climbing } \\
\text { Mechanism }\end{array}$ & SCM & $\begin{array}{l}\text { A mechanism for enabling the robot to climb stairs and } \\
\text { move from floor to floor }\end{array}$ \\
\hline 19 Function & $\begin{array}{l}\text { Self-emptying } \\
\text { Dustbin }\end{array}$ & SED & $\begin{array}{l}\text { The ability to empty its dustbin automatically at the } \\
\text { docking station }\end{array}$ \\
\hline 20 Function & $\begin{array}{l}\text { Speech } \\
\text { Recognition }\end{array}$ & SPR & $\begin{array}{l}\text { The ability of a machine or program to identify words } \\
\text { and phrases in spoken language and convert them to a } \\
\text { UniCleanBot-readable format }\end{array}$ \\
\hline 21 Function & $\begin{array}{l}\text { Waterproof } \\
\text { Design }\end{array}$ & WPD & $\begin{array}{l}\text { Design to obtain sealing ability to protect electrical } \\
\text { parts or subsystems against water }\end{array}$ \\
\hline $\begin{array}{l}22 \text { Tech. } \\
\text { Alternative }\end{array}$ & $\begin{array}{l}\text { Expandable } \\
\text { Corner Brush }\end{array}$ & $\mathrm{ECB}$ & $\begin{array}{l}\text { A brush enabling to clean dust in corners where the } \\
\text { robot can hardly reach because of its shape or size }\end{array}$ \\
\hline $\begin{array}{l}23 \text { Tech. } \\
\text { Alternative }\end{array}$ & HEPA Filter & HEP & $\begin{array}{l}\text { High-efficiency Particulate Air Filter for individuals } \\
\text { who are allergic or asthmatic }\end{array}$ \\
\hline
\end{tabular}




\subsubsection{Need Map Development}

I developed Need Map based on the product design requirement document, user personas, and original PDF list in the previous sections. The weight on each causal relationship was determined with consideration of the context in the materials. This Need Map is presented in Chapter 9.

\subsubsection{Participant Recruitment}

The participants were recruited at Portland State University. From the experiment plan, all participants were required to have at least senior-level knowledge of engineering design or new product development, which were screened for in an online survey.

\section{Recruiting Strategy}

Participants were recruited through personal contacts and via promotional posters (see Appendix E) placed around the building of Maseeh School of Engineering and Computer Science, including the electronics prototyping lab (EPL), the Intel computer lab, and the elevators. Permission was obtained from student services. In addition, I reached out to lab managers and instructors who have frequent interactions with senior and graduate students and asked them to help the recruitment effort. With the instructor's permission, short project introductions were provided at product development- or engineering design-related classes at Portland State University. The presentations and promotional materials contained a short introduction of this experiment, project scope, eligibility of participants, incentives for participation, and contact information, including the time and date of the FCM modeling workshop. In addition, an introductory session was administrated in the Lobby of the Engineering Building. During the information sessions, a table was installed with a sign, flyers, and snacks in order to recruit passersby, 
and be available to answer questions. Some participants were invited via personal contacts and asked to suggest other participants in their network, thus implementing snowball or referral sampling.

\section{Participant Screening}

To ensure qualified participants and fulfill requirements for informed consent, volunteers were pre-screened through an online survey that each volunteer accesses through the web-link provided on all recruiting materials (see Appendix F). The screening survey includes a simple questionnaire asking for educational experiences. Just after the screening question, the survey showed an informed consent form and prompted participants to sign it if they choose to participate in the study. The consent form was approved by IRB before the survey. Participants indicated their understanding of the procedure, benefits, risks, and confidentiality of this experiment.

For participants who have been screened in and have signed the consent form, the survey gathered additional information about prior educational experience (specific courses taught by MCECS or equivalent), and work experience (see Appendix F or questions). I have implemented the survey in Qualtrics ${ }^{14}$, which permits branched survey designs: depending on their answers, participants saw different parts of the survey. Nonqualified volunteers were told that they do not qualify and received an appreciation for their interest. Qualified volunteers were invited to participate in the next steps of the experiment (i.e. following surveys and a workshop) and informed about the next steps.

\footnotetext{
${ }^{14}$ In this study, Qualtrics will be used because Portland State University is under contract with the service provider and provides this service to the researchers as a survey tool.
} 


\section{Participant Reimbursement}

While intrinsic motivators for participating, such as helping to advance research or the experience of engaging with development projects as a team, is emphasized, small extrinsic motivations such as gift cards, refreshment, and lunches were provided. The gift cards were awarded at the end of the workshop.

\subsubsection{FCM Modeling/Concept Development Workshop}

As a core part of the experiment, the FCM Modelling/Concept Development workshop was carried out on February $23^{\text {rd }}$. Followings are the details of the workshop:

\section{Participants}

The pre-screening survey was sent five days before the workshop. Consequently, twelve volunteers took the pre-screening survey, and ten of them passed it. One volunteer did not complete the survey, and another was screened because he/she does not have an engineering degree but an economics background. After a discussion with the screened volunteer, he/she was allowed to join the experiment because he/she has experience in using a vacuum cleaning robot before and been studied a technology-related topic in economics. Therefore, eleven volunteers were invited to the experiment in total. However, on the workshop day, three volunteers missed and, eight participants attended the workshop. Table 7.2 shows the brief information of each participant for this experiment. A participant ID was specified with the frequency of each participant's experience related to product development. (Note that the number in a participant ID was assigned randomly.) 


\section{Table 7.2. Participant Information of the Experiment}

\begin{tabular}{clll} 
No. & Participant ID & Academic Major & $\begin{array}{l}\text { Experience of Product } \\
\text { Development-related Projects }\end{array}$ \\
\hline 1 & E1 & Mechanical Engineering & between 2 and 4 projects \\
2 & E2 & Instrumentation Engineering & between 2 and 4 projects \\
3 & E3 & Industrial Design & over 4 projects \\
4 & E4 & Aerospace Engineering & over 4 projects \\
5 & E5 & Industrial Engineering & between 2 and 4 projects \\
6 & E6 & $\begin{array}{l}\text { Technology Management } \\
\text { Information Technology }\end{array}$ & no experience \\
7 & E7 & Management & no experience \\
8 & E8 & Economics & no experience \\
\hline
\end{tabular}

\section{Logistics}

The room for the workshop reserved has large enough to accommodate a focus group of eleven and two facilitators. The room includes one large table, projectors and whiteboard. All participants were asked to bring their own laptops. Refreshment, coffee $\&$ tea and lunch are ordered by two days before the workshop. Lastly, the gift cards for the appreciation of participation were prepared before the workshop.

\section{Pretest}

The surveys of the experiment were reviewed and tested with fellow graduate students who experienced online surveys or FCM workshops. The experiment design was reviewed carefully. In particular, the design of the workshop was monitored and revised through three "dry run" workshop with fellow students.

\section{Pre-workshop survey}

After the screening finished, an invitation email was sent to the selected participants who have agreed to participate in the workshop and have provided informed consent. It 
contains a reminder about the workshop date, time and location, questions about participant constraints (e.g. dietary restrictions), a reminder to bring their own laptop, and a Qualtrics link for the first survey (Experiment Survey I) $)^{15}$.

\section{Workshop}

The workshop was scheduled on the weekend (February $23^{\text {rd }}, 2019$ ), and lasted for about six hours (including breaks). The researcher of this dissertation served as the main facilitator of the workshop. An ETM Ph.D. student with experience in FCM workshops was recruited to support the researcher as another facilitator. Table 7.3 shows the agenda of the workshop. This table includes the product of each activity, role assignment of researchers, and materials required for each activity.

During the icebreaking, participants were encouraged to introduce themselves to other participantss. Then, a brief introduction to the workshop was presented with the research overview. Subsequently, fuzzy cognitive mapping (FCM), as the core technique of CDRM, was explained with a simple example with which enable participants to understand the process of FCM. The example was presented with MentalModeler (mentalmodeler.org), the FCM software used to facilitate the workshop. Participants also did a small exercise to practice cognitive mapping after the presentation.

\footnotetext{
15 To investigate his/her perceived understanding of the causalities between each PDF of the target product
} 
Table 7.3 Agenda of the workshop

\begin{tabular}{|c|c|c|c|c|c|}
\hline \multirow{2}{*}{ Time } & \multirow{2}{*}{ Activity Item } & \multirow{2}{*}{ Product of Activity } & \multicolumn{2}{|c|}{ Role of Researchers } & \multirow{2}{*}{ Materials } \\
\hline & & & Researcher1 & Researcher2 & \\
\hline 9:00 AM & Icebreaking & & \multirow{3}{*}{\multicolumn{2}{|c|}{ Presentation }} & Coffee \& Tea, Bagel \\
\hline \multirow[t]{2}{*}{$9: 15$ AM } & Welcome, Introduction and & & & & Handout \\
\hline & Research Overview & & & & Laptop \& Projector \\
\hline \multirow[t]{2}{*}{ 9:30 AM } & Introduction Fuzzy Cognitive & Clear understanding of & & & \\
\hline & $\begin{array}{l}\text { Mapping and Preliminary } \\
\text { Study }\end{array}$ & $\begin{array}{l}\text { FCM, as the core technique } \\
\text { of CDRM }\end{array}$ & & & \\
\hline 10:00 AM & Review PDFs & & & & \\
\hline \multirow[t]{2}{*}{ 10:30 AM } & Develop Individual Maps & Individual Maps & & Participants & Start PDF Index Cards, \\
\hline & $\begin{array}{l}\text { - (Each Participant) Develop } \\
\text { own individual map }\end{array}$ & & \multicolumn{2}{|c|}{ Collect Individual Maps } & $\begin{array}{l}\text { Blank Index Cards } \\
\text { Papers \& Pens, } \\
\text { Copy of Survey } 2 \\
\text { Individual Laptops }\end{array}$ \\
\hline 11:30 AM & Tea / Coffee Break & Experiment Survey II & \multirow{2}{*}{\multicolumn{2}{|c|}{$\begin{array}{l}\text { Collect the Survey } \\
\text { Support Participants } \\
\text { Model Coding }\end{array}$}} & Coffee \& Tea, Refreshment \\
\hline 11:45 AM & $\begin{array}{l}\text { Develop PDF Map } \\
\cdot \text { Develop a PDF Map as a } \\
\text { group map }\end{array}$ & Group Map (PDF Map) & & & $\begin{array}{l}\text { Video Recorder } \\
\text { Laptop \& projector }\end{array}$ \\
\hline $12: 30 \mathrm{AM}$ & Lunch Break & & \multirow{6}{*}{$\begin{array}{l}\text { Support } \\
\text { Participants }\end{array}$} & \multirow{5}{*}{$\begin{array}{l}\text { Verifying } \\
\text { Coded Model }\end{array}$} & Sandwich \& Beverage \\
\hline \multirow{2}{*}{ 13:00 AM } & Develop Scenarios & 3 to 5 Tentative Scenarios & & & \\
\hline & - Develop 3 to 5 scenarios & & & & \\
\hline 14:00 PM & FCM Simulation & & & & Laptop \& Projector \\
\hline 14:30 PM & Review Results of Workshop & $\begin{array}{l}\text { Experiment Survey III } \\
\text { Feedback of the Simulation } \\
\text { Results and Procedure of } \\
\text { Workshop }\end{array}$ & & & Copy of Survey 3 \\
\hline 15:00 PM & $\begin{array}{l}\text { Closing Comments and } \\
\text { Adjourn }\end{array}$ & & & & Gift \& Certificates \\
\hline
\end{tabular}


Next, the participants were introduced to the basic PDF list elicited before the workshop. After review of the list, the participants were asked if they had questions about the meaning of the proposed PDFs and were instructed on how to add and describe additional PDFs, should they see the need. In the following, the participants worked independently at tables, using their own laptops, to represent their individual mental models, aided by the facilitators who explained each step and transition between steps and answer questions. The steps for obtaining individual Maps were:

- Step 1. Selection of necessary PDFs: Each participant selected necessary PDFs for the target product from the basic PDF list ${ }^{16}$ distributed with the handout of the workshop (See Appendix G). Additionally, an mmp ${ }^{17}$ file, including all basic PDFs without any relationships was distributed to the participants through a shared folder in a web drive (google drive), which created for the experiment by the researcher. Participants downloaded and loaded the file in MentalModeler. Each PDF in the file includes the corresponding description as described in the basic PDF list, as shown in Table 7.1.

- Step 2. Setting cause-effect relationships: Each participant was required to connect two PDFs with a directional arrow with considering the causal relationship between them. For example, when PDF A affects PDF B, a directional arrow should be connected from PDF A to PDF B.

- Step 3. Weighting causal relationships: Each relationship (directional arrow) was

\footnotetext{
${ }^{16}$ Basic PDFs, as start concepts for the FCM modeling workshop, are elicited from market and technical materials relating to the target project. Each elicited PDF is written on a start PDF index card with a short description. Please refer to Figure 4.6 in the dissertation proposal.

${ }^{17}$ A file format of MentalModeler,
} 
assigned a weight that the participants chose with the help of a slider bar in the MentalModeler software. The weights were associated with a quantitative scale in the interval of -1 (strong negative) to +1 (strong positive).

- Step 4. Reviewing and tuning the mental model: Participants encouraged to review and fine-tune their individual FCMs before handing them in. When they were satisfied with their model, they saved and submitted it electronically as a MentalModeler file to the shared folder in the aforementioned web drive. Additionally, the participants were asked to save and submit their MentalModeler files as a format (comma separated values file (csv file)) that is readable with a spreadsheet program or programing languages (e.g. R and Python). Lastly, each individual FCM was screen-captured and saved an image file (png format) on the shared folder for the verification.

- Step 5. Submission of Experiment Survey II: After submitting the individual FCM, each participant was asked to take the Experiment Survey II through the Qualtrics link included in a mail sent by the researcher during the previous step.

During the lunch break after the individual FCM development activity, the researcher checked the collected individual FCMs and other files from the participants on the shared folder. Then, the researcher integrated all individual maps into a single group map as an aggregated map during the following coffee/tea break. The program for map aggregation, developed before the workshop with Python, produced a mathematically aggregated adjacency matrix. 
After the lunch break, the participants were asked to collaborate in reviewing and revising the group FCM, as a Tech Map. As a draft, the mathematically aggregated FCM was presented as a starting point of discussion in building Tech Map. With the draft map, participants reviewed all PDFs, relationships, and weights in the map. The participants checked if there were any PDFs having different titles but similar definitions and the related relationships that need to be reorganized. In addition, some weights were readjusted a during a discussion with participants until reaching an agreement. Tech Map with which all participants agreed eventually was a part of PDF Map for the target product in this experiment. Another part was Need Map developed in 7.3.3. In this activity, the researcher moderated the discussion but refrained from speaking his own opinion. The researchers finalized Tech Map and checked whether all components of the map had been correctly coded.

Next, participants discussed multiple possible combinations of fundamental PDFs as product concepts. Here, a fundamental PDF means a basic and necessary design component enabling to implement required technology alternatives, features or functions in the product design requirement document of the target product. Therefore, participants suggested multiple fundamental PDFs of which product concepts as scenarios for the following FCM simulation would be comprised.

Lastly, all participants reviewed and discussed the products (e.g., the individual and the group mental models and the product concepts) that they developed during the workshop. Moreover, they took the third survey (Experiment Survey III) asking their agreements on the group mental model, effect of the group mental model developing 
activity in addition to the perceived causalities between each PDF of the target product. After collecting the last survey, researchers awarded a gift card to each participant as a token of his/her participation in the workshop.

\subsubsection{Observations on Workshop Dynamics}

The workshop was carried out on the agenda set out in Table 7.3, but there were changes in sequence due to unforeseen circumstances. In introducing the fuzzy cognitive mapping technique to the participant, the group spent more time than scheduled 30 minutes resulted from that some of them struggled to understand causal relationships between two concepts. Moreover, a technical issue occurred in saving a map as an mmp file format during demonstrated the FCM software, MentalModeler. Fortunately, the participants were able to use the alternative saving functions with which a map is saved as a picture format (png) and its adjacency matrix is saved as a comma-separated values file (csv). The issue led to that, after the workshop, the researcher rebuilt all individual FCMs developed during the workshop referring to the picture and the csv files saved by the participants. Consequently, the delay caused a schedule change between the group map development and the lunch break.

In developing individual maps, some of the participants drew their maps considering indirect and eventual relationships rather than direct and causal ones. This indicates that they have struggled with the format of the fuzzy cognitive mapping technique. In addition, the two facilitators could not support all eight participants in developing individual maps within the scheduled time. For instance, one facilitator needed to help only one participant who had difficulty in using the FCM software while the other took care of the other seven participants. As a result, some participants might 
have drawn their individual maps without asking for support from the facilitators. Therefore, each individual FCM needed to be reviewed carefully, and, if necessary, some of them were modified after the workshop. The modification work is explained in the next chapter.

\subsubsection{Map Aggregation and Simulation}

With each individual FCM, Tech Map was developed by the mathematical aggregation tool. The aggregation tool is a Python code programmed with the algorithm described in Subsection 4.1.2. PDF Map was also developed using the Python code by aggregating Tech and Need Maps, as described in Subsection 4.2.3.

In order to analyze the behavior characteristics of each individual FCM, Tech, Need, and PDF Map, FCM simulation carried out with activating a feature/function PDF. After analyzing the characteristics of each FCM, another FCM simulations were also conducted to test alternative input scenarios as product concepts. For simulation, I utilized the Rbased FCM simulation package developed by Dikopoulou and Papageorgiou [276].

\subsubsection{Analysis \& Interpretation}

With the calculated the CPVs, the best scenario was selected, having the highest value of customer preference. These results were reviewed with PD practitioners who have experience with the product under study. Their assessment pertains to decision quality and the ability of CDRM to supplement or replace ADA: for example, if the FCM model of the PDF Map selects a product concept that they consider inferior to the other options, this would indicate that it does not adequately reflect the real-world development challenge. In addition, I reviewed the adequacy of the FCM model by analyzing the 
content, structure, and dynamic behavior of the PDF map [270]. I also assessed the impact of CDRM on individual and group mental models and thus on cognitive distance. The strategies for evaluation and analysis are further explained in the subsequent section. 


\section{STUDY 2: METHODS FOR ANALYSIS}

To answer research question 5 (Does CDRM result in an improved and shared understanding of product design factors among product development team members?) changes to the cognitive distance between participants were investigated. Specifically, three types of analyses were chosen:

- Comparison of knowledge about customer needs before the workshop (after the project was first introduced to the participants) and after the workshop: This was done by comparing questionnaire answers to track learning/cognitive distance reduction on the individual level, specifically with regard to customer/marketing knowledge. The overall approach is explained in Section 8.3 .

- Comparison of the mental model representation provided by participants at the beginning of the workshop and the team mental model resulting from the workshop: This was done by comparing map contents and structures and by tracking the overlap between team and individual mental models (i.e. the aspects of individual models that are shared with the team model).

- Comparison of the mental model representation provided by a subset of participants at the very end of the project and the team mental model, resulting from the workshop: This tracks to what extent individual embed team knowledge into their individual mental models.

The last two comparisons are based on map comparison, explained in Section 8.3. Before explaining the comparison schemes above, the next section 


\subsection{Map Refinement}

After collecting the individual FCMs from the participants, I reviewed As mentioned in Subsection 7.3.6, due to the time and the support limitation during the workshop, the individual FCMs needed to be reviewed, and, if necessary, some of them should be modified for better representation of each participant's mental model. Therefore, alternatively, I triggered a discussion about how the relationships represent the causality among the PDFs better in each FCM after sharing two individual FCMs selected randomly at the beginning of the group FCM development activity. As a result, the discussion concluded the following two items to refine each FCM:

- Focusing on direct cause-effect relationships between two PDFs

- Excluding required or eventual relationships (e.g. "a larger dustbin capacity is required if increasing suction power.”)

After the discussion, I asked the participants to have an individual meeting after the workshop to confirm the refined individual FCMs and Tech Map, then the participants agreed. Every individual FCM was reviewed carefully, then refined with the two criteria after the workshop. Every refined individual FCM was presented to each participant and asked his/her confirmation just after the refinement work was completed. As a result, all individual FCMs and Tech Map were approved by the participants at the meetings.

\subsection{Consensus Measure}

Degree of consensus can be used to track learning and understand the cognitive distance between individuals or between the same individual at different points in time. To this end, survey data about causal links between product design factors were analyzed. 
According to Tastle and Wierman, one way to define consensus is to think of it as the collective opinion of a group or a stance reached by individuals in a group [277]. Until reaching consensus, the opinion, position or understanding of each individual may change and may further converge through group decision making or a compromise process. Once consensus is reached, the group is ready to take action around an agreed-upon approach, even though there may still be some degree of variation of opinions among individuals in the group. In this study, I am using degree of consensus as a measure of a shared understanding of the causal relationships between PDFs. If CDRM is effective at reducing the cognitive distance between engineers and market-oriented stakeholders, the degree of consensus between group members should increase.

Tastle and Wierman suggested a mathematical consensus measure that can determine the degree of variation of individual positions in a group decision making based on the Likert scale [277], based on the following ground rules [278, p. 98]:

1. For a given (even) number of individuals participating in a discussion on some question of interest, if an equal number of individuals, $n / 2$, separate themselves into two disjoint teams, each centered on the strongly disagree and strongly agree categories, the team is considered to have no agreement/consensus.

2. If all the participants classify themselves in the same category of the Likert scale, regardless of the category, then the agreement/consensus of the team is considered to be complete at $100 \%$. 
3. If the mix of participants is such that $n / 2+1$ participants assign themselves to any one category, the degree of agreement/consensus must be greater than 0, for the balance in the team is no longer equal.

4. As the number of categories to which each participant classifies himself/herself diminishes, the agreement/consensus must increase, eventually approaching 1 on the unit interval. Thus, when all participants place themselves in a single category, consensus has been maximized and it considered to be perfect, and that is given a value 1 .

5. The dispersion of the categorical values must be captured by the consensus to provide an indication of the variance of the data.

With this ground rule, the consensus measure is formulated as:

$$
\operatorname{Cns}(\mathrm{X})=1+\sum_{i=1}^{n} p_{i} \log _{2}\left(1-\frac{\left|X_{i}-\mu_{x}\right|}{d_{x}}\right)
$$

Where $\mathrm{X}$ represents the list of categories, and $\boldsymbol{X}_{\boldsymbol{i}}$ is an element of $\mathrm{X}$ such as Strongly Agree (SA), Agree (A), Neutral (N), Disagree (D) and Strongly Disagree (SD). Each element has own ordinal value, e.g. $\mathrm{SD}=1, \mathrm{D}=2, \mathrm{~N}=3, \mathrm{~A}=4$ and $\mathrm{SA}=5 . \boldsymbol{p}_{\boldsymbol{i}}$ is the probability of $\boldsymbol{X}_{\boldsymbol{i}}$. Lastly, $\boldsymbol{d}_{\boldsymbol{x}}$ is the width of $\mathrm{X}\left(d_{x}=X_{\max }-X_{\min }\right)$.

Using this calculation, Table 8.1 provided as an example in the study of Tastle and Wierman [278] shows sample cases of 10 stakeholders' positions in a five-category Likert scale for a simulation of the consensus measure, and the degree of consensus corresponding to each case. 
In the CDRM process, this consensus measure, as mentioned above, allows determining a common understanding of causal relationships among PDFs between engineers and market-oriented stakeholders at a specific point of time. This measure was also applied to measure the consensus level of the participants based on the three survey responses in this research. 
Table 8.1. A simulation of the consensus measure

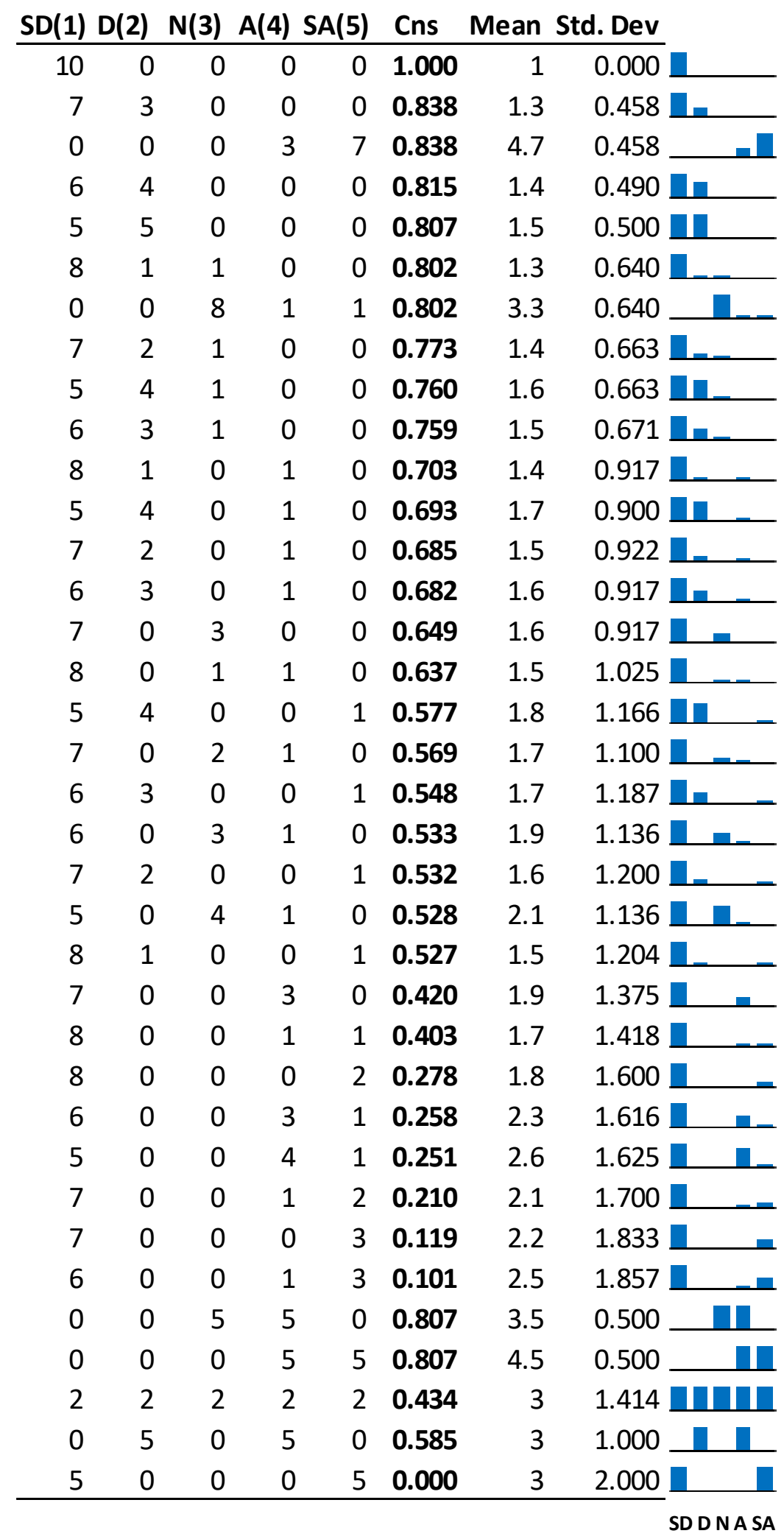




\subsection{Map Comparison and Distance Ratio}

Multiple FCMs developed for a specific topic can be compared by various metrics analyzing their structures, contents, and dynamic behaviors. These metrics for each analysis were dealt with in my previous study ${ }^{18}$ (For a more detailed discussion, please refer to the original publication). In this study, the metrics are briefly summarized to explain how the individual FCMs are compared for the experiment.

Firstly, in order to analyze the similarities or differences between the individual FCMs, their structural characteristics are compared with each other. Table 8.2 shows the main metrics, mathematical expressions, and their interpretations for structural analysis. With these metrics, it is possible to determine how densely or sparsely concepts are connected, how complicatedly the participants perceived a specific problem, and which concepts have stronger impacts on the whole system. For instance, comparing the densities of maps enables to identify which cognitive systems are more sensitive to changes in variables such as initial inputs, weights of relationships, and squashing functions.

\footnotetext{
18 This study was presented and published at Portland International Conference on Management of Engineering and Technology in 2016. (Byung Sung Yoon and A. J. Jetter, "Comparative analysis for Fuzzy Cognitive Mapping," in 2016 Portland International Conference on Management of Engineering and Technology (PICMET), 2016, pp. 1897-1908.)
} 
Table 8.2. Metrics for evaluating structural characteristics of an FCM

\section{Metrics}

\section{Definition}

Number of Concepts

(Nodes)

$N$

Total number of identical ideas having causeeffect relationships each other in a map

Out-degree

$O d_{i}=\sum_{k=1}^{N}\left|a_{i k}\right|$

The cumulative strength of connections with which a concept influences other concepts

In-degree

$$
I d_{i}=\sum_{k=1}^{N}\left|a_{k i}\right|
$$

The cumulative strength of connections through a concept is affected by other concepts

Driver

$T\left(O d_{i} \neq 0 \wedge I d_{i}=0\right)$

A concept which only influences other concepts (Forcing variables or Tails)

Receiver $\quad R\left(O d_{i}=0 \wedge I d_{i} \neq 0\right)$

A concept which is only affected by other concepts (Utility variables or Heads)

Ordinary

$O\left(O d_{i} \neq 0 \wedge I d_{i} \neq 0\right)$

A concept which is affected by and also influences other concepts

Number of

Relationships (Links)

C

Total number of connections linking each concept in a map

Density $\quad D=\frac{C}{N(N-1)} \vee D=\frac{C}{N^{2}}$

A connectivity index which shows how dense or sparse concepts are connected

Degree of Centrality $\quad C_{i}=O d_{i}+I d_{i}$

The degree how linked a concept to other concept and the cumulative strength of connections the concept has

Relationship per

Concept Ratio

(Link-Node Ratio,

Connectedness)

Receiver-Driver

Ratio (Complexity)

$C / N$

The degree of connectivity between concepts; Higher ratio indicates the connection between concepts are denser

The degree of complexity or resolution; higher ratio indicates more complex cause-effect relationships in a map

(Source: [270])

Secondly, in order to analyze the content differences between individual FCMs, it is possible to measure a distance between the adjacency matrices of two FCMs. In Linear Algebra, the Euclidean distance provides a simple similarity or difference measure between two adjacency matrices/maps. The Euclidean distance is calculated as: 


$$
\mathrm{d}(\boldsymbol{A}, \boldsymbol{B})=\left(\sum \sum\left(a_{i j}-b_{i j}\right)^{2}\right)^{1 / 2} \text { or } \mathrm{d}(\boldsymbol{A}, \boldsymbol{B})=\sum \sum\left|a_{i j}-b_{i j}\right|
$$

where A \& B are adjacency matrices of two maps having the same number of nodes, and $a_{i j}$ is the value in the ith row and the jth column of the matrices A. However, in general, each adjacency matrix may have difa ferent number of nodes with the other in measuring a similarity between two cognitive maps. In this case, the size of each adjacency matrix need to be fitted to each other to be able to calculate the distance between two matrices. According to Langfield-Smith and Wirth, the number of nodes (p) in the union of the sets of nodes in the two maps is the size of each expanded adjacency matrix, and the rows and columns which are not included in a matrix but included in the other matrix are filled with zero [279].

For example, as shown in Figure 8.1, the two maps have different numbers of nodes: map A has four concepts while map B has five concepts. The number of nodes in the union of the sets of nodes in the two matrices is six. Therefore, the adjacency matrix size of each map is expanded to six-by-six correspondingly. The row(s) and column(s) which is(are) not included in each matrix are filled with zero: the second and the fifth rows and columns in the expanded matrix of the map A; the fourth row and column in the expanded matrix of the map B. 
$\operatorname{map} \mathbf{A}$

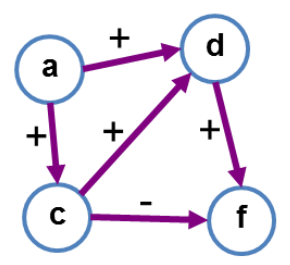

map B

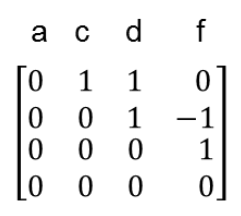

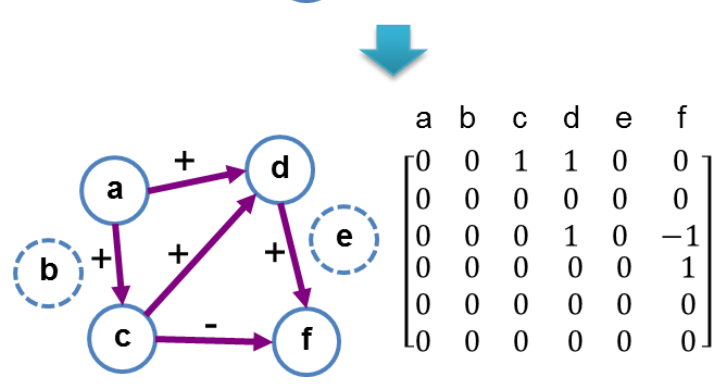
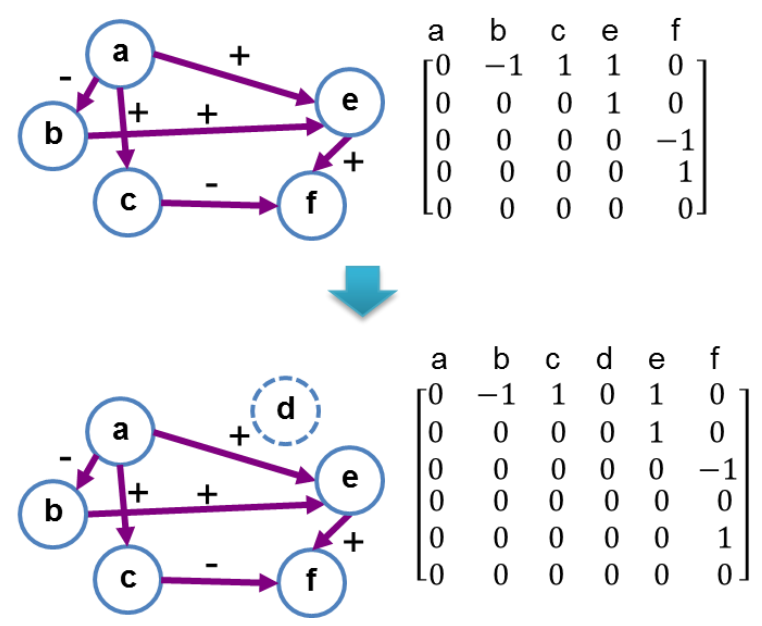

Figure 8.1 Expanded matrices for the calculation of similarity between two maps

Accordingly, the Euclidean distance is calculated as:

$$
\mathrm{d}(\boldsymbol{A}, \boldsymbol{B})=\left(\sum_{i=1}^{6} \sum_{j=1}^{6}\left(a_{i j}-b_{i j}\right)^{2}\right)^{1 / 2}=2.646
$$

However, the calculation result of the Euclidean distance does not provide a clear measure in observing the change in multiple cognitive perceptions between different junctures in the process of CDRM. In other words, because the distance measure is affected by the sizes of maps, the numbers of concepts in maps, there is a need of a relative distance, so-called a maximum distance, which normalizes the Euclidean distance to make the distance measure comparable with the other distance measure at a different time in the process of CDRM [279]. Accordingly, Langfield-Smith and Wirth suggested "Distance Ratio (DR)," which enables us to compare different pairs of maps and investigate similarities or differences of contents, concepts, and their casual relationships in maps. The formula of distance ratio is: 


$$
D R(A, B)=\frac{\sum_{i=1}^{p} \sum_{j=1}^{p}\left|a_{i j}-b_{i j}\right|}{2 p c^{2}+2 p c\left(p u_{A}+p u_{B}\right)+p u_{A}^{2}+p u_{B}^{2}-\left(2 p c+p u_{A}+p u_{B}\right)}
$$

where $\mathrm{p}$ is the number of concepts in the union of the sets of nodes in the two maps; $\mathrm{pc}$ is the number of common concepts in the two maps; and $p u_{A}$ is the number of unique nodes in the map A while $p u_{B}$ is the one in the map B. The denominator is the formula of a maximum distance which two maps can have. This maximum distance formula is formulated with consideration of that elements relating to unique concepts have a maximum difference of 1 , and those relating to common nodes have a maximum of 2 . Accordingly, the DR has a value in the rage $[0,1]$. When the DR value of a pair of maps is close to zero, the two maps are very similar while, those are very different from each other when the DR value is contiguous to one. This measure calculates the content similarity between cognitive perceptions of two different groups which, depict causal relationships among PDFs.

In addition to the DR, extracting common, partially common and unique PDFs in individual FCMs is a useful indicator to investigate the cognitive differences between individuals or groups and to indicate the degree of similarity and dissimilarity between maps on a research topic. This type of analysis enables researchers to discover cognitive commonalities or differences and draw a conclusion about decision-making behavior.

Lastly, PDF changes in FCMs (i.e., the increase or decrease of a PDF's state value) trigger changes to causally connected PDFs until the initial change impulse has made its way through the system and a new stable end point is reached. To analyze this dynamic behavior in a FCM, three metrics can be used, namely the transient response shape (a measure of the "in-between" PDF states before a new point of stability is reached), the 
ultimate states of PDFs when the termination criteria are reached, and the number of iterations elapsed from the beginning of calculation to the ultimate states. These dynamic response behaviors are dependent on the structure of the cause-effect system, initial inputs, and squashing functions applied for interaction between PDFs. 


\section{STUDY 2: RESULTS}

This chapter presents the FCMs generated in the workshop (9.1 and 9.2), the results of the surveys (9.3), the comparison of the results across these two data collection approaches (9.4), and, lastly, the feedback about the CDRM process (9.5).

\subsection{FCM Analyses}

As described in Chapter 7, each of the eight participants, in addition to taking surveys, developed and submitted his/her individual maps. Based on the collected individual FCM, a mathematically aggregated FCM was created in the workshop. The participants discussed as a group whether the elements (e.g., PDFs, relationships, and weights) of the mathematically aggregated FCM represented their world-views of the target product and revised the FCM based on the discussion. Next, multiple scenarios (i.e., product concepts) for FCM simulation were suggested. To this end, participants prioritized and combined four fundamental PDFs, intended to maximize the customer preference for the target product. The combination of PDFs was expressed as a state vector and used for FCM simulation. Simulations calculated the activation level (or state) of customer preference and all other PDFs included in the FCM.

Each of the following subchapters presents the results of these analyses steps.

\subsubsection{List of PDFs}

In the early part of the workshop, participants reviewed the list of PDFs for the floor cleaning robot. The original list shown in Table 7.1 included 23 PDFs. Through the review, the participants found that a pair of PDFs, 'Low Profile Design (LPD)' and 'Profile Height (PHT),' have similar definitions with each other. Consequently, these two 
PDFs were merged into 'Profile Height (PHT).' Moreover, the participants suggested four new PDFs which are associated with the battery and the intelligent features of UniCleanBot: 'Battery Shape (BSP),' 'Battery Size (BSZ),' 'Smart Feature (SMF),' and 'Thermo (Temperature) Recognition (THR).' All participants agreed to include these four new PDFs. Table 9.1 shows the resulting modified list of PDFs, which includes twentyeight PDFs in total.

\section{Table 9.1 Modified PDF list}

\begin{tabular}{|c|c|c|c|c|}
\hline No. & Category & PDF & Abb. & Description \\
\hline 1 & Function & $\begin{array}{l}\text { Advanced } \\
\text { Navigation }\end{array}$ & ANV & $\begin{array}{l}\text { An advanced instrument that determines the position of } \\
\text { UniCleanBot and the route to a particular place }\end{array}$ \\
\hline 2 & Feature & $\begin{array}{l}\text { Battery } \\
\text { Capacity }\end{array}$ & $\mathrm{BCP}$ & $\begin{array}{l}\text { The maximum amount of electrical charge stored by a } \\
\text { battery unit to allow the robot to perform its task until } \\
\text { discharged }\end{array}$ \\
\hline 3 & Feature & $\begin{array}{l}\text { Battery } \\
\text { Charging Time }\end{array}$ & BCT & $\begin{array}{l}\text { A time period required to charge up the rechargeable } \\
\text { battery unit with electrical energy sufficiently or fully }\end{array}$ \\
\hline 4 & Feature & Battery Shape & BSP & The spatial form of the battery \\
\hline 5 & Feature & Battery Size & BSZ & The outer space occupied by a battery unit in the robot \\
\hline 6 & Benefit & $\begin{array}{l}\text { Cleaning } \\
\text { Performance }\end{array}$ & $\mathrm{CPF}$ & $\begin{array}{l}\text { The degree of performance how well a robot can clean } \\
\text { a specific location being set }\end{array}$ \\
\hline 7 & $\begin{array}{l}\text { Customer } \\
\text { Preference }\end{array}$ & $\begin{array}{l}\text { Customer } \\
\text { Preference }\end{array}$ & CSP & The subjective tastes of individual consumers \\
\hline 8 & Feature & Coverage Area & CVA & $\begin{array}{l}\text { Area the robot can carry out a given task with a single } \\
\text { charge }\end{array}$ \\
\hline 9 & Feature & $\begin{array}{l}\text { Dustbin } \\
\text { Capacity }\end{array}$ & DCP & $\begin{array}{l}\text { The maximum amount of dust stored by the dustbin the } \\
\text { robot equipped }\end{array}$ \\
\hline 10 & $\begin{array}{l}\text { Tech. } \\
\text { Alternative }\end{array}$ & $\begin{array}{l}\text { Expandable } \\
\text { Corner Brush }\end{array}$ & ECB & $\begin{array}{l}\text { A brush enabling to clean dust in corners where the } \\
\text { robot can hardly reach because of its shape or size }\end{array}$ \\
\hline 11 & Feature & $\begin{array}{l}\text { Energy Level } \\
\text { Indicator }\end{array}$ & ELI & $\begin{array}{l}\text { A visible indicator showing the remaining energy } \\
\text { capacity of battery }\end{array}$ \\
\hline 12 & Function & $\begin{array}{l}\text { Face } \\
\text { Recognition }\end{array}$ & FRE & $\begin{array}{l}\text { The ability to automatically recognize human faces } \\
\text { based on dynamic facial images is important in security } \\
\text { and surveillance }\end{array}$ \\
\hline 13 & $\begin{array}{l}\text { Tech. } \\
\text { Alternative }\end{array}$ & HEPA Filter & HEP & $\begin{array}{l}\text { High-efficiency Particulate Air Filter for individuals } \\
\text { who are allergic or asthmatic }\end{array}$ \\
\hline
\end{tabular}




\begin{tabular}{|c|c|c|c|c|}
\hline 14 & Benefit & $\begin{array}{l}\text { Intelligent } \\
\text { Power } \\
\text { Management }\end{array}$ & IPM & $\begin{array}{l}\text { A combination of hardware and software that optimizes } \\
\text { the distribution and use of electrical power in a robot }\end{array}$ \\
\hline 15 & Benefit & $\begin{array}{l}\text { Ease of } \\
\text { Maintenance }\end{array}$ & MTN & $\begin{array}{l}\text { The ease with which a robot can be maintained in order } \\
\text { to repair fault or worn-out components }\end{array}$ \\
\hline 16 & Feature & Net Weight & NWT & Actual or estimated weight of a robot without packaging \\
\hline 17 & Benefit & $\begin{array}{l}\text { Operational } \\
\text { Excellence }\end{array}$ & OPE & $\begin{array}{l}\text { The execution of cleaning more consistently and } \\
\text { reliably with using the robot than other devices }\end{array}$ \\
\hline 18 & Feature & Profile Height & PHT & $\begin{array}{l}\text { The vertical distance from floor to the highest part of a } \\
\text { robot }\end{array}$ \\
\hline 19 & Feature & $\begin{array}{l}\text { Product } \\
\text { Volume }\end{array}$ & PRV & The amount of space occupied by the robot \\
\hline 20 & Function & $\begin{array}{l}\text { Step-climbing } \\
\text { Mechanism }\end{array}$ & $\mathrm{SCM}$ & $\begin{array}{l}\text { A mechanism for enabling the robot to climb stairs and } \\
\text { move from floor to floor }\end{array}$ \\
\hline 21 & Function & $\begin{array}{l}\text { Self-emptying } \\
\text { Dustbin }\end{array}$ & SED & $\begin{array}{l}\text { The ability to empty its dustbin automatically at the } \\
\text { docking station }\end{array}$ \\
\hline 22 & Benefit & Safety & SFT & $\begin{array}{l}\text { The state of not being dangerous or harmful in using a } \\
\text { robot }\end{array}$ \\
\hline 23 & Feature & Smart Feature & SMF & $\begin{array}{l}\text { A feature that aid in controlling, operating, or } \\
\text { monitoring the robot's tasks or functions }\end{array}$ \\
\hline 24 & Function & $\begin{array}{l}\text { Speech } \\
\text { Recognition }\end{array}$ & SPR & $\begin{array}{l}\text { The ability of a machine or program to identify words } \\
\text { and phrases in spoken language and convert them to a } \\
\text { UniCleanBot-readable format }\end{array}$ \\
\hline 25 & Feature & Suction Power & SPW & $\begin{array}{l}\text { A mechanical power in the form of suction with air } \\
\text { flow, enabling to collect dust or debris into the dustbin }\end{array}$ \\
\hline 26 & Function & $\begin{array}{l}\text { Thermo } \\
\text { Recognition }\end{array}$ & THR & The ability to sense temperature of a working space \\
\hline 27 & Function & $\begin{array}{l}\text { Waterproof } \\
\text { Design }\end{array}$ & WPD & $\begin{array}{l}\text { Design to obtain sealing ability to protect electrical } \\
\text { parts or subsystems against water }\end{array}$ \\
\hline 28 & Feature & $\begin{array}{l}\text { Water Tank } \\
\text { Capacity }\end{array}$ & WTC & $\begin{array}{l}\text { The maximum amount of clean water by the water tank } \\
\text { the robot equipped for cleaning purpose }\end{array}$ \\
\hline
\end{tabular}

\subsubsection{Individual FCMs}

Eight individual FCMs were collected from the participants during the workshop. Participants referred to the modified list of PDFs for UniCleanBot but were not required to include all PDFs in their individual maps. Each FCM and its adjacency matrix is shown in Appendix I. Every individual FCM was refined after the workshop based on the criteria described in Section 8.1, and Appendix $\mathrm{J}$ includes the refined individual FCMs and their 
adjacency matrixes. The refined individual maps were used for the analyses described below.

For a start, eight metrics measuring the overall structural characteristics of an FCM were calculated as summarized in Table 9.2.

Table 9.2 Structural characteristics evaluation for the individual FCMs

\begin{tabular}{|c|c|c|c|c|c|c|c|c|}
\hline 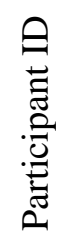 & $\frac{\sqrt[c]{1}}{0}$ & 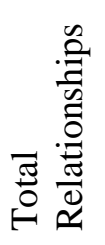 & 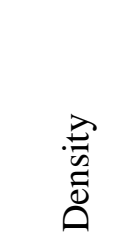 & 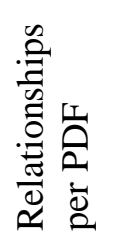 & 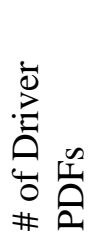 & 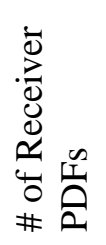 & 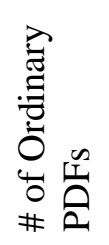 & 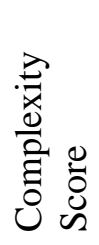 \\
\hline $\mathrm{E} 1$ & 24 & 32 & 0.058 & 1.333 & 9 & 4 & 11 & 0.444 \\
\hline E2 & 22 & 36 & 0.078 & 1.636 & 8 & 6 & 8 & 0.750 \\
\hline E3 & 26 & 39 & 0.060 & 1.500 & 15 & 5 & 6 & 0.333 \\
\hline E4 & 24 & 64 & 0.116 & 2.667 & 6 & 3 & 15 & 0.500 \\
\hline E5 & 22 & 40 & 0.087 & 1.818 & 5 & 1 & 16 & 0.200 \\
\hline E6 & 20 & 21 & 0.055 & 1.050 & 8 & 7 & 5 & 0.875 \\
\hline E7 & 21 & 19 & 0.045 & 0.905 & 6 & 10 & 5 & 1.667 \\
\hline E8 & 22 & 34 & 0.074 & 1.545 & 11 & 4 & 7 & 0.364 \\
\hline
\end{tabular}

(Note: An orange cell indicates the largest number among the eight individual FCMs while a blue cell means the smallest one.)

The total PDF numbers of the eight FCMs are distributed between 20 and 26, while the total relationship numbers are between nineteen and sixty-four. The FCM drawn by the participant E3 has the largest number of PDFs, twenty-six, while N6's FCM has the smallest, twenty. Furthermore, E4's FCM has the largest number of relationships, sixtyfour, while E7's has the smallest, nineteen. As a result, E4's FCM is the densest, while E7's is the sparsest relatively. On the other hand, with regard to the complexity revealing the ratio of receiver to driver PDFs in an FCM, E7's FCM is the most complex, while 
E5's one is the simplest ${ }^{19}$. The reason that E5's FCM has such a high complexity is that it has a larger number of receivers than drivers.

Next, the degree of centrality of each PDF was calculated. It provides a measure of the number of connections between a PDF and other PDFs. Figure 9.1 presents the degree of centrality of each PDF in all eight individual FCMs. As can be seen in the charts, there are few instances of "across-the-board" high or low centrality concepts. However, referring to the participants' information summarized in Table 7.2, the FCMs developed by the three participants who have no experience in product development related projects have less degree of centrality on average compared to the others.

${ }^{19}$ According to Özesmi and Őzesmi [229], a complex FCM with large complexity involves more utility outcomes while less controlling forcing functions. 
Degree of Centrality in E1's FCM

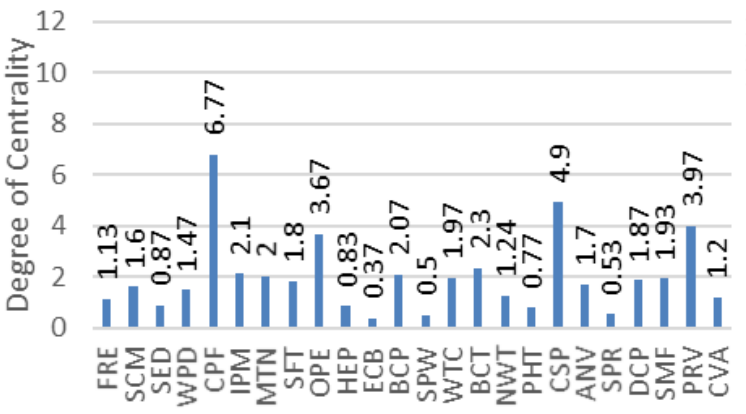

Degree of Centrality in E3's FCM

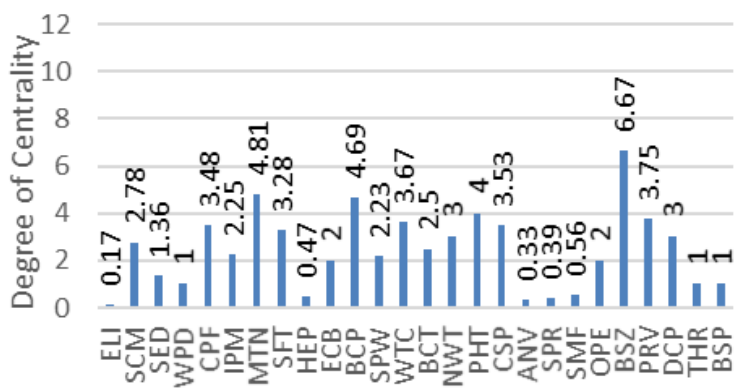

Degree of Centrality in E5's FCM

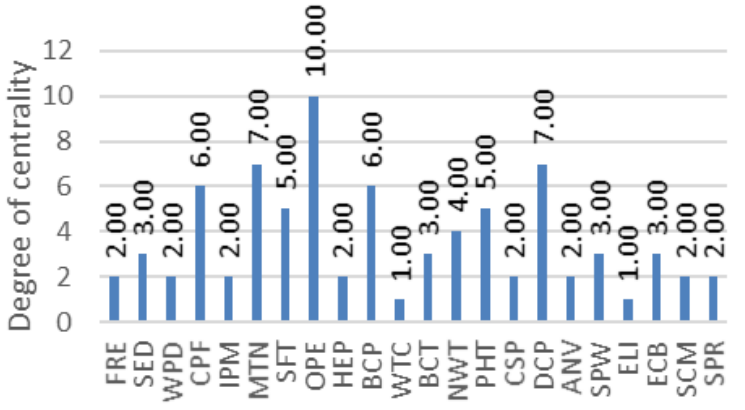

Degree of Centrality in E7's FCM

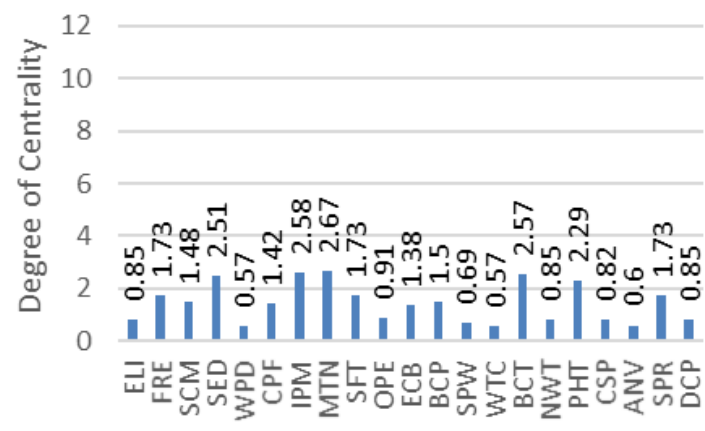

Name of PDF
Degree of Centrality in E2's FCM

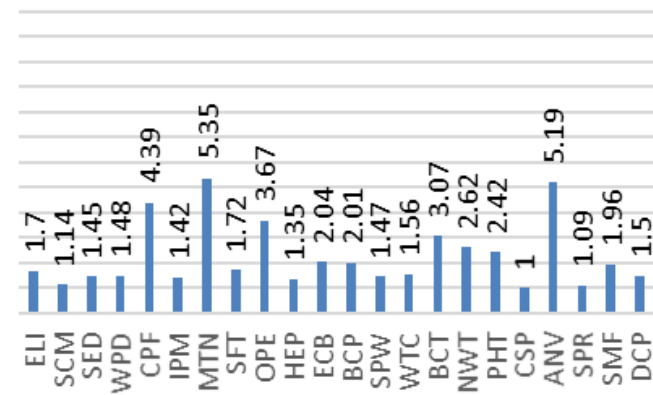

Degree of Centrality in E4's FCM

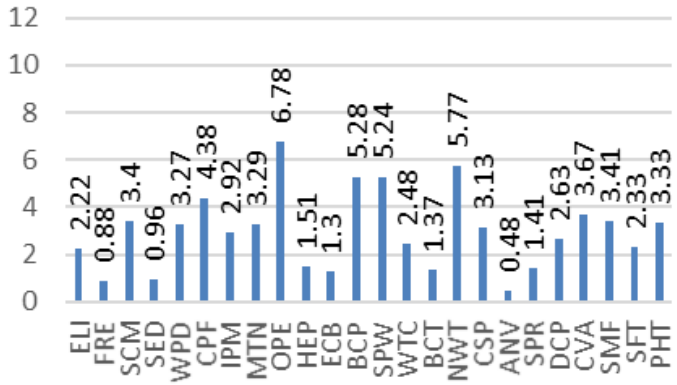

Degree of Centrality in E6's FCM

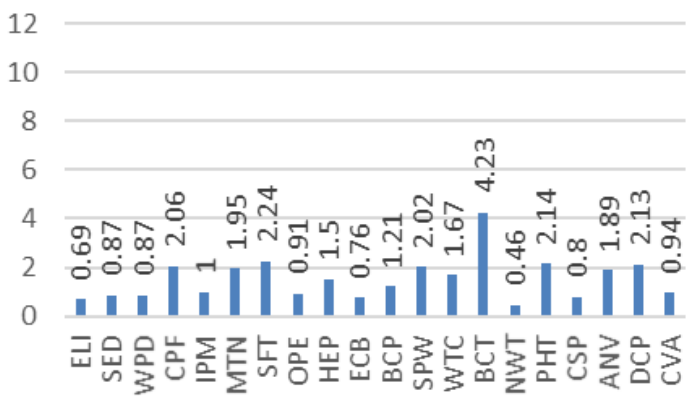

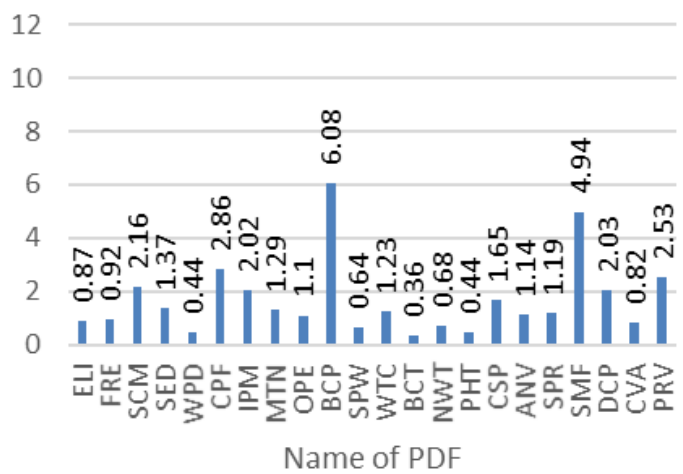

Figure 9.1 Degree of Centrality of each PDF in the eight individual FCMs 
Of particular interest are PDFs that represent product benefits of UniCleanBot, according to the provided marketing information, namely 'Cleaning Performance (CPF)', 'Operational Excellence (OPE)', Intelligent Power Management (IPM)', 'Maintenance (MTN)', and 'Safety (SFT)'). Figure 9.2 shows the degrees of centrality for these five benefits in each individual FCM.

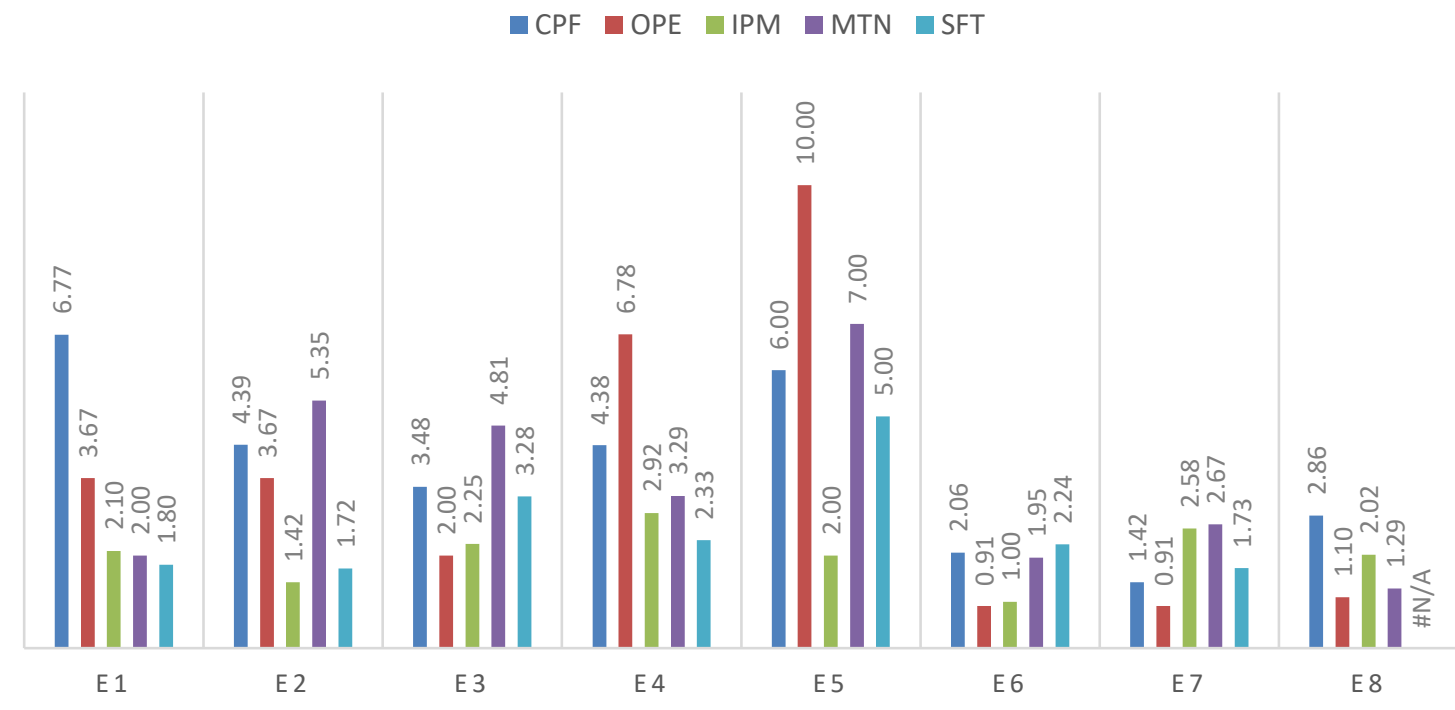

Figure 9.2 Degree of centrality of five main desired benefits in each participant's FCM

Again, no strong pattern emerges: According to the chart in Figure 9.2, participant E1 connected a larger number of causal relationships to CPF relatively than the other benefit-related PDFs. Whereas, OPE has larger relationships in E4's and E5's FCMs relatively. In E2's and E3's FCMs, MTN is the largest relationship among the five desired benefits. However, in E6's, E7's and E8's FCMs, the centrality values do not differ much, compared with other FCMs.

To compare the contents of an individual FCM with another, a distance ratio between a pair of FCMs was calculated with Equation (9). For this calculation, two FCMs 
(E4 and E7) out of the eight were selected considering the structural differences in density analyzed in Table 9.2. Table 9.3 presents the distance ration calculation result $(0.081)$ between E4's and E7's FCMs.

Table 9.3 Distance ratio between E7's and E4's FCMs

\begin{tabular}{|c|c|c|}
\hline & \multicolumn{2}{|c|}{ FCM } \\
\hline Comparison Criteria & E4 & E7 \\
\hline Number of PDFs & 24 & 21 \\
\hline Number of Relationships & 64 & 19 \\
\hline $\mathrm{C} / \mathrm{N}$ Value & 2.667 & 0.905 \\
\hline Density & 0.116 & 0.045 \\
\hline Number of Drivers & 6 & 6 \\
\hline Number of Receivers & 3 & 10 \\
\hline Number of Ordinaries & 15 & 5 \\
\hline Complexity Score & 0.5 & 1.667 \\
\hline Distance Ratio & \multicolumn{2}{|c|}{0.081} \\
\hline Number of PDFs in Distant Matrix & \multicolumn{2}{|c|}{24} \\
\hline Number of Common PDFs to Both Matrices & \multicolumn{2}{|c|}{21} \\
\hline Number of Unique PDFs in E7 & \multicolumn{2}{|c|}{0} \\
\hline Number of Unique PDFs in E4 & \multicolumn{2}{|c|}{3} \\
\hline
\end{tabular}

According to the table, E4's FCM includes all PDFs in E7's while it has three unique PDFs that E7 does not: 'Coverage Area (CVA),' 'HEPA Filter (HEP),' and 'Smart Feature (SMF),' Though the difference in numbers of relationships between the two FCMs is big, the distance ratio shows that these FCMs are similar to each other because the distance ratio is closer to zero than to one associated with their contents. However, a distance ratio can be deployed as a measure for a relative comparison of content differences among multiple FCMs. Table 9.4 summarizes the relative distance ratios between two individual FCMs. From the table, the distance ratio between E4's and E8's 
FCMs is the largest compared to the others. Whereas, E3's FCM has the closest distance ratio with E1's. In other words, E7's FCM is more similar to E3's than E4's with respect to content. Additionally, with this comparison of distance ratios among multiple pairs of FCMs, it is possible to observe the change in distance ratio between a pair of individual FCMs developed at different times. This comparison is discussed in the following analysis section.

Table 9.4 Relative comparison of distance ratios between individual FCMs

\begin{tabular}{l|lllllllll} 
& E1 & \multicolumn{1}{l}{ E2 } & E3 & E4 & E5 & E6 & E7 & \multicolumn{2}{l}{ E8 } \\
\hline E1 & & 0.055 & 0.041 & 0.072 & 0.051 & 0.046 & 0.050 & 0.054 \\
E2 & 0.055 & & 0.051 & 0.087 & 0.063 & 0.058 & 0.056 & 0.070 \\
E3 & 0.041 & 0.051 & & 0.072 & 0.051 & 0.049 & 0.050 & 0.058 \\
E4 & 0.072 & 0.087 & 0.072 & & 0.087 & 0.085 & 0.081 & 0.088 \\
E5 & 0.051 & 0.063 & 0.051 & 0.087 & & 0.063 & 0.060 & 0.073 \\
E6 & 0.046 & 0.058 & 0.049 & 0.085 & 0.063 & & 0.045 & 0.062 \\
E7 & 0.050 & 0.056 & 0.050 & 0.081 & 0.060 & 0.045 & & 0.059 \\
E8 & 0.054 & 0.070 & 0.058 & 0.088 & 0.073 & 0.062 & 0.059 &
\end{tabular}

Finally, it is important to investigate the dynamic behavior of each individual FCM to understanding its characteristics. In this section, each refined individual FCM is simulated with activating each of two PDFs (HEPA Filter (HEP) and Advanced Navigation (ANV)) categorized as a technological alternative or a function, mainly drivers, in order to understand its entire systematical dynamic behavior. In other words, the simulation result of each simulation presents the responses of the upper-level PDFs (CPF, OPE, IPM, MTN, SFT, and CSP) to the activation of the two drivers (HEP and ANV). Because E7's and E8's FCMs do not include HEP, they were excluded from the 
analysis. The six remaining FCMs were simulated with a hyperbolic tangent function $(\lambda$ $=2$ ) described in Equation (6) as the squashing function.

Each of the six plots in Figure 9.3 shows the behavior of the responses of the upperlevel PDFs to the activation of HEP. For example, the activation of HEP has no impact on the upper-level PDFs in E3's FCM depicted in Figure 9.3 (c). Whereas, the other plots show that the activation of HEP results in changing the values of some upper-level PDFs negatively or positively. In E3's FCM depicted in Figure 9.4, HEP is connected to PRV (Product Volume), but there is no outgoing arrow from PRV. Consequently, HEP has no connection with the upper-level PDFs in E3's FCM, which is presented by the simulation result. Similarly, CSP is not affected by the activation of HEP in E2's FCM, as depicted in Figure 9.3 (b). 


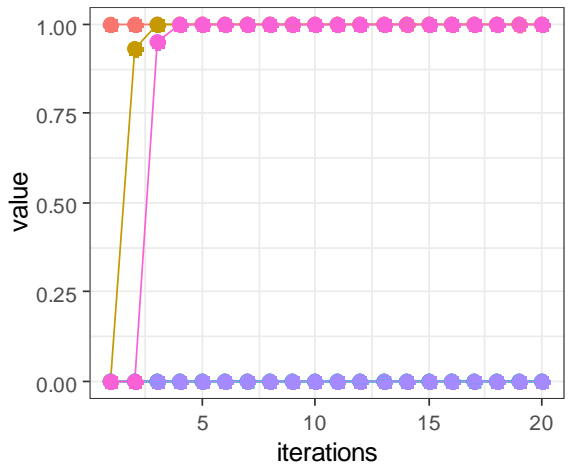

(a) E1

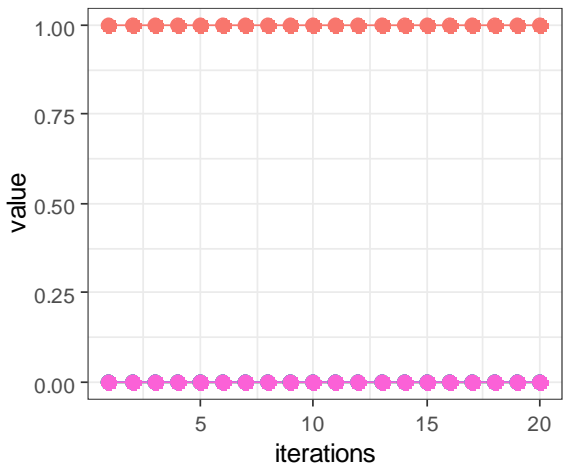

(c) E3

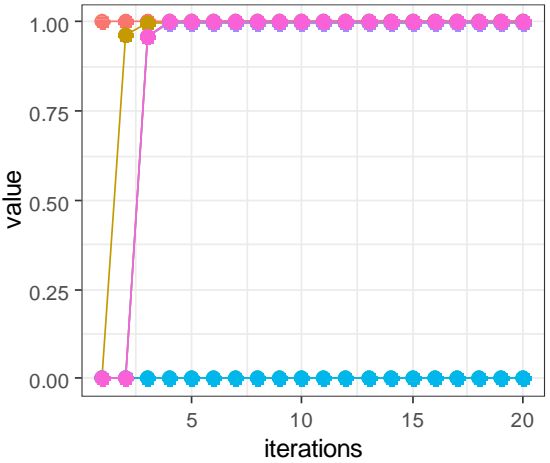

(e) E5

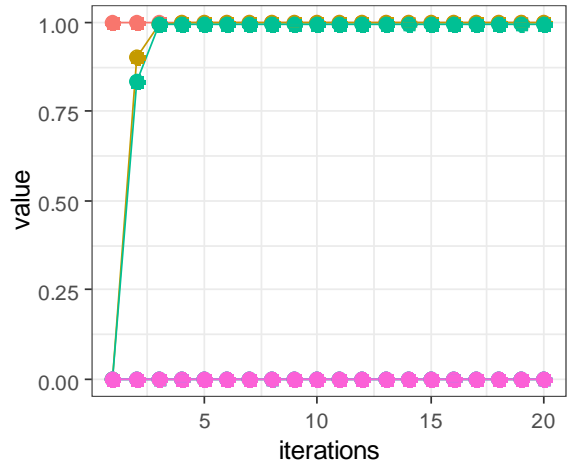

(b) E2

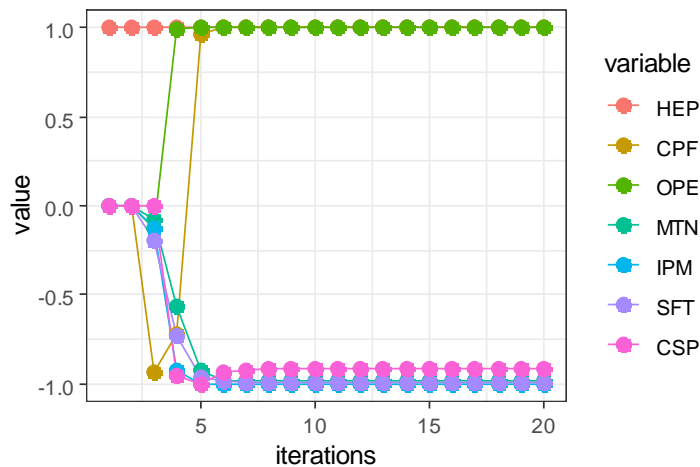

(d) E4

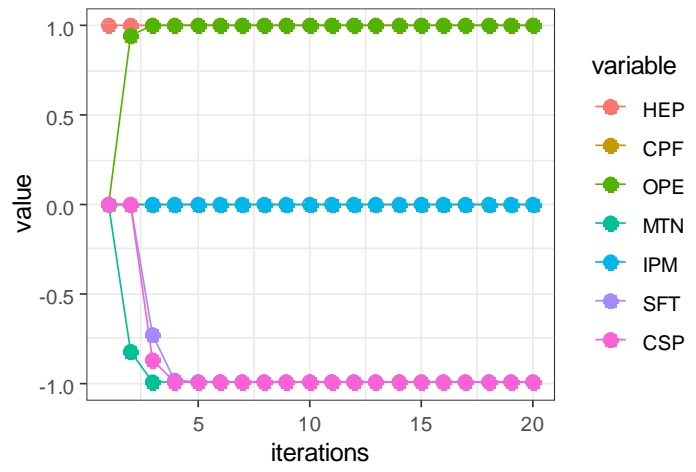

(f) E6

Figure 9.3 Simulation responses to activation HEP 


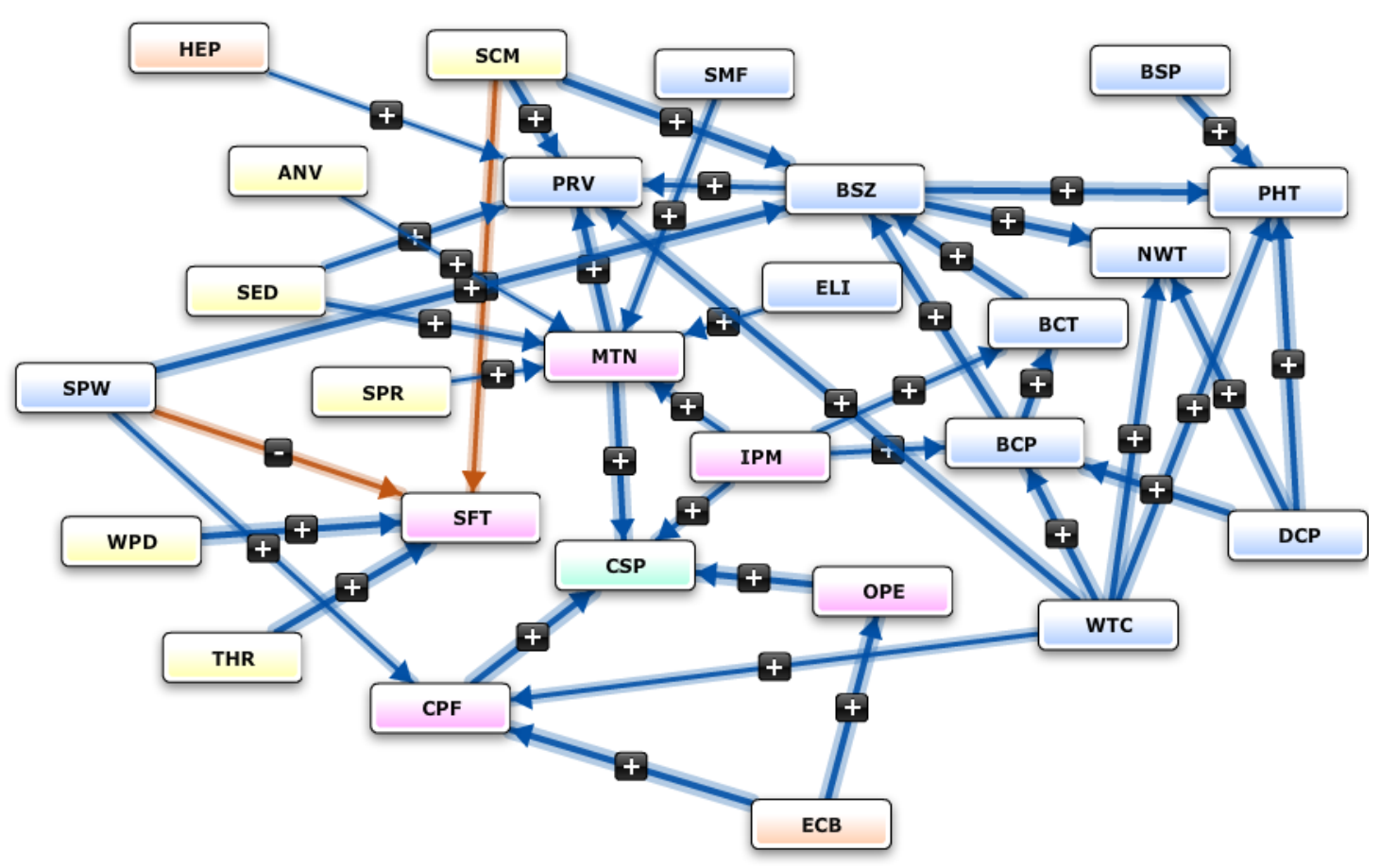

Figure 9.4 E3's FCM

Results were determined through a stop rule, namely when the system either reaches a stable state in all its concepts or meets a stop criterion. In this study, the stop rule was that a simulation is terminated when the difference between the state value of each PDF at an iteration and at the previous one is less than 0.00001. At this point, the final value for each concept and the number of iterations it took to reach stability were summarized in Table 9.5.

Table 9.5 Settled responses \& converged iteration number in each six FCM

\begin{tabular}{ccccccccc}
$\begin{array}{l}\text { Participant's } \\
\text { ID }\end{array}$ & HEP & CPF & OPE & IPM & MTN & SFT & CSP & $\begin{array}{l}\text { Converged } \\
\text { Iteration Number }\end{array}$ \\
\hline E1 & 1.000 & 0.999 & 0.000 & 0.000 & 0.000 & 0.000 & 0.999 & 6 \\
E2 & 1.000 & 0.998 & 0.000 & 0.000 & 0.997 & 0.000 & 0.000 & 6 \\
E3 & 1.000 & 0.000 & 0.000 & 0.000 & 0.000 & 0.000 & 0.000 & 1 \\
E4 & 1.000 & 1.000 & 1.000 & -0.998 & -0.984 & -0.987 & -0.912 & 16 \\
E5 & 1.000 & 0.999 & 1.000 & 0.000 & 0.000 & 0.999 & 1.000 & 6 \\
E6 & 1.000 & 0.000 & 0.999 & 0.000 & -0.996 & -0.996 & -0.998 & 8 \\
\hline
\end{tabular}


In Table 9.5, the activation of HEP affects CPF positively and very strongly in E1's, E2's, E4's, and E5's FCM while OPE is affected positively and very strongly in E4's, E5's, and E6's. IPM is affected strongly and negatively by HEP in only E4's FCM. Lastly, CSP is influenced positively by HEP in E1's and E5's FCM, negatively in E4's and E6's. Regarding the converged iteration number of simulation, E4's responses reach a stable status after 16 iterations, meaning that the simulation took a lot of iterations relatively compared with the other FCMs, resulted from the complicate structure of the FCM.

In order to observe the response behaviors, E6's and E7's FCMs, these two were simulated with the activation of ANV by the same way of the previous simulation, as presented in Figure 9.5 and Table 9.6.

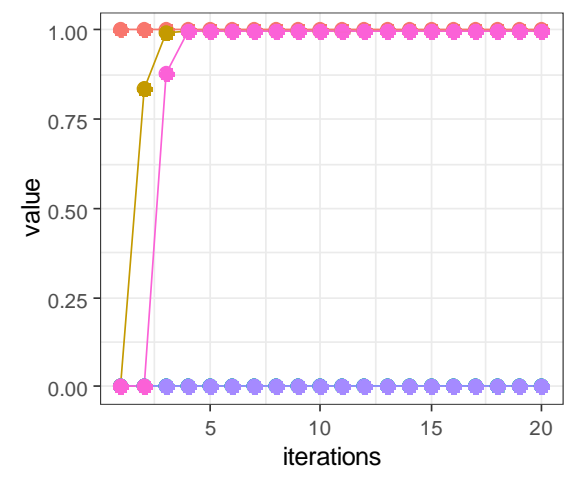

(a) E7

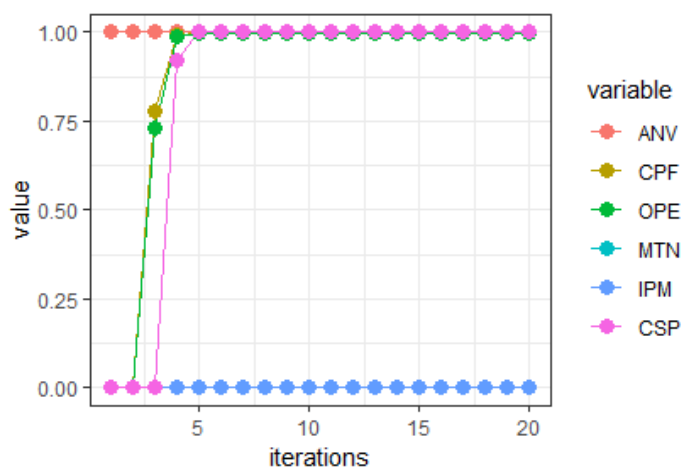

(b) E8

Figure 9.5 Simulation responses to activation ANV

Table 9.6 Settled responses \& converged iteration number in E7's and E8's FCM

\begin{tabular}{ccccccccc}
$\begin{array}{l}\text { Participant's } \\
\text { ID }\end{array}$ & ANV & CPF & OPE & IPM & MTN & SFT & CSP & $\begin{array}{l}\text { Converged } \\
\text { Iteration Number }\end{array}$ \\
\hline E7 & 1.000 & 0.997 & 0.000 & 0.000 & 0.000 & 0.000 & 0.999 & 7 \\
E8 & 1.000 & 0.996 & 0.995 & 0.000 & 0.000 & - & 0.999 & 12 \\
\hline
\end{tabular}

The activation of ANV results in the positive responses of OPE and CSP in E7's FCM, whereas affecting OPE, IPM, and CSP positively in E8's. Moreover, the responses 
of OPE and IPF start to change from the second iteration in E8's FCM, and the CSP response begins to react from the fourth one. On the other hand, the responses of CFP and CSP start to respond from the first and the second iterations, respectively in E7's FCM. The responses of E8's FCM in simulation reach a stable status after the $12^{\text {th }}$ iteration while one of E7's settles after the $7^{\text {th }}$ iteration.

To sum up, each individual FCM collected from the eight participants at the workshop has different characteristics of contents, structure, and behavior from each other. The differences between each individual FCM have been confirmed by multiple analyses, such as structural characteristics evaluation, centrality analysis, distance ratio calculation, and behavior analysis, in this section. The next section presents Tech Map constructed by aggregating the eight individual FCM, and the results of analyses for Tech Map.

\subsubsection{Group Maps - Tech and Need Maps}

Figure 9.6 depicts Tech Map drawn by aggregating the eight individual FCMs mathematically using the Python code. Because MentalModeler does not provide a tool for aggregating multiple FCMs, I developed the Python code. For visualization of the aggregated FCM, Tec, I deployed two R packages, igraph [280] and visNetwork [281]. The corresponding adjacency matrix of Tech Map is presented in Table 9.7.

I, as a market-oriented stakeholder, developed Need Map based on the product design requirements document and user personas provided to the participants of the workshop, as depicted in Figure 9.7. Table 9.8 is the corresponding adjacency matrix. 


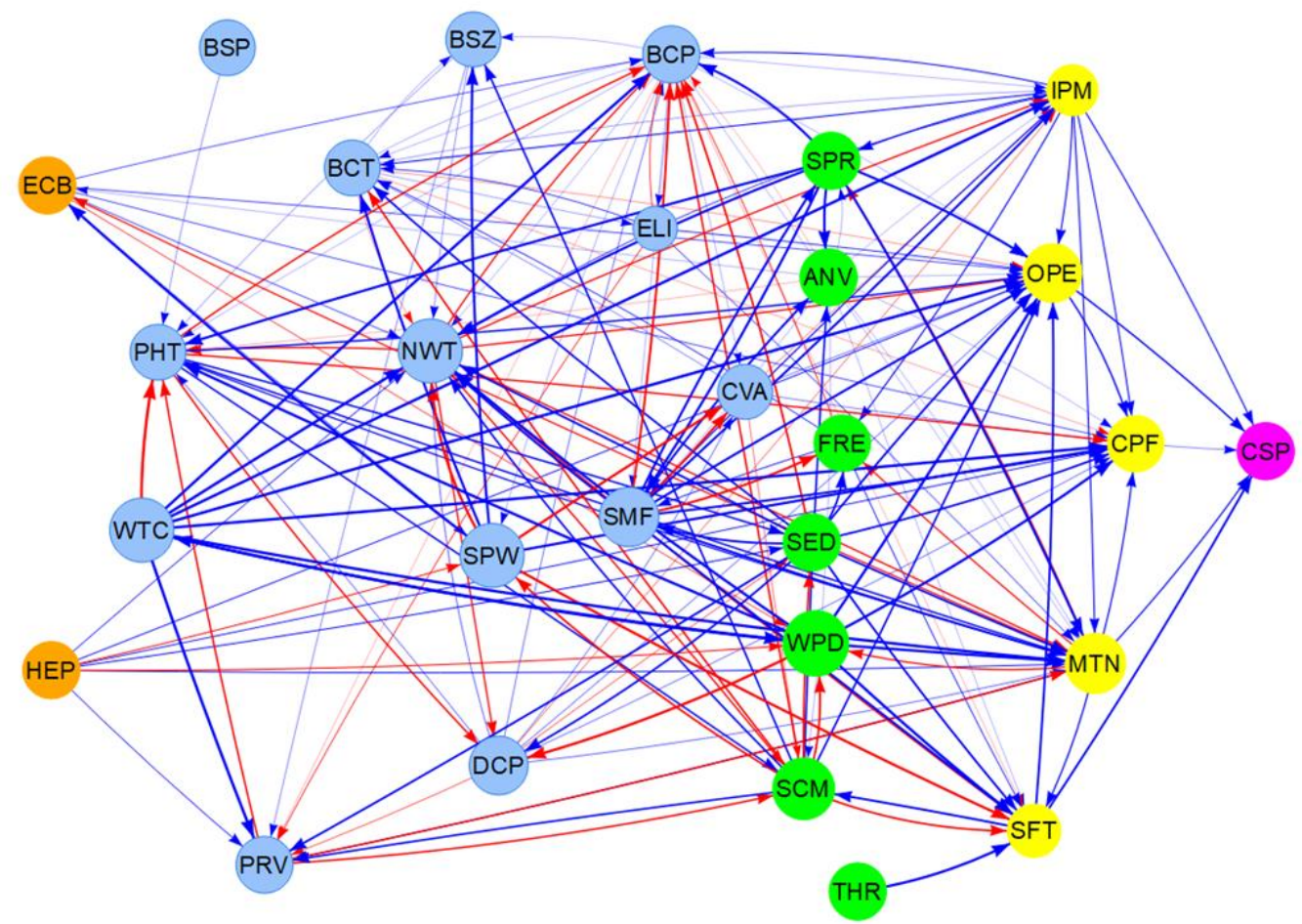

Figure 9.6 Tech Map

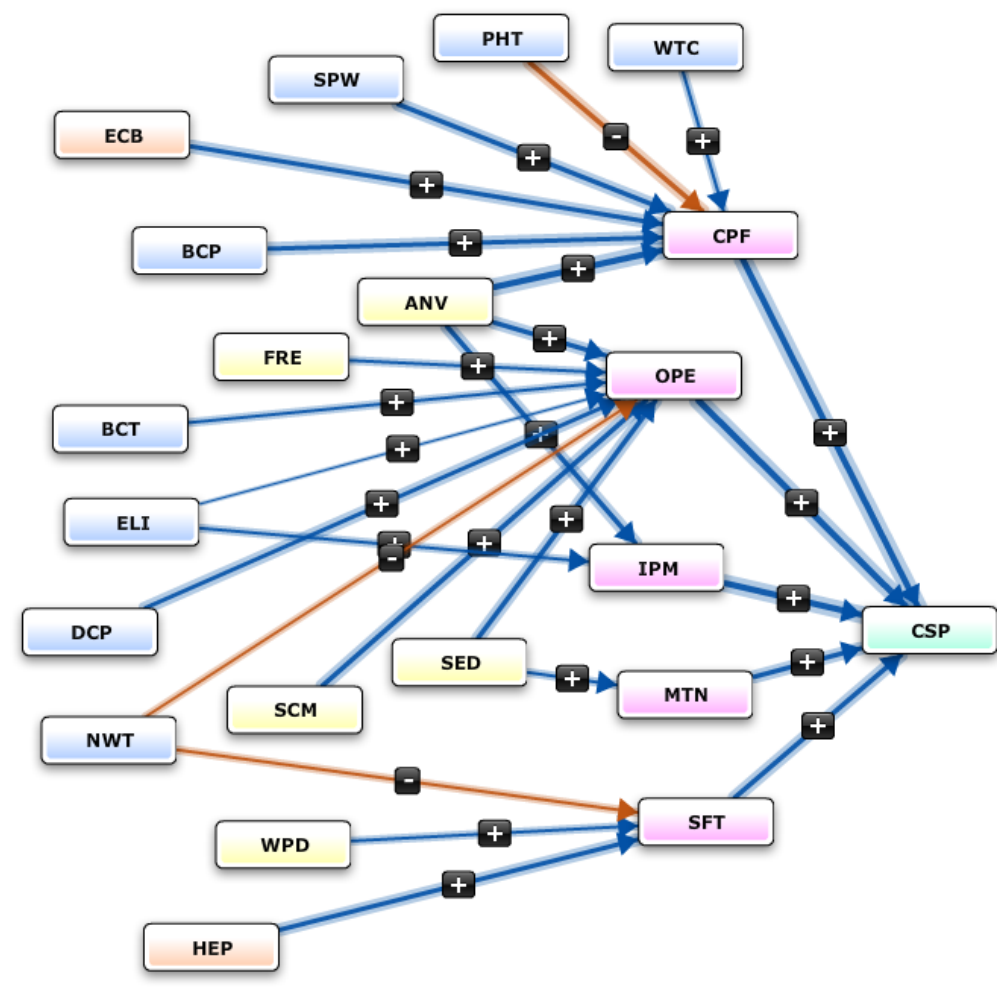

Figure 9.7 Need Map 
Table 9.7 Adjacency matrix of Tech Map

\begin{tabular}{|c|c|c|c|c|c|c|c|c|c|c|c|c|c|c|c|c|c|c|c|c|c|c|c|c|c|c|c|c|c|c|}
\hline & ANV & $\mathrm{BCP}$ & $\mathrm{BCT}$ & BSP & BSZ & & CPF & CSP & CVA & DCP & ECB & ELI & FRE & HEP & IPM & MTN & NWT & OPE & PHT & PRV & $\mathrm{SCM}$ & SED & SFT & SMF & SPR & SPW & THR & & VPD & WTC \\
\hline ANV & & -0.595 & 0 & & 0 & 0 & 0.756 & 0 & 0 & 0 & 0.5 & 0 & 0 & 0 & 0.55 & 0.41 & 0 & 0.87 & -0.099 & 0 & 0.8 & 0 & 0.68 & 0.65 & 0.44 & 0 & 0 & 0 & 0 & 0 \\
\hline $\mathrm{BCP}$ & 0 & 0 & 0.842 & & 0 & 1 & 0 & 0 & 1 & 0 & 0 & 0.77 & 0 & 0 & 0.18 & 0.305 & 1 & 0.83 & $\begin{array}{ll}3 & 1\end{array}$ & -0.62 & 0 & & -0.325 & 0.75 & 0 & 1 & 1 & 0 & 0 & 0 \\
\hline BCT & 0 & 0.795 & 0 & & 0 & 1 & -0.5 & 0 & 0 & 0 & 0 & 0.89 & 0 & 0 & 1 & 0 & -0.62 & -0.286 & 0 & 0 & 0 & 0 & 0 & 0 & 0 & 0 & 0 & 0 & 0 & 0 \\
\hline BSP & 0 & 0 & 0 & & 0 & 0 & 0 & 0 & 0 & 0 & 0 & 0 & 0 & 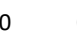 & 0 & 0 & 0 & 0 & 1 & 0 & 0 & 0 & 0 & 0 & 0 & 0 & 0 & 0 & 0 & 0 \\
\hline BSZ & 0 & 0 & 0 & & 0 & 0 & 0 & 0 & 0 & 0 & 0 & 0 & 0 & 0 & 0 & 0 & 1 & 0 & 1 & 0.75 & 0 & 0 & 0 & 0 & 0 & 0 & 0 & 0 & 0 & 0 \\
\hline CPF & 0 & 0 & 0 & & 0 & 0 & 0 & 0.823 & 0 & 0 & 0 & 0 & 0 & ( & 0 & 0 & 0 & 0 & 0 & 0 & 0 & 0 & 0 & 0 & 0 & 0 & 0 & 0 & 0 & 0 \\
\hline CSP & 0 & 0 & 0 & & 0 & 0 & 0 & 0 & 0 & 0 & ) & 0 & 0 & & 0 & 0 & 0 & 0 & 0 & 0 & 0 & 0 & 0 & 0 & 0 & 0 & 0 & 0 & 0 & 0 \\
\hline CVA & 0 & 0 & 0.57 & & 0 & 0 & 0.41 & 0 & 0 & 0 & 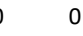 & 0 & 0 & & $\begin{array}{ll}0 & -0.74\end{array}$ & 0 & 0 & 0.7 & 0 & 0 & -0.82 & 0 & 0 & 0 & 0 & 0 & 0 & 0 & 0 & 0 \\
\hline DCP & 0 & 0.285 & 0 & & 0 & 0 & 0.713 & 0 & 1 & 0 & 0 & 0 & 0 & & $\begin{array}{ll}0 & -0.58\end{array}$ & 0.896 & 0.732 & 1 & 0.399 & -0.019 & 0 & -0.58 & 0 & 0 & 0 & 0 & 0 & 0 & 0 & 0 \\
\hline ECB & 0 & 0.69 & 0 & & 0 & 0 & 0.78 & 0 & 0 & 0 & 0 & 0 & 0 & & $\begin{array}{ll}0 & 0\end{array}$ & 0 & 0.08 & 1 & 0 & 0 & 0 & 0 & 0 & 0 & 0 & 0.605 & & 0 & -0.7 & 0 \\
\hline ELI & 0 & -0.44 & 0.69 & & 0 & 0 & 0 & 0 & 0 & 0 & 0 & 0 & 0 & & 0.52 & 0.18 & 0.37 & 0.7 & 0 & -0.43 & 0 & 0 & 0 & 0 & 0 & 0 & 0 & 0 & 0 & 0 \\
\hline FRE & 0 & -0.32 & 0.73 & & 0 & 0 & 0 & 0 & 0 & 0 & 0 & 0 & 0 & & 0 & 0 & 0 & 0 & 0 & 0 & 0 & 0 & 0.28 & 0.6 & 0 & 0 & 0 & 0 & 0 & 0 \\
\hline HEP & 0 & 0 & 0 & & 0 & 0 & 0.86 & 0 & 0 & 0 & c & 0 & 0 & & 0 & 0.005 & 0.18 & 0.91 & 0 & 0.47 & 0 & 1 & 0 & 0 & 0 & -0.89 & & 0 & -0.44 & 0 \\
\hline IPM & 0 & 0.179 & 0.453 & & 0 & 0 & 0.85 & 0.723 & 0 & 0 & 0 & 0 & 0.82 & & 0 & 0.759 & 0 & 1 & 0 & 0 & 0 & 0 & 0 & 0.75 & 0.88 & 0 & & 0 & 0 & 0 \\
\hline MTN & 0 & 0 & 0 & & 0 & 0 & 0.69 & 0.754 & 0 & 0 & -1 & 0 & -1 & & 0 & 0 & 0 & 0 & 0.94 & 1 & 0 & 0 & 0.56 & 0 & -0.925 & 0 & & 0 & -1 & 0 \\
\hline NWT & 0 & -0.84 & 0 & & 0 & 0 & 0 & 0 & 0 & -0.075 & 0 & 0 & 0 & & $\begin{array}{ll}0 & -0.19\end{array}$ & -0.11 & 0 & -0.18 & $\begin{array}{ll}3 & -0.79\end{array}$ & 0 & -0.77 & 0 & -0.41 & 0 & 0 & 0 & & 0 & 0 & 0 \\
\hline OPE & 0 & 0 & 0 & & 0 & 0 & 1 & 0.926 & 0 & 0 & 0 & 0 & 0 & & $\begin{array}{ll}0 & 0\end{array}$ & 0 & 0 & 0 & ) 0 & 0 & 0 & 0 & 0 & 0 & 0 & 0 & & 0 & 0 & 0 \\
\hline PHT & 0 & -1 & 0 & & 0 & 0 & -0.28 & 0 & 0 & -1 & 1 & 0 & 0 & & 0 & 1 & 0 & 0.065 & 0 & 0 & 0.6 & 0.75 & 0 & 0 & 0 & 0 & & 0 & 0 & 0 \\
\hline PRV & 0 & 0 & 0 & & 0 & 0 & 0 & 0 & 0 & 0 & 0 & 0 & 0 & & 0 & -1 & 0 & 0 & -0.77 & 0 & -0.83 & 0 & 0 & 0 & 0 & 0 & & 0 & 0 & 0 \\
\hline SCM & 0.48 & -0.51 & -0.37 & & 0 & 1 & 0 & 0 & 0 & 0 & 0 & 0 & 0 & & 0 & 0 & 1 & 1 & 0 & 0.305 & 0 & -0.44 & -0.66 & 0 & 0 & -0.52 & & $\begin{array}{ll}0 & -0\end{array}$ & 0.074 & 0 \\
\hline SED & 0 & -0.35 & 0.75 & & 0 & 0 & 0.87 & 0 & 0 & 0.87 & 0 & 0 & 0.91 & & 0 & 0.764 & 0.52 & 1 & 0 & 0.53 & 0 & 0 & 0.925 & 0.44 & 0 & 0 & & 0 & 0 & 0 \\
\hline SFT & 0 & 0 & 0 & & 0 & 0 & 0 & 0.695 & 0 & 0 & 0 & 0 & 0 & & 0 & 0 & 0 & 1 & 0 & 0 & 0.88 & 0 & 0 & 0 & 0 & 0 & & 0 & 0 & 0 \\
\hline SMF & 1 & -0.37 & 0 & & 0 & 0 & 0.6 & 0 & -0.3 & 0 & 0 & 0 & -0.019 & & 0.37 & 0.459 & 0.15 & 0.77 & 0 & 0 & 0 & 0 & 0.18 & 0 & 0.53 & 0 & & 0 & 0 & 0 \\
\hline SPR & 1 & 0.08 & 0 & & 0 & 0 & 0 & 0 & 0 & 0 & 0 & 0 & 0 & & 0 & 0.39 & 0.26 & 0.33 & 0.08 & 0 & ( & 0 & 0 & 0.63 & 0 & 0 & & 0 & 0 & 0 \\
\hline SPW & 0 & 0 & 0.09 & & 0 & .92 & 0.74 & 0 & -0.52 & 0 & 0.77 & 7 & 0 & & 0 & 0 & -0.89 & 0 & 0 & 0 & 0 & 0 & -0.69 & 0 & 0 & 0 & & 0 & 0 & 0 \\
\hline THR & 0 & 0 & 0 & & 0 & 0 & 0 & 0 & 0 & 0 & 0 & 0 & 0 & & 0 & 0 & 0 & 0 & 0 & 0 & 0 & 0 & 1 & 0 & 0 & 0 & & 0 & 0 & 0 \\
\hline WPD & 0 & 0 & 0 & & 0 & 0 & 0.47 & 0 & 0 & -0.3 & 0 & 0 & 0 & & 0 & 0.47 & 0.56 & 0.605 & 0.38 & 0 & 0 & 0 & 0.933 & 0 & 0 & 0 & & 0 & 0 & 0.285 \\
\hline WTC & 0 & 0.21 & 0 & & 0 & 0 & 0.89 & 0 & 0 & 0 & 0 & 0 & 0 & & 0.18 & 1 & 0.835 & & -0.229 & 0.159 & 0 & 0 & 0 & 0 & 0 & 0 & & 0 & 0.57 & 0 \\
\hline
\end{tabular}


Table 9.8 Adjacency matrix of Need Map

\begin{tabular}{|c|c|c|c|c|c|c|c|c|c|c|c|c|c|c|c|c|c|c|c|c|c|}
\hline & CSP & CPF & OPE & IPM & MTN & ANV & $\mathrm{SCM}$ & SED & HEP & $\mathrm{ECB}$ & ELI & FRE & PHT & WPD & $\mathrm{BCP}$ & $\mathrm{BCT}$ & SPW & WTC & $D C P$ & & NWT \\
\hline CSP & 0 & 0 & 0 & 0 & 0 & 0 & 0 & 0 & 0 & 0 & 0 & 0 & 0 & 0 & 0 & 0 & 0 & 0 & 0 & 0 & 0 \\
\hline CPF & 1 & 0 & 0 & 0 & 0 & 0 & 0 & 0 & 0 & 0 & 0 & 0 & 0 & 0 & 0 & 0 & 0 & 0 & 0 & 0 & c \\
\hline OPE & 1 & 0 & 0 & 0 & 0 & 0 & 0 & 0 & 0 & 0 & 0 & 0 & 0 & 0 & 0 & 0 & 0 & 0 & 0 & 0 & c \\
\hline IPM & 1 & 0 & 0 & 0 & 0 & 0 & 0 & 0 & 0 & 0 & 0 & 0 & 0 & 0 & 0 & 0 & 0 & 0 & 0 & 0 & c \\
\hline MTN & 0.79 & 0 & 0 & 0 & 0 & 0 & 0 & 0 & 0 & 0 & 0 & 0 & 0 & 0 & 0 & 0 & 0 & 0 & 0 & 0 & c \\
\hline SFT & 0.79 & 0 & 0 & 0 & 0 & 0 & 0 & 0 & 0 & 0 & 0 & 0 & 0 & 0 & 0 & 0 & 0 & 0 & 0 & 0 & c \\
\hline ANV & 0 & 0.86 & 0.79 & 0.51 & 0 & 0 & 0 & 0 & 0 & 0 & 0 & 0 & 0 & 0 & 0 & 0 & 0 & 0 & 0 & 0 & c \\
\hline SCM & 0 & 0 & 0.76 & 0 & 0 & 0 & 0 & 0 & 0 & 0 & 0 & 0 & 0 & 0 & 0 & 0 & 0 & 0 & 0 & 0 & c \\
\hline SED & 0 & 0 & 0.76 & 0 & 0.41 & 0 & 0 & 0 & 0 & 0 & 0 & 0 & 0 & 0 & 0 & 0 & 0 & 0 & 0 & 0 & c \\
\hline HEP & 0 & 0 & 0 & 0 & 0 & 0.62 & 0 & 0 & 0 & 0 & 0 & 0 & 0 & 0 & 0 & 0 & 0 & 0 & 0 & 0 & c \\
\hline$E C B$ & 0 & 0.51 & 0 & 0 & 0 & 0 & 0 & 0 & 0 & 0 & 0 & 0 & 0 & 0 & 0 & 0 & 0 & 0 & 0 & 0 & c \\
\hline ELI & 0 & 0 & 0.13 & 0.34 & 0 & 0 & 0 & 0 & 0 & 0 & 0 & 0 & 0 & 0 & 0 & 0 & 0 & 0 & 0 & 0 & c \\
\hline FRE & 0 & 0 & 0.2 & 0 & 0 & 0 & 0 & 0 & 0 & 0 & 0 & 0 & 0 & 0 & 0 & 0 & 0 & 0 & 0 & 0 & c \\
\hline PHT & 0 & -0.53 & 0 & 0 & 0 & 0 & 0 & 0 & 0 & 0 & 0 & 0 & 0 & 0 & 0 & 0 & 0 & 0 & 0 & 0 & c \\
\hline WPD & 0 & 0 & 0 & 0 & 0 & 0.41 & 0 & 0 & 0 & 0 & 0 & 0 & 0 & 0 & 0 & 0 & 0 & 0 & 0 & 0 & c \\
\hline $\mathrm{BCP}$ & 0 & 0.55 & 0 & 0 & 0 & 0 & 0 & 0 & 0 & 0 & 0 & 0 & 0 & 0 & 0 & 0 & 0 & 0 & 0 & 0 & c \\
\hline $\mathrm{BCT}$ & 0 & 0 & 0.44 & 0 & 0 & 0 & 0 & 0 & 0 & 0 & 0 & 0 & 0 & 0 & 0 & 0 & 0 & 0 & 0 & 0 & c \\
\hline SPW & 0 & 0.83 & 0 & 0 & 0 & 0 & 0 & 0 & 0 & 0 & 0 & 0 & 0 & 0 & 0 & 0 & 0 & 0 & 0 & 0 & c \\
\hline WTC & 0 & 0.48 & 0 & 0 & 0 & 0 & 0 & 0 & 0 & 0 & 0 & 0 & 0 & 0 & 0 & 0 & 0 & 0 & 0 & 0 & $c$ \\
\hline$D C P$ & 0 & 0 & 0.58 & 0 & 0 & 0 & 0 & 0 & 0 & 0 & 0 & 0 & 0 & 0 & 0 & 0 & 0 & 0 & 0 & 0 & c \\
\hline NWT & 0 & 0 & -0.46 & 0 & 0 & -0.49 & 0 & 0 & 0 & 0 & 0 & 0 & 0 & 0 & 0 & 0 & 0 & 0 & 0 & 0 & c \\
\hline
\end{tabular}


Table 9.9 summarizes the structural analysis of Tech and Need Map. Tech Map is comprised of 28 PDFs and 182 relationships. The number of the relationships is considerably large so that the $\mathrm{C} / \mathrm{N}$ value (6.5) and the density (0.241) of Tech Map are also high. It includes three drivers (BSP, THR, and HEP) and only one receiver (CSP), resulting in 0.333 of the complexity score. The number of the relationships is relatively small compared to Tech Map so that the $\mathrm{C} / \mathrm{N}$ value (1.19) and the density (0.06) of Need Map are also low. Moreover, it includes 15 drivers and only one receiver (CSP), resulting in 0.067 of the complexity score, which means that Need Map is less complicated than Tech Map.

Table 9.9 Structural analysis of Tech and Need Map

\begin{tabular}{rrr} 
& Tech Map & Need Map \\
\hline Number of PDFs & 28 & 21 \\
Number of Relationships & 182 & 25 \\
C/N Value & 6.5 & 1.19 \\
Density & 0.241 & 0.06 \\
Number of Drivers & 3 & 15 \\
Number of Receivers & 1 & 1 \\
Number of Ordinaries & 24 & 25 \\
Complexity Score & 0.333 & 0.067 \\
\hline
\end{tabular}

Figure 9.8 represents the centralities (in-degree, out-degree centrality and degree of centrality) of each PDF in Tech and Need Map. Tech Map, the upper chart, has relatively high in-degree centralities of CPF (10.41) and OPE (13.25) while it has high out-degree centralities of BCP (9.62), MTN (7.87) and SED (7.93). Moreover, BCP, MTN, and OPE have high degrees of centrality $(16.29,15.62$, and 15.17 correspondingly) in Tech Map. On the other hand, Need Map, the bottom chart, has relatively high in-degree centralities of CSP (4.58), OPE (4.12) and CPF (3.76) while it has high out-degree centrality of ANV 
(2.16). OPE, CPF, and CSP have high degrees of centrality $(5.12,4.76$, and 4.58 correspondingly) in Need Map.
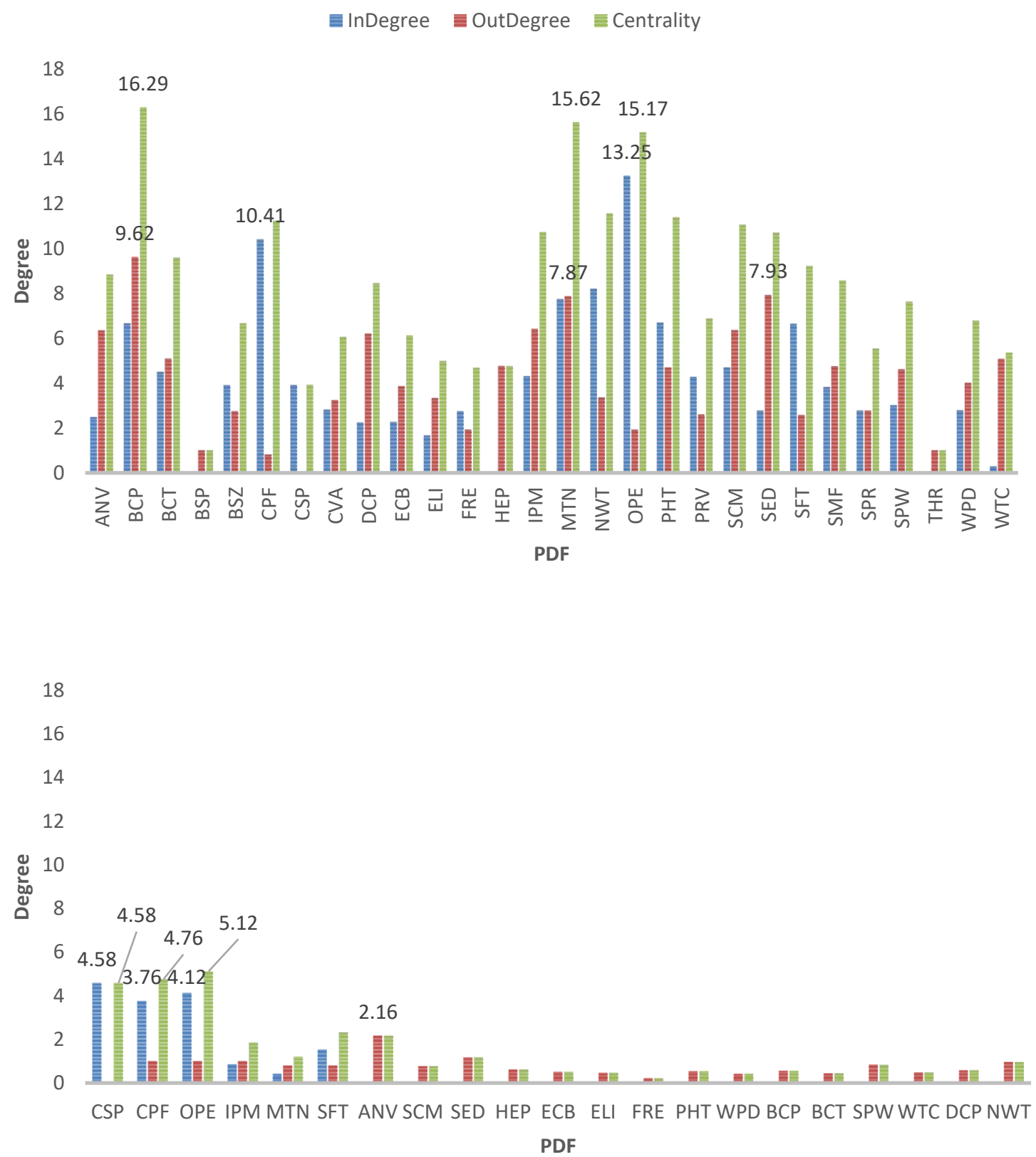

Figure 9.8 Centrality analysis of Tech (upper) and Need (bottom) Maps

The responses of the six upper-level PDFs to the activation of BCP were simulated to investigate the dynamic behavior characteristics of Tech and Need Maps. Figure 9.9 
shows the simulation result about the dynamic behavior of Tech and Need Maps, and Table 9.10 summarizes the response value of the six upper-level PDFs at the point of stability, as well as the number of iterations it takes for results to converge.
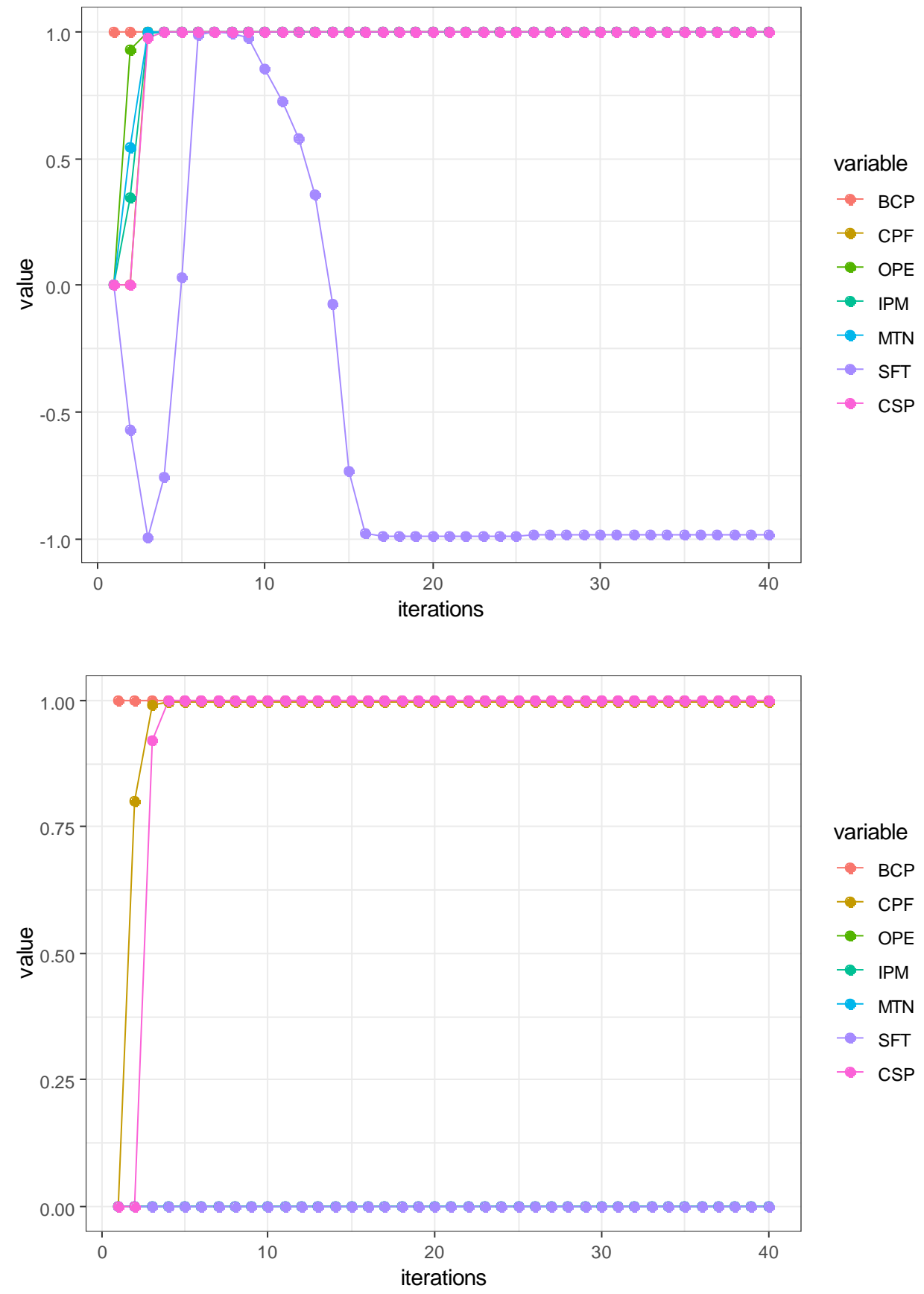

Figure 9.9 Dynamic behavior of Tech and Need Maps 
Table 9.10 Settled responses \& converged iteration number in Tech Map

\begin{tabular}{lrrrrrrrc} 
& BCP & CPF & OPE & IPM & MTN & SFT & CSP & $\begin{array}{l}\text { Converged } \\
\text { Iteration Number }\end{array}$ \\
\hline Tech Map & 1.000 & 1.000 & 1.000 & 1.000 & 1.000 & -0987 & 1.000 & 36 \\
Need Map & 1.000 & 0.996 & 0 & 0 & 0 & 0 & 0.999 & 6 \\
\hline
\end{tabular}

The activation of BCP in Tech Map resulted in the very positive value of CSP and four upper-level PDFs such as CPF, OPE, IPM, and MTN, whereas SFT has a negative value $(-0.987)$. In addition, the response of simulation converged after the $36^{\text {th }}$ iteration. Specifically, the response of SFT converged slower compared to other PDFs. In contrast, the activation of BCP in Need Map resulted in the very positive value of CSP (0.999) and CSP (0.996), whereas the other upper-level PDFs remained zero. The response of the simulation converged after the $6^{\text {th }}$ iteration, which is faster compared to the Tech Map's response.

\subsection{FCM Analyses of PDF Map and Simulation}

\subsubsection{PDF Map}

Figure 9.10 depicts PDF Map drawn by aggregating Tech and Need Maps using the Python code used for Tech Map development. Table 9.11 shows the corresponding adjacency matrix. 


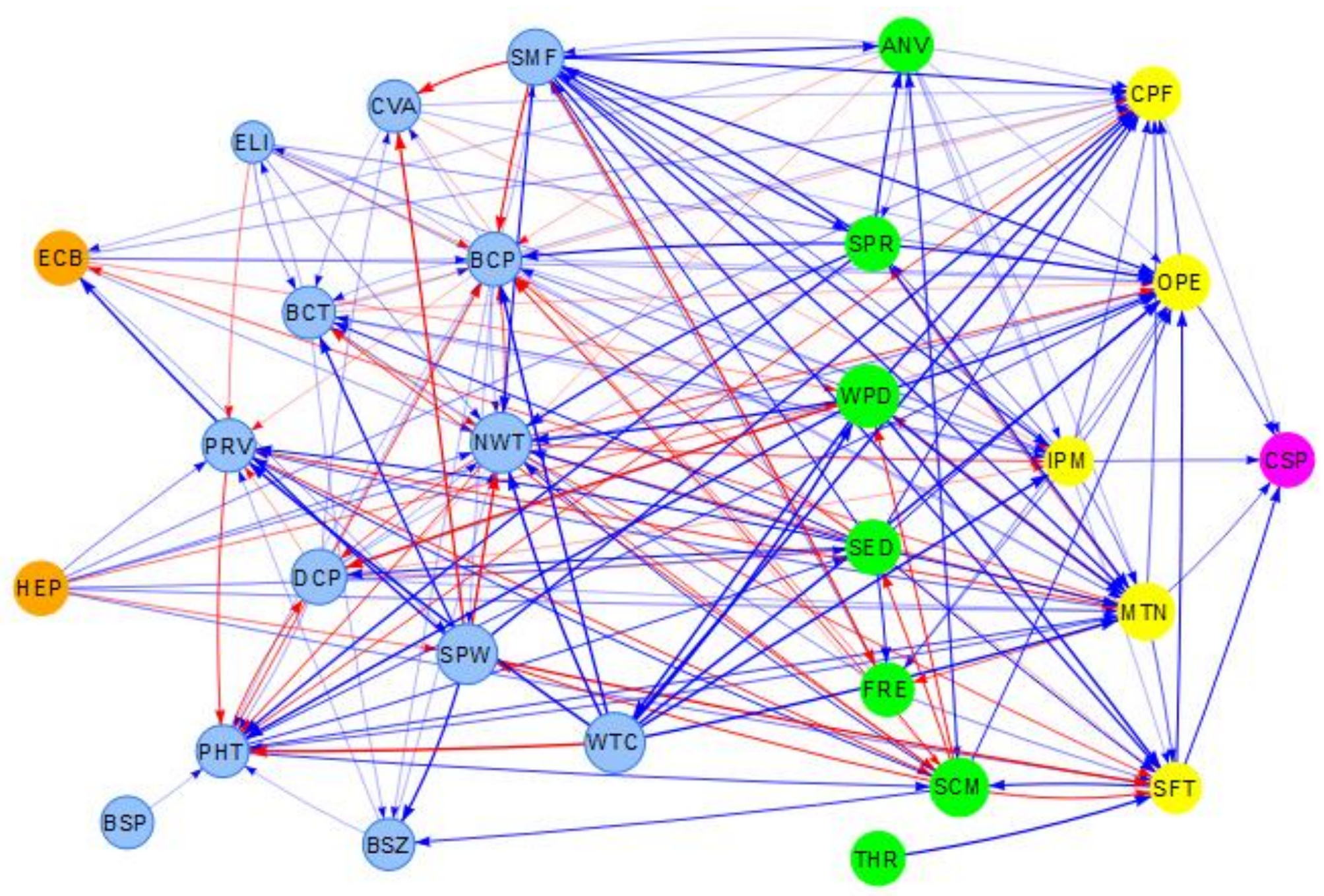

Figure 9.10 PDF Map 
Table 9.11 Adjacency matrix of PDF Map

\begin{tabular}{|c|c|c|c|c|c|c|c|c|c|c|c|c|c|c|c|c|c|c|c|c|c|c|c|c|c|c|c|c|}
\hline & ANV & BCP & BCT & BSP & BSZ & CPF & CSP & CVA & DCP & ECB & ELI & FRE & HEP & IPM & MTN & NWT & OPE & PHT & PRV & SCM & SED & SFT & SMF & SPR & SPW & THR & WPD & WTC \\
\hline ANV & 0 & -0.6 & 0 & & 0 & $\begin{array}{ll}0 & 0.777\end{array}$ & 0 & 0 & 0 & 0.5 & 0 & 0 & & $\begin{array}{ll}0 & 0.537\end{array}$ & 0.41 & 0 & 0.843 & -0.1 & 0 & 0.8 & 0 & 0.68 & 0.65 & 0.44 & 0 & 0 & 0 & 0 \\
\hline BCP & 0 & 0 & 0.843 & & 0 & $\begin{array}{ll}1 & 0.275\end{array}$ & 0 & 1 & 0 & 0 & 0.77 & 0 & & 0.18 & 0.305 & 1 & 0.83 & 1 & -0.62 & 0 & 0 & -0.33 & 0.75 & 0 & 1 & 0 & 0 & 0 \\
\hline BCT & 0 & 0.795 & 0 & & 0 & -0.5 & 0 & 0 & 0 & 0 & 0.89 & 0 & & 1 & 0 & -0.62 & -0.14 & 0 & 0 & 0 & 0 & 0 & 0 & 0 & 0 & 0 & 0 & 0 \\
\hline BSP & 0 & 0 & 0 & & 0 & 0 & 0 & 0 & 0 & 0 & 0 & 0 & & 0 & 0 & 0 & 0 & 1 & 0 & 0 & 0 & 0 & 0 & 0 & 0 & 0 & 0 & 0 \\
\hline BSZ & 0 & 0 & 0 & & 0 & 0 & 0 & 0 & 0 & 0 & 0 & 0 & & 0 & 0 & 1 & 0 & 1 & 0.75 & 0 & 0 & 0 & 0 & 0 & 0 & 0 & 0 & 0 \\
\hline CPF & 0 & 0 & 0 & & 0 & 0 & 0.845 & 0 & 0 & 0 & 0 & 0 & & 0 & 0 & 0 & 0 & 0 & 0 & 0 & 0 & 0 & 0 & 0 & 0 & 0 & 0 & 0 \\
\hline CSP & 0 & 0 & 0 & & 0 & 0 & 0 & 0 & 0 & 0 & 0 & 0 & & 0 & 0 & 0 & 0 & 0 & 0 & 0 & 0 & 0 & 0 & 0 & 0 & 0 & 0 & 0 \\
\hline CVA & 0 & 0 & 0.57 & & 0 & 0.41 & 0 & 0 & 0 & 0 & 0 & 0 & & -0.74 & 0 & 0 & 0.7 & 0 & 0 & -0.82 & 0 & 0 & 0 & 0 & 0 & 0 & 0 & 0 \\
\hline DCP & 0 & 0.285 & 0 & & 0 & $\begin{array}{ll}0 & 0.713\end{array}$ & 0 & 1 & 0 & 0 & 0 & 0 & & -0.58 & 0.897 & 0.732 & 0.895 & 0.4 & -0.02 & 0 & -0.58 & 0 & 0 & 0 & 0 & 0 & 0 & 0 \\
\hline ECB & 0 & 0.69 & 0 & & 0 & 0.741 & 0 & 0 & 0 & 0 & 0 & 0 & & 0 & 0 & 0.08 & 1 & 0 & 0 & 0 & 0 & 0 & 0 & 0 & 0.605 & 0 & -0.7 & 0 \\
\hline ELI & 0 & -0.44 & 0.69 & & 0 & 0 & 0 & 0 & 0 & 0 & 0 & 0 & & 0.46 & 0.18 & 0.37 & 0.51 & 0 & -0.43 & 0 & 0 & 0 & 0 & 0 & 0 & 0 & 0 & 0 \\
\hline FRE & 0 & -0.32 & 0.73 & & 0 & 0 & 0 & 0 & 0 & 0 & 0 & 0 & & 0 & 0 & 0 & 0.1 & 0 & 0 & 0 & 0 & 0.28 & 0.6 & 0 & 0 & 0 & 0 & 0 \\
\hline HEP & 0 & 0 & 0 & & 0 & 0.86 & 0 & 0 & 0 & 0 & 0 & 0 & & 0 & 0.005 & 0.18 & 0.91 & 0 & 0.47 & 0 & 1 & 0.31 & 0 & 0 & -0.89 & 0 & -0.44 & 0 \\
\hline IPM & 0 & 0.18 & 0.453 & & 0 & 0.85 & 0.779 & 0 & 0 & 0 & 0 & 0.82 & & 0 & 0.76 & 0 & 1 & 0 & 0 & 0 & 0 & 0 & 0.75 & 0.88 & 0 & 0 & 0 & 0 \\
\hline MTN & 0 & 0 & 0 & & 0 & 0.69 & 0.759 & 0 & 0 & -1 & 0 & -1 & & 0 & 0 & 0 & 0 & 0.94 & 1 & 0 & 0 & 0.56 & 0 & -0.92 & 0 & 0 & -1 & 0 \\
\hline NWT & 0 & -0.84 & 0 & & 0 & 0 & 0 & 0 & -0.08 & 0 & 0 & 0 & & -0.19 & -0.11 & 0 & -0.27 & -0.79 & 0 & -0.77 & 0 & -0.43 & 0 & 0 & 0 & 0 & 0 & 0 \\
\hline OPE & 0 & 0 & 0 & & 0 & 0 & 0.936 & 0 & 0 & 0 & 0 & 0 & & 0 & 0 & 0 & 0 & 0 & 0 & 0 & 0 & 0 & 0 & 0 & 0 & 0 & 0 & 0 \\
\hline PHT & 0 & -1 & 0 & & 0 & $\begin{array}{ll}0 & -0.34\end{array}$ & 0 & 0 & -1 & 0 & 0 & 0 & & 0 & 1 & 0 & 0.065 & 0 & 0 & 0.6 & 0.75 & 0 & 0 & 0 & 0 & 0 & 0 & 0 \\
\hline PRV & 0 & 0 & 0 & & 0 & 0 & 0 & 0 & 0 & 0 & 0 & 0 & & 0 & -1 & 0 & 0 & -0.77 & 0 & -0.83 & 0 & 0 & 0 & 0 & 0 & 0 & 0 & 0 \\
\hline SCM & 0.48 & -0.51 & -0.37 & & 0 & 1 & 0 & 0 & 0 & 0 & 0 & 0 & & 0 & 0 & 1 & 0.92 & 0 & 0.305 & 0 & -0.44 & -0.66 & 0 & 0 & -0.52 & 0 & -0.07 & 0 \\
\hline SED & 0 & -0.35 & 0.75 & & 0 & 0.87 & 0 & 0 & 0.87 & 0 & 0 & 0.91 & & 0 & 0.676 & 0.52 & 0.92 & 0 & 0.53 & 0 & 0 & 0.925 & 0.44 & 0 & 0 & 0 & 0 & 0 \\
\hline SFT & 0 & 0 & 0 & & 0 & 0 & 0.719 & 0 & 0 & 0 & 0 & 0 & & 0 & 0 & 0 & 1 & 0 & 0 & 0.88 & 0 & 0 & 0 & 0 & 0 & 0 & 0 & 0 \\
\hline SMF & 1 & -0.37 & 0 & & 0 & 0.6 & 0 & -0.3 & 0 & 0 & 0 & -0.02 & & 0.37 & 0.46 & 0.15 & 0.77 & 0 & 0 & 0 & 0 & 0.18 & 0 & 0.53 & 0 & 0 & 0 & 0 \\
\hline SPR & 1 & 0.08 & 0 & & 0 & 0 & 0 & 0 & 0 & 0 & 0 & 0 & & 0 & 0.39 & 0.26 & 0.33 & 0.08 & 0 & 0 & 0 & 0 & 0.63 & 0 & 0 & 0 & 0 & 0 \\
\hline SPW & 0 & 0 & 0.09 & & 0.92 & 0.75 & 0 & -0.52 & 0 & 0.77 & 0 & 0 & & 0 & 0 & -0.89 & 0 & 0 & 0 & 0 & 0 & -0.69 & 0 & 0 & 0 & 0 & 0 & 0 \\
\hline THR & 0 & 0 & 0 & & 0 & 0 & 0 & 0 & 0 & 0 & 0 & 0 & & 0 & 0 & 0 & 0 & 0 & 0 & 0 & 0 & 1 & 0 & 0 & 0 & 0 & 0 & 0 \\
\hline WPD & 0 & 0 & 0 & & 0 & 0.47 & 0 & 0 & -0.3 & 0 & 0 & 0 & & 0 & 0.47 & 0.56 & 0.605 & 0.38 & 0 & 0 & 0 & 0.829 & 0 & 0 & 0 & 0 & 0 & 0.285 \\
\hline WTC & 0 & 0.21 & 0 & & 0 & $\begin{array}{ll}0 & 0.808\end{array}$ & 0 & 0 & 0 & 0 & 0 & 0 & 0 & 0.18 & 1 & 0.835 & 1 & -0.23 & 0.16 & 0 & 0 & 0 & 0 & 0 & 0 & 0 & 0.57 & 0 \\
\hline
\end{tabular}

岁 
Table 9.12 summarizes the structural analysis of PDF Map. PDF Map is comprised of 28 PDFs and 185 relationships so that the corresponding $\mathrm{C} / \mathrm{N}$ value is 6.607 , and the density is 0.245 . Furthermore, it includes three drivers and only one receiver (CSP), resulting in 0.333 of the complexity score.

Table 9.12 Structural analysis of PDF Map

\begin{tabular}{rr} 
& PDF Map \\
\hline Number of PDFs & 28 \\
Number of Relationships & 185 \\
C/N Value & 6.607 \\
Density & 0.245 \\
Number of Drivers & 3 \\
Number of Receivers & 1 \\
Number of Ordinaries & 24 \\
Complexity Score & 0.333 \\
\hline
\end{tabular}

Figure 9.11 summarizes the centralities of each PDF in PDF Map. The FCM has relatively high in-degree centralities of OPE (12.81) and CPF (10.66) while it has high out-degree centrality of BCP (9.90), MTN (7.87) and SED (7.76). Consequently, BCP, MTN, and OPE have high degrees of centrality $(16.56,15.54$, and 14.75 correspondingly) in PDF Map. 


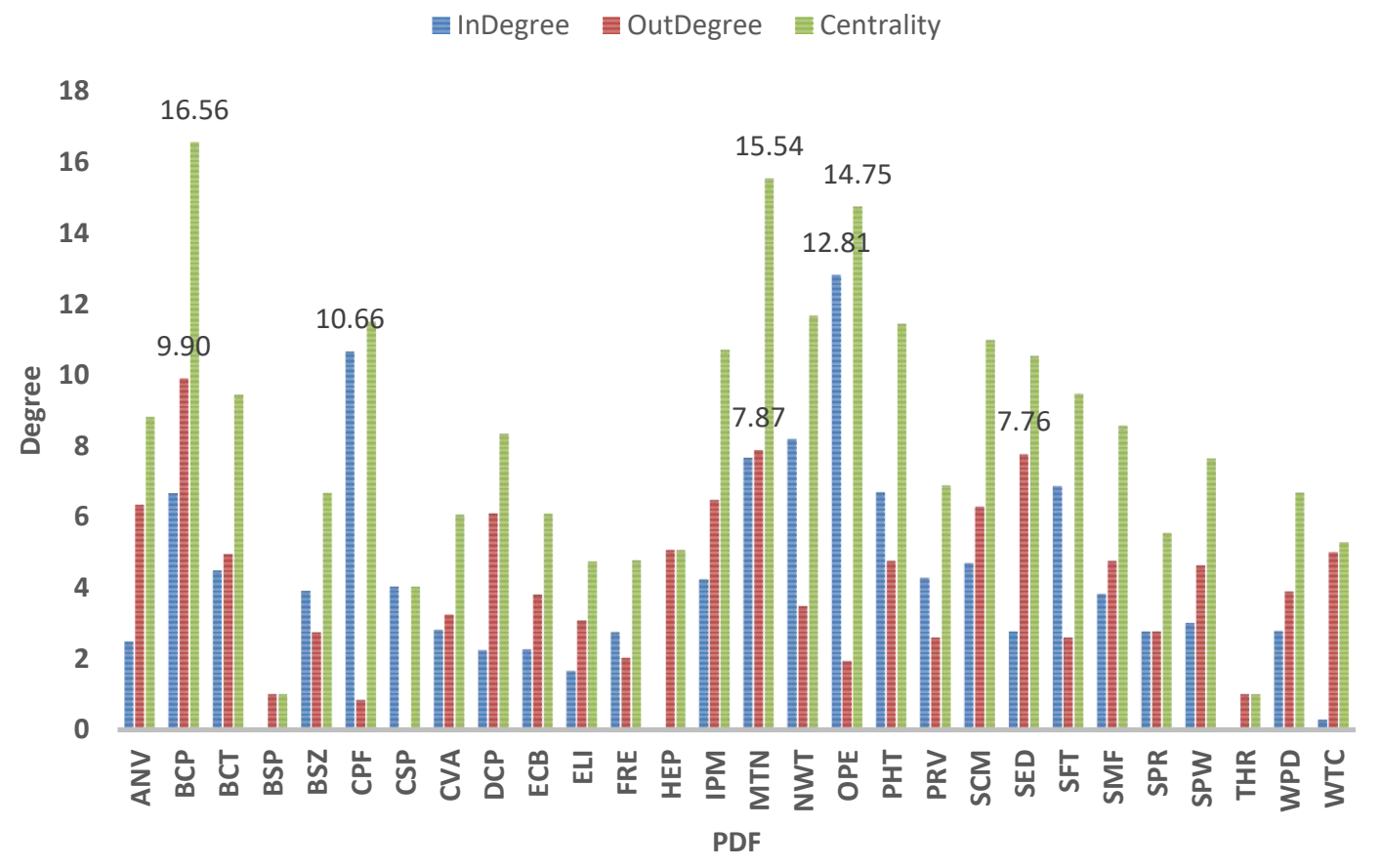

Figure 9.11 Centrality analysis of PDF Map

The responses of the six upper-level PDFs to the activation of BCP were simulated to investigate the dynamic behavior characteristics of PDF Map. Figure 9.12 shows the simulation result about the dynamic behavior of PDF Map, and Table 9.13 summarizes the settled response value of the six upper-level PDFs and converged iteration number of the stimulation.

The activation of BCP resulted in the very positive value of CPF (1.000), OPE (1.000), IPM (1.000), MTN (9.998) and CSP (1.000), whereas SFT was very negative at -0.982 . The response of simulation converged after the $39^{\text {th }}$ iteration, meaning that the value of SFT settled down more slowly than that of other factors. This dynamic behavior is similar to the Tech Map. 


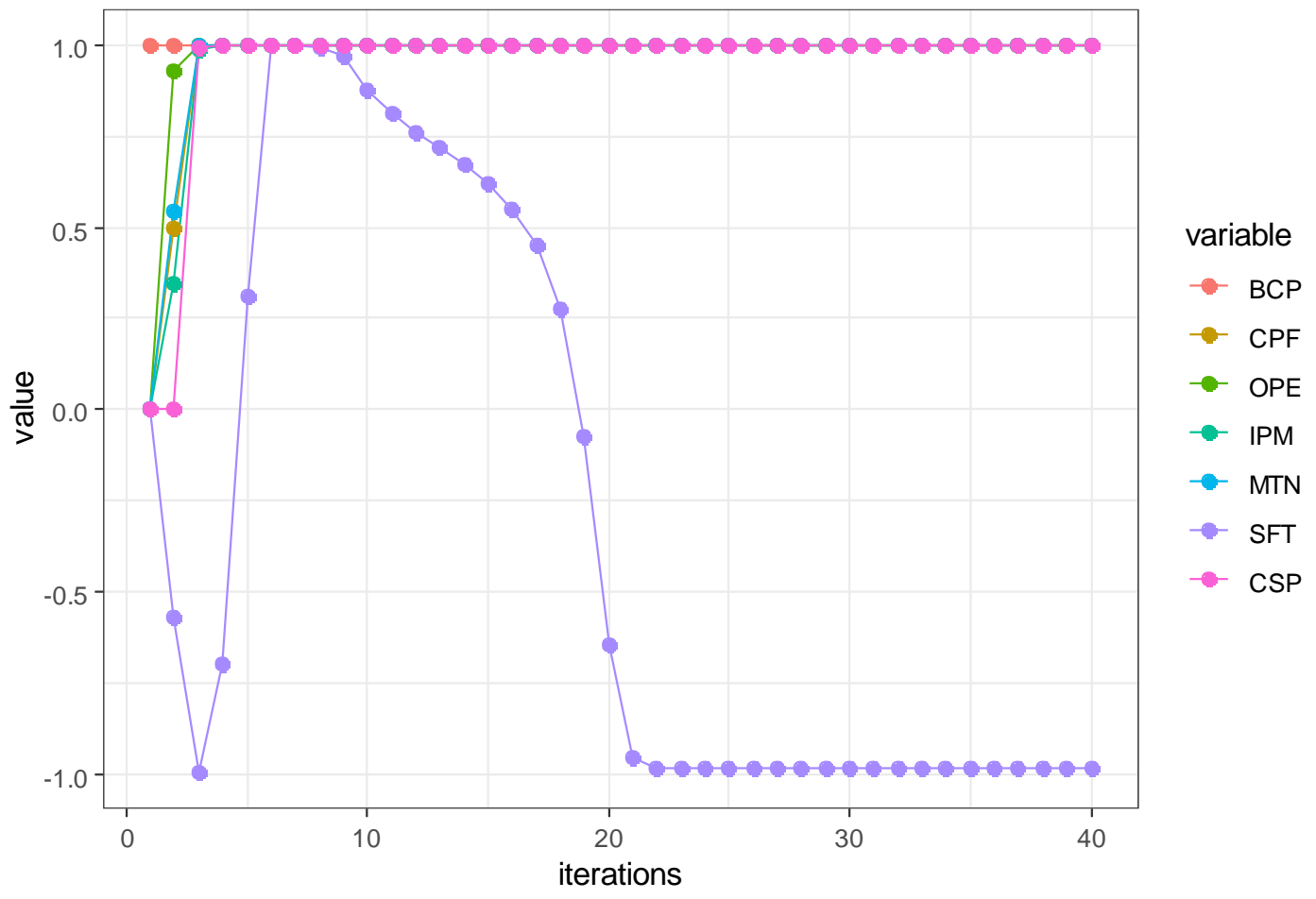

Figure 9.12 Dynamic behavior of PDF Map

Table 9.13 Settled responses \& converged iteration number in PDF Map

\begin{tabular}{ccccccccc} 
& BCP & CPF & OPE & IPM & MTN & SFT & CSP & $\begin{array}{l}\text { Converged } \\
\text { Iteration Number }\end{array}$ \\
\hline PDF Map & 1.000 & 1.000 & 1.000 & 1.000 & 0.998 & -0.982 & 1.000 & 39 \\
\hline
\end{tabular}

\subsubsection{Scenarios - Product Concepts}

During the workshop, the researcher asked each participant to select three fundamental PDFs which he/she regarded more highly effective ones to CSP than the others. Consequently, participants selected four fundamental PDFs: BCP, BCT, ANV, and PHT. Subsequently, the researcher facilitated a discussion about what the participants expected of desirable UniCleanBot's characteristics based on the four PDFs and the product design requirement document shared with participants before the workshop. The discussion produced three desirable scenarios: 
Firstly, the group of participants pointed out the battery performance of UniCleanBot. Associated with the battery performance, the participants emphasized the importance of battery performance in enhancing cleaning performance and intelligent power management. The battery performance is characterized by its capacity and charging time. Higher battery capacity provides more and longer electric energy, which enables to increase the suction power, mobility performance, or the cleaning time with one charge. Additionally, faster battery charging time benefits in shortening the cleaning cycle. For this scenario, the first simulation carried with the activations of BCP and BCT.

Secondly, the group suggested that the developing robotic floor cleaner is required to provide operational convenience to its users. Considering the benefits a robotic device brings, it is important to minimize any user intervention in its operation. In this regard, an advanced navigation system enhances cleaning performance by optimizing a pathway of moving, which may minimize a user's intervention. In addition to the advanced navigation system, shorter battery charging time enables UniCleanBot to complete the cleaning work in less time. Accordingly, an owner of UniCleanBot can reduce any burdens caused by its noise or collision during cleaning works. Consequently, ANV and $\mathrm{BCT}$ were used as activators for the second simulation.

Lastly, the group concluded that the cleaning coverage is critical for the customer preference of UniCleanBot. The design elements to increase cleaning coverage of UniCleanBot are low profile and high battery capacity. During cleaning, UniCleanBot will wander around every corner at home. From time to time, its cleaning coverage is limited because of the height of UniCleanBot. For instance, when the height of 
UniCleanBot is not low enough, it may be stuck under a bed or unable to access there. In this case, the cleaning coverage will be limited. Therefore, the low profile height enables UniCleanBot to widen its cleaning coverage. Furthermore, as aforementioned, high battery capacity benefits to widen the cleaning coverage of UniCleanBot by providing more energy for its operation. The third scenario was simulated by activating PHT and BCP.

In addition to the three scenarios discussed above, the last scenario was simulated under the assumption that customers would value all product variations represented in cases 1-3 and therefore combined all prior scenarios.

Table 9.14 summarizes which product design factors are activated for each scenario in simulation.

\section{Table 9.14 Activation of product design factors for each scenario}

\begin{tabular}{|c|c|c|c|c|c|}
\hline \multirow{2}{*}{ Scenario No. } & \multirow{2}{*}{ Description } & \multicolumn{4}{|c|}{ Activation of Product Design Factor } \\
\hline & & $\mathrm{BCP}$ & BCT & ANV & PHT \\
\hline Scenario 1 & $\begin{array}{l}\text { Customers prioritize the battery } \\
\text { performance. }\end{array}$ & $\checkmark$ & $\checkmark$ & & \\
\hline Scenario 2 & $\begin{array}{l}\text { Customers prioritize the } \\
\text { operational convenience. }\end{array}$ & & $\checkmark$ & $\checkmark$ & \\
\hline Scenario 3 & $\begin{array}{l}\text { Customers prioritize the } \\
\text { cleaning coverage. }\end{array}$ & $\checkmark$ & & & $\checkmark$ \\
\hline Scenario 4 & $\begin{array}{l}\text { Customers value all cases } \\
\text { above. }\end{array}$ & $\checkmark$ & $\checkmark$ & $\checkmark$ & $\checkmark$ \\
\hline
\end{tabular}

\subsubsection{Simulations}

This section provides a description of the simulation results carried out with the PDF map based on the scenarios developed in the previous section. In order to simulate all four scenarios, commonly, a hyperbolic tangent function $(\lambda=0.5)$ described in 
Equation (6) was applied as the squashing function, outputting a CSP value between -1 and 1 with the other five upper-level factors (CPF, OPE, IPM, MTN and SFT) as the result of each simulation. (The CSP value is regarded as the CPV, the result value of CDRM.) The iteration of each simulation was continued until the error difference among each subsequent PDF diminishes less than 0.00001 as the minimum error difference. If the error difference does not reach the minimum value, the iteration number was adjusted.

\section{Scenario 1 - Prioritization of Battery Performance}

The first scenario assumes that customers prioritize the battery performance of UniCleanBot. For this scenario, the battery capacity (BCP) and the battery charging time (BCT) were activated in the initial state vector. Therefore, for the initial state vector in the simulation, $\mathrm{BCP}$ and $\mathrm{BCT}$ have the highest value (1) while the others set as zero.

Figure 9.13 and Table 9.15 show the dynamic behaviors of the simulation results and the result values of the upper-level PDFs for Scenario 1. The dynamic behaviors of the results are settled after the $42^{\text {nd }}$ iteration. The CSP value is 0.971 , which means that the customer preference is high relatively when Battery Performance is prioritized in engineering design. 


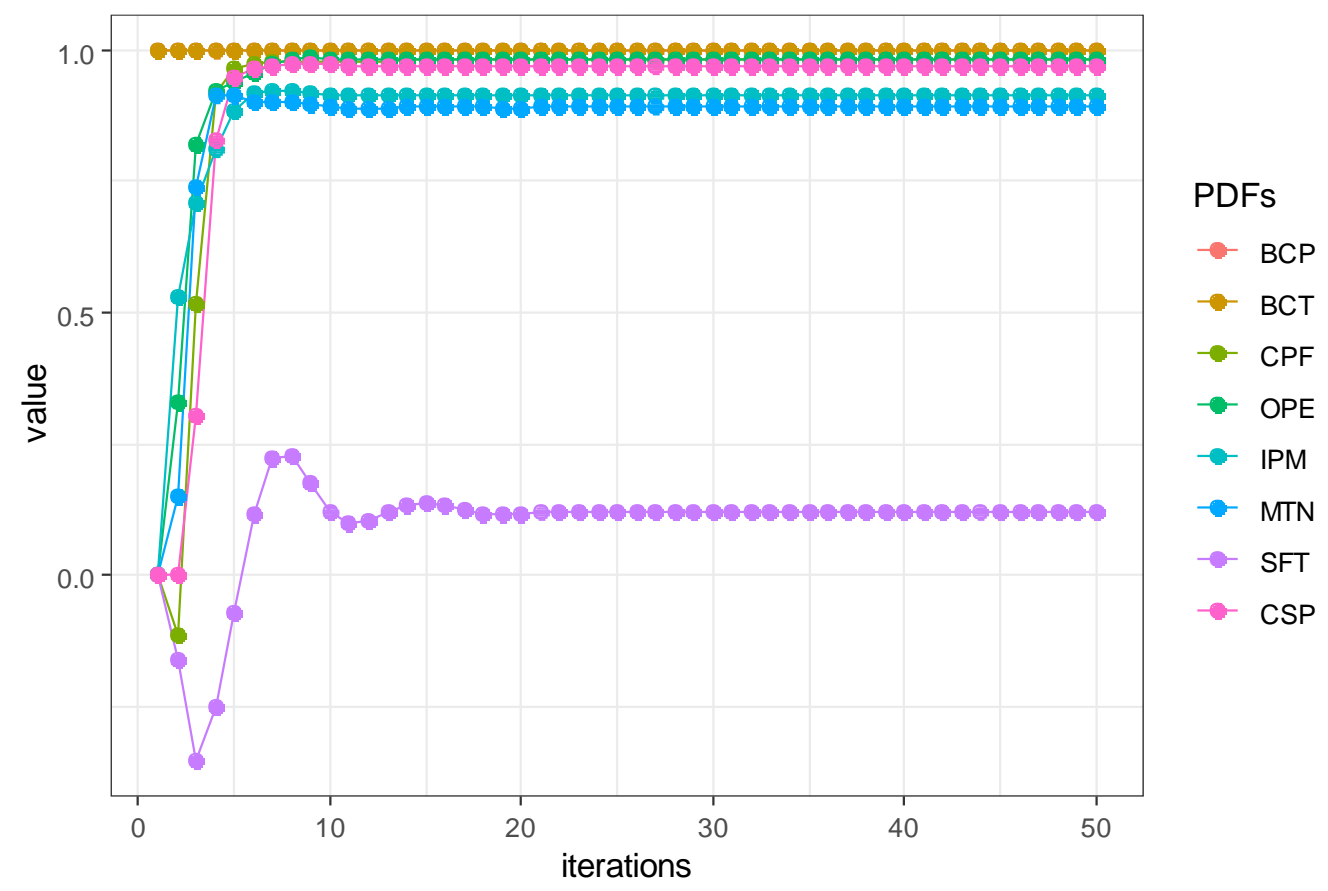

Figure 9.13 Dynamic behaviors of simulation results for Scenario 1

Table 9.15 Result values of the upper-level PDFs for Scenario 1

\begin{tabular}{ccccccccccc} 
& BCP & BCT & CPF & OPE & IPM & MTN & SFT & CSP & $\begin{array}{l}\text { Converged } \\
\text { lteration Number }\end{array}$ \\
\hline Scenario 1 & 1.000 & 1.000 & 0.980 & 0.981 & 0.913 & 0.890 & 0.121 & $\mathbf{0 . 9 7 0}$ & 42 \\
\hline
\end{tabular}

\section{Scenario 2 - Prioritization of Operational Convenience}

The second scenario directs the assumption that engineering design prioritizes the operational convenience of UniCleanBot for customer preference. For this scenario, the initial vector is formed with the activation of the high-performance navigation function and of the battery charging time.

Figure 9.14 shows the dynamic behaviors of the simulation results for Scenario 2. As shown, the resulting values of the six upper-level PDFs are not settled but fluctuated continuously. Though the maximum iteration option changed to 500 , the resulting values 
were not converged. Consequently, it is difficult to draw a meaningful result from this scenario.

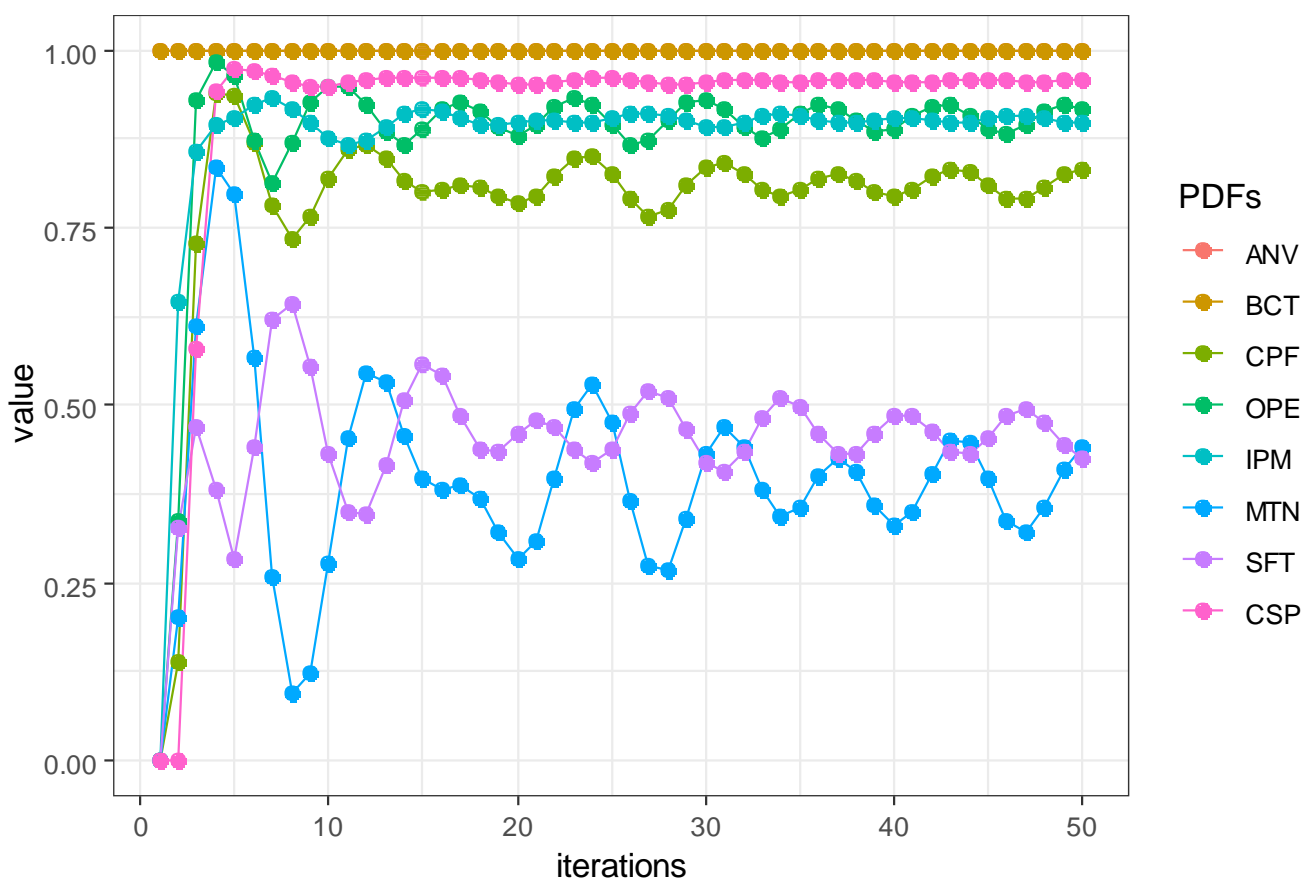

Figure 9.14 Dynamic behaviors of simulation results for Scenario 2

\section{Scenario 3 - Prioritization of Cleaning Coverage}

The third scenario was simulated to test the effect on customer preference when engineering design emphasizes the cleaning coverage of UniCleanBot. The initial state vector includes two active PDFs, BCP and PHT.

Figure 9.15 shows the dynamic behaviors of the simulation results, and Table 9.16 summarizes the result values of the upper-level PDFs for Scenario 3. All results are settled down after the $39^{\text {th }}$ iteration. The settled CSP value is 0.971 , which means that the customer preference is slightly higher than Scenario 1 when Cleaning Coverage is prioritized in engineering design. 


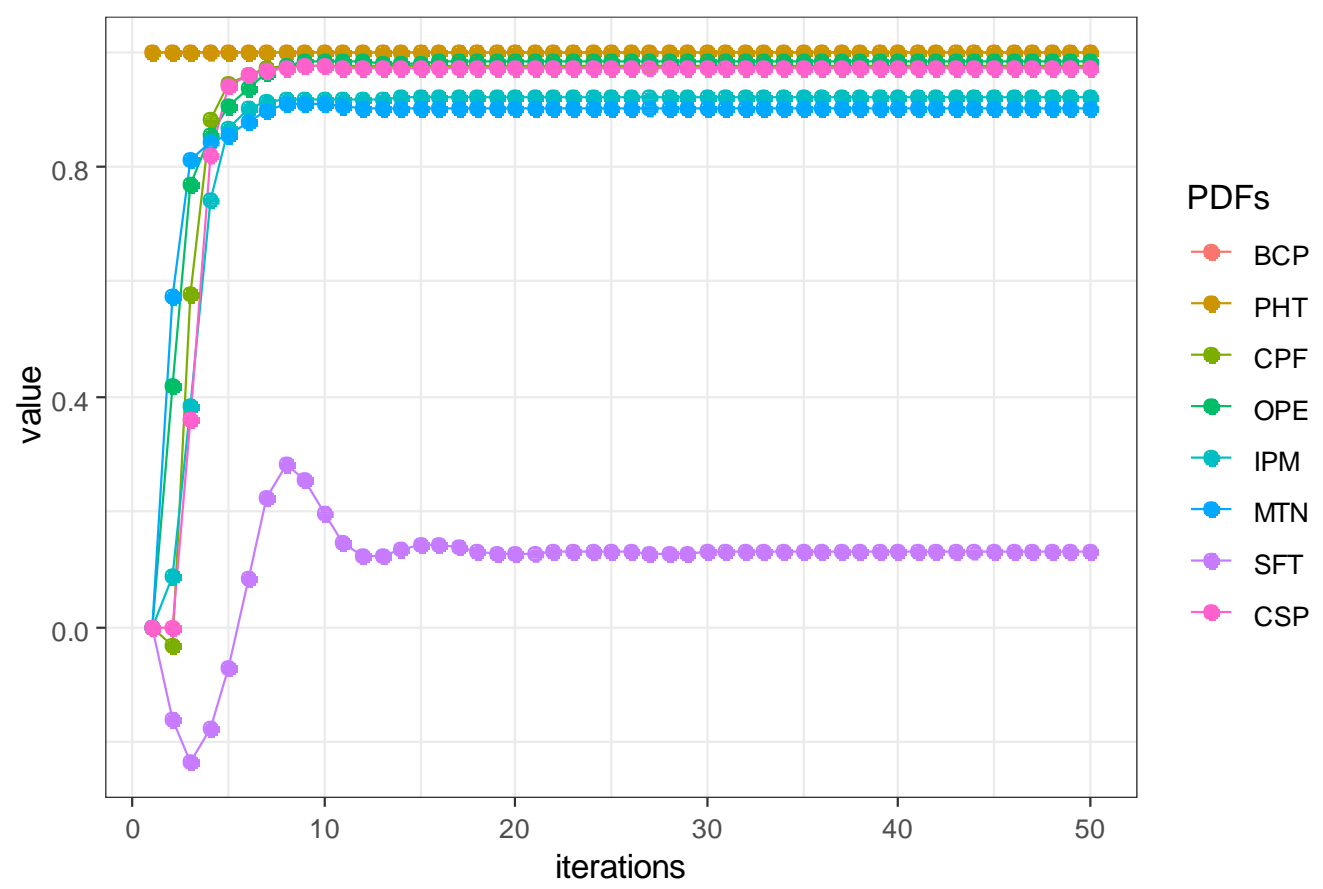

Figure 9.15 Dynamic behaviors of simulation results for Scenario 3

Table 9.16 Result values of the upper-level PDFs for Scenario 3

\begin{tabular}{cccccccccccc} 
& BCP & PHT & \multicolumn{2}{c}{ CPF } & OPE & IPM & MTN & SFT & CSP & \multicolumn{2}{l}{$\begin{array}{l}\text { Converged } \\
\text { Iteration Number }\end{array}$} \\
\hline Scenario 3 & 1.000 & 1.000 & 0.977 & 0.982 & 0.920 & 0.902 & 0.129 & $\mathbf{0 . 9 7 1}$ & 39 \\
\hline
\end{tabular}

\section{Scenario 4 - Consideration of All Cases}

The last scenario describes the assumption that customers value all scenarios aforementioned. Therefore, all four fundamental PDFs the participants selected are activated as the union set of the initial vectors in the initial state vector.

Figure 9.16 and Table 9.17 show the dynamic behaviors of simulation results and the result values of the upper-level PDFs for Scenario 4. All results are converged after the $36^{\text {th }}$ iteration, and the converged CSP value is 0.971 , which means that the customer preference is the same as Scenario 3 when all previous cases are considered equally in engineering design. 


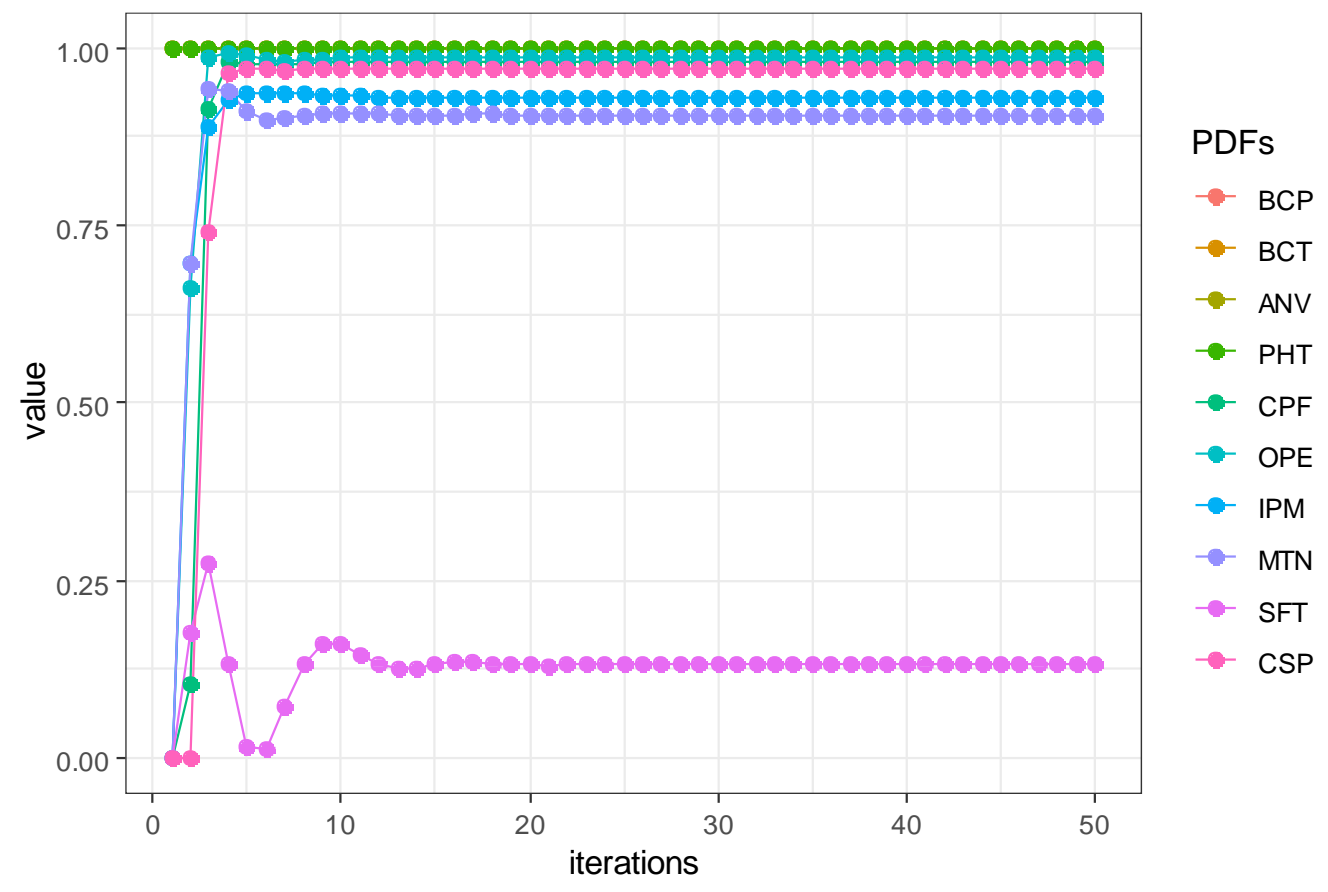

Figure 9.16 Dynamic behaviors of simulation results for Scenario 4

Table 9.17 Result values of the upper-level PDFs for Scenario 4

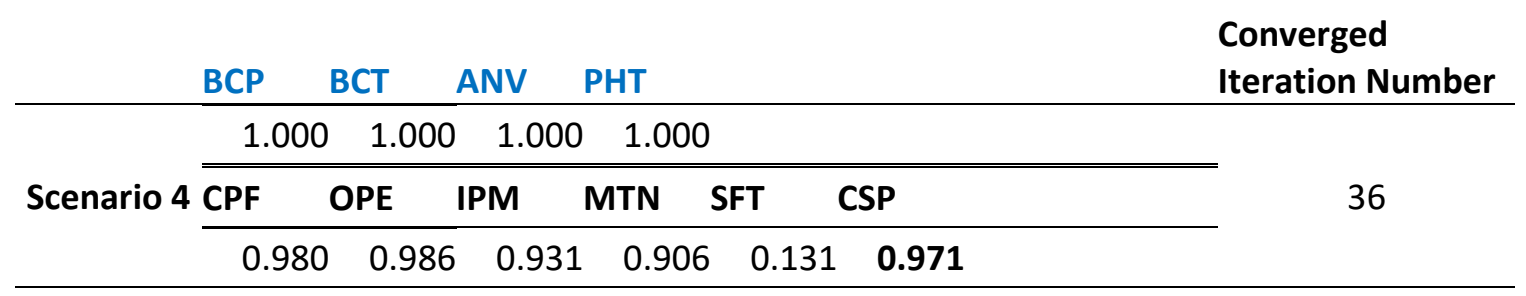

\section{Summary}

Table 9.18 summarizes all simulation results for Scenario 1 to 4 . In Table 9.18, the second scenario does not have any resulting values because the results were not converged. Except for Scenario 2, the other three scenarios resulted in positive CSP values, which means that these scenarios benefit the customer preference of UniCleanBot. Among the three scenarios, the fourth scenario produced the highest CSP value $(0.971)$ without any negative values of the five benefit PDFs compared with Scenario 1 and Scenario 3. On the other hand, the first scenario resulted in the lowest CSP value (0.970). 
As a result, according to the CDRM process, participants of this experiment as an engineering design team can recommend the last scenario, which resulted in the highest values of all upper-level PDFs. However, there are not distinctive differences among the resulting CSP values of the three scenarios (Scenario 1, Scenario 3, and Scenario 4). Nevertheless, comparing the benefit PDFs (CPF, OPE, IPM, MTN, and SFT), the last scenario can be the best option for UniCleanBot.

\section{Table 9.18 Summary of simulation results}

\begin{tabular}{|c|c|c|c|c|c|c|c|c|c|c|c|}
\hline & \multicolumn{4}{|c|}{$\begin{array}{l}\text { Activating Value of } \\
\text { Fundamental PDFs }\end{array}$} & \multicolumn{6}{|c|}{ Resulting Values of the Simulations } & \multirow{2}{*}{$\begin{array}{l}\text { Converged } \\
\text { Iteration } \\
\text { Number }\end{array}$} \\
\hline & $\mathrm{BCP}$ & BCT & ANV & PHT & CPF & OPE & IPM & MTN & SFT & CSP & \\
\hline Scenario 1 & 1.000 & 1.000 & - & - & 0.980 & 0.981 & 0.913 & 30.890 & 0.121 & 10.970 & 42 \\
\hline Scenario 2 & - & 1.000 & 1.000 & - & - & - & - & - & - & - & - \\
\hline Scenario 3 & 1.000 & - & - & 1.000 & 0.977 & 0.982 & 0.920 & 0.902 & 0.129 & 90.971 & 39 \\
\hline Scenario 4 & 1.000 & 1.000 & 1.000 & 1.000 & 0.980 & 0.986 & 0.931 & 0.906 & 0.131 & 10.971 & 36 \\
\hline
\end{tabular}

\subsubsection{Revising Model Structure}

In conducting the simulations in the previous subsections, some simulation results presented "Not Converged" states, which can be regarded as a limit cycle or chaotic system behavior. According to Kosko [210], this phenomenon frequently occurs when a fuzzy cognitive map includes feedback loops among concepts. For instance, in this research, E5's and E8's FCMs have one or more feedback loop. As a result, PDF Map aggregated with eight individual FCMs have several feedback loops, which causes nonlinear dynamic behavior of the responses in simulation. Non-convergence makes it impossible to compare the stable end state of alternative product concepts (i.e., different input scenarios) and to draw conclusions about customer preference in CDRM. Therefore, when CDRM is applied in a real project, the possibility that a PDF Map has feedback 
loops needs to be considered carefully: the product development team needs to discuss whether the feedback loops are real, avoidable, and should be included in the model.

Furthermore, as mentioned in Subsection 7.3.6, some participants were confused about when and how to connect concepts during FCM development, possibly because of insufficient instructions before during the workshop. Because of the confusion, the weights of some relationships have signs that appear the opposite of what the participant probably wanted to express. These wrong assignments were not dealt with during the discussion and the relationships have remained in PDF Map without any modifications, which affected the simulation results.

I used my observations about feedback loops and edge signs for the development of criteria for model refinement,. I applied them to revise the model structure of PDF Map in order to achieve simulation results that converge. For this revision, the adjacency matrix of PDF Map was reviewed focusing on whether a feedback loop is connected logically and whether any weights on relationships have an opposite sign from the viewpoint of engineering design. If any feedback loops are linked illogically, one of two relationships forming a feedback loop was disconnected. Any weights having an opposite sign were also corrected considering the rule of a relationship assignment in FCM development. As a result, 63 relationships were disconnected, and 10 changed the signs, which led to no feedback loop in the new PDF Map. In total, the number of relationships decreased by around 34\%. Figure 9.17 and Table 9.19 are the restructured PDF Map (RPDF Map) and its adjacency matrix correspondingly. 


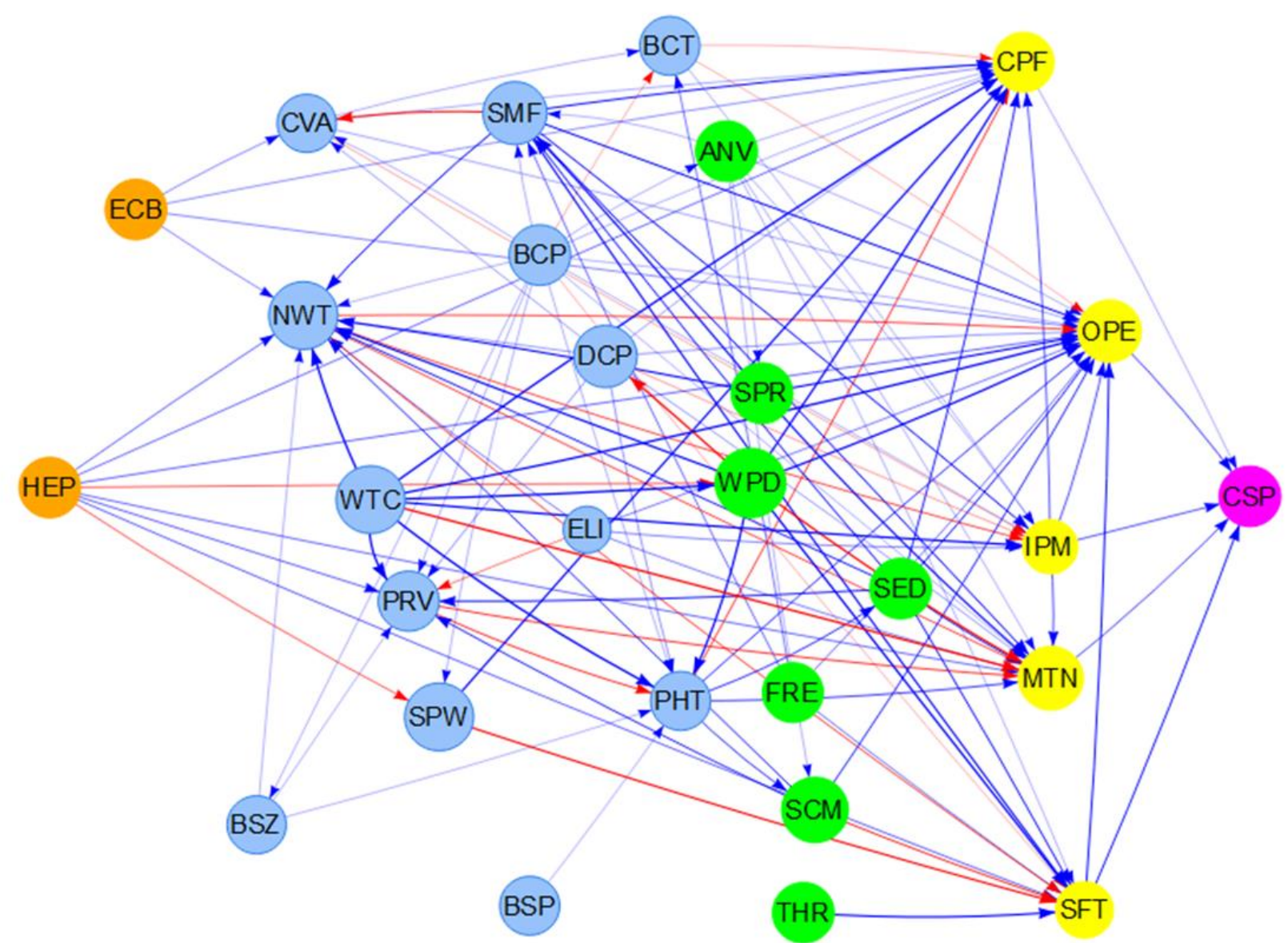


Table 9.19 Adjacency matrix of RPDF Map

\begin{tabular}{|c|c|c|c|c|c|c|c|c|c|c|c|c|c|c|c|c|c|c|c|c|c|c|c|c|c|c|c|c|}
\hline & ANV & BCP & BCT & BSP & BSZ & CPF & CSP & CVA & DCP & ECB & ELI & FRE & HEP & IPM & MTN & NWT & OPE & PHT & PRV & SCM & SED & SFT & SMF & SPR & SPW & THR & WPD & WTC \\
\hline ANV & & & & & & 0.78 & & & & & & & & 0.54 & 0.41 & & 0.84 & & & 0.8 & & 0.68 & 0.65 & 0.44 & & & & \\
\hline BCP & 0.6 & & -0.8 & & 1 & 0.28 & & 1 & & & & & & 1 & 0.31 & 1 & 0.83 & 1 & 0.62 & & & -0.3 & 0.75 & & 1 & 1 & & \\
\hline BCT & & & & & & -0.5 & & & & & & & & 1 & & & -0.1 & & & & & & & & & & & \\
\hline BSP & & & & & & & & & & & & & & & & & & 1 & & & & & & & & & & \\
\hline BSZ & & & & & & & & & & & & & & & & 1 & & 1 & 0.75 & & & & & & & & & \\
\hline CPF & & & & & & & 0.85 & & & & & & & & & & & & & & & & & & & & & \\
\hline CSP & & & & & & & & & & & & & & & & & & & & & & & & & & & & \\
\hline CVA & & & 0.57 & & & 0.41 & & & & & & & & -0.7 & & & 0.7 & & & & & & & & & & & \\
\hline DCP & & & & & & 0.71 & & 1 & & & & & & -0.6 & 0.9 & 0.73 & 0.9 & 0.4 & 0.02 & & & & & & & & & \\
\hline ECB & & & & & & 0.74 & & 0.2 & & & & & & & & 0.08 & 1 & & & & & & & & & & & \\
\hline ELI & & & & & & & & & & & & & & 0.46 & 0.18 & 0.37 & 0.51 & & -0.4 & & & & & & & & & \\
\hline FRE & & & 0.73 & & & & & & & & & & & & & & 0.1 & & & & & 0.28 & 0.6 & & & & & \\
\hline HEP & & & & & & 0.86 & & & & & & & & & 0.01 & 0.18 & 0.91 & & 0.47 & & & 0.31 & & & -0.9 & & -0.4 & \\
\hline IPM & & & & & & 0.85 & 0.78 & & & & & & & & 0.76 & & 1 & & & & & & & & & & & \\
\hline MTN & & & & & & & 0.76 & & & & & & & & & & & & & & & & & & & & & \\
\hline NWT & & & & & & & & & & & & & & -0.2 & -0.1 & & -0.3 & & & & & -0.4 & & & & & & \\
\hline OPE & & & & & & & 0.94 & & & & & & & & & & & & & & & & & & & & & \\
\hline PHT & & & & & & -0.3 & & & & & & & & & 1 & & 0.07 & & & 0.6 & 0.75 & & & & & & & \\
\hline PRV & & & & & & & & & & & & & & & -1 & & & -0.8 & & & & & & & & & & \\
\hline SCM & & & & & & & & & & & & & & & & 1 & 0.92 & & 0.31 & & & -0.7 & & & & & & \\
\hline SED & & & & & & 0.87 & & & & & & & & & 0.68 & 0.52 & 0.92 & & 0.53 & & & 0.92 & 0.44 & & & & & \\
\hline SFT & & & & & & & 0.72 & & & & & & & & & & 1 & & & & & & & & & & & \\
\hline SMF & & & & & & 0.6 & & -0.3 & & & & & & 0.37 & 0.46 & 0.15 & 0.77 & & & & & 0.18 & & & & & & \\
\hline SPR & & & & & & & & & & & & & & & 0.39 & 0.26 & 0.33 & & & & & & 0.63 & & & & & \\
\hline SPW & & & & & & 0.75 & & & & & & & & & & & & & & & & -0.7 & & & & & & \\
\hline THR & & & & & & & & & & & & & & & & & & & & & & 1 & & & & & & \\
\hline WPD & & & & & & 0.47 & & & -0.3 & & & & & & -0.5 & 0.56 & 0.61 & 0.38 & & & & 0.83 & & & & & & \\
\hline WTC & & & & & & 0.81 & & & & & & & & 0.18 & -1 & 0.84 & 1 & 0.23 & 0.16 & & & & & & & & 0.57 & \\
\hline
\end{tabular}


Because of the reduced number of the relationship, RPDF Map has lower density compared to the original PDF Map as shown in Table 9.20. In addition, 5 PDFs (BCP, ECB, ELI, FRE, and WTC) changed from ordinary concepts to driver ones. Consequently, the density of RPDF Map is also lower than the original PDF Map.

\section{Table 9.20 Structural analysis of RPDF Map}

\begin{tabular}{rrr} 
& PDF Map & $\begin{array}{c}\text { Restructured } \\
\text { PDF Map }\end{array}$ \\
\hline Number of PDFs & 28 & 28 \\
Number of Relationships & 185 & 122 \\
C/N Value & 6.607 & 4.357 \\
Density & 0.245 & 0.161 \\
Number of Drivers & 3 & 8 \\
Number of Receivers & 1 & 1 \\
Number of Ordinaries & 24 & 19 \\
Complexity Score & 0.333 & 0.125 \\
\hline
\end{tabular}

Figure 9.18 presents the centralities of each PDF in RPDF Map. OPE has the highest degree of centrality and In-degree centrality. BCP having the highest degree of centrality in the original PDF Map has the second-highest degree of centrality and the highest Out-degree centrality while no In-degree centrality. After revising, BCP still affects other PDFs highly. 


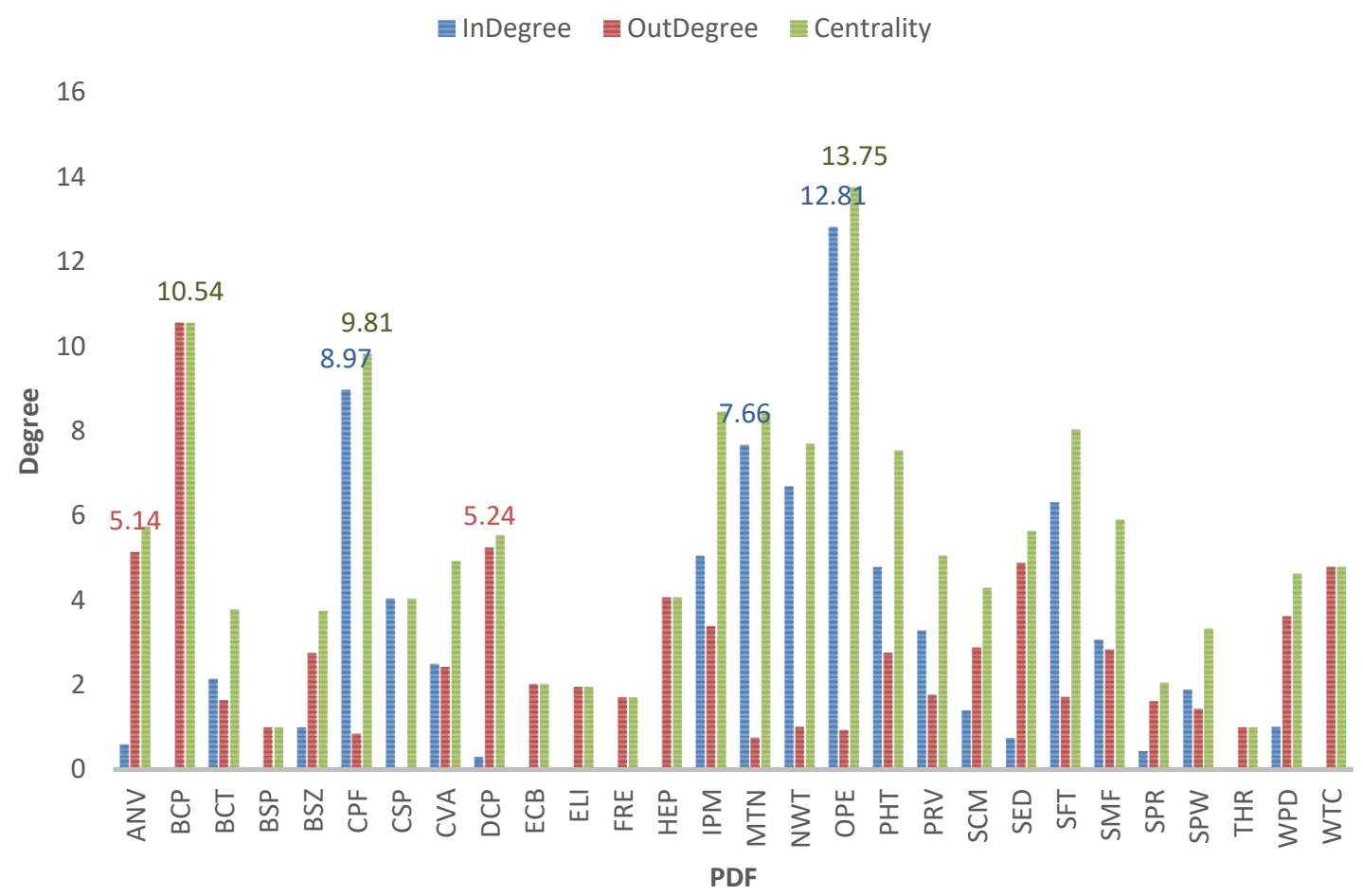

Figure 9.18 Centrality analysis of RPDF Map

In order to analyze the dynamic behavior of RPDF Map, the same simulation was carried out with activating BCP. Figure 9.19 depicts the responses of the six upper-level PDFs, and Table 9.21 summarizes their converged value. Compared to the dynamic behavior of the original PDF Map shown in Figure 9.12, all responses of RPDF Map are converged much earlier (at the $8^{\text {th }}$ iteration), resulted from getting rid of the feedback loops. The resulting value of IPM decreased slightly (1 to 0.955), and the SFT value has a negative value similar to one in the original PDF Map. This implies that high battery capacity impacts negatively on the safety of UniCleanBot, as it did in the original PDF Map. 


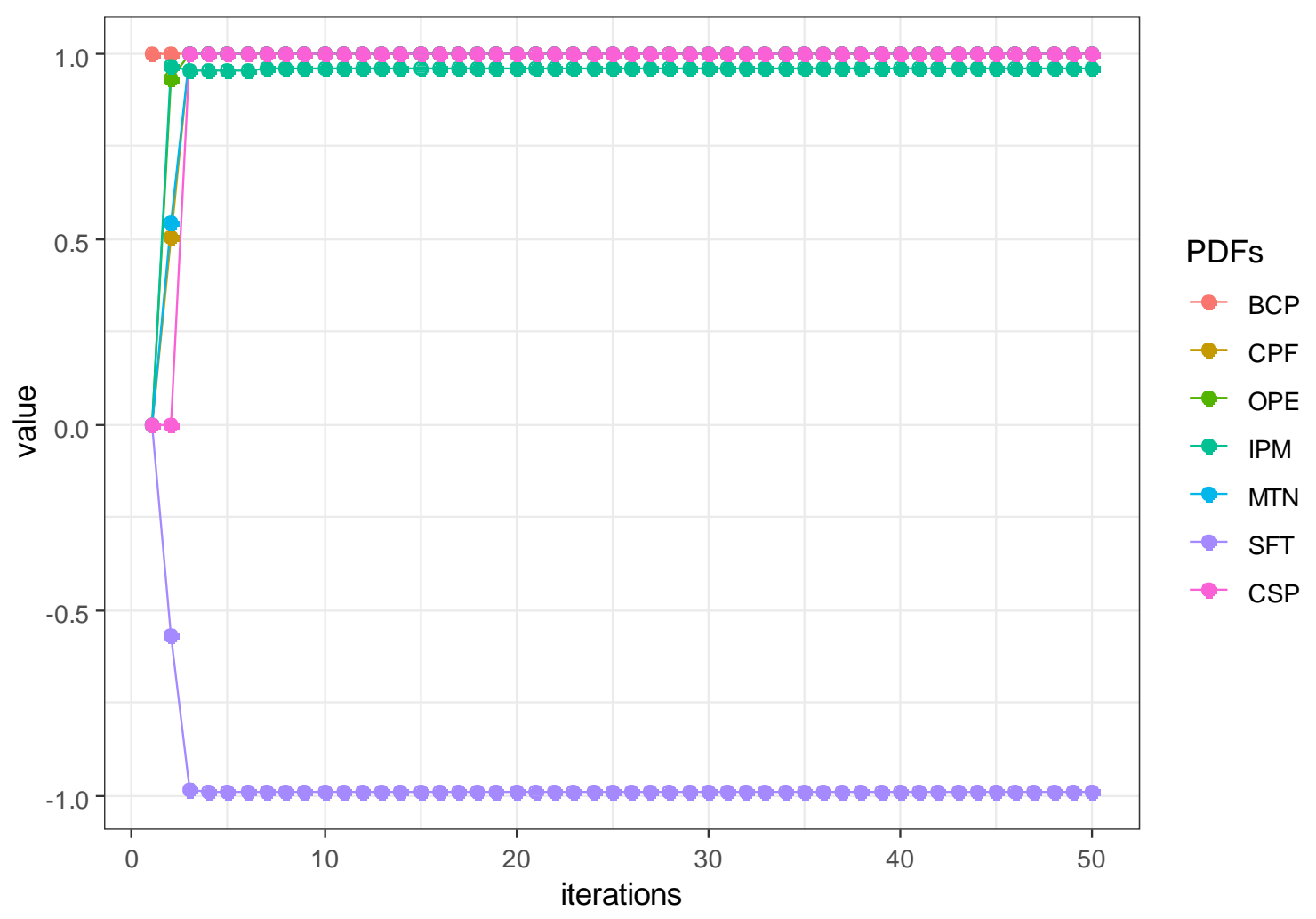

Figure 9.19 Dynamic behavior of RPDF Map

Table 9.21 Settled responses \& converged iteration number in PDF and RPDF Maps

\begin{tabular}{ccccccccc} 
& BCP & CPF & OPE & IPM & MTN & SFT & CSP & $\begin{array}{l}\text { Converged } \\
\text { Iteration Number }\end{array}$ \\
\hline PDF Map & 1.000 & 1.000 & 1.000 & 1.000 & 0.998 & -0.982 & 1.000 & 39 \\
RPDF Map & 1.000 & 1.000 & 1.000 & 0.955 & 1.000 & -0.990 & 1.000 & 8 \\
\hline
\end{tabular}

Next, with RPDF Map, it is necessary to confirm whether the simulation results change under the same setting used with the original PDF Map. Table 9.22 summarizes the simulation results produced by simulating RPDF Map with four alternative input scenarios. Unlike the simulation results in the previous subsection, the simulation results for Scenario 2 output converged results. In addition, all results for the four scenarios were converged within $32^{\text {nd }}$ iteration, which reflects that the structure of RPDF Map is less complicated than the original one. 
Comparing the CSP values in the four simulation results, Scenario 4 also resulted in the highest CSP value like the previous simulation results with the original PDF Map. However, three alternative input scenarios besides Scenario 2 led to negative values of SFT. Therefore, considering all six upper-level PDF values, Scenario 2 is the best alternative input scenario in terms of customer value in spite of that the CSP value for Scenario 4 is the highest.

Table 9.22 Summary of simulation results with RPDF Map

\begin{tabular}{|c|c|c|c|c|c|c|c|c|c|c|c|}
\hline & \multicolumn{4}{|c|}{$\begin{array}{l}\text { Activating Value of } \\
\text { Fundamental PDFs }\end{array}$} & \multicolumn{6}{|c|}{ Resulting Values of the Simulations } & \multirow{2}{*}{$\begin{array}{l}\text { Converged } \\
\text { Iteration } \\
\text { Number }\end{array}$} \\
\hline & BCP & $\mathrm{BCT}$ & ANV & PHT & CPF & OPE & IPM & MTN & SFT & CSP & \\
\hline Scenario 1 & 1.000 & 1.000 & - & - & 0.924 & 0.973 & 30.889 & 0.858 & $8-0.56$ & 20.946 & 18 \\
\hline Scenario 2 & - & 1.000 & 1.000 & - & 0.775 & 0.935 & $5 \quad 0.871$ & 0.742 & 20.09 & 50.956 & 32 \\
\hline Scenario 3 & 1.000 & - & - & 1.000 & 0.948 & 0.973 & 0.444 & 0.862 & -0.52 & 50.926 & 18 \\
\hline Scenario 4 & 1.000 & 1.000 & 1.000 & 1.000 & 0.953 & 0.991 & 10.920 & 0.932 & $2-0.33$ & 70.959 & 16 \\
\hline
\end{tabular}

In sum, PDF Map was restructured by disconnecting feedback loops and adjusting the signs of weights in order to solve the issues resulted from the existence of feedback loops and the wrong assignment of the weight. RPDF Map (the restructured PDF Map) has a simpler structure compared with the original PDF Map, which suppressed a limit cycle or "Not converged" responses in simulation. As a result, the simulation for Scenario 2 yielded converged responses. Therefore, in the CDRM process, it is necessary to revise mathematically aggregated FCM carefully and repeatedly from the viewpoint of engineering design.

\subsubsection{Sensitivity Analysis}

According to Hamby [282], a sensitivity analysis is conducted for a number of reasons in complex engineering phenomena. One of the reasons is to determine which 
inputs influence mostly on output variability. In this part, sensitivity analyses are performed to test how sensitively the result of each scenario changes along with changing its fundamental PDFs. In other words, the result of each analysis presents the change in each upper-level PDF affected by changing the activation value of a fundamental one included in the scenario.

Each fundamental PDFs in the scenario tests was regarded as an adjustable quantitative feature or function in engineering design. Particularly, the performance of "Advanced Navigation" (ANV) can be measured by the level of autonomous mobility [283]. For the sensitivity analyses, each fundamental PDF was activated between 0.1 and 1 with a step size of 0.3 . The FCM simulation applied the same condition of the scenario tests.

Figure 9.20 depicts the results of the sensitivity analyses for Scenario 1, prioritizing the battery performance of UniCleanBot.
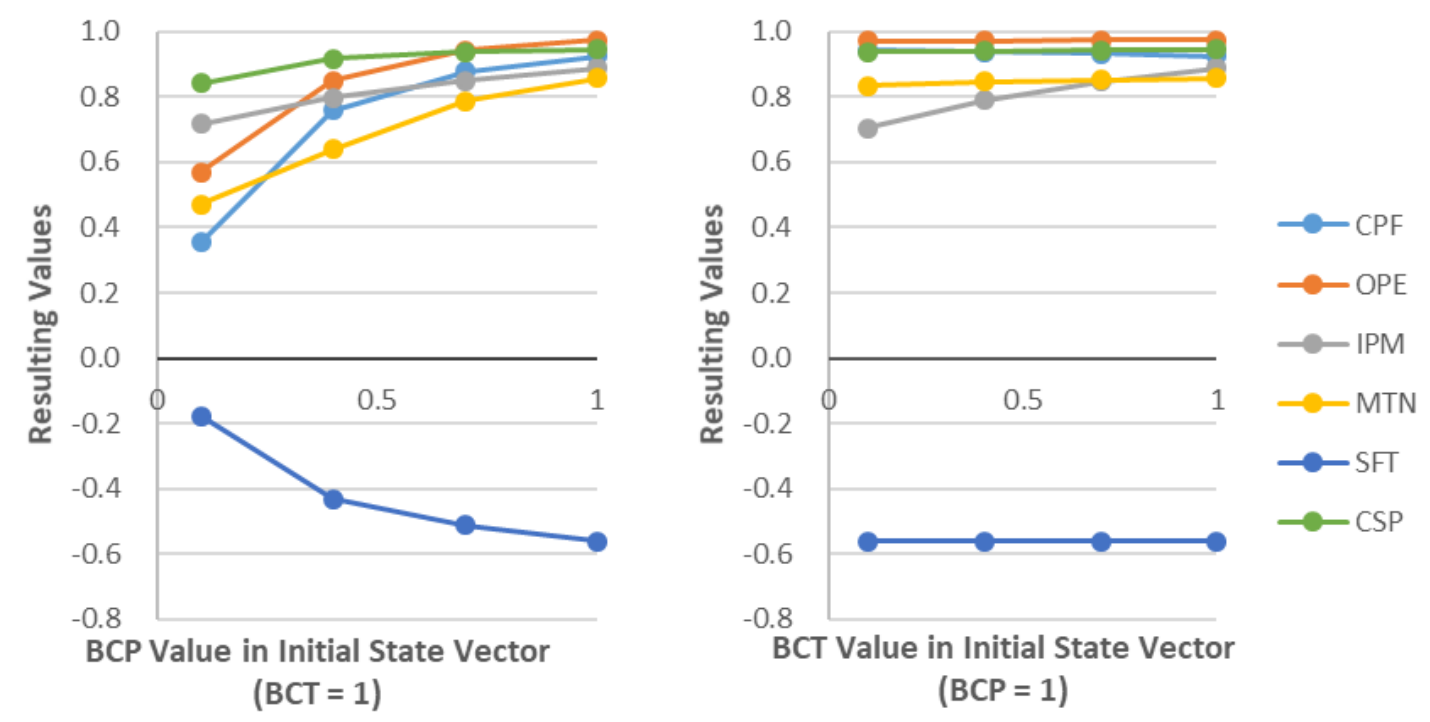

Figure 9.20 Sensitivity analysis for Scenario 1 
The left chart presents the changes in the upper-level PDFs' values correlated with the changes in the BCP value in the initial state vector with fixing the BCT value as 1 . Whereas the right one shows the resulting values of the upper-level PDFs along with the changes in the BCT value in the initial state vector with maintaining the $\mathrm{BCP}$ value as 1 .

When the $\mathrm{BCP}$ value changed with fixing the $\mathrm{BCT}$ value as $1, \mathrm{CPF}$ and $\mathrm{OPE}$ changed relatively larger than other upper-level PDFs. Particularly, the change of CPF is the biggest (0.569), while the CSP value changes minimally (0.103). Additionally, the SFT value was negative, unlike the others, and decreased when the BCP value increased. Consequently, the increase in the battery capacity of UniCleanBot leads to increasing customer preference while increasing customers' concern about its safety.

On the other hand, when the BCT value changed between 0.1 and 1 with fixing the BCP value as 1, most upper-level PDFs were not affected greatly ( less than 0.024) except for IPM (0.184). Moreover, when the BCT value increased from 0.1 to 1 , the CPF value decreased slightly (0.020). The SFT value was negative without any change by the change in the BCT value, which implies that, in this scenario, customers may concern the safety of UniCleanBot though the change in the battery charging time does not affect it.

From the sensitivity analyses for Scenario 1, prioritizing the battery performance may lead to customers' concern about the safety of UniCleanBot while customers may prefer it regardless of prioritizing the battery capacity and the battery charging time in engineering design. Therefore, in this scenario, engineering design needs to prepare alternatives to enhance the safety of UniCleanBot. 
Secondly, Figure 9.21 shows the results for Scenario 2, assuming that engineering design prioritizes operational convenience.

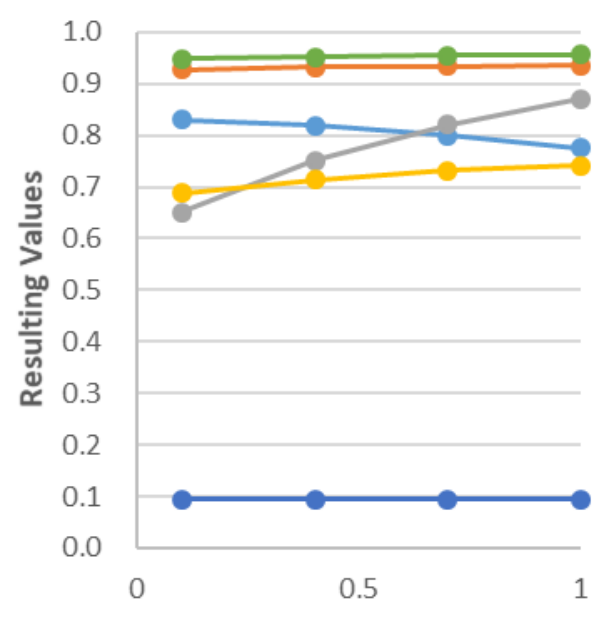

BCT Value in Initial State Vector $(\mathrm{ANV}=1)$

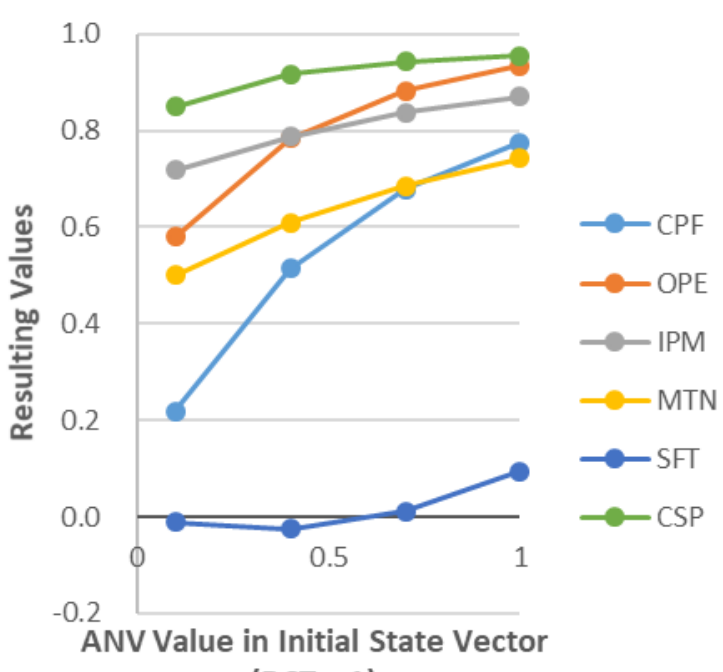

(BCT = 1)

Figure 9.21 Sensitivity analysis for Scenario 2

Similar to the results for Scenario 1, the increase in the BCT value with fixing the ANV value as 1 caused an increase in the IPM (0.221) and MTN (0.054), and a decrease (0.056) in CPF while relatively little changes (less than 0.008) in the others. However, the decrease level of the CPF value was larger than in Scenario 1. Interestingly, the SFT value was not affected by the BCT value change, but it has a positive value, unlike in Scenario 1.

By contrast, the change in the ANV value with fixing the BCT value as 1 led to relatively large changes in the upper-level PDFs compared to the previous case. Particularly, the CPF value increased most highly (0.556) by the increase in the ANV value. The OPE value also has a high increase $(0.355)$. The SFT value was negative when 
the ANV value was between 0.1 and 0.4 , but it changed to a positive value when the ANV value was 0.7 and larger.

Consequently, the results for Scenario 2 showed that the upper-level PDFs were sensitive to the ANV value change compared to change in the BCT value. All upper-level PDFs had positive values except for when the ANV value is under 0.7. Therefore, when engineering design has a high priority of "Advanced Navigation" in the concept design, customers may be favorable to UniCleanBot without any concerns.

Next, two sensitivity analyses were carried out for Scenario 3, assuming that engineering design prioritizes the cleaning coverage of UniCleanBot. The results are shown in Figure 9.22.
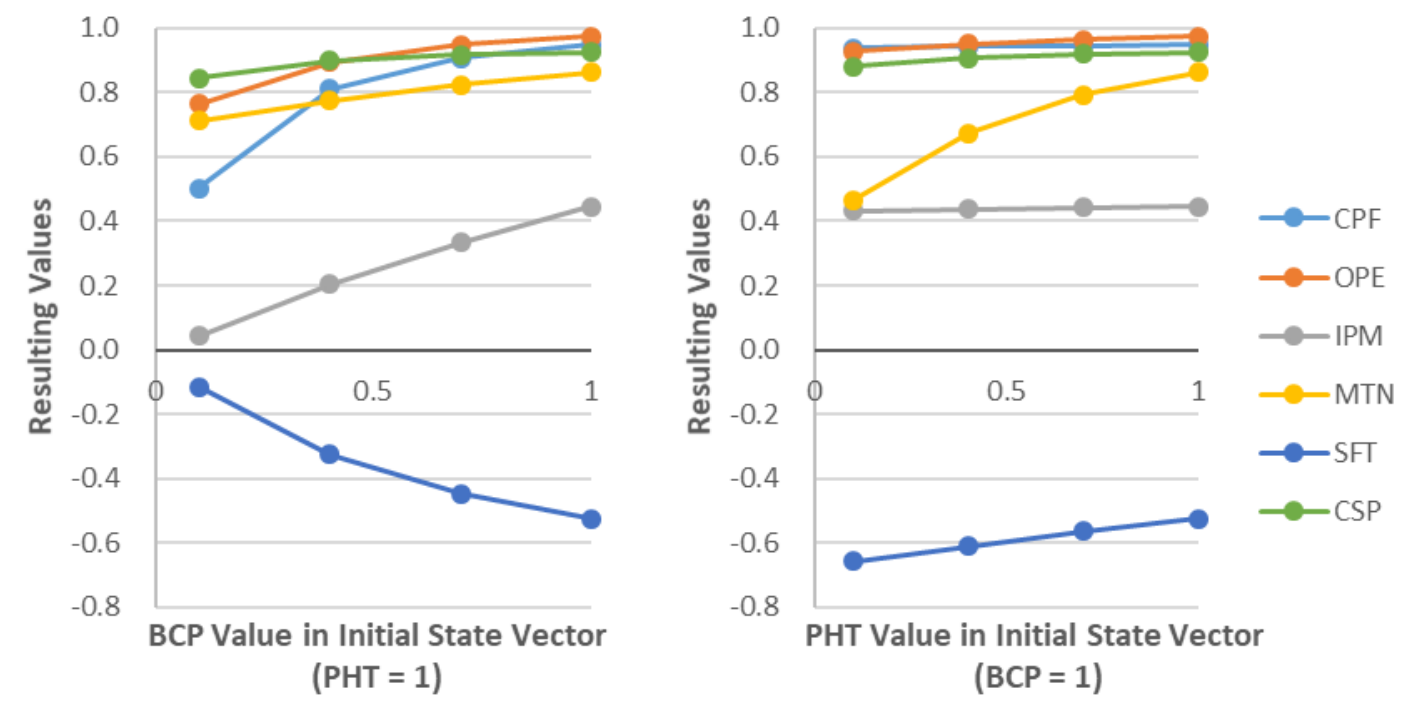

Figure 9.22 Sensitivity analysis for Scenario 3

When the BCP value changed from 0.1 to 1 without changing the PHT (fixing as 1), the SFT value decreased highly (410). Moreover, the increase in the BCP value led to high increases in the CPF (0.445) and IPM (0.400). However, the other three upper-level 
PDFs (OPE, MTN, and CSP) did not change greatly compared to the aforementioned three. On the other hand, the change of the PHT value impacts highly on the MTN $(0.396)$ and SFT (0.133) rather than the other upper-level PDFs.

In Scenario 3, prioritizing the cleaning coverage in the product concept may make customers favorable to UniCleanBot, but customers may concern about the safety.

Lastly, assuming that engineering design values the battery performance, the operational excellence, and the cleaning coverage as equals, four sensitivity analyses were conducted with changing values of four adjustable PDFs (BCP, BCT, ANV, and PHT). Figure 9.23 presents the results. 

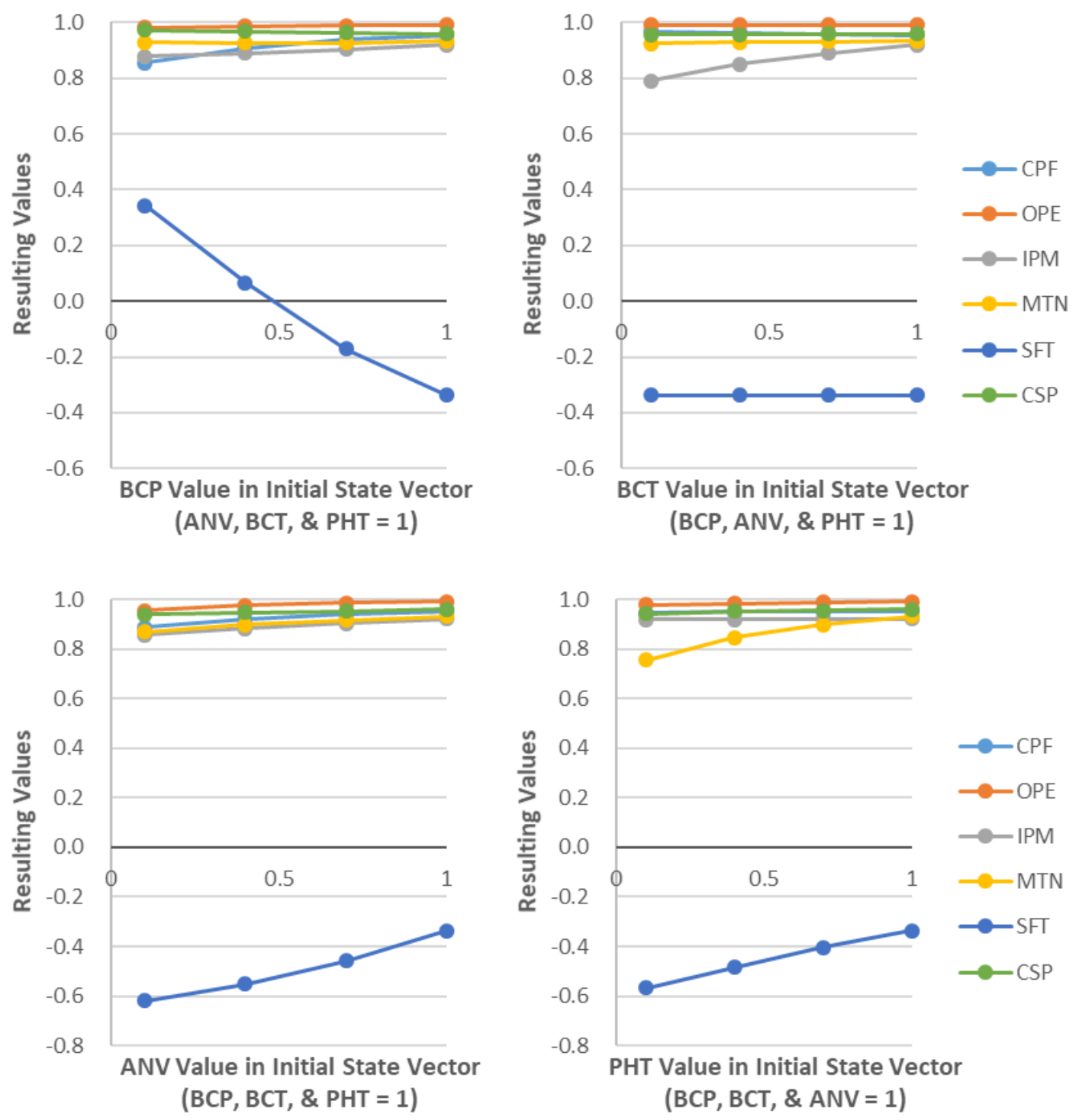

Figure 9.23 Sensitivity analyses for Scenario 4 
The upper left chart shows the results of a sensitivity analysis observing the change of the upper-level PDFs when the BCP value changes from 0.1 to 1 with fixing the other PDFs as 1. The change of BCP rarely affected the values of the upper-level PDFs besides SFT. The SFT value decreased greatly (from 0.342 to -0.337 ) by increasing the BCP value, which turned into a negative value when the $\mathrm{BCP}$ value was over 0.4 .

The upper right chart depicts the effect of the change in the BCT value on the upperlevel PDFs. Compared to the other upper-level PDFs, the IPM value changed relatively larger (0.231) when BCT increased. Furthermore, the SFT value stayed under 0 without any change.

The low left chart presents the impact of the ANV value change on the upper-level PDFs. The other values, BCP, ANV, and PHT were fixed as 1. The upper-level PDFs did not change greatly and had high values over 0.870 . The SFT value increased by the increase in the ANV, but it had negative values.

Lastly, the low right chart exhibits how the upper-level PDFs changed by the change in the PHT value with fixing the BCP, BCT, and ANV values as 1. The change in the PHT value led to distinctive changes in the MTN and SFT values while a few changes in the other upper-level PDFs. In addition, the SFT value was negative through the whole range of the PHT change.

Sensitivity analyses for the last scenario show that all upper-level PDFs retained high values besides the SFT values. Only the case of that the BCP value was less than 0.4 , the SFT value was positive. Therefore, if wanting to include all four product design 
factors in the product concept, engineering design needs to take care of an appropriate battery capacity avoiding to customers' concern.

In sum, it is confirmed that analyzing the sensitivity of the upper-level PDFs enables engineering design to determine which adjustable PDFs influence mostly on the upper-level ones. Furthermore, the sensitivity analysis of each scenario enables engineering design to avoid choosing a product design factor worsening customers' perception of a product benefit or customer preference.

\subsection{Survey Results}

\subsubsection{Background}

A total of three surveys were administered to trace changes in participants' understandings of how product design factor (PDF) impact customer benefits and, ultimately customer preference. Figure 9.24 shows a screenshot of a survey question. The full survey is available in Appendix C. 


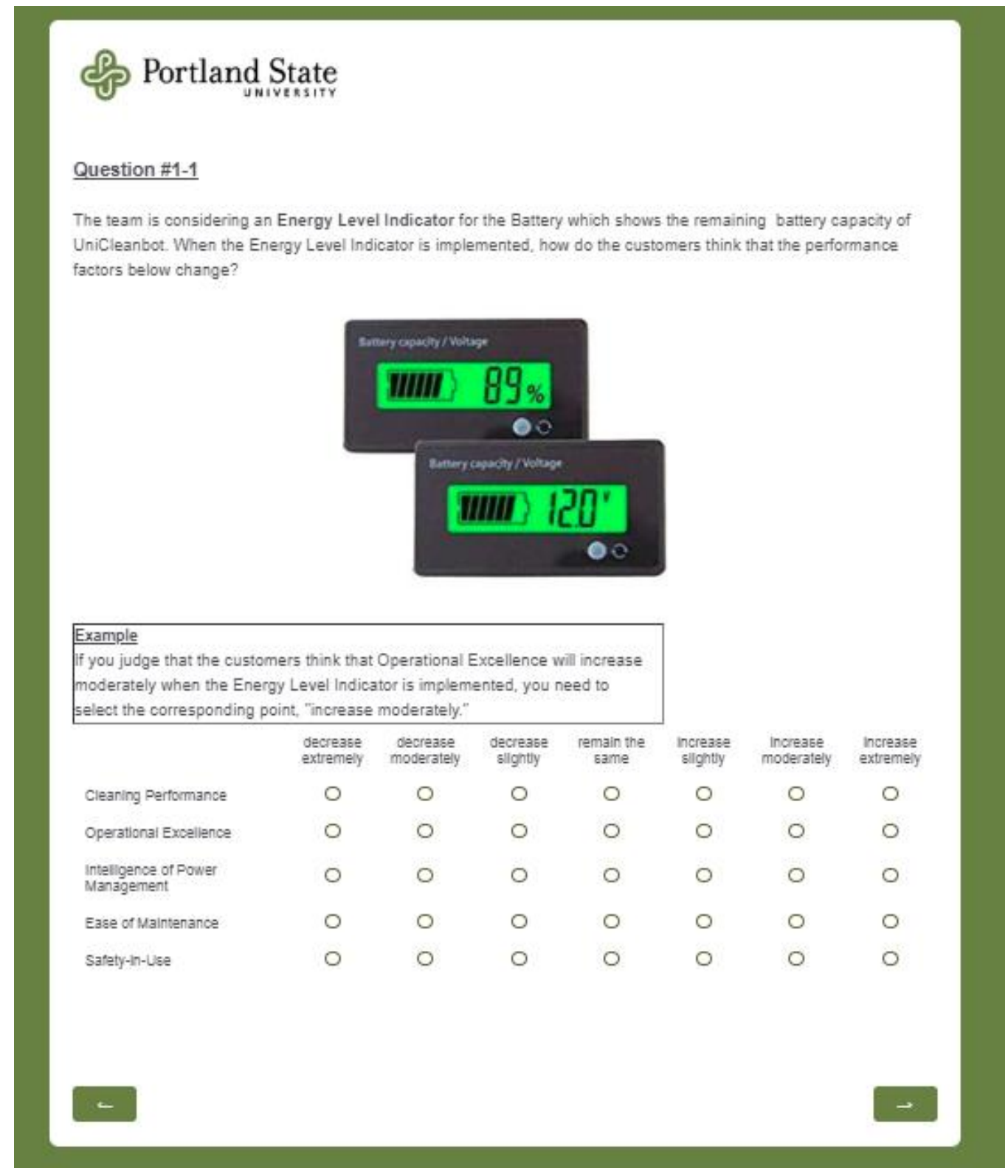

Figure 9.24 A screenshot of a survey questions

The core part of each survey remained the same to make it possible to compare survey results at different points in time. The core consisted of three types of questions:

The first set of questions asked, depicted in Figure 9.24 above, asked participants about changes to contributors to perceived customer benefits (namely, Cleaning Performance (CFP), Operational Excellence (OPE), Intelligent Power Management 
(IPM), Maintenance (MTN), and Safety (SFT)) in response to implementing one of eight product features: Energy Level Indicator (ELI), Water Proof Design (WPD), Face Recognition (FRE), Step-climbing Mechanism (SCM), Self-emptying Dustbin (SED), HEPA Filter (HEP), Expandable Corner Brush (ECB), and Profile Height (PHT). This question was intended to elicit tradeoffs. If, for example, the participant felt that a HEPA filter increases cleaning performance but leads to higher maintenance costs, he could have shown increases/decreases accordingly. Participants were asked to report their judgment on a seven-point Likert scale ranging from "decrease extremely" over "remain the same" to "increase extremely". For instance, if a participant judge that customers think that Operational Excellence (OPE) will increase moderately when the Energy Level Indicator is implemented, he/she needs to select the corresponding point, "increase moderately." The first type of questions thus provided insights into interdependencies between PDFs, which could be used for comparison with the FCM the participants provided.

The second type of question asked participants' judgment about customers' responses to the eight features described in the first question. ("Below is a list of Product Design Factors (or features) that the product development team could implement: In which way would implementing these features impact customer preference (i.e., how much customers like the product)?") The third type of questions asked for participants' estimation of how customers' preference level of the target product, UniCleanBot, would change if seven already existing product features (Battery Capacity (BCP), Suction Power (SPW), Water Tank Capacity (WTC), Battery Charging Time (BCT), Dustbin Capacity (DSP), Net Weight (NWT), and Product Height (PHT)) would be modified (Below are a list of already existing product features that the team could increase or decrease during 
product design. In which way would changing these features impact customer preference, i.e. how much customers like the product)?). The second and third types of questions thus provided insights into how participants think about the dynamic of the product concept, i.e., how a change in design changes outcomes, which could be compared against FCM dynamics.

Participants were asked to indicate their answers with a seven-point Likert scale. To analyze the result, every answer presented by a seven-point Likert scale is converted to a corresponding ordinal value as listed in Table 9.23

Table 9.23 Conversion of the Likert scale responses to ordinal values

\begin{tabular}{llc||llc} 
Question & Answer & $\begin{array}{c}\text { Ordinal } \\
\text { Value }\end{array}$ & Question & Answer & $\begin{array}{c}\text { Ordinal } \\
\text { Value }\end{array}$ \\
\hline Q1 & decrease extremely & 1 & Q2 \& Q3 & Much worse & 1 \\
& decrease moderately & 2 & Moderately worse & 2 \\
decrease slightly & 3 & Slightly worse & 3 \\
& remain the same & 4 & About the same & 4 \\
increase slightly & 5 & Slightly better & 5 \\
& increase moderately & 6 & Moderately better & 6 \\
& increase extremely & 7 & Much better & 7 \\
\hline
\end{tabular}

\subsubsection{Change across surveys for individual participants}

To compare how responses to the same questions differ across time (administered at three different times), responses can be mapped onto a chart. Figure 9.25 illustrates this approach and shows answers by the eight participants (E1-E8) to the 7th sub-question of Question 2 (asking a participant's judgment about change in customer preference of UniCleanBot by implementing extendable corner brush). This chart explains each participant's judgment on how customers perceive the impact of implementing 
Extendable Corner Brush (ECB) into a product concept on Customer Preference (CSP). In the chart, E3 responded that customers think that CSP will increase extremely when ECB is implemented into UniCleanBot in all surveys consistently. Whereas, the responses of E2, E4, E6, and E8 changed in each survey. The range of all responses for the three surveys is between four (About the same) and seven (Much better). Thus all participants evaluated that ECB affects CSP positively from customers' viewpoints by and large. Furthermore, the width is narrowed between Survey 2 and 3. This example shows that it is difficult to see a distinct pattern in the responses, simply by looking at the visualization because the participants do not all agree. Moreover, responses to other questions have a different shape. Appendix $\mathrm{H}$ contains the entire fifty-five charts of the survey data.

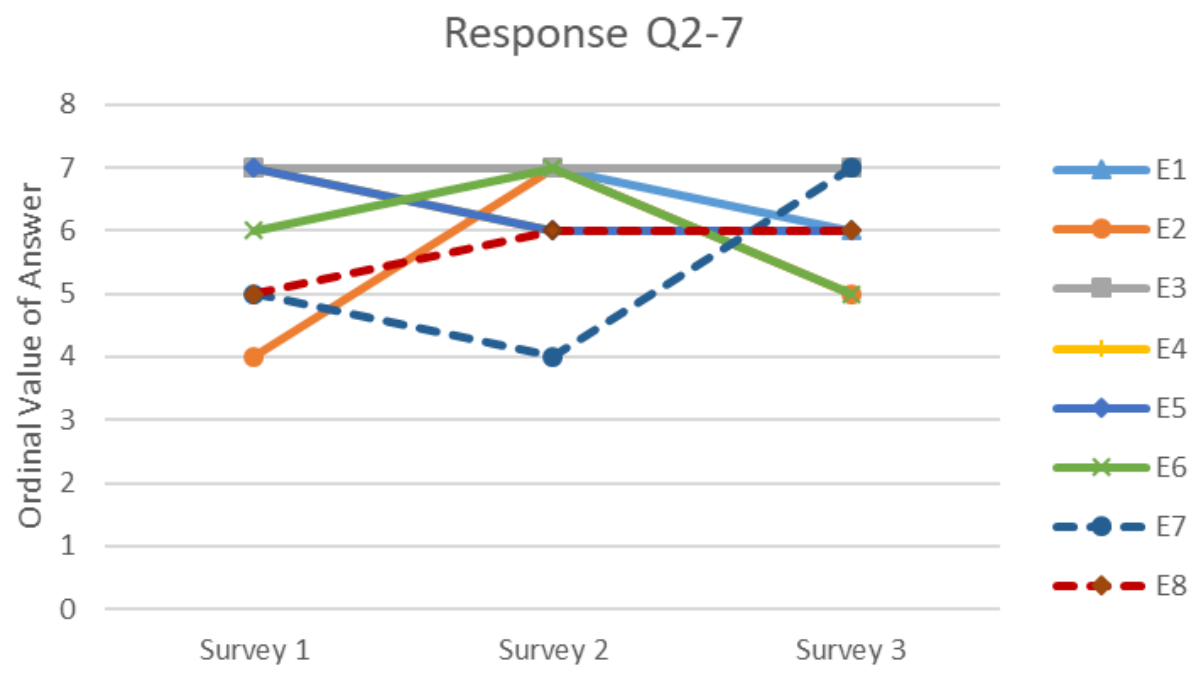

Figure 9.25 An example of response change a question during experiment

In response, statistical analysis was carried out for Likert scale surveys following the recommendations by Sullivan et al. [284] and Harpe [285]. Firstly, the central tendency and the dispersion of all eight responses to each question are measured with the 
mode, the median, the inter-quartile range (IQR), and the range. In order to see a change in the response of each question between the three surveys, three responses of a question in each survey are compared with each other for each analysis item. Table 9.24 shows a part of the comparison results as an example.

Table 9.24 A part of the comparison results of central tendency and dispersion in the three surveys

\begin{tabular}{|c|c|c|c|c|c|c|c|c|c|c|c|c|c|}
\hline \multirow{3}{*}{ 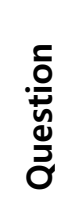 } & \multirow{3}{*}{ 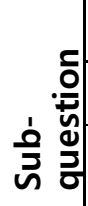 } & \multicolumn{6}{|c|}{ Central Tendency } & \multicolumn{6}{|c|}{ Spread/Dispersion } \\
\hline & & \multicolumn{3}{|c|}{ Mode } & \multicolumn{3}{|c|}{ Median } & \multicolumn{3}{|c|}{ IQR } & \multicolumn{3}{|c|}{ Range } \\
\hline & & Sv1 & Sv2 & Sv3 & Sv1 & Sv2 & Sv3 & Sv1 & Sv2 & Sv3 & Sv1 & Sv2 & Sv3 \\
\hline \multirow[t]{5}{*}{$1-1$} & 1 & 4 & 4 & 4 & 4 & 4 & 4 & 1 & 0 & 1 & 3 & 2 & 3 \\
\hline & 2 & 5 & 6 & 5 & 5 & 5 & 5 & 1 & 2 & 1 & 3 & 3 & 2 \\
\hline & 3 & 4 & 5 & 4 & 5 & 5 & 6 & 3 & 1 & 2 & 3 & 3 & 3 \\
\hline & 4 & 4 & 5 & 7 & 5 & 5 & 5 & 2 & 2 & 3 & 4 & 3 & 4 \\
\hline & 5 & 5 & 4 & 4 & 5 & 4 & 4 & 1 & 0 & 3 & 3 & 1 & 3 \\
\hline
\end{tabular}

In Table 9.24, all mode values of the three responses to the fourth sub-question are different from each other. It means that the majority of participants changed their answers to the sub-question through all three surveys. Also, the range values of the fourth subquestion are relatively bigger than the others, so that the dispersion of the responses are widely distributed rather than the others. While this analysis presents the overall status and the change of the central tendency and the dispersion for each response in the three surveys, it is difficult to see if and to what degree the participants' responses converge or diverge.

As mentioned in the previous chapter, a degree of consensus provides a good measure of response convergence for ordinal data such as Likert scale data. Initially, 
based on the response data of the three surveys, I calculated the consensus measure expressed in Equation (10) for participants' responses to each question. Next, it was observed how a consensus measure (a numerical expression for a degree of consensus) of an identical question changes through the three surveys. Subsequently, to trace the entire tendency of the consensus measure changes, the statistical distribution of the consensus measures for a question in each survey was depicted using a box and whisker plot graphically. In other words, a bar and whisker plot summarizes and shows the upper and lower quartiles (or interquartile range, named IRQ), medians, means, highest and lowest values of the consensus measures for each question in the three surveys.

Figure 9.26 presents the distributions of the consensus measures for the responses to Question 1 in the three surveys. Each measure of the distribution was calculated with the consensus measures for forty sub-questions under Question 1. From the top, each boxand-whisker plot corresponds to the consensus measure distribution of Question 1 in Survey 1, Survey 2, and Survey 3. The horizontal axis represents the consensus measure. The values above each plot represent the lowest value, mean, and highest value accordingly from left to right. Values below the plot stand for the lower quartile, median, and upper quartile. 


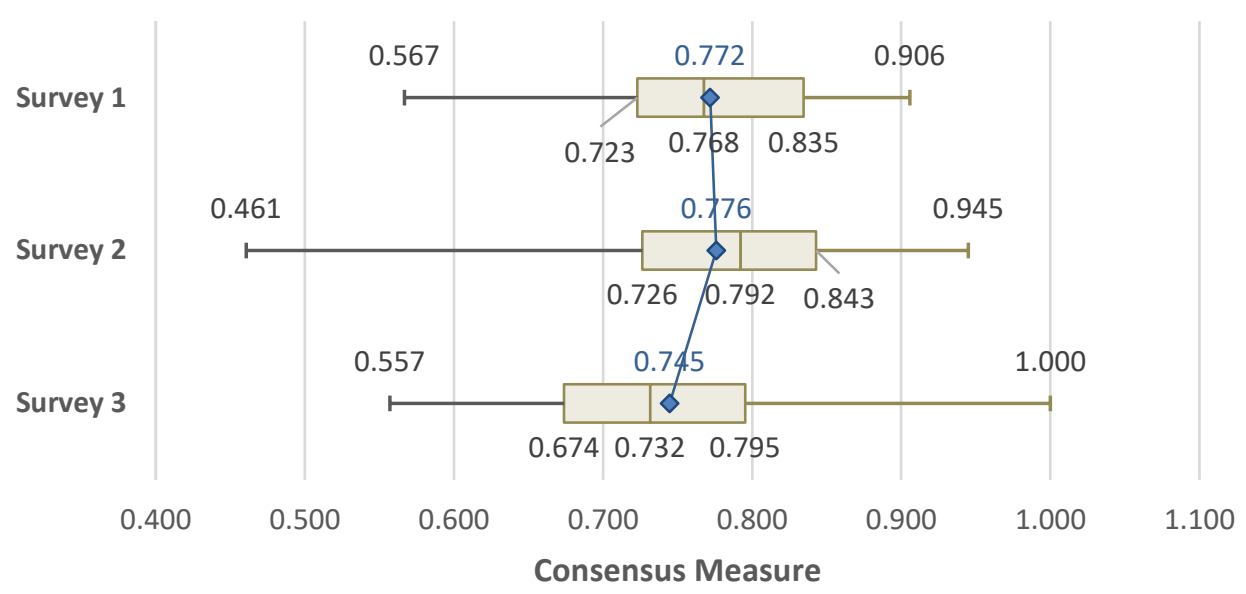

Figure 9.26 Distributions of Consensus Measures for Question 1

In Survey 1, the consensus measures are distributed from 0.567 to 0.906 , while the middle $50 \%$ of them are spread between 0.723 and 0.853 . The mean is 0.772 , and the median is 0.768 . The consensus measures range from 0.461 to 0.945 in Survey 2. The mean and median are 0.776 and 0.792 accordingly. Lastly, the consensus measures in Survey 3 are spread from 0.557 to 1.0 , and have 0.745 as the mean and 0.732 as the median.

This means that consensus about PDF interdependencies does not appear to be affected much by either individual cognitive mapping (between survey 1 and 2), nor the group discussion (between survey 2 and 3). Survey 1 required participants to strongly build on their own technical knowledge of engineering tradeoffs, which were not discussed in the materials presented before the first survey. Moreover, there was a considerable discussion during the workshop about how to present interdependencies between different PDF and to what extent such interdependencies even existed. It is, 
therefore, not entirely surprising that the range is broad and has not been reduced during the workshop.

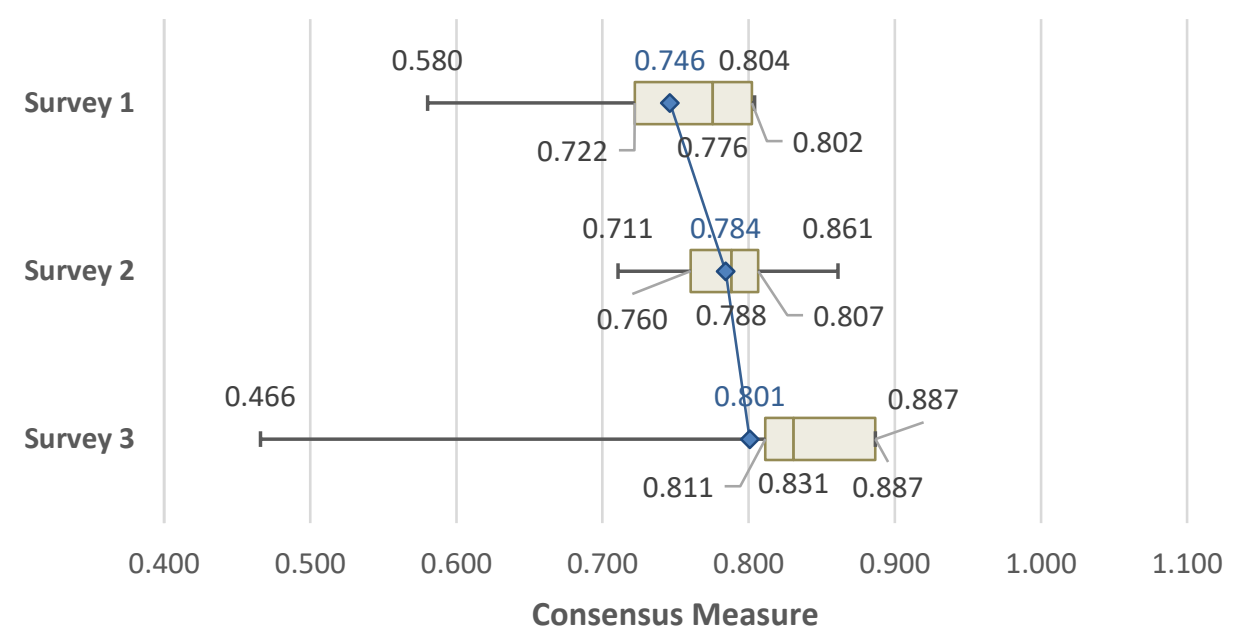

Figure 9.27 Distributions of consensus measures for Question 2

In comparison, participants could lean more heavily on the materials provided before the workshop in question 2 and 3, where they had to estimate the impact of PDF on customer preference. The consensus measures for the responses to Question 2 are depicted in Figure 9.27. Question 2 involves eight sub-questions asking the relationships between each of the interdependent product design factors (or trade-offs) and Customer Preference (CSP). The mean of each survey changes from 0.746 in Survey 1 over 0.784 in Survey 2 to 0.801 in Survey 3. The median is 0.776 in Survey 1, 0.788 in Survey 2, and 0.831 in Survey3. Regarding the range of the consensus measures, while the range in Survey 1 is from 0.580 to 0.802 , one in Survey 2 is between 0.711 and 0.861 . In Survey 3 , the range is placed between 0.466 and 0.887 . Interestingly, the plot shows that the difference between the lower quartile (0.801) and the median $(0.831)$ is relatively smaller than between the lowest value and the lower quartile so that the lower $50 \%$ of the consensus measures are distributed densely between the lower quartile and median. The 
lowest consensus measure in Survey 3 happened for the relationships between Face Recognition (FRE) and Customer Preference (CSP). Except for this measure, the others are spread out between the lower quartile and the highest value densely.

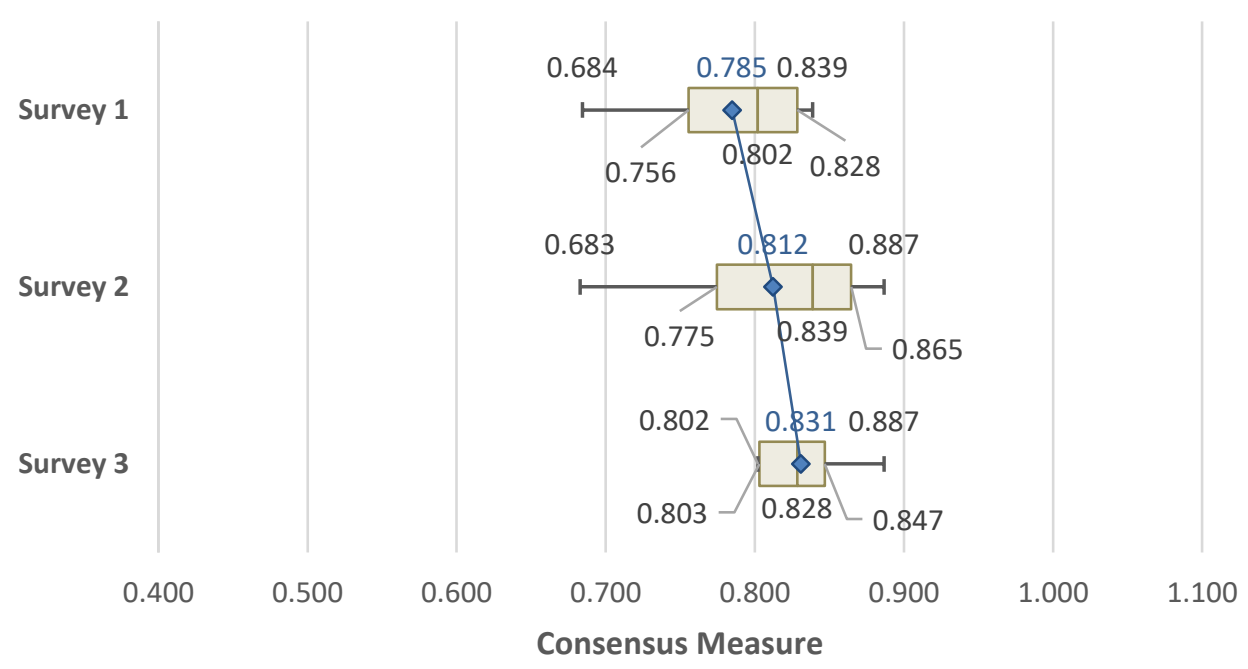

Figure 9.28 Distributions of consensus measures for Question 3

Lastly, Figure 9.28 shows the distributions of the consensus measures for the responses to Question 3 in the three surveys. Question 3 consists of the seven subquestions asking participants' estimation of how customers' preference level of UniCleanBot changes by increasing/decreasing each of seven existing product features. In Survey 1, the mean and the median are 0.785 and 0.802 , respectively. The distribution range of the consensus measures is between 0.683 and 0.887 , while the lower quartile is 0.775, and the upper one is 0.865 . The means in Survey 2 and Survey 3 are 0.812 and 0.831 , whereas the medians are 0.839 and 0.828 each. The range in Survey 2 is from 0.683 to 0.887 , and one in Survey 3 is from 0.082 to 0.887 . In particular, the dispersion of the consensus measures in Survey 3 is denser than the others. 
In sum, I had planned to observe how the participants' worldviews change during the experiment and assumed that there would be an increase in consensus and a narrowing of the range between the first and the second survey and the second and the third survey. For question 1, I could not observe such an effect, possibly because the participants were relatively uninformed about the engineering trade-offs involved in designing a home floor cleaning robot. For questions 2 and 3, the participants could lean on the materials that were provided before the workshop, including a product design requirements document and user personas. They were, therefore, more "on the same page" from the start because they had read the same materials, which might explain their higher level of consensus, which further increased during the group discussion. Accordingly, the results of the consensus measure calculations depicted in Figure 9.27, and Figure 9.28 show that the participants' responses to Question 2 and Question 3 converged when comparing the distributions of the consensus measures in Survey 1, Survey 2, and Survey 3. However, the effect was relatively small. Consequently, the analysis of the survey data cannot answer the question if the workshop activities, such as the individual and group FCM development during the workshop, enhance participants' mental models about the target product.

\subsubsection{Change in cognitive distance before and after modeling}

To confirm the effectiveness of CDRM, it is crucial to confirm whether the cognitive distances changed among the participants before and after the PDF Map development activity based on their individual FCMs. The participants' worldviews may change after exposing themselves to the PDF Map. Therefore, it is possible to confirm 
the change of participants' worldviews by comparing their FCMs collected before and after the PDF Map development activity.

Though the raw individual FCMs were collected once during the workshop, another set of individual FCMs should have been collected after the workshop for the comparison. However, because the PDF Map was not shared with the participants due to the limited time and technical issues, it was impossible to collect the other set of individual FCMs during the workshop. Furthermore, some participants had difficulty in having another meeting after the workshop, the researcher, therefore, decided to collect new individual FCMs from only two participants, namely those whose FCMs submitted during the workshop were distinctive from that of others. Comparison of structural, content, and behavior characteristics of individual FCMs analyzed in Subsection 9.1.2, highlighted the greate differences between two participants, E4 and E7. They were invited to separate 1:1 meetings with the researcher that took place after the workshop-

During each meeting, the researcher shared each participant's FCM, Tech Map and Need Map. Then, PDF Map and the simulation results were presented to each participant. Lastly, with the same software used for the individual FCM development activity during the workshop, each participant developed his/her new individual FCM.

Figure 9.29 presents a comparison between the raw and the new FCMs collected from the two participants, E4 and E7. The FCMs on the left side are the raw FCMs which the participants, E4 and E7, submitted during the workshop. The right side FCMs are the new FCMs that the participants developed after the workshop. Table 9.25 summarized 
the comparison of the structural characteristics and distance ratio between the raw and the new FCMs submitted by the participants, E4 and E7. 

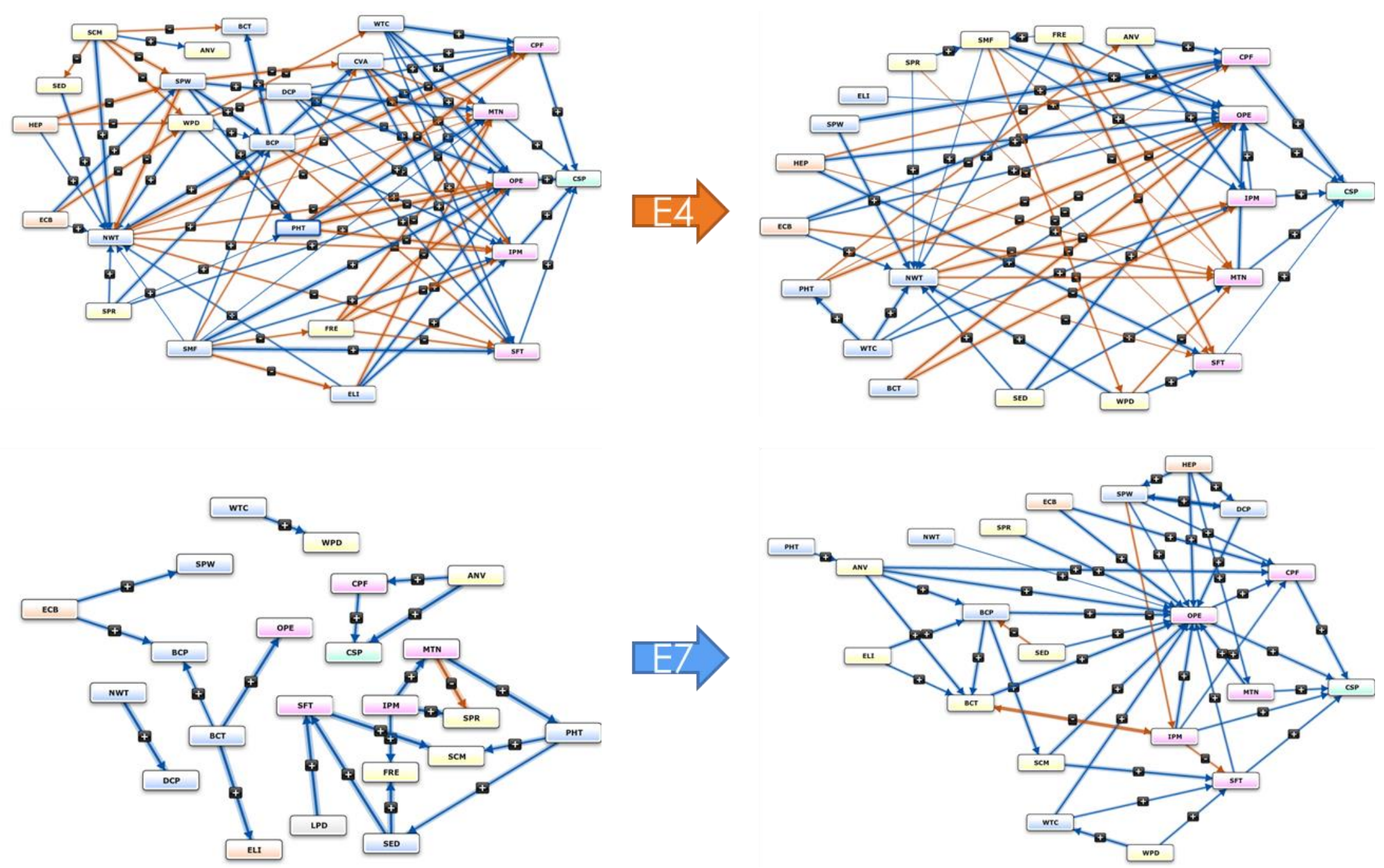
Table 9.25 Comparison of the structural characteristics and the distance ratios between the raw and the new FCMs

\begin{tabular}{|c|c|c|c|c|}
\hline \multirow[b]{2}{*}{ Comparison Criteria } & \multicolumn{2}{|c|}{ Raw FCMs } & \multicolumn{2}{|c|}{ New FCMs } \\
\hline & E4 & E7 & E4 & E7 \\
\hline Number of PDFs & 24 & 22 & 20 & 21 \\
\hline Number of Relationships & 80 & 21 & 52 & 46 \\
\hline C/N Value & 3.333 & 0.955 & 2.6 & 2.19 \\
\hline Density & 0.145 & 0.045 & 0.137 & 0.11 \\
\hline Number of Drivers & 5 & 7 & 9 & 8 \\
\hline Number of Receivers & 3 & 10 & 1 & 1 \\
\hline Number of Ordinaries & 16 & 5 & 10 & 12 \\
\hline Complexity Score & 0.6 & 1.429 & 0.111 & 0.125 \\
\hline Distance Ratio & \multicolumn{2}{|c|}{0.096} & \multicolumn{2}{|c|}{0.082} \\
\hline Number of PDFs in Distant Matrix & \multicolumn{2}{|c|}{25} & \multicolumn{2}{|c|}{23} \\
\hline Number of Common PDFs to Both Matrices & \multicolumn{2}{|c|}{21} & \multicolumn{2}{|c|}{18} \\
\hline Number of Unique PDFs in E7 & \multicolumn{2}{|c|}{1} & \multicolumn{2}{|c|}{3} \\
\hline Number of Unique PDFs in E4 & \multicolumn{2}{|c|}{3} & \multicolumn{2}{|c|}{2} \\
\hline
\end{tabular}

As a result, it is confirmed that the distance ratio between E4's and E7's FCMs was reduced through the experiment by exposing the two participants to Tech, Need, and PDF Maps.

To answer the fifth research question ${ }^{20}$ better, the research needs to provide evidence about whether the participants' understanding of product design factors has been improved through the CDRM process. As depicted in Figure 2.8, the improvement of the participants' understanding means, in this research, that the understanding of product development engineers approaches to the customers' worldview. Therefore, measuring

\footnotetext{
${ }^{20}$ Does CDRM result in an improved and shared understanding of product design factors among product team members?
} 
the change of the distance ratio between Need Map and each individual FCM at different times.

Figure 9.30 presents the distance ratio changes between Need Map at each FCM of participant E4 and E7 at different times (collected during and after the workshop). The distance ratio between Need Map and E4' FCM decreased with the value difference of 0.011 (from 0.065 to 0.051 ), while between Need Map, and E7's FCM increased with the value difference of 0.006 (from 0.045 to 0.051 ). Therefore, comparing the mean values of the distance ratios at the different times, the distance ratio between Need Map and the two individual FCMs decreased from 0.055 to 0.051 (the value difference of 0.004 ) though the degree of value is relatively small. Therefore, the understanding of product design factors among two participants has been approached slightly to the customers' worldview, so that the result explains that the participants' understanding of product design factors has been improved through the CDRM process. However, to make a firm conclusion, more individual FCMs should have been collected from the other six participants after the workshop.

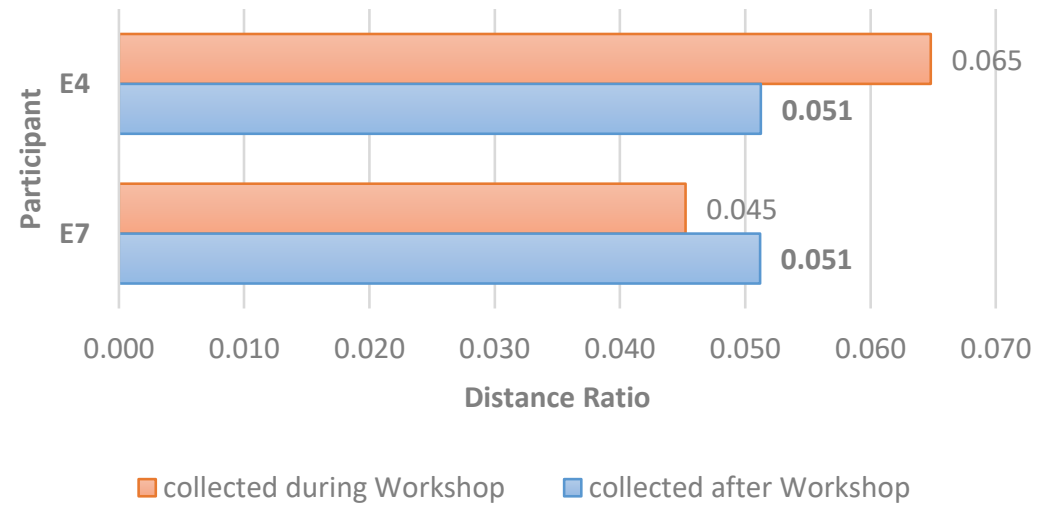

Figure 9.30 Distance ratio changes between Need Map and each individual FCM of participant E4 and E7 


\subsection{A Comparison between Simulation and Surveys}

This part describes the comparison between the survey responses and the simulation results with activating each of some PDFs, which are optional or adjustable in engineering design based on PDF Map.

During the experiment, the participants took three surveys asking their cognition on the impact of optional PDFs, like product features, in engineering design on the upperlevel ones in order of the experiment procedure. The first and second type questions asked how seven optional PDFs would affect the six upper-level ones (as benefits and customer preference) when they are applied to UniCleanBot. The seven optional PDFs are Energy Level Indicator (ELI), Water Proof Design (WPD), Face Recognition (FRE), Stepclimbing Mechanism (SCM), Self-emptying Dustbin (SED), HEPA Filter (HEP), and Expandable Corner Brush (ECB). The simulation for this analysis applied the same condition used in the scenario simulation in the previous section. The activation value of each PDF was set according to whether it is applied in engineering design. For instance, if ELI is included in UniCleanBot, the activation value is one while the other values are zero in the initial state vector.

Table 9.26 summarizes the resulting values of the upper-level PDFs affected by the activation of each optional PDF by simulation. All simulations did not result in the "Not Converged" state and were converged within the $35^{\text {th }}$ iteration. The followings are the comparison of these results with the survey responses: 
Table 9.26 Simulation results for the impact of the eight target PDFs

\begin{tabular}{llllrrrrr} 
& \multicolumn{2}{c}{$\begin{array}{l}\text { Activation } \\
\text { Value }\end{array}$} & \multicolumn{1}{l}{ CPF } & \multicolumn{1}{l}{ OPE } & \multicolumn{1}{l}{ IPM } & \multicolumn{1}{c}{ MTN } & \multicolumn{1}{l}{ SFT } & \multicolumn{2}{c}{ CSP } & \multicolumn{2}{c}{$\begin{array}{c}\text { Converged } \\
\text { Iteration }\end{array}$} \\
\hline ELI & 1.000 & 0.373 & 0.657 & 0.361 & 0.704 & -0.136 & $\mathbf{0 . 8 4 8}$ & 33 \\
WPD & 1.000 & 0.400 & 0.700 & 0.148 & -0.408 & 0.554 & $\mathbf{0 . 7 5 0}$ & 35 \\
FRE & 1.000 & 0.433 & 0.755 & 0.610 & 0.549 & 0.316 & $\mathbf{0 . 9 1 4}$ & 19 \\
SCM & 1.000 & -0.203 & -0.268 & -0.147 & -0.556 & -0.713 & $\mathbf{- 0 . 8 1 6}$ & 30 \\
SED & 1.000 & 0.772 & 0.846 & 0.096 & 0.164 & 0.672 & $\mathbf{0 . 9 0 8}$ & 21 \\
HEP & 1.000 & 0.078 & 0.599 & -0.080 & -0.450 & 0.390 & $\mathbf{0 . 4 3 8}$ & 34 \\
ECB & 1.000 & 0.570 & 0.693 & -0.049 & -0.046 & -0.034 & $\mathbf{0 . 6 9 9}$ & 25 \\
\hline
\end{tabular}

Figure 9.31 depicts the comparison of simulation results to the survey results for the influence of "Energy Level Indicator" (ELI) on product benefits (Question \#1-1). The three survey results are depicted as bars (Sv.1, Sv.2, and Sv.3) while the simulation ones as red diamond-dots (Sim.). The survey responses in the chart are the median values of the eight participants' responses. The left vertical axis provides guidance of numerical information for the simulation results of each product benefits. The right vertical axis indicates the level of the survey responses explained in Table 9.23. 


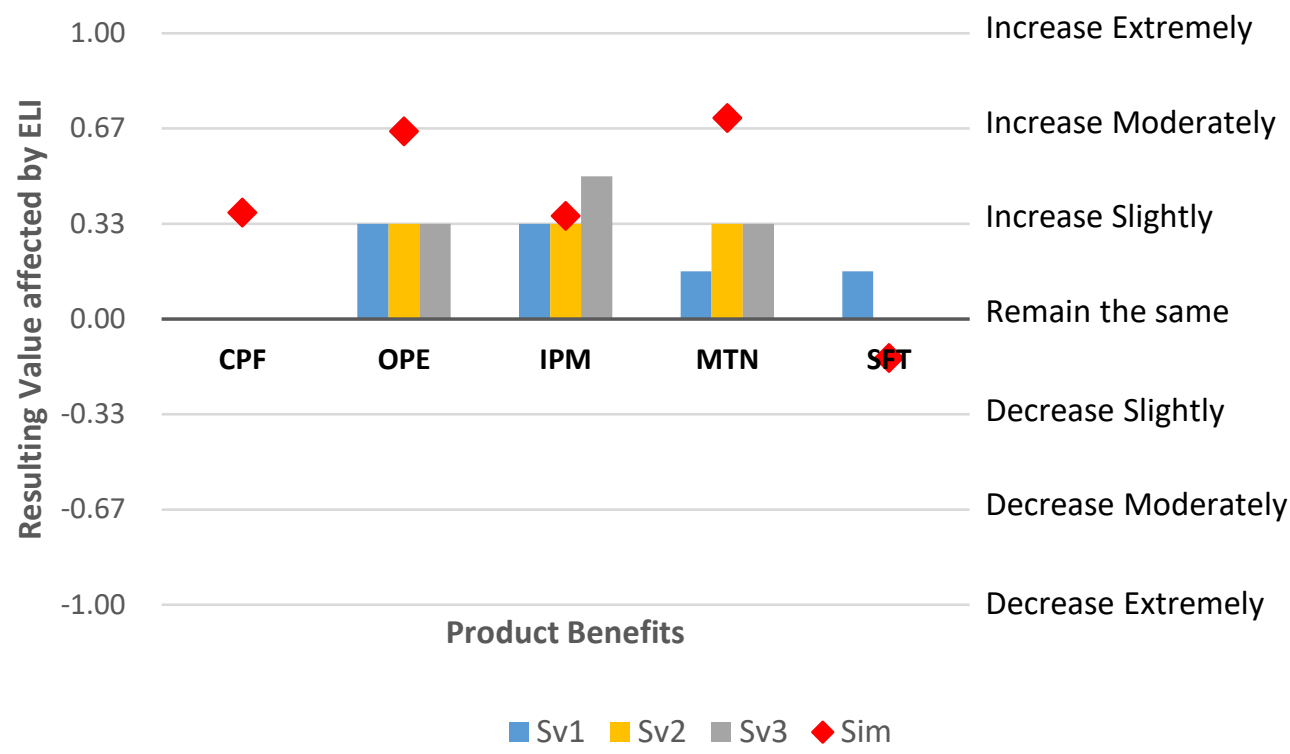

Figure 9.31 Comparison between simulation and surveys for Question \#1-1

ELI would lead to no change in CPF in the survey responses, while the activation of ELI caused relatively slight positive value (0.373) in the simulation. In the survey results, the participants answered that OPE and MTN would increase slightly when ELI was applied to UniCleanBot.

On the other hand, the activation of ELI output a moderate increase in OPE and MTN in the simulation. The activation of ELI caused a moderate increase in IPM in the simulation, whereas the participants responded that applying ELI would result in slightly and moderate increases in IPM. Lastly, SFT had a minute decrease in the simulation while the participants answered that it would have a slight increase or no change through the surveys.

Consequently, this comparison shows that the results of the simulation and the surveys about the effects of applying ELI present the same direction of change in CPF, OPE, IPM, and MTN though the difference in the level of strength. However, the results 
of the simulation and the surveys about the impact on SFT indicate different directions and levels of strength.

Figure 9.32 depicts the comparison of the simulation results to the three survey responses about the impact of the other six optional product design factors on the product benefits (Question \#1-2 to 7). The simulation results and the survey responses show similar tendencies of the directions and the levels of strength about the impact of SED and ECB on the product benefits in the middle- and bottom-right charts. However, unlike the two cases, the other four charts present different tendencies between the simulation results and the survey responses. Therefore, it is difficult to conclude that the simulation results are well-matched to the survey responses.

One possible interpretation from these results is that the three survey results the participants took at different times might fail to present the change in the worldview of the participant group by the FCM modeling activities during the workshop. (If the last survey responses approached to the simulation results rather than the others, we could conclude that the worldview of the participants had changed by the modeling activities.) Furthermore, the results imply another possibility that participants have difficulty in understanding the dynamic system intuitively between the features/functions and the benefits of a product. 

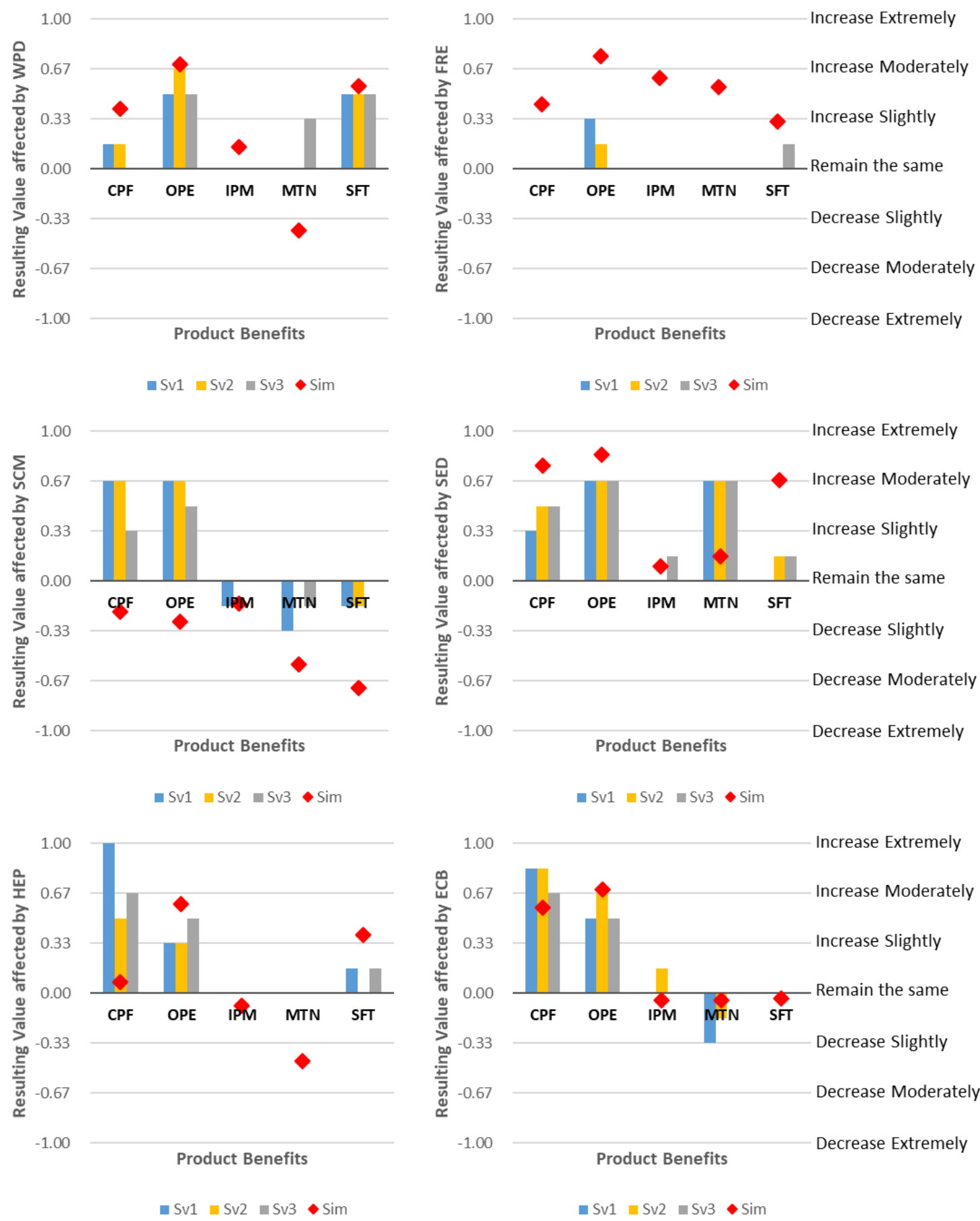

Figure 9.32 Comparison between simulation results and surveys for Question \#1-2 7

Next, Figure 9.33 depicts that the simulation results about the impact of each alternative PDF on customer preference compared with the survey responses (Question \#2). The simulation results show similar tendencies with the survey responses when 
activating five alternative PDFs, ELI, WPD, SED, HEP, and ECB. In contrast, the CSP value output by activating FRE in the simulation has a large difference in the preference level from the survey responses though both results have the same direction. Moreover, applying SCM resulted in the opposite direction between the simulation result and the survey responses. Consequently, this comparison to test the impact of the alternative PDFs on customer preference shows a much similar tendency, except for the cases of applying FRE or SCM to UniCleanBot.

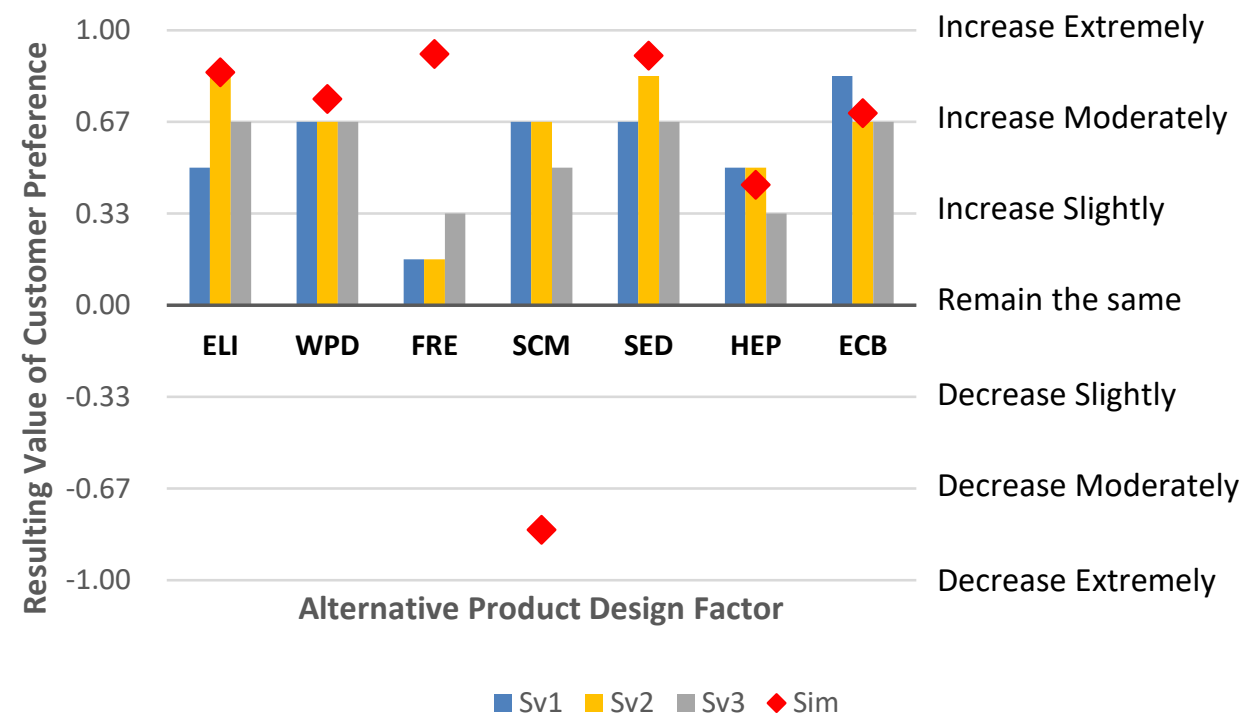

\section{Figure 9.33 Comparison between simulation and surveys for Question \#2}

Lastly, the third type question asked how customer preference would change by adjusting the priority level of seven alternative PDFs, as product features, that engineering design could increase or decrease for product concepts (Question \#3). The seven PDFs are Battery Capacity (BCP), Suction Power (SPW), Water Tank Capacity (WTC), Dustbin Capacity (DCP), Battery Charging Time (BCT), Net Weight (NWT), and Profile Height (PHT). For this comparison, the simulations applied the same 
condition used in the previous simulation to RPDF Map. To observe the quantitative change in CSP resulted from the level change in an alternative PDF, the range of each alternative PDF, as an activation element, was determined between 0.1 and 1 for the initial vector. This analysis drew the difference between the minimum and the maximum values of the resulting CSP when the activation value of each alternative PDF changed between 0.1 and 1 within the range that the simulation provides the converged result. Figure 9.34 presents the comparison results between the simulations and the survey responses for Question \#3.

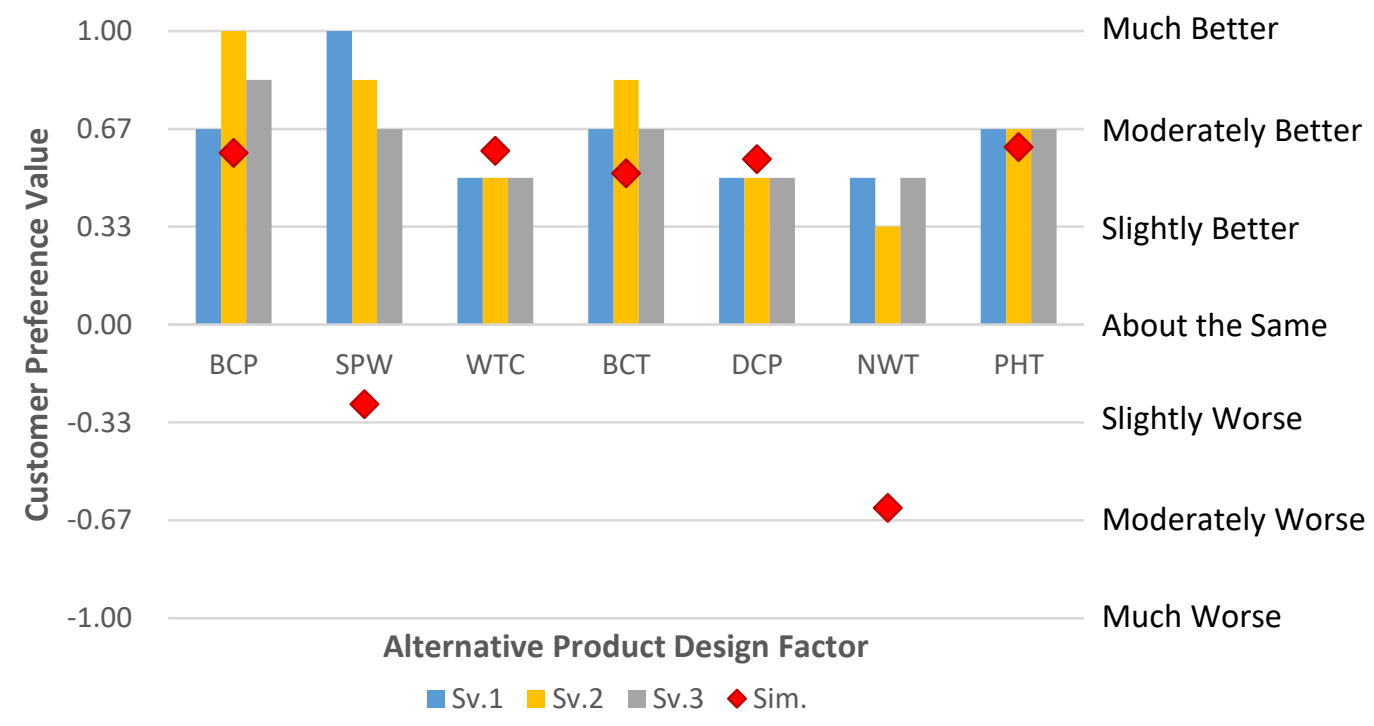

\section{Figure 9.34 Comparison between simulation and surveys for Question \#3}

Similar to the previous comparisons, the bars present the level of each survey response, and a red diamond-dot indicates the degree of the CSP value change caused by the change of each alternative PDF from 0.1 to 1 . Five (BCP, WTC, BCT, DCP, and PHT) of the seven alternative PDFs have the same direction and cognate levels of the changes between the simulation results and the survey responses. But, the simulation results 
yielded by activating the other two PDFs, SPW and NWT, demonstrate different directions of the change from the survey responses.

In conclusion, this part confirmed that the simulation results were partially matched to the survey results in the change directions of the upper-level PDFs affected by applying the alternative PDFs to product concepts in engineering design. The comparison for Question \#1 presented that the simulation results to test the impacts of applying optional PDFs on the product benefits were not matched well to the survey responses. It is supposed that the participants might have had difficulty in understanding the systemic complexity of the cause-effect relationships between the features/functions and the benefits of UniCleanBot, as aforementioned. On the contrary, the comparison results between the simulations and the surveys for Question \#2 and \#3 showed a similar tendency of the direction and the level of the CSP value change caused by applying each alternative PDF. Therefore, it is concluded that the participants have a better intuitive understanding of the causal links between the features/functions and the customer preference of UniCleanBot regardless of the systemic complexity in the product.

\subsection{Process Feedback}

Each of the second and the third surveys included questions asking the effectiveness of individual and group FCM development activities during the workshop. The first question in the second survey asked the participants to estimate the improvement of their understanding of the causal relationships between each PDF and customer preference of UniCleanBot as a result of the individual FCM development activity. Figure 9.35 presents the responses from the participants. Most participants answered that their understanding 
had been improved through the individual FCM activity in spite of the time limitation as mentioned in Subsection 7.3.6.

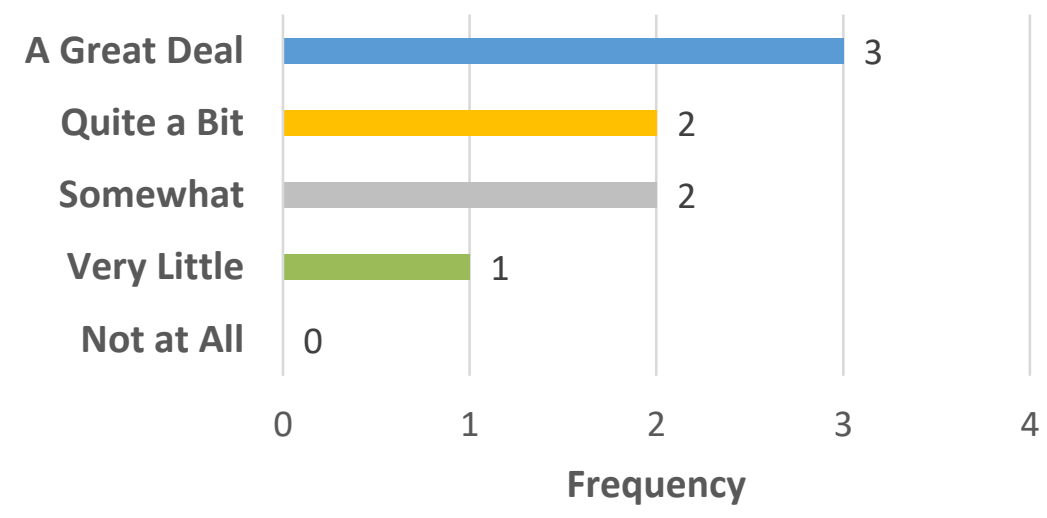

Figure 9.35 Participants' self-estimation of improvement of their understanding as a result of individual FCM development activity

Another question asked how helpful the individual FCM development was to understanding the relationships between each PDF and customer preference. Similarly, a majority of the participants answered that the individual FCM development was helpful for understanding the cause-effect relationships between each PDFs and customer preference as shown in Figure 9.36. 


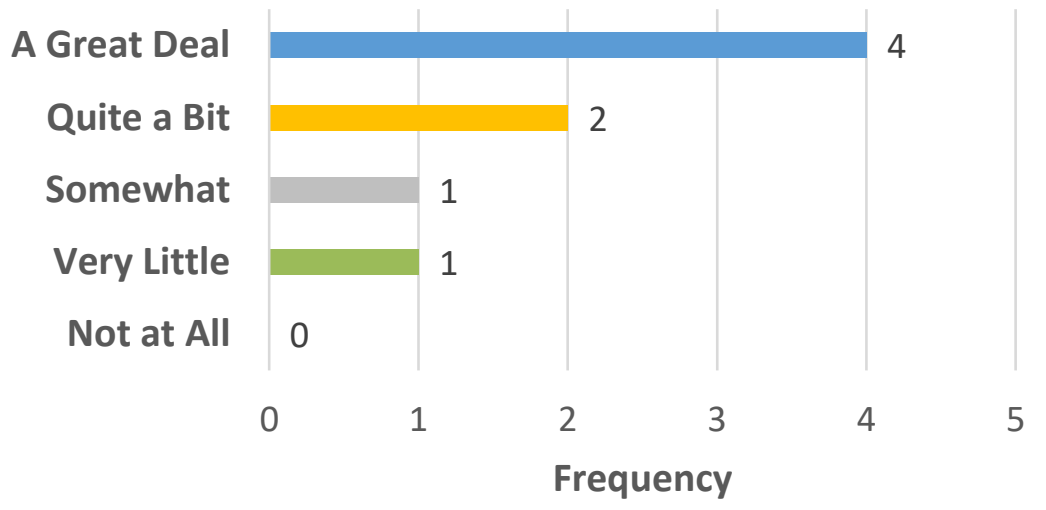

Figure 9.36 Participants' evaluation of the help of individual FCM development activity in enhancing their understanding

Lastly, through the third survey distributed after the workshop, the participants were asked how much improved was their understanding of causal relationships between each product design factor and the customer preference of UniCleanBot after the group FCM development activity during the workshop. As shown in Figure 9.37, all participants answered that their understanding was improved after the activity.

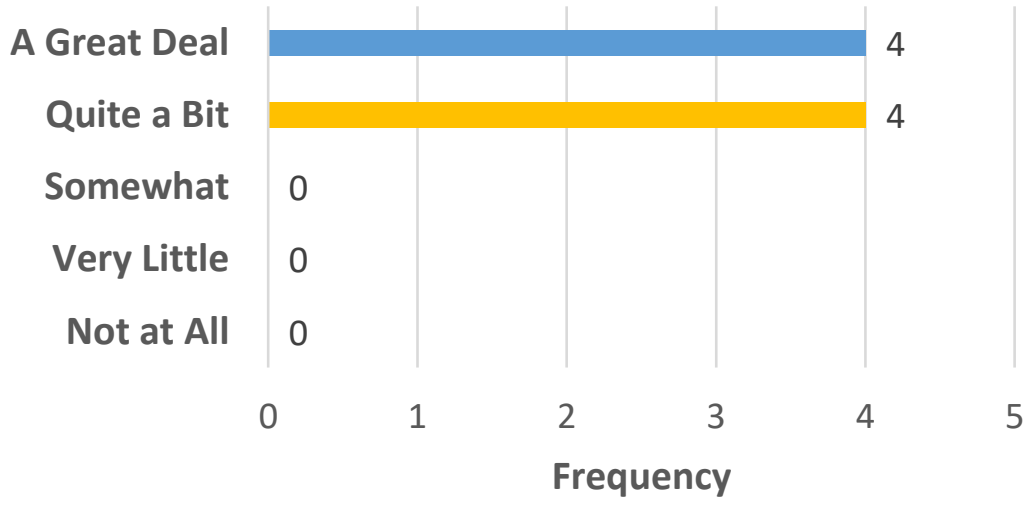

Figure 9.37 Participants' self-estimation of improvement of their understanding as a result of group FCM development activity

In sum, from the survey responses, the participants estimated their understanding had been improved by the modeling activities during the workshop. In addition, they 
evaluated the modeling activity was helpful in enhancing their understanding of the product system. This shows evidence of the effectiveness of CDRM with other results in the experiment described above. 


\section{DISCUSSION AND FUTURE RESEARCH}

This section discusses how the research results presented above contribute to answering the research questions posed in chapter 3. It also identifies areas for future research. As an overview, Table 10.1 summarizes the research questions and indicates how they were addressed through the feasibility pilot and the experiment. Each research question will be discussed separately.

Table 10.1 Chapters addressing corresponding answers to each research question in research implementation

\begin{tabular}{|c|c|c|c|c|}
\hline \multirow{3}{*}{ Research Question } & \multicolumn{4}{|c|}{ Answered by Research Implementation } \\
\hline & \multirow{2}{*}{$\begin{array}{l}\text { Feasibility } \\
\text { Pilot } \\
\text { Ch. } 6 \\
\end{array}$} & \multicolumn{3}{|c|}{ Experiment } \\
\hline & & Ch.7 & Ch.8 & Ch.9 \\
\hline $\begin{array}{l}\text { RQ. } 1 \text { How can the cognition of market- } \\
\text { oriented stakeholders and product } \\
\text { development engineers be modeled? }\end{array}$ & $\checkmark$ & $\checkmark$ & & $\checkmark$ \\
\hline $\begin{array}{c}\text { RQ. } 2 \text { Is it possible to integrate the separate } \\
\text { cognitive models of market-oriented } \\
\text { stakeholders and of PD engineers? }\end{array}$ & & $\checkmark$ & & $\checkmark$ \\
\hline $\begin{array}{l}\text { RQ. } 3 \text { How can alternative product concepts } \\
\text { be represented in the model as } \\
\text { alternative input scenarios? }\end{array}$ & $\checkmark$ & $\checkmark$ & & $\checkmark$ \\
\hline $\begin{array}{l}\text { RQ. } 4 \text { How can the outcomes of alternative } \\
\text { input scenarios be used to determine } \\
\text { the best product concept alternative? }\end{array}$ & $\checkmark$ & & & $\checkmark$ \\
\hline $\begin{array}{l}\text { RQ. } 5 \text { Does CDRM result in an improved and } \\
\text { shared understanding of product } \\
\text { design factors among product team } \\
\text { members? }\end{array}$ & & & $\checkmark$ & $\checkmark$ \\
\hline
\end{tabular}

\section{RQ 1.}

Research Question 1 asked how to represent the cognition of different stakeholders, namely of market-oriented stakeholders and product development engineers. In this work, I developed a modeling framework, consisting of different layers of PDFs and applied it 
in the Feasibility Pilot and in the Experiment. The specific approach differed in each study and for each stakeholder group. For market-oriented stakeholders, I moderated a group discussion with five consumers (in the Feasibility Pilot) or acted as the modeler, using an existing marketing requirements document as the basis for generating the model (in the Experiment). To represent the cognition of engineering stakeholders, I modeled my own and a colleague's expert knowledge as robotic engineers (Feasibility Pilot) and facilitated a modeling exercise during a workshop (Experiment), as described in Chapters 7 and 9.

In general, all approaches were able to represent stakeholder knowledge about interdependent aspects of the subject in the form of cognitive maps, leading me to conclude that the structure used by CDRM (different layers of PDFs) is comprehensible to participants. However, there appear to be considerable differences between participants with regard to ease and comfort with the modeling approach. Firstly, prior experience with the subject, not surprisingly, impacts the resulting models. The maps generated by more experienced engineers show higher overall complexity, indicating that they are more aware of interdependencies between PDFs. Secondly, modeling their own cognition does not necessarily come naturally to the participants. During the experiment, several participants struggled with the modeling format and some of their difficulties resulted in the need for additional refinement activities. These issues were much less prominent when the participants interacted with the modeler $1: 1$, as they did during the feasibility study.

These findings suggests that low interaction modeling with no or very little help from someone experienced in the method is not advisable. Therefore, for the practical use 
of CDRM, experienced modelers are necessary - they need to either "translate" participant input (e.g. from interviews) into an FCM model or create the model in collaboration with the participant during a cognitive mapping interview. Moreover, any integration of individual cognitive maps into a group cognitive map needs to be done by an experienced modeler before being presented to the group for discussion and refinement.

\section{RQ 2.}

Research Question 2 asked how the two different perspectives of 'market" and 'technology" can be integrated. In this study, I chose mathematical integration to combine individual FCMs into a Tech Map, and to integrate Tech and Need Maps to obtain a PDF Map. This was done by the modeler and not tested with the participants because CDRM assumes that participants are presented with the resulting model. Mathematical integration builds on prior works applied by Kosko [212] and Amer [286]. Because the CDRM structure was designed to have different layers of PDFs that each provide outputs for the next layer, it is very straightforward to do.

However, the resulting map was very big, complex, and likely too confusing for group discussions. Also, in the case of the experiment, the contributing models were done by some participants who had trouble with the modeling language, leading to inconsistencies. In response, I revised the model but it was nevertheless large and complex. I did not present this revised model to participants during the experiment.

From the lessons learned above, future research needs to find ways to make this complexity manageable in group discussions or for a modeler (e.g. through improved visualization or ways to interact with the model) for practical use of CDRM. 


\section{RQ 3.}

Research Question 3 asked how different product concepts can be represented as input vectors, which, in turn, permits the use of model simulation for assessing the consequences of selecting these concepts. To develop alternative product concepts based on the group FCMs (Tech and Need Map for the feasibility pilot, and PDF Map for the experiment), a minimum of three a critical product design factors (i.e., product features or functions) were identified in group discussions. Alternative product concepts were developed by creating several possible combinations of the selected factors. The alternative product concepts were used as scenario inputs for FCM simulations in the next step of CDRM.

\section{RQ 4.}

Research Question 4 fundamentally asked if CDRM simulation results can differentiate between product concepts with varying degrees of attractiveness and if simulation results can be used to select the best product concepts among given alternatives. To this end, I simulated the state of all concepts of the PDF Map, in response to the different input scenarios (product concept) discussed under research question 3. The simulation results allow a comparison of different product concepts in order to select the one that leads to the highest customer preference. CDRM can thus be used for concept selection. However, there are several aspects to consider:

First, FCM simulation results are meaningless by themselves because concept activation levels do not immediately translate into a real-world metric. If, for example, two product concepts lead to different simulation results for "ease of maintenance" (e.g. 
0.3 and 0.5 ), and as result to different levels of customer preference, we can determine that one concept is easier to maintain than the other and therefore preferred over the other. This supports product concept selection decisions. However, we cannot estimate the maintenance needs in hours, dollars spent, uptime, or any other engineering metric. We also do not know if the obtained level is good enough from a customer perspective - the relatively better product concept may still not perform well enough.

Second, if we choose the best product concept from a finite set of tested alternatives, it may not be an "ideal" product - a different combination of inputs could either lead to higher preference or that the same level of preference could be achieved with lower levels of input. In one case, opportunities for customer value are missed, in the other case, there might be overengineering.

This is why, thirdly, CDRM is intended to be an iterative process: simulation results are used to revise product concepts and test these new concepts by running the simulation again, this time with a revised input vector. New results are compared against prior ideas, additional concept ideas are generated and tested, etc. Thus product concepts are improved iteratively, rather than simply selected.

The concept development aspect of CDRM was not fully tested as part of this study and future research needs to investigate how to leverage CDRM in this use case and how it impacts the cognition and creativity of the PD team. However, I did investigate one potentially useful tool for product concept development, namely sensitivity analysis described in Section 9.2. In this analysis, I tested how sensitive a desired outcome (here: customer preference) responds to input changes by choosing input vector that not only 
represent binary states of PD factors (i,.e. PD is present $=1$ and not present $=0$ ) but varying degrees of inputs. This can be useful for product concept selection by identifying PDFs that, after meeting a threshold, do not contribute to further improvements of customer preference or might even have detrimental effects. Product concepts can thus be modified to meet necessary thresholds but not exceed them. In most cases, I could not identify a product concept that maximizes customer preference without also maximizing engineering inputs. Possibly, this is a result of the cognition of the participants (more is better mindset). However, in one instance, the sensitivity analysis shows a different result increase in battery capacity worsens customers' concern about safety, causing a decrease in customer preference in the fourth scenario. Therefore, sensitivity analysis enables engineering design to avoid the aforementioned issues (overengineering or not meeting minimum requirements). This interpretation of the sensitivity analysis results makes CDRM an overall promising means to not only assess product concepts but also develop new ones.

However, more work needs to be done. To make sensitivity analysis easy to do and interpret, we likely need a standardized template and better tools for visualization. It also comes at a cost. In the presented study, participants only had to decide between binary states of product features (feature is present or not), which is cognitively simple. In these cases, it will be critical to clarify the meaning of the activation value for a quantitative product feature for practical applications of CDRM. (One possible option, when CDRM is applied in product development for an existing market, is to determine a range of physical properties a product feature of competing products has, then map the range onto between 0 and 1 which an activation value has in FCM simulation.) 


\section{RQ 5.}

Research Question 5 asked if CDRM results in an improved and shared understanding of product design factors among product team members. To observe the effectiveness of CDRM, I applied two measures, "degree of consensus" and "distance ratio," based on surveys and a modeling workshop. However, as opposed to a real-world application of CDRM, in which the PD team would engage with the model multiple times and possibly over several weeks, while product concepts are refined, participants in this study had much more limited opportunities for learning during a single workshop. Moreover, as opposed to a PD team in a company, they came in with limited subject matter knowledge and experience. Consequently, the assessment with regard to RQ 5 is incomplete.

Nevertheless, the data from the experiment show some evidence that participants learned about the system (consisting of product design factors and relationships among them) and aligned their cognitive model more with those of other participants:

First, the participants self-reported a relatively large effect of each FCM development activity for their understanding of the system. Also, the consensus measures among the participants indicated increased agreement with every survey, however, the change is small on average. Moreover I investigated the distance ratio before and after the group modeling exercise by focusing on the cognitive maps of two "extreme" participants who, with regard to the characteristics of their FCMs differed strongly from each other and from other participants. I found an increasing alignment between them. 
Combined, this hints at the possibility that CDRM resulted in an increased agreement between the participants. However there is a discrepancy between the relatively high levels of self-reported learning and the limited learning (in the sense of increased alignment with the group) that was actually observed. This has several possible explanations: It might be the result of the phenomenon that people frequently value the instruction in a new method or way of thinking but that this instruction has a limited impact on actual decision making. It is also possible that some participants, who were not experienced in developing the target product, were simply inconsistent in their assessments or that some participants were extremely easily swayed by anything that was said, even if it did represent a widely agreed upon opinion. As discussed above, the experiment was very limited in scope. In future studies, I intend to repeat a similar experimental study with a real-world development team and the team's actual product to assess CDRM effectiveness.

In general, future research should occur in real-world settings and should track the impact of CDRM on learning longitudinally. 


\section{CONCLUSION}

\subsection{Research Summary}

All technology firms are concerned with product success because products that disappoint customers, do not sell well, or have insufficient margins are not only wasteful but harm competitive positions. As the basis of this work, I have undertaken an extensive literature review to understand the root causes of such failures. They are: inadequate understanding of markets and customers, misinterpretation of customer information and flawed product requirements, and, as a result, poor product concepts [27]. Though market research is important, these issues cannot be resolved solely focusing on better market information but also require that product development teams overcome the cognitive distance between their different engineering disciplines and market-oriented stakeholders to fully understand the interdependencies between product design factors (PDFs) [151][154], [157]. Currently, existing methods for product concept development are limited in their ability to do so.

To resolve the resulting challenges, this dissertation research developed a novel approach, a cognitive distance reduction method (CDRM), to enhance product development engineers' understanding of customer preference in product concept development. CDRM consists of six steps (Basic PDF Elicitation, Model Formation, Model Synthesis, Scenario Building, Simulation, and Result Analysis \& Interpretation). CDRM is based on a system modeling approach, namely fuzzy cognitive mapping (FCM), that is gaining popularity in many fields but is still largely unused in product innovation. To test and assess the proposed CDRM, I used two different approaches: a feasibility pilot and an experimental workshop. I collected and analyzed FCM models from both 
studies, as well as survey questionnaires. To analyze this data, I adopted several techniques not commonly used in FCM or new product development research and developed the necessary analytical tools in R and Python. In particular, I tracked changes in the workshop participants' understanding by calculating the degree of consensus based on the survey responses. I also developed approaches for comparing the distance ratios between the individual FCM submitted during and after the workshop.

As discussed in chapter 10, this research demonstrates that CDRM is capable of representing a new product as a system comprised of product design factors and relationships among them. Complexity is managed by creating the customer-focused Need Map and the engineering-focused Tech Map independently and integrating them to construct a group mental model, so-called PDF Map. The various maps capture the worldviews of PD team members and serve as a communication tool. Moreover, CDRM can also be used as a simulation tool and helps teams identify and select product concepts that achieve high customer value, given existing constraints. As part of the CDRM analysis and simulation, sensitivity analysis helps product development avoid overengineering or not meeting minimum requirements by identifying PDFs that do not contribute to further improvements of customer preference or might even have detrimental effects.

\subsection{Contributions}

The primary contribution of this research is of practical nature: it resulted in a novel approach that allows product development engineers to capture and understand customer knowledge for successful concept development activities in technology-driven firms, 
particularly those that emphasize user-centered innovation and co-creation with customers. CDRM is expected to improve current concept development practice by improving engineers' understanding of customer requirements and select product concepts that best fulfill customer needs.

To make these practical contributions possible, I furthermore proposed a new conceptual model to build team mental models and to improve knowledge sharing in new product development by applying the theoretical concept of cognitive distance. In line with the concept, this research also suggested a way of using a measurement tool, Distance Ratio, to assess the relative levels of cognitive distance among a group of people based on the contents of their mental models. In addition, Degree of Consensus, as a measure to estimate the level of consensus among a group, allows researchers to test the effectiveness of a group learning or sharing. Including these two measurement method, I developed new codes based on open-source packages using R and Python, which enabled me to carry out all calculations and visualizations for CDRM in this study. These methodological contributions can be used by practitioners, who wish to understand if their team aligns around the same project interpretation or is suffering from equivocality. In practice, this may be particularly useful for understanding and overcoming the root causes of task conflict.

The methods developed in this research will furthermore help other researchers. They are generally suitable for research that aims to understand how different people think about a complex system and to what extent their mental models align. Accordingly, there are many application domains. Specifically, in product development research, this 
work provides the foundations for understanding the needs and engineering tradeoffs in designing robotic vacuum cleaners. Future research may be able to leverage this to shed light on the relatively low adoption of this technology, despite its decades-long availability in the market place.

Theoretical contributions were not at the core of this work, yet it did provide a comprehensive discussion of several phenomena that plague early product development and knowledge sharing. Particularly, I propose a clear differentiation between uncertainty, complexity, and equivocality, describe how they impact team mental models and explain cognitive distance. I thus was able to integrate several current research perspectives and lay the theoretical foundation for the design of CDRM. 


\section{Bibliography}

[1] Q. Zhang and W. J. Doll, "The fuzzy front end and success of new product development: a causal model," Eur. J. Innov. Manag., vol. 4, no. 2, pp. 95-112, Jun. 2001.

[2] G. E. Okudan and S. Tauhid, "Concept selection methods - a literature review from 1980 to 2008," Int. J. Des. Eng., vol. 1, no. 3, pp. 243-277, 2008.

[3] K. T. Ulrich and S. D. Eppinger, Product Design and Development, 5th ed. New York, NY: McGraw-Hill, 2012.

[4] M. G. Luchs, K. S. Swan, and M. E. H. Creusen, "Perspective: a review of marketing research on product design with directions for future research," J. Prod. Innov. Manag., p. n/a-n/a, 2015.

[5] X. P. Zhang, J. Bode, and S. J. Ren, "Neural networks in Quality Function Deployment," Comput. Ind. Eng., vol. 31, no. 3-4, pp. 669-673, 1996.

[6] L. Y. Zhai, L. P. Khoo, and Z. W. Zhong, "Design concept evaluation in product development using rough sets and grey relation analysis," Expert Syst. Appl., vol. 36, no. 3 PART 2, pp. 7072-7079, 2009.

[7] D. Ullman, The Mechanical Design Process, 4th ed. New York, NY: McGraw-Hill Education, 2009.

[8] J. Sandström and J. Toivanen, "The problem of managing product development engineers: Can the balanced scorecard be an answer?," Int. J. Prod. Econ., vol. 78, no. 1, pp. 79-90, Jul. 2002.

[9] E. Fredericks, "Cross-functional involvement in new product development," Qual. Mark. Res. An Int. J., vol. 8, no. 3, pp. 327-341, Sep. 2005.

[10] T.-M. Yeh, F.-Y. Pai, and C.-C. Yang, "Performance improvement in new product development with effective tools and techniques adoption for high-tech industries,” Qual. Quant., vol. 44, no. 1, pp. 131-152, Jan. 2010.

[11] R. Ramaswamy and K. Ulrich, "Augmenting the house of quality with engineering models," Res. Eng. Des., vol. 5, no. 2, pp. 70-79, Jun. 1993.

[12] K. Kang, C. Kang, and Y. S. Hong, "Data-driven optimized vehicle-level engineering specifications," Ind. Manag. Data Syst., vol. 114, no. 3, pp. 338-364, 2014.

[13] V. Bouchereau and H. Rowlands, "Methods and techniques to help quality function deployment (QFD)," Benchmarking An Int. J., vol. 7, no. 1, pp. 8-20, 2000.

[14] L. Luo, P. . Kannan, and B. T. Ratchford, "Incorporating Subjective Characteristics in Product Design and Evaluations," J. Mark. Res., vol. 45, no. 2, pp. 182-194, Apr. 2008.

[15] J. J. Michalek, F. M. Feinberg, and P. Y. Papalambros, "Linking marketing and engineering product design decisions via Analytical Target Cascading," J. Prod. Innov. Manag., vol. 22, no. 1, pp. 42-62, Jan. 2005.

[16] P. G. Patterson, "Expectations and product performance as determinants of satisfaction for a high-involvement purchase," Psychol. Mark., vol. 10, no. 5, pp. 449-465, 1993. 
[17] A. Gustafsson, F. Ekdahl, and B. Bergman, "Conjoint analysis : a useful tool in the design process Conjoint analysis : a useful tool in the design," Total Qual. Manag., vol. 10 , no. 3, pp. 327-343, 1999.

[18] P. E. Green and V. Srinivasan, "Conjoint analysis in marketing: new developments with implications for research and practice," J. Mark., vol. 54, no. 4, p. 3, Oct. 1990.

[19] H. Moskowitz and K. J. Kim, "QFD Optimizer: a novice friendly quality function deployment decision support system for optimizing product designs," Comput. Ind. Eng., vol. 32, no. 3, pp. 641-655, Jul. 1997.

[20] R. Y. K. Fung, K. Popplewell, and J. Xie, "An intelligent hybrid system for customer requirements analysis and product attribute targets determination," Int. J. Prod. Res., vol. 36, no. 1, pp. 13-34, Jan. 1998.

[21] X. Du, J. Jiao, and M. M. Tseng, "Understanding customer satisfaction in product customization,” Int. J. Adv. Manuf. Technol., vol. 31, no. 3-4, pp. 396-406, 2006.

[22] J. Jiao and C.-H. Chen, "Customer requirement management in product development: a review of research issues," Concurr. Eng., vol. 14, no. 3, pp. 173185, Sep. 2006.

[23] C. K. Prahalad and V. Ramaswamy, The Future of Competition: Co-Creating Unique Value with Customers. Boston, MA: Harvard Business School Press, 2004.

[24] P. G. Smith, Flexible Product Development: Bring Agility for Changing Markets. San Francisco, CA: Josssey-Bass, 2007.

[25] V. Shaw and C. T. Shaw, "Conflict between engineers and marketers," Ind. Mark. Manag., vol. 27, no. 4, pp. 279-291, Jul. 1998.

[26] S. M. Keaveney, "The blame game: an attribution theory approach to marketerengineer conflict in high-technology companies," Ind. Mark. Manag., vol. 37, no. 6, pp. 653-663, Aug. 2008.

[27] S. Pötzl, T. Kohr, and M. Zollenkop, "Success factors and levers for best practice in innovation management," in Operations Excellence, London: Palgrave Macmillan UK, 2008, pp. 61-78.

[28] A. Coman and B. Ronen, "Icarus' predicament: Managing the pathologies of overspecification and overdesign," Int. J. Proj. Manag., vol. 28, no. 3, pp. 237244, 2010.

[29] G. Castellion and S. K. Markham, "Perspective: New product failure rates: Influence of Argumentum ad populum and self-interest," J. Prod. Innov. Manag., vol. 30, no. 5, pp. 976-979, 2013.

[30] E. von Hippel, "Democratizing innovation: the evolving phenomenon of user innovation," Int. J. Innov. Sci., vol. 1, no. 1, pp. 29-40, Mar. 2009.

[31] C. Prahalad and V. Ramaswamy, "Co-opting customer competence," Harv. Bus. Rev., vol. 78, no. 1, pp. 79-90, 2000.

[32] R. B. Woodruff, "Customer value: The next source for competitive advantage," $J$. Acad. Mark. Sci., vol. 25, no. 2, pp. 139-153, Mar. 1997.

[33] D. J. Flint, "Compressing new product success-to-success cycle time deep customer value understanding and idea generation," Ind. Mark. Manag., vol. 31, pp. 305-315, 2002.

[34] K. Brockhoff, "Customers' perspectives of involvement in new product development," Int. J. Technol. Manag., vol. 26, no. 5/6, p. 464, 2003. 
[35] E. B.-N. Sanders, "Converging perspectives: product development research for the 1990s," Des. Manag. J. (Former Ser., vol. 3, no. 4, pp. 49-54, Jun. 2010.

[36] F. Schweitzer, "Integrating customers at the front end of innovation," in Management of the Fuzzy Front End of Innovation, O. Gassmann and F. Schweitzer, Eds. Cham, Switzerland: Springer International Publishing, 2014, pp. 31-48.

[37] K. Vredenburg, S. Isensee, and C. Righi, User-Centered Design: An Integrated Approach. Upper Saddle River, NJ: Prentice Hall PTR, 2002.

[38] K. E. Gruner and C. Homburg, "Does customer interaction enhance new product success?," J. Bus. Res., vol. 49, no. 1, pp. 1-14, 2000.

[39] E. von Hippel, "Successful industrial products from customer ideas," J. Mark., vol. 42, no. 1, p. 39, Jan. 1978.

[40] C. Hienerth, C. Lettl, and P. Keinz, "Synergies among producer firms, lead users, and user communities: the case of the lego producer-user ecosystem," J. Prod. Innov. Manag., vol. 31, no. 4, pp. 848-866, 2014.

[41] M. Bogers, A. Afuah, and B. Bastian, "Users as innovators: a review, critique, and future research directions," J. Manage., vol. 36, no. 4, pp. 857-875, Jan. 2010.

[42] D. Dougherty, “The maker movement," Innovations, vol. 7, no. 3, pp. 11-14, 2012.

[43] K. Peppler and S. Bender, "Maker movement spreads innovation one project at a time," Phi Delta Kappan, vol. 95, no. 3, p. 6, 2013.

[44] R. W. Veryzer and B. Borja de Mozota, "The impact of user-oriented design on new product development: an examination of fundamental relationships," J. Prod. Innov. Manag., vol. 22, no. 2, pp. 128-143, Mar. 2005.

[45] C. K. Prahalad and V. Ramaswamy, "The new frontier of experience innovation," MIT Sloan Manag. Rev., vol. 44, no. 4, pp. 12-18, 2003.

[46] G. Venturi, J. Troost, and T. Jokela, "People, Organizations, and Processes: an inquiry into the adoption of user-centered design in industry," Int. J. Hum. Comput. Interact., vol. 21, no. 2, pp. 219-238, 2006.

[47] F. Schweitzer, M. Palmié, and O. Gassmann, "Beyond listening: the distinct effects of proactive versus responsive customer orientation on the reduction of uncertainties at the fuzzy front end of innovation," $R \& D$ Manag., pp. 1-18, Nov. 2016.

[48] D. L. Johnstone, "Engineering contributions to the evolution of management practice," IEEE Transactions on Engineering Management, vol. 36, no. 2. pp. 105-113, 1989.

[49] P. J. Denning and R. Dunham, "The Profession of IT: the core of the third-wave professional," Commun. ACM, vol. 44, no. 11, pp. 21-25, Nov. 2001.

[50] H. Sakano and M. Kosaka, "A consideration on success factors in product innovation from the viewpoints of value co-creation with customers," 2013 10th Int. Conf. Serv. Syst. Serv. Manag. - Proc. ICSSSM 2013, no. 24510186, pp. 5359, 2013.

[51] N. E. Coviello et al., "Creating major innovations with customers: insights from small and young technology firms," J. Mark., vol. 76, no. November, pp. 15477185, 2012. 
[52] E. B.-N. Sanders and P. J. Stappers, "Co-creation and the new landscapes of design," CoDesign, vol. 4, no. 1, pp. 5-18, 2008.

[53] R. K. Moenaert, A. De Meyer, W. E. Souder, and D. Deschoolmeester, "R\&D/marketing communication during the fuzzy front-end," IEEE Trans. Eng. Manag., vol. 42, no. 3, pp. 243-258, 1995.

[54] J. Xie, X. M. Song, and A. Stringfellow, "Interfunctional conflict, conflict resolution styles, and new product success: a four-culture comparison," Manage. Sci., vol. 44, no. 12-Part-2, pp. S192-S206, 1998.

[55] R. Calantone and G. Rubera, "When should rd\&e and marketing collaborate? the moderating role of exploration-exploitation and environmental uncertainty," $J$. Prod. Innov. Manag., vol. 29, no. 1, pp. 144-157, Jan. 2012.

[56] D. Leonard-Barton, "Core capabilities and core rigidities: a paradox in managing new product development," Strateg. Manag. J., vol. 13, no. S1, pp. 111-125, Jan. 1992.

[57] R. W. Ruekert and O. C. Walker, "Interactions between marketing and R\&D departments in implementing different business strategies," Strateg. Manag. J., vol. 8, no. 3, pp. 233-248, May 1987.

[58] P.-K. Lam and K.-S. Chin, "Identifying and prioritizing critical success factors for conflict management in collaborative new product development," Ind. Mark. Manag., vol. 34, no. 8, pp. 761-772, Nov. 2005.

[59] J. Mohr, S. Sengupta, and S. Slater, Marketing of High-Technology Products and Innovations, 3rd ed. Upper Saddle River, NJ: Prentice Hall, 2010.

[60] G. L. Rein, "From experience: creating synergy between marketing and research and development," J. Prod. Innov. Manag., vol. 21, no. 1, pp. 33-43, Jan. 2004.

[61] A. K. Gupta, S. P. Raj, and D. Wilemon, "A Model for Studying R\&D. Marketing Interface in the Product Innovation Process," J. Mark., vol. 50, no. 2, p. 7, Apr. 1986.

[62] C. T. Shaw, V. Shaw, and M. Enke, "Relationships between engineers and marketers within new product development," Eur. J. Mark., vol. 38, no. 5/6, pp. 694-719, May 2004.

[63] Y. Haik and T. M. Shahin, Engineering Design Process, 2nd ed. Stamford, CT: Cengage Learning, 2011.

[64] R. Schmidt, "The implementation of simultaneous engineering in the stage of product concept development: A process orientated improvement of quality function deployment," Eur. J. Oper. Res., vol. 100, no. 2, pp. 293-314, Jul. 1997.

[65] I. Van De Poel, "Methodological problems in QFD and directions for future development," Res. Eng. Des., vol. 18, no. 1, pp. 21-36, 2007.

[66] Z. Zhang and X. Chu, "A new integrated decision-making approach for design alternative selection for supporting complex product development," Int. J. Comput. Integr. Manuf., vol. 22, no. 3, pp. 179-198, 2009.

[67] X. X. Shen, K. C. Tan, and M. Xie, "The implementation of quality function deployment based on linguistic data," J. Intell. Manuf., vol. 12, no. 1, pp. 65-75, 2001.

[68] J. J. Michalek, P. Ebbes, F. Adigüzel, F. M. Feinberg, and P. Y. Papalambros, "Enhancing marketing with engineering: optimal product line design for heterogeneous markets," Int. J. Res. Mark., vol. 28, no. 1, pp. 1-12, 2011. 
[69] M. C. Lin, C. C. Wang, M. S. Chen, and C. A. Chang, "Using AHP and TOPSIS approaches in customer-driven product design process," Comput. Ind., vol. 59, no. 1, pp. 17-31, 2008.

[70] Z. Ayağ and R. G. Özdemir, "A hybrid approach to concept selection through fuzzy analytic network process," Comput. Ind. Eng., vol. 56, no. 1, pp. 368-379, 2009.

[71] R. Srinivasan, G. L. Lilien, A. Rangaswamy, G. M. Pingitore, and D. Seldin, "The total product design concept and an application to the auto market," J. Prod. Innov. Manag., vol. 29, pp. 3-20, 2012.

[72] S. Nadkarni and P. P. Shenoy, "Bayesian network approach to making inferences in causal maps," Eur. J. Oper. Res., vol. 128, no. 3, pp. 479-498, 2001.

[73] H. H. Schröder and A. J. M. Jetter, "Integrating market and technological knowledge in the fuzzy front end: an FCM-based action support system," Int. J. Technol. Manag., vol. 26, no. 5/6, p. 517, 2003.

[74] W. Yan, C. H. Chen, and M. D. Shieh, "Product concept generation and selection using sorting technique and fuzzy c-means algorithm," Comput. Ind. Eng., vol. 50, no. 3, pp. 273-285, 2006.

[75] G. Pahl, B. Wolfgang, J. Feldhusen, and K.-H. Grote, Engineering Design: A systematic Approach, 3rd ed. New York: Springer-Verlag, 2007.

[76] A. M. King and S. Sivaloganathan, "Development of a methodology for concept selection in flexible design strategies," J. Eng. Des., vol. 10, no. 4, pp. 329-349, Dec. 1999.

[77] A. Griffin and J. R. Hauser, "The voice of the customer," Mark. Sci., vol. 12, no. 1, pp. 1-27, Feb. 1993.

[78] T. A. W. Jarratt, C. M. Eckert, N. H. M. Caldwell, and P. J. Clarkson, "Engineering change: an overview and perspective on the literature," Res. Eng. Des., vol. 22, no. 2, pp. 103-124, 2011.

[79] E. C. Y. Koh, N. H. M. Caldwell, and P. J. Clarkson, "A method to assess the effects of engineering change propagation," Res. Eng. Des., vol. 23, no. 4, pp. 329-351, 2012.

[80] B. M. Kennedy, D. K. Sobek, and M. N. Kennedy, "Reducing rework by applying set-based practices early in the systems engineering process," Syst. Eng., vol. 17, no. 3, pp. 278-296, Sep. 2014.

[81] R. Ganguli, "Fuzzy cognitive maps for structural damage detection," in Fuzzy Cognitive Maps for Applied Sciences and Engineering, E. I. Papageorgiou, Ed. Springer Berlin Heidelberg, 2014, pp. 267-290.

[82] M. E. Porter and J. E. Heppelmann, "How smart, connected products are transforming competition," Harv. Bus. Rev., vol. 92, no. 11, pp. 64-88, 2014.

[83] D. Turner, "Different: Inside (sort of) Apple's industrial-design machine," MIT Technol. Rev., vol. 110, no. 3, pp. 54-59, 2007.

[84] D. Norman and B. Tognazzini, "How Apple is giving design a bad name," FASTCOMPANY, 2015.

[85] K. Lovelace, D. L. Shapiro, and L. R. Weingart, "Maximizing cross-functional new product teams' innovativeness and constraint adherence: A conflict communications perspective," Acad. Manag. J., vol. 44, no. 4, pp. 779-793, Aug. 2001. 
[86] K. B. Kahn, "Functional, multifunctional, and cross-functional: considerations for marketing management," J. Mark. Theory Pract., vol. 17, no. 1, pp. 75-84, 2009.

[87] R. C. McNally, M. B. Akdeniz, and R. J. Calantone, "New product development processes and new product profitability: Exploring the mediating role of speed to market and product quality," J. Prod. Innov. Manag., vol. 28, no. 1, pp. 63-77, 2011.

[88] R. I. Campbell et al., "Design evolution through customer interaction with functional prototypes," J. Eng. Des., vol. 18, no. 6, pp. 617-635, 2007.

[89] E. von Hippel, Democratizing innovation. Cambridge, MA: MIT Press, 2005.

[90] C. Hienerth, E. von Hippel, and M. Berg Jensen, "User community vs. producer innovation development efficiency: A first empirical study," Res. Policy, vol. 43, no. 1, pp. 190-201, 2014.

[91] S. F. Slater and J. C. Narver, "Customer-led and market-oriented: let's not confuse the two," vol. 1006, Strateg. Manag. J., no. January 1997, pp. 1001-1006, 1998.

[92] U. Brentani and S. E. Reid, "The fuzzy front-end of discontinuous innovation: insights for research and management," J. Prod. Innov. Manag., vol. 29, no. 1, pp. 70-87, Jan. 2012.

[93] M. Schoonmaker, E. Carayannis, and P. Rau, "The role of marketing activities in the fuzzy front end of innovation: a study of the biotech industry," J. Technol. Transf., vol. 38, no. 6, pp. 850-872, Dec. 2012.

[94] E. Stevens, "Fuzzy front-end learning strategies: exploration of a high-tech company," Technovation, vol. 34, no. 8, pp. 431-440, 2014.

[95] R. Sperry and A. Jetter, "Theoretical framework for managing the front end of innovation under uncertainty," PICMET '09 - 2009 Portl. Int. Conf. Manag. Eng. Technol., pp. 2021-2028, Aug. 2009.

[96] A. Khurana and S. Rosenthal, "Integrating the fuzzy front end of new product development," Sloan Manage. Rev., vol. 38, no. 2, pp. 103-120, 1997.

[97] I. Alam, "Removing the fuzziness from the fuzzy front-end of service innovations through customer interactions," Ind. Mark. Manag., vol. 35, no. 4, pp. 468-480, May 2006.

[98] R. Cooper, S. Edgett, and E. Kleinschmidt, "Portfolio management for new product development: results of an industry practices study," $R$ D Manag., vol. 31, no. 4, pp. 361-380, Oct. 2001.

[99] P. G. Smith and D. G. Reinertsen, Design products in half the time. New York, NY: Van Nostrand Reinhold, 1991.

[100] J. Kim and D. Wilemon, "Focusing the fuzzy front-end in new product development," R\&D Manag., vol. 32, no. 4, pp. 269-279, Sep. 2002.

[101] R. G. Cooper and E. J. Kleinschmidt, "Determinants of timeliness in product development," J. Prod. Innov. Manag., vol. 11, no. 5, pp. 381-396, Nov. 1994.

[102] B. Verworn, C. Herstatt, and A. Nagahira, "The fuzzy front end of Japanese new product development projects: impact on success and differences between incremental and radical projects," $R \& D$ Manag., vol. 38, no. 1, pp. 1-19, Dec. 2007.

[103] B. Verworn, "A structural equation model of the impact of the 'fuzzy front end' on the success of new product development," Res. Policy, vol. 38, no. 10, pp. 1571$1581,2009$. 
[104] R. G. Cooper, "Predevelopment activities determine new product success," Ind. Mark. Manag., vol. 17, no. 3, pp. 237-247, Aug. 1988.

[105] C. Herstatt and B. Verworn, "The "fuzzy front end' of innovation," in Bringing Technology and Innovation into the Boardroom, London: Palgrave Macmillan UK, 2004, pp. 347-372.

[106] M. Backman, S. Börjesson, and S. Setterberg, "Working with concepts in the fuzzy front end: exploring the context for innovation for different types of concepts at Volvo Cars," R\&D Manag., vol. 37, no. 1, Jan. 2007.

[107] M. Kurkkio, J. Frishammar, and U. Lichtenthaler, "Where process development begins: A multiple case study of front end activities in process firms,"

Technovation, vol. 31, no. 9, pp. 490-504, Sep. 2011.

[108] S. Chang, C. Chen, and S. Wey, "Conceptualizing, assessing, and managing frontend fuzziness in innovation/NPD projects," $R \& D$ Manag., vol. 37, no. 5, pp. 469478, Nov. 2007.

[109] P. A. Koen et al., "Fuzzy front end: effective methods, tools, and techniques," in The PDMA Toolbook, 2002, pp. 5-35.

[110] P. Koen et al., "Providing clarity and a common language to the "fuzzy front end,"” Res. Manag., vol. 44, no. 2, pp. 46-55, 2001.

[111] L. Hannola, J. Friman, and J. Niemimuukko, "Application of agile methods in the innovation process," Int. J. Bus. Innov. Res., vol. 7, no. 1, p. 84, 2013.

[112] P. Kettunen, "Adopting key lessons from agile manufacturing to agile software product development-a comparative study," Technovation, vol. 29, no. 6-7, pp. 408-422, 2009.

[113] B. Guérineau, L. Rivest, M. Bricogne, and A. Durupt, "Agile and project-planned methods in multidisciplinary product design," in Product Lifecycle Management for Digital Transformation of Industries. PLM 2016. IFIP Advances in Information and Communication Technology, 2016, pp. 108-118.

[114] R. G. Cooper and A. F. Sommer, "The agile-stage-gate hybrid model: a promising new approach and a new research opportunity," J. Prod. Innov. Manag., vol. 33, no. 5, pp. 513-526, 2016.

[115] S. F. Slater, J. J. Mohr, and S. Sengupta, "Radical product innovation capability: Literature review, synthesis, and illustrative research propositions," J. Prod. Innov. Manag., vol. 31, no. 3, pp. 552-566, 2014.

[116] S. Strambach and B. Klement, "Cumulative and combinatorial micro-dynamics of knowledge: the role of space and place in knowledge integration," Eur. Plan. Stud., vol. 20, no. 11, pp. 1843-1866, 2012.

[117] A. Griffin and J. R. Hauser, "Integrating r\&d and marketing: a review and analysis of the literature," J. Prod. Innov. Manag., vol. 13, no. 3, pp. 191-215, 1996.

[118] E. von Hippel, "The dominant role of users in the scientific instrument innovation process," Res. Policy, vol. 5, no. 3, pp. 212-239, Jul. 1976.

[119] M. H. Zack, "If managing knowledge is the solution, then what's the problem?," in Knowledge Management and Business Model Innovation, IGI Global, 2001, pp. 16-36.

[120] J. Frishammar, H. Floren, and J. Wincent, "Beyond managing uncertainty: insights from studying equivocality in the fuzzy front end of product and process 
innovation projects," IEEE Trans. Eng. Manag., vol. 58, no. 3, pp. 551-563, Aug. 2011.

[121] D. E. Rosen, J. E. Schroeder, and E. F. Purinton, "Marketing high tech products : lessons in customer focus from the marketplace," Acad. Mark. Sci. Rev., vol. 1998, no. 06, 1998.

[122] J. Kim and D. Wilemon, "Strategic issues in managing innovation's fuzzy frontend," Eur. J. Innov. Manag., vol. 5, no. 1, pp. 27-39, 2002.

[123] A. Jetter, F. Albar, and R. Sperry, "The practice of project management in product development: insights from the literature and cases in high-tech," 2016.

[124] C. Andriopoulos, M. Gotsi, M. W. Lewis, and A. E. Ingram, "Turning the sword: how npd teams cope with front-end tensions," J. Prod. Innov. Manag., vol. 35, no. 3, pp. 427-445, 2018.

[125] P. Belliveau, A. Griffin, and S. Somermeyer, Eds., The PDMA Toolbook for New Product Development. New York, NY: John Wiley \& Sons, 2002.

[126] X. Koufteros, M. Vonderembse, and J. Jayaram, "Internal and external integration for product development: the contingency effects of uncertainty, equivocality, and platform strategy," Decis. Sci., vol. 36, no. 1, pp. 97-133, Feb. 2005.

[127] M. Song and M. M. Montoya-Weiss, "The effect of perceived technological uncertainty on japanese new product development," Acad. Manag. J., vol. 44, no. 1, pp. 61-80, Feb. 2001.

[128] J. D. Sherman, D. Berkowitz, and W. E. Souder, "New product development performance and the interaction of cross-functional integration and knowledge management," J. Prod. Innov. Manag., vol. 22, no. 5, pp. 399-411, 2005.

[129] X. Lin and R. Germain, "Antecedents to customer involvement in product development: Comparing US and Chinese firms," Eur. Manag. J., vol. 22, no. 2, pp. 244-255, 2004.

[130] R. L. Daft and R. H. Lengel, "Organizational information requirements, media richness and structural design,” Manage. Sci., vol. 32, no. 5, pp. 554-571, May 1986.

[131] D. K. Berlo, The process of communication: An introduction to theory and practice. Holt, Rinehart and Winston, 1960.

[132] S. J. Hoch and J. Deighton, "Managing what consumers learn from experience," J. Mark., vol. 53, no. 2, p. 1, Apr. 1989.

[133] M. L. Knapp and J. A. Hall, Nonverbal communication in human interaction, 8th ed. Boston, MA: Wadsworth Gengage Learning, 2014.

[134] B. Downe-Wamboldt, "Content analysis: method, applications, and issues.," Health Care Women Int., vol. 13, no. 3, pp. 313-21, 1992.

[135] G. Pérez-Bustamante, "Knowledge management in agile innovative organisations," J. Knowl. Manag., vol. 3, no. 1, pp. 6-17, Mar. 1999.

[136] B. Nooteboom, "Learning by interaction: absorptive capacity, cognitive distance and governance," J. Manag. Gov., vol. 4, no. 1, pp. 69-92, 2000.

[137] W. M. Cohen and D. A. Levinthal, "Absorptive capacity: a new perspective on learning and innovation," Adm. Sci. Q., vol. 35, no. 1, p. 128, Mar. 1990.

[138] S. A. Zahra and G. George, "Absorptive capacity: a review, reconceptualization, and extension," Acad. Manag. Rev., vol. 27, no. 2, pp. 185-203, Apr. 2002. 
[139] H. W. Chesbrough, Open Innovation: The New Imperative for Creating And Profiting from Technology. Boston: Harvard Business Review Press, 2003.

[140] K. Petersen and C. Wohlin, "A comparison of issues and advantages in agile and incremental development between state of the art and an industrial case," J. Syst. Softw., vol. 82, no. 9, pp. 1479-1490, Sep. 2009.

[141] C. Argyris and D. A. Schön, Organizational Learning: A Theory of Action Perspective. Reading, MA: Addison-Wesley, 1978.

[142] D. H. Kim, "The link between individual and organizational learning," Sloan Manage. Rev., vol. 35, no. 1, pp. 37-50, 1993.

[143] N. a. Jones, H. Ross, T. Lynam, P. Perez, and A. Leitch, "Mental model an interdisciplinary synthesis of theory and methods," Ecol. Soc., vol. 16, no. 1, pp. 46-46, 2011.

[144] P. van den Bossche, W. Gijselaers, M. Segers, G. Woltjer, and P. Kirschner, "Team learning: building shared mental models," Instr. Sci., vol. 39, no. 3, pp. 283-301, 2011.

[145] S. Mohammed and B. C. Dumville, "Team mental models in a team knowledge framework: expanding theory and measurement across disciplinary boundaries," $J$. Organ. Behav., vol. 22, no. 2, pp. 89-106, 2001.

[146] E. Blickensderfer, J. A. Cannon-Bowers, and E. Salas, "Theoretical bases for team self-correction: fostering shared mental models," Adv. Interdiscip. Stud. Work teams, vol. 4, no. 1, pp. 249-279, 1997.

[147] D. M. Kennedy, "Team creative processes: The importance of complementary and shared mental models," in Proceedings of the Annual Hawaii International Conference on System Sciences, 2011, pp. 1-10.

[148] M. Cadwallader, "Problems in Cognitive Distance," Environ. Behav., vol. 11, no. 4, pp. 559-576, Dec. 1979..

[149] D. B. MacKay, R. W. Olshavsky, and G. Sentell, "Cognitive maps and spatial behavior of consumers," Geogr. Anal., vol. 7, no. 1, pp. 19-33, 1975.

[150] D. R. Montello, "The measurement of cognitive distance: methods and construct validity," J. Environ. Psychol., vol. 11, no. 2, pp. 101-122, 1991.

[151] S. Wuyts, M. G. Colombo, S. Dutta, and B. Nooteboom, "Empirical tests of optimal cognitive distance," J. Econ. Behav. Organ., vol. 58, no. 2, pp. 277-302, 2005.

[152] V. Gilsing, B. Nooteboom, W. Vanhaverbeke, G. Duysters, and A. van den Oord, "Network embeddedness and the exploration of novel technologies: technological distance, betweenness centrality and density," Res. Policy, vol. 37, no. 10, pp. 1717-1731, 2008.

[153] E. Enkel and O. Gassmann, "Creative imitation: Exploring the case of crossindustry innovation," $R \& D$ Manag., vol. 40, no. 3, pp. 256-270, 2010.

[154] A. Muscio and A. Pozzali, "The effects of cognitive distance in university-industry collaborations: Some evidence from Italian universities," J. Technol. Transf., vol. 38, no. 4, pp. 486-508, 2013.

[155] B. Nooteboom, W. Van Haverbeke, G. Duysters, V. Gilsing, and A. van den Oord, "Optimal cognitive distance and absorptive capacity," Res. Policy, vol. 36, no. 7, pp. 1016-1034, 2007. 
[156] R. Sethi, D. C. Smith, and W. Park, "How to kill a team' s creativity," Harv. Bus. Rev., vol. 80, no. 8, pp. 16-17, 2002.

[157] B. Nooteboom, W. Van Haverbeke, G. Duysters, V. Gilsing, and A. van den Oord, "Optimal cognitive distance and absorptive capacity," Res. Policy, vol. 36, no. 7, pp. 1016-1034, Sep. 2007.

[158] P. L. Link, "Keys to new product success and failure," Ind. Mark. Manag. 16, vol. 16, no. 2, pp. 109-118, 1987.

[159] R. Cooper, "Dimensions of new industrial product success and failure," J. Mark., vol. 43, no. 3, pp. 93-103, 1979.

[160] R. Schwientek, A. Schmidt, and Roland Berger Strategy Consultants, Operations Excellence: Smart Solutions for Business Success. Basingstoke, NY: Palgrave Macmillan, 2008.

[161] V. Krishnan and K. T. Ulrich, "Product development decisions: a review of the literature," Manage. Sci., vol. 47, no. 1, pp. 1-21, 2001.

[162] R. Y. K. Fung, Shouju Ren, and Jinxing Xie, "The prioritisation of attributes in customer requirement management," in 1996 IEEE International Conference on Systems, Man and Cybernetics. Information Intelligence and Systems (Cat. No.96CH35929), 1996, vol. 2, pp. 953-958.

[163] M. Vriens and F. Ter Hofstede, "Linking attributes, benefits, and consumer values," Mark. Res., vol. 12, no. Fall, pp. 5-10, 2000.

[164] M. Crawford and A. Di Benedetto, New Products Management, 10th ed. New York, NY: McGraw-Hill/Irwin, 2011.

[165] M. L. Shillito, Acquiring, Processing, and Deploying Voice of the Customer. Bova Raton, FL: CRC Press LLC, 2001.

[166] V. A. Zeithaml, "Consumer perceptions of price, quality, and value: a means-end model and synthesis of evidence," J. Mark., vol. 52, no. 3, p. 2, Jul. 1988.

[167] C. Homburg, M. Schwemmle, and C. Kuehnl, "New product design: concept, measurement, and consequences," J. Mark., vol. 79, no. 3, pp. 41-56, May 2015.

[168] J. Shaw, J. Giglierano, and J. Kallis, "Marketing complex technical products: the importance of intangible attributes," Ind. Mark. Manag., vol. 18, no. 1, pp. 45-53, 1989.

[169] T. Levitt, "Marketing success through differentiation- of anything," Harv. Bus. Rev., no. January, pp. 83-92, 1980.

[170] S. Wang, A. Wang, and Y. Wang, "Structural attributes and evolution features of products," LISS 2014, pp. 1477-1482, 2015.

[171] N. Kano, N. Seraku, F. Tsuji, and S. Takahashi, "Attractuve quality and must be quality,” J. Japanese Soc. Qual. Control, vol. 14, no. 2, pp. 39-48, 1984.

[172] E. MacDonald, M. Backsell, R. Gonzalez, and P. Papalambros, "The kano method's imperfections, and implications in product decision theory," Proc. 2006 Int. Des. Res. Symp., pp. 1-12, 2006.

[173] C. C. Chen and M. C. Chuang, "Integrating the Kano model into a robust design approach to enhance customer satisfaction with product design," Int. J. Prod. Econ., vol. 114, no. 2, pp. 667-681, 2008.

[174] V. Srinivasan, W. S. Lovejoy, and D. Beach, "Integrated product design for marketability and manufacturing," J. Mark. Res., vol. 34, no. 1, pp. 154-163, Feb. 1997. 
[175] A. M. Tybout and J. R. Hauser, "A marketing audit using a conceptual model of consumer behavior: application and evaluation," J. Mark., vol. 45, no. 3, p. 82, Jan. 1981.

[176] R. W. Veryzer and B. B. De Mozota, "The impact of user-oriented design on new product development: An examination of fundamental relationships," J. Prod. Innov. Manag., vol. 22, no. 2, pp. 128-143, 2005.

[177] A. P. O. S. Vermeeren, E. L.-C. Law, V. Roto, M. Obrist, J. Hoonhout, and K. Väänänen-Vainio-Mattila, "User experience evaluation methods," in Proceedings of the 6th Nordic Conference on Human-Computer Interaction Extending Boundaries - NordiCHI '10, 2010, p. 521.

[178] M. Hassenzahl, "User experience (UX): towards an experiential perspective on product quality," Proc. 20th Int. Conf. Assoc. Francoph. d'Interaction HommeMachine - IHM '08, pp. 11-15, 2008.

[179] A. Bruseberg and D. McDonagh-Philp, "New product development by eliciting user experience and aspirations," Int. J. Human- ..., vol. 55, p. 435 \}452, 2001.

[180] S. Schütte, "Engineering Emotional Values in Product Design -Kansei Engineering in Development," Ph.D. dissertation, Dept. Manage. Eng., Linköping Univ., Linköping, Sweden, 2005.

[181] P. Desmet, K. Overbeeke, and S. Tax, "Designing products with added emotional value: development and application of an approach for research through design," Des. J., vol. 4, no. April, pp. 32-47, 2001.

[182] H. M. Khalid, "Customer emotional needs in product design," Concurr. Eng., vol. 14, no. 3, pp. 197-206, 2006.

[183] T. Lageat, S. Czellar, and G. Laurent, "Hedonic attributes engineering of luxury: to generate perceptions of an everyday consumer sound perception," vol. 14, no. 2 , pp. 97-109, 2003.

[184] H. Yanagisawa, A. Kataoka, T. Murakami, K. Ohtomi, and R. Hosaka, "Extraction of latent emotional factors by analyzing human sensitivity towards unexplored design," Iced'09, vol. 7, no. August, pp. 13-24, 2009.

[185] M. Nagamachi, "Kansei engineering as a powerful consumer-oriented technology for product development," Appl. Ergon., vol. 33, no. 3, pp. 289-294, 2002.

[186] B. El-Haik and K. Yang, "The components of complexity in engineering design," IIE Trans., vol. 31, no. 10, pp. 925-934, Oct. 1999.

[187] F. Ameri, J. D. Summers, G. M. Mocko, and M. Porter, "Engineering design complexity: an investigation of methods and measures," Res. Eng. Des., vol. 19, no. 2-3, pp. 161-179, 2008.

[188] H. Kandjani and P. Bernus, "Engineering self-designing enterprises as complex systems using extended axiomatic design theory," IFAC Proc. Vol., vol. 44, no. 1, pp. 11943-11948, Jan. 2011.

[189] A. J. M. Jetter, "Educating the guess: strategies, concepts and tools for the fuzzy front end of product development," in PICMET '03: Portland International Conference on Management of Engineering and Technology Technology Management for Reshaping the World, 2003., 2003, pp. 261-273.

[190] S. E. Reid and U. De Brentani, "The fuzzy front end of new product development for discontinuous innovations: a theoretical model," J. Prod. Innov. Manag., vol. 21, no. 2, pp. 170-184, 2004. 
[191] H. Akbar and N. Tzokas, “An exploration of new product development's front-end knowledge conceptualization process in discontinuous innovations," $\mathrm{Br} . \mathrm{J}$. Manag., vol. 24, no. 2, pp. 245-263, 2013.

[192] A. M. Davis and A. M. Hickey, "Requirements researchers: do we practice what we preach?," Requir. Eng., vol. 7, no. 2, pp. 107-111, Jun. 2002.

[193] J. D. Weinrauch and R. Anderson, "Conflicts between engineering and marketing units," Ind. Mark. Manag., vol. 11, no. 4, pp. 291-301, Oct. 1982.

[194] P. R. Carlile, "A pragmatic view of knowledge and boundaries: boundary objects in new product development," Organ. Sci., vol. 13, no. 4, pp. 442-455, Aug. 2002.

[195] K.Frazier "DOE secretary praises Sandia: Spencer Abraham's remarks to Sandians," Sandia LabNews, Albuquerque, New Mexico, p. 5, 10-Jan-2003.

[196] L.-K. Chan and M.-L. Wu, Quality function deployment: A literature review, vol. 143, no. 3. 2002.

[197] T. U. Pimmler and S. D. Eppinger, "Integration analysis of product decompositions," in ASME 1994 International Design Engineering Technical Conferences \& 6th Design Theory and Methodology Conference. Minneapolis, USA, 11-14 September 1994, 1994, no. September, pp. 343-351.

[198] P. Vermaas and P. Garbacz, "Functional decomposition and mereology in engineering," in Philosophy of Technology and Engineering Sciences, vol. 9, D. M. Gabbay, P. Thagard, J. Woods, and A. W. M. Meijers, Eds. Amsterdam, Netherlands: Elsevier, 2009, pp. 235-271.

[199] S. D. Eppinger, "A planning method for integration of large-scale engineering systems," in International Conference on Engineering Design, 1997, pp. 199-204.

[200] N. P. Suh, The Principles of Design. New York, NY: Oxford University Press, 1990.

[201] N. P. Suh, Axiomatic design. New York, NY: Oxford University Press, 2001.

[202] D. Tang, G. Zhang, and S. Dai, "Design as integration of axiomatic design and design structure matrix," Robot. Comput. Integr. Manuf., vol. 25, no. 3, pp. 610619, 2009.

[203] T. Randall, C. Terwiesch, and K. T. Ulrich, "Research note--user design of customized products," Mark. Sci., vol. 26, no. 2, pp. 268-280, 2007.

[204] Z. Ayağ and R. G. Özdemir, "An analytic network process-based approach to concept evaluation in a new product development environment," J. Eng. Des., vol. 18, no. 3, pp. 209-226, Jun. 2007.

[205] K. Chin, D. Tang, J. Yang, S. Y. Wong, and H. Wang, “Assessing new product development project risk by Bayesian network with a systematic probability generation methodology," Expert Syst. Appl., vol. 36, no. 6, pp. 9879-9890, 2009.

[206] O. Kulak and C. Kahraman, "Multi-attribute comparison of advanced manufacturing systems using fuzzy vs. crisp axiomatic design approach," Int. J. Prod. Econ., vol. 95, no. 3, pp. 415-424, 2005.

[207] B. Hillebrand and W. G. Biemans, "Links between internal and external cooperation in product development: an exploratory study," J. Prod. Innov. Manag., vol. 21, no. 2, pp. 110-122, Mar. 2004.

[208] R. L. Daft, R. H. Lengel, S. M. Science, and O. D. May, "Organizational information requirements, media richness and structural design," vol. 32, no. 5, pp. 554-571, 1986. 
[209] E. Dahan and V. Srinivasan, "The predictive power of internet-based product concept testing using visual depiction and animation," J. Prod. Innov. Manag., vol. 17, no. 2, pp. 99-109, Mar. 2000.

[210] Z. Rusák, C. Antonya, W. van der Vegte, I. Horváth, and E. Varga, “A new approach to interactive grasping simulation of product concepts in a virtual reality environment," in Volume 2: 27th Computers and Information in Engineering Conference, Parts A and B, 2007, pp. 213-221.

[211] B. Kosko, "Fuzzy cognitive maps," Int. J. Man. Mach. Stud., vol. 24, no. 1, pp. 65-75, Jan. 1986.

[212] B. Kosko, "Hidden patterns in combined and adaptive knowledge networks," Int. J. Approx. Reason., vol. 2, no. 4, pp. 377-393, Oct. 1988.

[213] A. J. M. Jetter, "Fuzzy cognitive maps for engineering and technology management: what works in practice?," in 2006 Technology Management for the Global Future - PICMET 2006 Conference, 2006, no. c, pp. 498-512.

[214] E. I. Papageorgiou, "Review study on fuzzy cognitive maps and their applications during the last decade," in 2011 IEEE International Conference on Fuzzy Systems (FUZZ-IEEE 2011), 2011, no. 1998, pp. 828-835.

[215] J. Aguilar, “A survey about fuzzy cognitive maps papers," Int. J. Comput. Cogn., vol. 3, no. 2, pp. 27-33, 2005.

[216] A. J. M. Jetter and R. C. Sperry, "Fuzzy cognitive maps for product planning: using stakeholder knowledge to achieve corporate responsibility," 2013 46th Hawaii Int. Conf. Syst. Sci., pp. 925-934, Jan. 2013.

[217] R. C. Sperry, "Multi-perspective technology assessment to improve decision making : a novel approach using fuzzy cognitive mapping for a large-scale transmission line upgrade by," Ph.D. dissertation, Dept. Eng. Manage. Portland State Univ., Portland, OR, 2014.

[218] R. Sperry and A. J. Jetter, "Fuzzy cognitive maps to implement corporate social responsibility in product planning : a novel approach," 2012 Proc. PICMET '12 Technol. Manag. Emerg. Technol., pp. 2536-2541, 2012.

[219] B. S. Yoon and A. J. Jetter, "Investigation of different perspectives between developers and customers : robotic vacuum cleaners," in Proceedings of PICMET '14 Conference: Portland International Center for Management of Engineering and Technology; Infrastructure and Service Integration, 2014, pp. 2307-2313.

[220] S. a Gray, E. Zanre, and S. R. J. Gray, "Fuzzy cognitive maps as representations of mental models and group beliefs," Fuzzy Cogn. Maps Appl. Sci. Eng. SE - 2, vol. 54, pp. 29-48, 2014.

[221] M. van Vliet, K. Kok, and T. Veldkamp, "Linking stakeholders and modellers in scenario studies: the use of fuzzy cognitive maps as a communication and learning tool," Futures, vol. 42, no. 1, pp. 1-14, Feb. 2010.

[222] K. Kok, "The potential of Fuzzy Cognitive Maps for semi-quantitative scenario development, with an example from Brazil," Glob. Environ. Chang., vol. 19, no. 1, pp. 122-133, 2009.

[223] R. Satur and L. Zhi-Qiang, "A context-driven intelligent database processing system using object-oriented fuzzy cognitive maps," Int. J. Intell. Syst., vol. 11, pp. 671-689, 1996. 
[224] A. J. M. Jetter, "Elicitation - extracting knowledge from experts," in Knowledge Integration: The Practice of Knowledge Management in Small and Medium Enterprises, A. J. M. Jetter, J. Kraaijenbrink, H.-H. Schröder, and F. Wijnhoven, Eds. Heidelberg, New York: Physica-Verlag, 2006, pp. 65-76.

[225] M. S. Khan and M. Quaddus, "Group decision support using fuzzy cognitive maps for causal reasoning," Gr. Decis. Negot., vol. 13, no. 5, pp. 463-480, 2004.

[226] D. P. Tegarden and S. D. Sheetz, "Group cognitive mapping: a methodology and system for capturing and evaluating managerial and organizational cognition," Omega, vol. 31, no. 2, pp. 113-125, 2003.

[227] A. Jetter and W. Schweinfort, "Building scenarios with fuzzy cognitive maps: an exploratory study of solar energy," Futures, vol. 43, no. 1, pp. 52-66, Feb. 2011.

[228] J. A. Dickerson and B. Kosko, "Virtual worlds asfuzzy cognitive maps," Presence Teleoperators Virtual Environ., vol. 3, no. 2, pp. 173-189, Jan. 1994.

[229] U. Özesmi and S. L. Özesmi, "Ecological models based on people's knowledge: a multi-step fuzzy cognitive mapping approach," Ecol. Modell., vol. 176, no. 1-2, pp. 43-64, Aug. 2004.

[230] M. Schaffernicht and S. N. Groesser, "A comprehensive method for comparing mental models of dynamic systems," Eur. J. Oper. Res., vol. 210, no. 1, pp. 57-67, 2011.

[231] A. K. Tsadiras, "Comparing the inference capabilities of binary, trivalent and sigmoid fuzzy cognitive maps," Inf. Sci. (Ny)., vol. 178, no. 20, pp. 3880-3894, 2008.

[232] J. P. Carvalho and A. B. Tomé, José, "Rule based fuzzy cognitive maps and fuzzy cognitive maps-A Comparative Study," Asterix.Ist.Utl.Pt, pp. 115-119, 1999.

[233] W. S. McCulloch and W. Pitts, "A logical calculus of the ideas immanent in nervous activity," Bull. Math. Biophys., vol. 5, no. 4, pp. 115-133, 1943.

[234] J. Ghaboussi, J. H. Garrett, and X. Wu, "Knowledge-based modeling of material behavior with neural networks," J. Eng. Mech., vol. 117, no. 1, pp. 132-153, Jan. 1991.

[235] A. K. Jain, Jianchang Mao, and K. M. Mohiuddin, "Artificial neural networks: a tutorial," Computer (Long. Beach. Calif)., vol. 29, no. 3, pp. 31-44, Mar. 1996.

[236] M. Amer, "Extending technology roadmap through fuzzy cognitive map-based scenarios: the case of the wind energy sector of pakistan," Portland State University, Portland, OR, 2013.

[237] R. Taber, "Knowledge processing with fuzzy cognitive maps," Expert Syst. Appl., vol. 2, no. 1, pp. 83-87, Jan. 1991.

[238] R. Taber, R. R. Yager, and C. M. Helgason, "Quantization effects on the equilibrium behavior of combined fuzzy cognitive maps," Int. J. Intell. Syst., vol. 22, no. 2, pp. 181-202, 2007.

[239] B. Kosko, Neural networks and fuzzy systems: a dynamical systems approach to machine intelligence/book and disk. Upper Saddle River, NJ: Prentice Hall, 1992.

[240] C. D. Stylios and P. P. Groumpos, "The challenge of modelling supervisory systems using fuzzy cognitive maps," J. Intell. Manuf., vol. 9, no. 4, pp. 339-345, 1998. 
[241] P. P. Groumpos, "Fuzzy cognitive maps: basic theories and their application to complex systems," in Fuzzy cognitive maps, Berlin, Heidelberg: Springer, 2010, pp. 1-22.

[242] M. S. Khan and M. Quaddus, "Group decision support using fuzzy cognitive maps for causal reasoning," Gr. Decis. Negot., vol. 13, no. 5, pp. 463-480, Sep. 2004.

[243] D. Andersen and G. Richardson, "Scripts for group model building," Syst. Dyn. Rev., vol. 13, no. 2, pp. 107-129, 1997.

[244] G. P. Richardson and D. F. Andersen, "Teamwork in group model building," System Dynamics Review, vol. 11, no. 2. pp. 113-137, 1995.

[245] D. F. Andersen, G. P. Richardson, and J. A. M. Vennix, "Group model building: adding more science to the craft," Syst. Dyn. Rev., vol. 13, no. 2, pp. 187-201, 1997.

[246] L. F. Luna-Reyes, I. J. Martinez-Moyano, T. A. Pardo, A. M. Cresswell, D. F. Andersen, and G. P. Richardson, "Anatomy of a group model-building intervention: building dynamic theory from case study research," Syst. Dyn. Rev., vol. 22, no. 4, pp. 291-320, 2006.

[247] A. Voinov and F. Bousquet, "Modelling with stakeholders," Environ. Model. Softw., vol. 25, no. 11, pp. 1268-1281, 2010.

[248] A. J. Jetter, A. Singer, S. Gray, L. Ellsworth, P. Zhang, and O. Laraichi, "Fuzzy cognitive mapping for fire science applications: and introduction for practitioners," Dept. Eng. Manage., Portland State Univ., JFSP 14-2-01-26, Mar. 1, 2017.

[249] L. Macaulay, "Requirements for requirements engineering techniques," in Proceedings of the Second International Conference on Requirements Engineering, 1996, pp. 157-164.

[250] T. R. Adler, "An evaluation of the social perspective in the development of technical requirements," IEEE Trans. Prof. Commun., vol. 43, no. 4, pp. 386-396, 2000.

[251] K. N. Lemon and P. C. Verhoef, "Understanding customer experience throughout the customer journey," J. Mark., vol. 80, no. 6, pp. 69-96, 2016.

[252] A. J. M. Jetter and W. Schweinfort, "Building scenarios with fuzzy cognitive maps: an exploratory study of solar energy," Futures, vol. 43, no. 1, pp. 52-66, Feb. 2011.

[253] A. J. Jetter and K. Kok, "Fuzzy cognitive maps for futures studies - a methodological assessment of concepts and methods," Futures, vol. 61, pp. 45-57, Sep. 2014.

[254] Y. Alizadeh and A. Jetter, "Content analysis using fuzzy cognitive map (FCM): a guide to capturing causal relationships from secondary sources of data," in 2017 Portland International Conference on Management of Engineering and Technology (PICMET), 2017, no. M, pp. 1-11.

[255] S. A. Gray, S. Gray, L. J. Cox, and S. Henly-Shepard, "Mental modeler: a fuzzylogic cognitive mapping modeling tool for adaptive environmental management," Proc. Annu. Hawaii Int. Conf. Syst. Sci., pp. 965-973, 2013.

[256] FCMapper. (2016), S. Turney and M. Bachhofer.

[257] fcm. (2017) Z. Dikopoulou and E. Papageorgiou.

[258] S. Thomke and D. Reinertsen, "Six myths of product development," Harv. Bus. Rev., vol. 90, no. 5, pp. 84-94, 2012. 
[259] Y. T. Chong and C. H. Chen, "Management and forecast of dynamic customer needs: An artificial immune and neural system approach," Adv. Eng. Informatics, vol. 24, no. 1, pp. 96-106, 2010.

[260] E. Heiskanen, K. Hyvönen, M. Niva, M. Pantzar, P. Timonen, and J. Varjonen, "User involvement in radical innovation: are consumers conservative?," Eur. J. Innov. Manag., vol. 10, no. 4, pp. 489-509, 2007.

[261] K. Siau and X. Tan, "Improving the quality of conceptual modeling using cognitive mapping techniques," Data Knowl. Eng., vol. 55, no. 3, pp. 343-365, 2005.

[262] G. Coyle and D. Exelby, "The validation of commercial system dynamics models," Syst. Dyn. Rev., vol. 16, no. 1, pp. 27-41, 2000.

[263] P. M. Senge and J. W. Forrester, "Tests for building confidence in system dynamics models," Syst. Dyn. TIMS Stud. Manag. Sci., vol. 14, pp. 209-228, 1980.

[264] L. F. Luna-Reyes and D. L. Andersen, "Collecting and analyzing qualitative data for system dynamics: Methods and models," Syst. Dyn. Rev., vol. 19, no. 4, pp. 271-296, 2003.

[265] S. J. Tracy, 'Qualitative quality: Eight 'big-tent' criteria for excellent qualitative research," Qual. Inq., vol. 16, no. 10, pp. 837-851, 2010.

[266] C. J. Atman et al., "Enabling engineering student success: the final report for the center for the advancement of engineering education. CAEE-TR-10-02," Cent. Adv. Eng. Educ., p. 224, 2010.

[267] T. A. Litzinger, L. R. Lattuca, R. G. Hadgraft, and W. C. Newstetter, "Engineering education and the development of expertise," J. Eng. Educ., vol. 100, no. 1, pp. 123-150, 2011.

[268] K. Brundiers, A. Wiek, and C. L. Redman, "Real-world learning opportunities in sustainability: from classroom into the real world," Int. J. Sustain. High. Educ., vol. 11, no. 4, pp. 308-324, 2010.

[269] C. L. Cobb, A. M. Agogino, and S. Kim, “Advances in engineering education what alumni value from new product development education : a longitudinal study what alumni value from new product development education," Adv. Eng. Educ., vol. 5, no. 1, pp. 1-37, 2016.

[270] B. Yoon and A. J. Jetter, "Comparative analysis for Fuzzy Cognitive Mapping," in 2016 Portland International Conference on Management of Engineering and Technology (PICMET), 2016, pp. 1897-1908.

[271] R. A. Krueger and M. A. Casey, Focus Groups - A Practical Guide for Applied Research, 5th ed. Thousand Oaks, CA: SAGE Publications, Inc, 2015.

[272] N. Eidmohammadi, "Wet and dry robotic vacuum cleaner," M.S. Thesis, Dept. Prod. Prod. Dev., Chalmers Univ. of Tech., Gothenburg, Sweden ,2014.

[273] R. Mateo Ferrús and M. Domínguez Somonte, "Design in robotics based in the voice of the customer of household robots," Rob. Auton. Syst., vol. 79, pp. 99-107, 2016.

[274] J. Montalván, H. Shin, F. Cuéllar, and K. Lee, “Adaptation profiles in first-time robot users: Towards understanding adaptation patterns and their implications for design," Int. J. Des., vol. 11, no. 1, pp. 1-19, 2017. 
[275] J.-Y. Sung, "Towards the human-centered design of everyday human robots," Ph.D. Dissertation, College of Comput. Georgia Inst. of Technol., Atlanta, GA 2011.

[276] Z. Dikopoulou and E. Papageorgiou, "fcm: inference of fuzzy cognitive maps (FCMs)." 2017.

[277] W. J. Tastle and M. J. Wierman, "Consensus and dissention : a measure of ordinal dispersion," Int. J. Approx. Reason., vol. 45, no. 3, pp. 531-545, 2007.

[278] W. J. Tastle, M. J. Wierman, and U. R. Dumdum, "Ranking ordinal scales using the consensus measure," Issues Inf. Syst., vol. 6, no. 2, pp. 96-102, 2005.

[279] K. Langfield-Smith and A. Wirth, "Measuring differences between cognitive maps," J. Oper. Res. Soc., vol. 43, no. 12, p. 1135, Dec. 1992.

[280] G. Csardi and T. Nepusz, "The igraph software package for complex network research," InterJournal, vol. Complex Sy, p. 1695, 2006.

[281] A. B.V., B. Thieurmel, and T. Robert, "visNetwork: network visualization using "vis.js' library." 2019.

[282] D. M. Hamby, "A review of techniques for parameter sensitivity analysis of environmental models," Environ. Monit. Assess., vol. 32, no. 2, pp. 135-154, Sep. 1994.

[283] S. Rhim, J.-C. Ryu, K.-H. Park, and S.-G. Lee, "Performance evaluation criteria for autonomous cleaning robots," in 2007 International Symposium on Computational Intelligence in Robotics and Automation, 2007, pp. 167-172.

[284] G. M. Sullivan and A. R. Artino, "Analyzing and interpreting data from likert-type scales," J. Grad. Med. Educ., vol. 5, no. 4, pp. 541-542, 2014.

[285] S. E. Harpe, "How to analyze Likert and other rating scale data," Curr. Pharm. Teach. Learn., vol. 7, no. 6, pp. 836-850, 2015.

[286] M. Amer, A. Jetter, and T. Daim, "Development of fuzzy cognitive map (FCM) based scenarios for wind energy," Int. J. Energy Sect. Manag., vol. 5, no. 4, pp. 564-584, Nov. 2011.

[287] I. N. C. Bang, G. Heo, Y. H. Jeong, and S. U. N. Heo, "An axiomatic design approach of nanofluid- engineered nuclear safety features for generation III + reactors," Nucl. Eng. Technol., vol. 41, no. 9, pp. 1157-1170, 2009.

[288] N. P. Suh, "Axiomatic design theory for systems," Res. Eng. Des., vol. 10, no. 4, pp. 189-209, 1998.

[289] Davdas Shetty, Product Design for Engineers, 1st ed. Boston, MA: Cengage Learning, 2016.

[290] N. P. Suh, "Ergonomics, axiomatic design and complexity theory," Theor. Issues Ergon. Sci., vol. 8, no. 2, pp. 101-121, 2007.

[291] C. E. Shannon, The Mathematical Theory of Communication. Champaign, IL: University of Illinois press, 1998.

[292] M. Nagamachi, "Kansei Engineering: a new ergonomic consumer-oriented technology for product development," Int. J. Ind. Ergon., vol. 15, no. 1, pp. 3-11, 1995. 


\section{Appendix A - Axiomatic Design Approach (ADA)}

ADA provides NPD teams with systemic principles for seeking a best design fulfilling customer requirements with minimum complexity[200]-[202], [287]. Particularly, functional requirements (FRs) are developed from these drawn product design factors. In this matter, each FR should be independent from other FRs (Axiom 1: Independence Axiom). NPD teams can bring corresponding engineering and technological alternatives as design parameters (DPs) for the defined FRs, wherein each DP should include minimum information content (Axiom 2: Information Axiom). These two fundamental axioms derive following design rules as corollaries [288], [289]:

- Corollary 1 - decoupling of coupled design

Decouple or separate parts or aspects of a solution if FRs are coupled or become interdependent in the designs proposed.

- Corollary 2 - minimization of FRs

Minimize the number of FRs and constraints.

- Corollary 3 - integration of physical parts

Integrate design features in a single physical part if FRs can be independently satisfied with the proposed solution.

- Corollary 4 - use of standardized and interchangeable parts

Use standardized or interchangeable parts if they are consistent with the FRs and constraints.

- Corollary 5 - use of symmetry

Use symmetrical shapes and/or arrangements if they are consistent with FRs and constraints.

- Corollary 6 - largest tolerance

Specify the largest allowable tolerance in stating FRs.

- Corollary 7 - uncoupled design with less information

Seek an uncoupled design that requires less information than coupled designs in satisfying a set of FRs. 
Based on these corollaries, NPD teams keep on decomposing FRs \& DPs to subordinate FRs \& DPs until the first axiom is satisfied. As a result, ADA yields the design hierarchy linking high-level FRs \& DPs (conceptual design) and low-level features or parameters by top-down decomposition [287]. The figure below depicts an example of a design hierarchy.

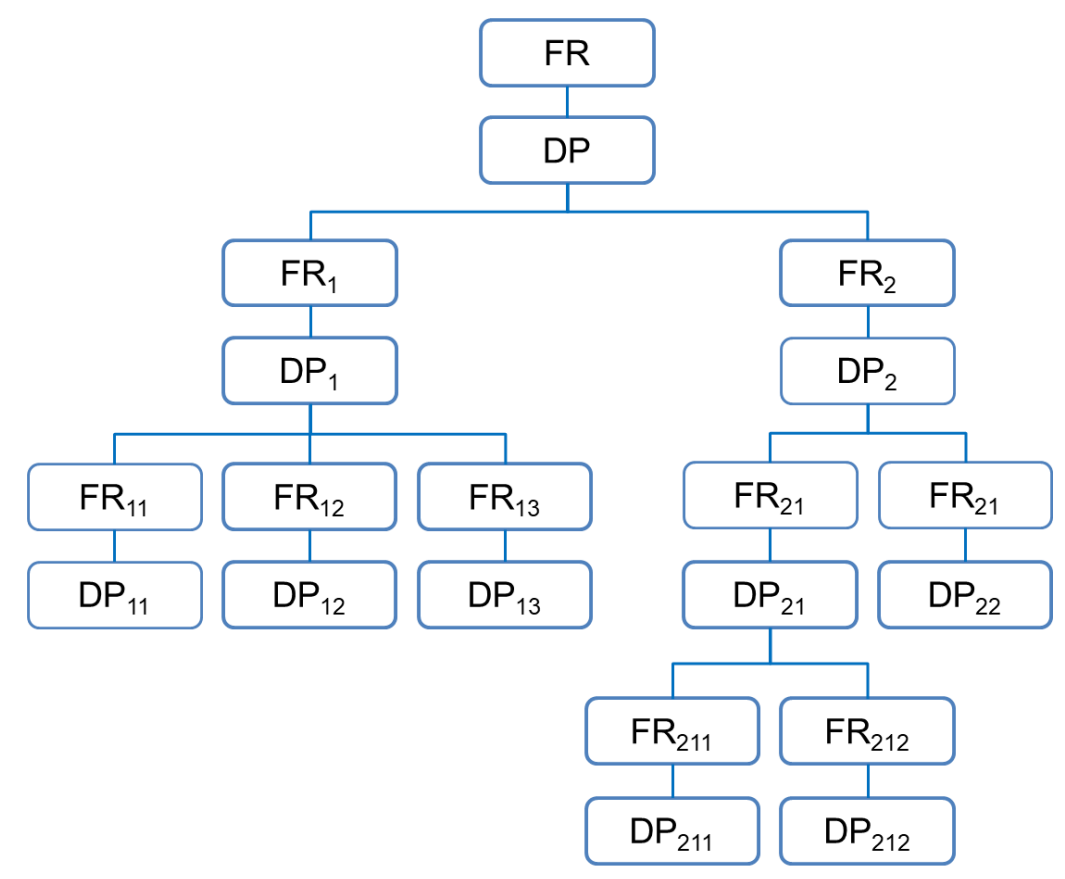

A design hierarchy mapping FRs and DPs

The mathematical representation of the first axiom can be described as follows:

$$
[F R]=[D M][D P]
$$

where

$[F R]=$ vector of the functional requirements

$[D P]=$ vector of design parameters (physical parameters, parts, or subassemblies)

$[D M]=$ relationship matrix between functional and physical domain 
In achieving the independent FRs (Axiom 1), DM should be diagonal or triangular, which means uncoupled or decoupled designs correspondingly, whereas designs are coupled.

Furthermore, the Information Axiom (Axiom 2) is a fundamental measure in selecting the optimized and robust design among multiple design concepts based on information theory [206], [290], [291]. According to Suh [201], [290], information content $I_{i}$ for a given $F R_{i}$ is defined as:

$$
I_{i}=\log _{2}\left(\frac{1}{p_{i}}\right)=-\log _{2} p_{i}
$$

where $p_{i}$ is the probability of fulfilling $F R_{i}$. Because a target product is regarded as a combination of several FRs, the information content of a product is the sum of information contents corresponding FRs as follows:

$$
I_{\text {prt }}=\log _{2}\left(\frac{1}{p_{\{m\}}}\right)=-\log _{2} p_{\{m\}}=-\log _{2} \prod_{i=1}^{m} p_{i}=-\sum_{i=1}^{m} \log _{2} p_{i}
$$

where $m$ is the number of FRs. From the definition of the information content, if the probability is close to 1.0 , the information content is converged to zero. Conversely, the information content is diverged to infinity when the probability is zero. Therefore, the best product concept is to have the minimum information content. 


\section{Appendix B - Product Design Requirement Document}

\section{Product Requirements Document for a New Home Floor Cleaning Robot, UniCleanBot}

Version: 2 (11.13.2018)

Written by: Byung Sung Yoon

Reviewed by:

\section{Introduction}

\section{Purpose of Document}

This document aims to provide the product requirements for a robotic device, socalled UniCleanBot, to serve as wet and dry cleaning of rigid or carpet-covered floors autonomously in typical home, as a target product for an experiment.

\section{Scope}

This document focuses on providing the product requirements of a product for actual development.

The described market research information, customer statements and interpreted needs in this document are mainly extracted from a master thesis, written by Eidmohammadi (2014), studying a conceptual solution for automated vacuuming and mopping of house floors. Moreover, it includes some contents from other research papers studying customer needs on home robotic cleaning systems during last five years.

\section{Market Problem}

\section{Consumer Problem}

As the households of today are becoming smarter and more automated, people have a positive attitude toward adopting automated solutions making their life more 
convenient and comfortable. In this regard, customers want a smart and automated solution for floor cleaning as one of household chores recognized as time-consuming and repetitive work.

Customers expect a smart and automated solution enabling to clean various conditions of home floors that are made of hard materials (e.g. marble, wood and tile) or covered with carpet. Moreover, they want to minimize additional interventions (e.g., emptying the dustbin, moving a device to a specific location, adding some cleaning materials into a device, and so on) in the cleaning cycle.

Customers also expect intuitive, easy, universal interactions with a device in installing, setting up, using and maintaining. For example, customers sometimes want to control or monitor remotely smart appliances located at home with their handheld smart devices.

Customers are concerned with noise and allergens spreading through the air during traditional cleaning work.

\section{Product Problem}

Currently, there are few commercial products (home floor cleaning robots, HFCRs) equipped with multiple cleaning methods (e.g. vacuuming, mopping, sweeping, or scrubbing) to cover various floor conditions in a single device. If at all, their performance is ineffective overall.

The price range of such commercial products varies (between $\$ 20$ and $\$ 1,000$ ) according to which functions they provide, and are relatively more expensive than traditional vacuums.

\section{Technology Problem}

In practical usage environments of HFCRs, there are various physical obstacles interrupting their cleaning work (e.g., furniture, stairs, moving objects, and household 
items). Especially, the height and the diameter of HFCRs are limited in width for the accessibility under furniture, such as beds, couches, and chairs. Moreover, the shape of HFCRs is also crucial to cleaning the corners of a room. The picture below depicts the size limitation and various shapes of HFCRs. These constraints cause hardship in designing and arranging their main components such as dustbin, water tank, battery, suction unit, mopping (or sweeping) pad or motor unit.
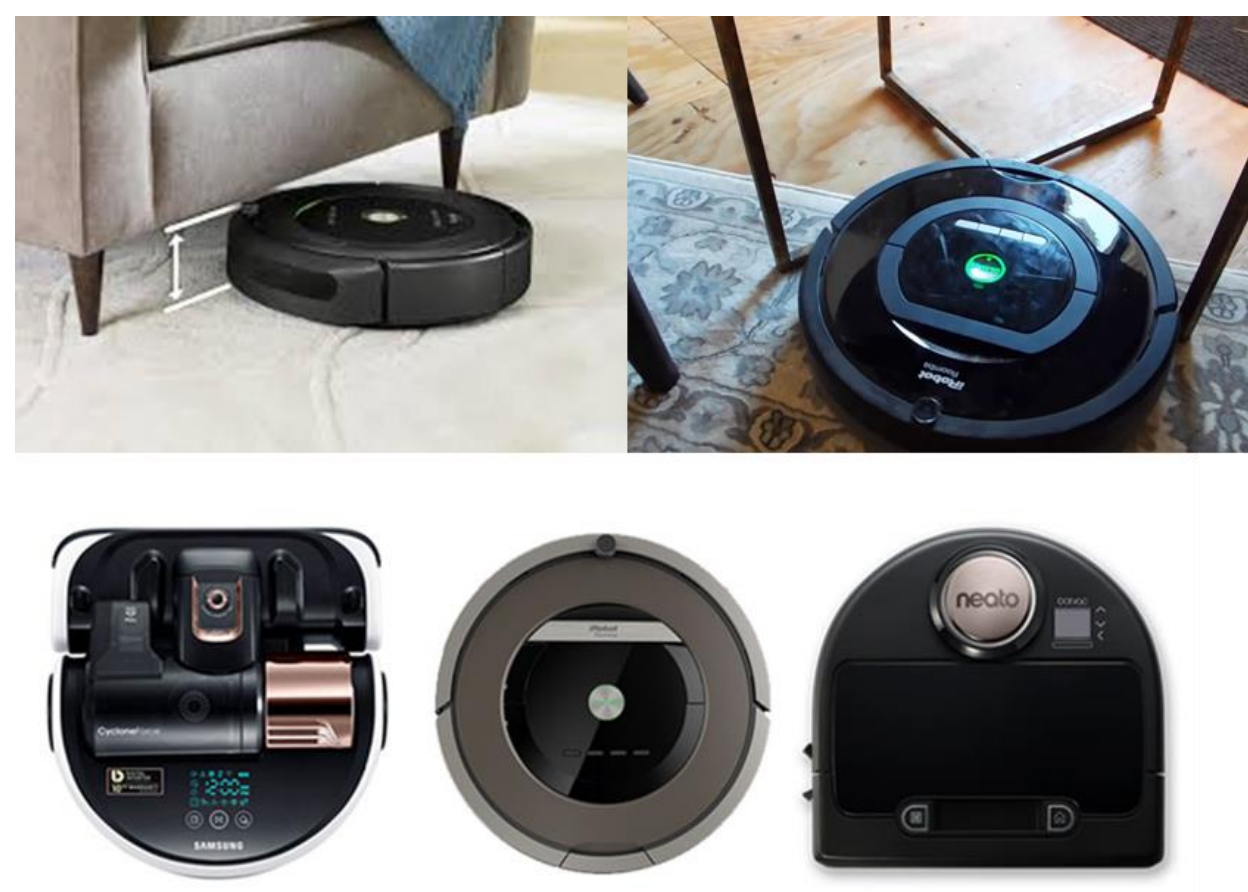

\section{HFCRs have limitations of size and shape.}

Because the working environment is not fixed but changes frequently, an HFCR has to detect location information and map the area around it from time to time. Therefore, it is important to develop and select proper sensors and a specific pathplanning algorithm for increasing the cleaning efficiency of an HFCR. However, it is necessary to consider trade-offs associated with the power management strategy as well as limited size of an HFCR. The table below shows examples of sensors and path-planning algorithm for HFCRs. 
Examples of sensors and path-planning algorithm for HFRCs

\begin{tabular}{|l|l|}
\hline $\begin{array}{l}\text { Sensors for Location and Obstacle } \\
\text { Detection }\end{array}$ & Path-planning Algorithm \\
\hline Indoor GPS System & Random Walk Based Algorithm \\
Ultrasonic or Infrared (IR) Sensors & Spiral Algorithm \\
Wide-angle Vision Camera & Snaking Algorithm \\
Simultaneous Localization and Mapping & Wall Follow Algorithm \\
(SLAM) & Path Transform \\
Bumper Sensors & Genetic Algorithm \\
\hline
\end{tabular}

In the usage environment, an HFCR encounters various types of surfaces. When passing over from hard floor to thick or deep carpet, an HFCR may become stuck. To prevent this and to ensure its mobility, it is essential to include a special driving unit. Furthermore, as the occasion demands, it is necessary to set up custom cleaning boundaries with cliff detection sensors or boundary markers (e.g., Virtual Wall ${ }^{\circledR}$ or magnetic strip) for an HFCR to control where it can clean.

An HFCR is powered by a battery unit during its cleaning cycle except when being charged. During the cleaning cycle, most of the functional units in an HFCR consume electric power supplied by the battery unit continuously. Particularly, the energy capacity of the battery unit is spent considerably by the multiple motor units for suction and driving. Therefore, ensuring enough cleaning time is impossible without including a high capacity or high efficient battery unit as well as a power management system, including charging the battery autonomously.

\section{General Description of Product}

\section{Product Description}

A robotic device to serve as wet and dry-cleaning of rigid or carpet-covered floors autonomously in typical home.

\section{Product Functions and Features}


The following functions and features should be incorporated into the proposed device design.

<For Competent Cleaning Performance $>$

...allows dry and wet cleaning for hard floor.

...allows dry cleaning for carpet-covered floor.

...allows dry and wet cleaning along edges as close as possible.

...allows dry and wet cleaning of corners as deep as possible.

...is sealed enough not to drop dust and water.

...dries the floor sufficient after wet cleaning.

...is capable of autonomously detecting whether the working surface is hard or carpet-covered floor.

...is capable of detecting amount of dust or dirt in different spots from a distance.

...recognizes dirtier areas and cleans them more extensively.

...removes allergens and bacteria.

...removes pet hair

$<\underline{\text { For Operational Excellence }>}$

...remembers the cleaning path from previously cleaned rooms.

...updates the cleaning path whenever there are any changes in rooms.

...allows its operation in rooms absent of light.

...is capable of climbing thresholds and carpets. 
...is free from pre-treatment of floor.

...has a plenty of dustbin and water tank capacity.

...has washable dustbin.

...is usable with any type of detergent.

...prevents tangling of hair or strings on parts.

...is capable to resume where it was cleaning automatically, if cleaning cycle was not finished or was interrupted.

...allows scheduled wet $\&$ dry cleaning.

...allows scheduled charging.

...has an intuitive user interface.

...is capable of recognizing speech.

...has a comfortable grip for moving and handling.

...has a durable lifetime.

...informs the user when in need of emptying.

...informs the user when in need of water refill.

...informs the user when in need of maintenance.

...allows the user to control and monitor it through a wireless network.

$<$ For Intelligent Power Management $>$

...has a plenty of battery capacity enabling dry cleaning for a separate family room ( $50 \mathrm{~m} 2$ or $540 \mathrm{ft} 2$ at least) that has a carpeted floor in one charge. 
...has a plenty of battery capacity enabling wet- \& dry-cleaning for a hard floor area (20 $\mathrm{m} 2$ or $215 \mathrm{ft} 2$ at least) in one charge.

...has fast charging time.

...has charging stations enabling charging its battery automatically whenever needed.

...is capable of recognizing the location of the charging stations autonomously.

...has charging stations occupying a small space.

<For Easy Maintenance $>$

...allows easy replacement of worn parts.

...is easy to assemble and disassemble.

...is easy to empty dustbin and dirty water.

...is easy to refill clean water and detergent.

...is easy to remove tangled hair or strings from parts.

...is easy to set up for start.

...is easy to update firmware.

<For Careful Safety>

...recognizes overhead barriers and avoids them.

...is capable of detecting obstacles before bumping into them.

...recognizes if it is stuck and stops cleaning.

...recognizes if operating on a carpet and immediately stops wet cleaning.

...recognizes when cables, wires or curtains were tangled, and detangles them. 
...recognizes if it suctions metal and informs the user.

...has the electronics isolated from water.

...prevents leakage.

...prevents affecting the floor after a cleaning cycle when in standby.

...prevents leaving marks on furniture or walls during and after cleaning.

...prevents scratching the floor.

\section{User Characteristics}

US household having median household income

People between 19-40 years old being familiar with technologies

Family with children and pet-owners

People living in a typical home with hard and carpet-covered floor.

\section{General Constraints}

The device must not have a shape easily stuck under furniture.

The device must have a shape easily accessible to corners of a room.

The device must be light enough to be moved with human hands.

The device cannot move between floor levels.

\section{Assumptions and Dependencies}

This device is used within indoor environment only.

This device is operated under normal conditions (NTP - Normal Temperature and Pressure). 


\section{External Interface Requirement}

Standardized power outlets supplying electricity to the charging station of the device (120volts at a frequency of $60 \mathrm{~Hz}$ in US).

Wireless communication environment allowing customers to control and monitor the device remotely (e.g. Wifi or Mobile Network) using a specific app. 


\section{Appendix C - User Personas}

User Personas for a NEW Home Floor Cleaning Robot

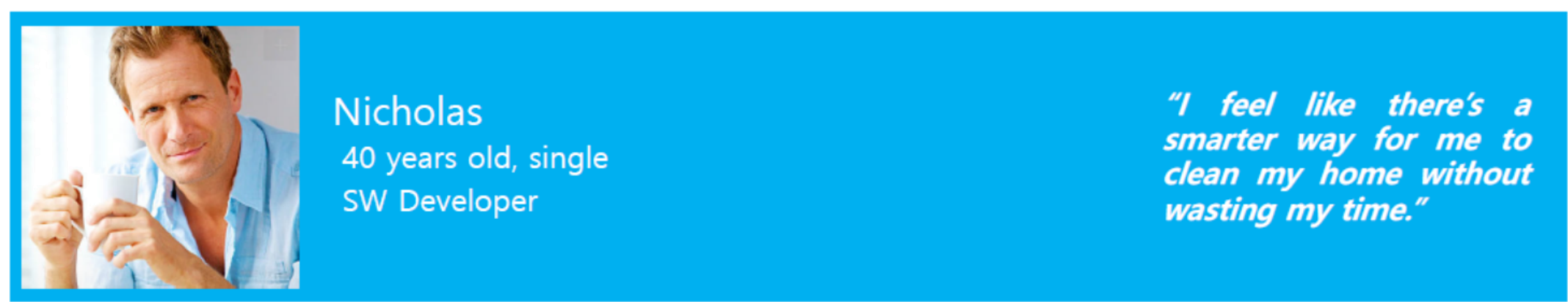

Nicholas is a busy professional who specializes in SW development. He is very tech savvy and enjoys trying a new thing. He lives in an apartment in the periphery of town.

\section{Floor Cleaning Tool Owner}

A handheld vacuum cleaner and a spray floor mop

\section{Price Sensitivity}

Willing to pay for a new gadget to reduce his effort of daily chores

\section{Frequency}

Vacuums once per month and rarely uses a spray stick mop for his hard floor

\section{Behavior}

- Cleaning is not a big matter

- Stores cleaning equipment in the closet

- Spends up to one hour for cleaning

- Spends most of his time outside

\section{Pain Points}

-Using multiple tools for floor cleaning

- No time for cleaning

\section{Needs}

-Wants to spend less time for cleaning

-Wants to minimize cleaning effort

-Wants to keep home clean

\section{Features}

- Scheduled wet \& dry floor cleaning

-Automatic charging and emptying the dustbin 
User Personas for a NEW Home Floor Cleaning Robot

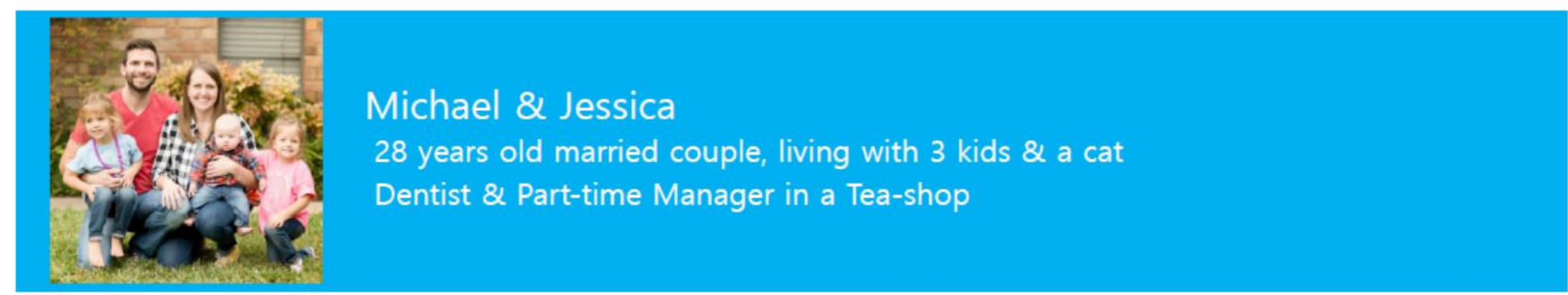

The family consists of the parents (late 20s), two daughters ( $5 \& 4$ years old), a 6 months old baby boy and a cat. They live in the countryside, and use new technology as long as it is practical.

\section{Floor Cleaning Tool Owner}

A canister and a handheld vacuum cleaners, an electric mop/hard floor cleaner, and a steam cleaner

Price Sensitivity

Willing to pay for a floor cleaning tool

Frequency

Cleaning on a daily basis

\section{Behavior}

-Maintaining an organic lifestyle

- Noticing every little piece of dirt

- Constantly feeling some pressure to clean or tidy up

- Vacuuming and mopping more or less on a daily basis

\section{Pain Points}

-Allergens and bacteria spreading through the air

- Pet hair from the cat

-Dust and dirt from the garden

- Tangled hair on the brush of the vacuum cleaner

\section{Needs}

-Want to use tools instantly without effort to setting up

-Want to control and monitor a cleaning tool remotely

-Want to keep home clean for kids" health

\section{Features}

- Scheduled wet \& dry floor cleaning

- Including HEPA filter for filtering allergens and bacteria in the outlet air

-Wireless network connection 


\section{Appendix D - Surveys for Experiment}

\section{Survey 1}

\section{P Portland State}

Introduction

This is the first survey to ask your understanding of the product system in UniCleanBot prior to the product design workshop scheduled on Feb. 23rd.

If you are ready, please go to the next page. 


\section{Portland State}

\section{Product Background}

To prepare the upcoming workshop we need to tell you about the target product. It is a cleaning robot for floors in private homes. It can vacuum, sweep, or wash the floor. We call it the UniCleanBot.

It is important that you familiarize your self with this product because we will ask you questions about it. Please watch the video below.

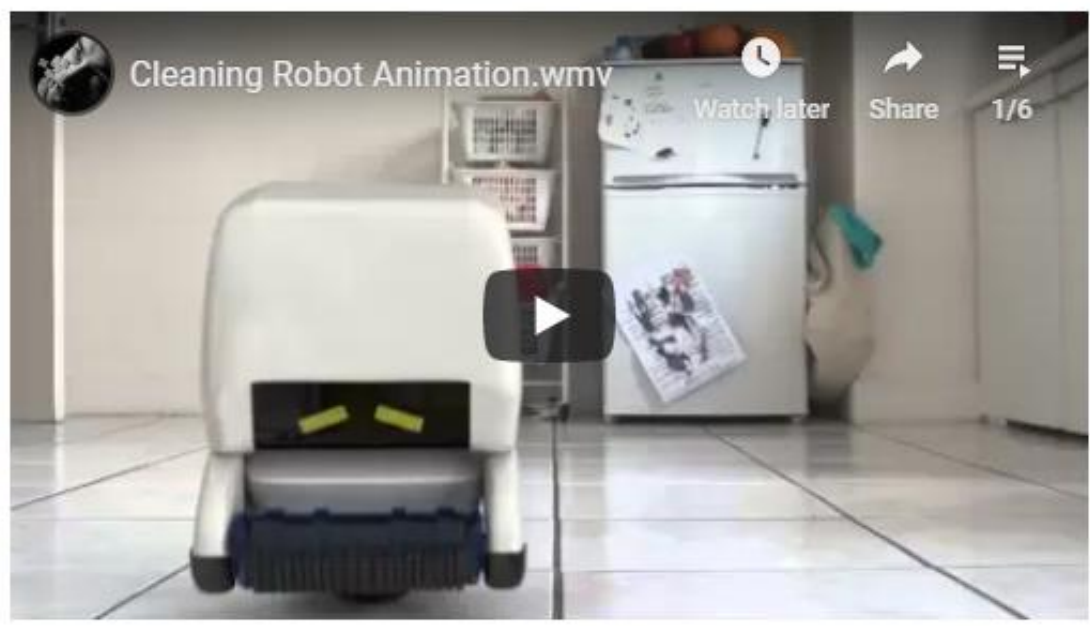

Please confirm that you have watched the video clip.

O Yes, I have watched the video.

O No, I have not watched the video. 


\section{\& Portland $\underset{\text { UNIVERSITY }}{\text { State }}$}

\section{Product Requirements}

Before starting to develop a new product, development teams receive a document with so-called product design requirements. The document clarifies expectations for the future product. Below are the requirements for the UniCleanBot. Please read the document. (You are not expected to remember all the details but you should read the entire document).

Product Design Requirements Document

Please confirm that you have read the product design requirements document.

Yes, I have read the document.

No, I have not read the document.

\section{$\int$ Portland $\int_{\text {S IVE RSITY }}$}

\section{User Persona}

Development teams also need to know about the user(s) of the future product. This is done with a user persona. Below is a user persona for the UniCleanBot. Please read it. (You are not expected to remember all the details but you should read the entire document).

\section{User persona for UniCleanBot}

Please confirm that you have read the document.

Yes, I have read the user persona.

No, I have not read the user persona. 


\section{Portland State}

Thank you for taking the time to learn about the UniCleanBot. We will ask you a few questions about this product.

\section{\& Portland State}

\section{Questions about product features and performance}

Product features can impact how customers perceive the overall performance of the product. For example, if a product looks sleek and modern, they may think that it has sophisticated technology.

We will ask you about your opinion on how product features impact how customers perceive important aspects of the performance of the UniCleanBot, such as cleaning power or safety. 


\section{@ Portland State}

\section{Question \#1-1}

The team is considering an Energy Level Indicator for the Battery which shows the remaining battery capacity of UniCleanbot. When the Energy Level Indicator is implemented, how do the customers think that the performance factors below change?

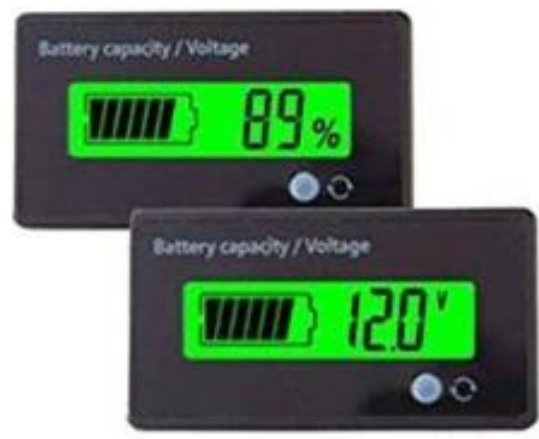

\begin{tabular}{|c|c|c|c|c|c|c|c|}
\hline \multicolumn{5}{|c|}{$\begin{array}{l}\text { Example } \\
\text { If you judge that the customers think that Operational Excellence will increase } \\
\text { moderately when the Energy Level Indicator is implemented, you need to } \\
\text { select the corresponding point, "increase moderately." }\end{array}$} & \multirow[b]{2}{*}{$\begin{array}{l}\text { increase } \\
\text { slightly }\end{array}$} & \multirow[b]{2}{*}{$\begin{array}{l}\text { increase } \\
\text { moderately }\end{array}$} & \multirow[b]{2}{*}{$\begin{array}{l}\text { increase } \\
\text { extremel }\end{array}$} \\
\hline & $\begin{array}{l}\text { decrease } \\
\text { extremely }\end{array}$ & $\begin{array}{l}\text { decrease } \\
\text { moderately }\end{array}$ & $\begin{array}{l}\text { decrease } \\
\text { slightly }\end{array}$ & $\begin{array}{l}\text { remain the } \\
\text { same }\end{array}$ & & & \\
\hline Cleaning Performance & 0 & 0 & 0 & 0 & 0 & 0 & 0 \\
\hline Operational Excellence & 0 & 0 & 0 & 0 & 0 & 0 & 0 \\
\hline $\begin{array}{l}\text { Intelligence of Power } \\
\text { Management }\end{array}$ & 0 & 0 & 0 & 0 & 0 & 0 & 0 \\
\hline Ease of Maintenance & 0 & 0 & 0 & 0 & 0 & 0 & 0 \\
\hline Safety-in-Use & 0 & 0 & 0 & 0 & 0 & 0 & 0 \\
\hline
\end{tabular}




\section{P Portland State}

\section{Question \#1-2}

The team is considering a Water Proof Design for the product. When the Water Proof Design is implemented, how do the customers think that the performance factors below change?

\section{Example}

If you judge that customers would think that operational excellence will increase slightly when the Water Proof Design is implemented, you need to select the corresponding point, "increase slightly."

$\begin{array}{lccccccc} & \begin{array}{c}\text { decrease } \\ \text { extremely }\end{array} & \begin{array}{c}\text { decrease } \\ \text { moderately }\end{array} & \begin{array}{c}\text { decrease } \\ \text { slightly }\end{array} & \begin{array}{c}\text { remain the } \\ \text { same }\end{array} & \begin{array}{c}\text { increase } \\ \text { slightly }\end{array} & \begin{array}{c}\text { increase } \\ \text { moderately }\end{array} & \begin{array}{c}\text { increase } \\ \text { extemely }\end{array} \\ \begin{array}{l}\text { Cleaning Performance } \\ \text { Operational Excellence }\end{array} & 0 & 0 & 0 & 0 & 0 & 0 & 0 \\ \begin{array}{l}\text { Intelligence of Power } \\ \text { Management }\end{array} & 0 & 0 & 0 & 0 & 0 & 0 & 0 \\ \begin{array}{l}\text { Ease of Maintenance } \\ \text { Safety-in-Use }\end{array} & 0 & 0 & 0 & 0 & 0 & 0 & 0\end{array}$




\section{P Portland State}

\section{Question \#1-3}

The team is considering a Face Recognition that means the ability to automatically recognize human faces based on dynamic facial images. When the Face Recognition is implemented, how do the customers think that the performance factors below change?

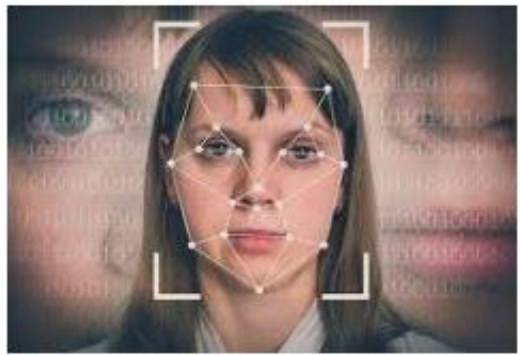

$\begin{array}{lccccccc}\text { Cleaning Performance } & \begin{array}{l}\text { decrease } \\ \text { extremely }\end{array} & \begin{array}{c}\text { decrease } \\ \text { moderately }\end{array} & \begin{array}{c}\text { decrease } \\ \text { slightly }\end{array} & \begin{array}{c}\text { remain the } \\ \text { same }\end{array} & \begin{array}{c}\text { increase } \\ \text { slightly }\end{array} & \begin{array}{c}\text { increase } \\ \text { moderately }\end{array} & \begin{array}{c}\text { increase } \\ \text { extremely }\end{array} \\ \begin{array}{l}\text { Operational Excellence } \\ \begin{array}{l}\text { Intelligence of Power } \\ \text { Management }\end{array}\end{array} & 0 & 0 & 0 & 0 & 0 & 0 & 0 \\ \begin{array}{l}\text { Ease of Maintenance } \\ \text { Safety-in-Use }\end{array} & 0 & 0 & 0 & 0 & 0 & 0 & 0 \\ \text { O } & 0 & 0 & 0 & 0 & 0 & 0 & 0\end{array}$




\section{P Portland State}

\section{Question \#1-4}

The team is considering a Step-climbing Mechanism enabling UniCleanBot to climb stairs and move from floor to floor. When the Step-climbing Mechanism is implemented, how do the customers think that the performance factors below change?

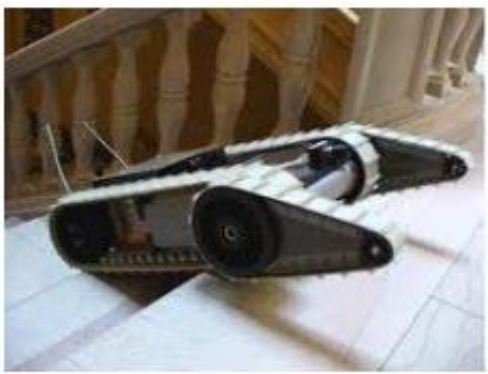

$\begin{array}{lccccccc}\text { decrease } & \begin{array}{c}\text { decrease } \\ \text { modertemely }\end{array} & \begin{array}{c}\text { decrease } \\ \text { slightly }\end{array} & \begin{array}{c}\text { remain the } \\ \text { same }\end{array} & \begin{array}{c}\text { increase } \\ \text { slightely }\end{array} & \begin{array}{c}\text { increase } \\ \text { moderately }\end{array} & \begin{array}{c}\text { increase } \\ \text { extremely }\end{array} \\ \text { Cleaning Performance } & 0 & 0 & 0 & 0 & 0 & 0 & 0 \\ \begin{array}{l}\text { Operational Excellence } \\ \begin{array}{l}\text { Intelligence of Power } \\ \text { Management }\end{array}\end{array} & 0 & 0 & 0 & 0 & 0 & 0 & 0 \\ \begin{array}{l}\text { Ease of Maintenance } \\ \text { Safety-in-Use }\end{array} & 0 & 0 & 0 & 0 & 0 & 0 & 0\end{array}$




\section{赵 Portland State}

\section{Question \#1-5}

The team is considering a Self-emptying Dustbin which enable the UniCleanBot to empty its dustbin automatically at the charging station. When the Self-emptying Dustbin is implemented, how do the customers think that the performance factors below change?

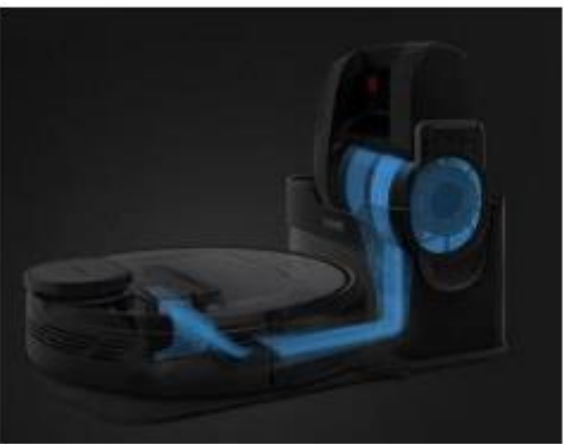

decrease decrease decrease remain the increase increase increase
extremely moderately slightly same slightly moderately extremely

Cleaning Performance

Operational Excellence

Intelligence of Power

Management

Ease of Maintenance

Safety-in-Use
0

O

o

0

0
0

o

0

0

0

0

o

0

0

0

0

0

o

0

0

0

o

0

0

0

0

0

0

0

0

0

0

o 


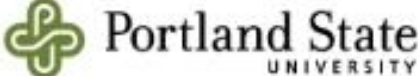

\section{Question \#1-6}

The team is considering a HEPA Filter (High Efficiency Particulate Air Filter) that filters out small dust particals. The air that leaves the vacuum is thus cleaner than the air it sucks in. When the HEPA filter is implemented, how do the customers think that the performance factors below change?

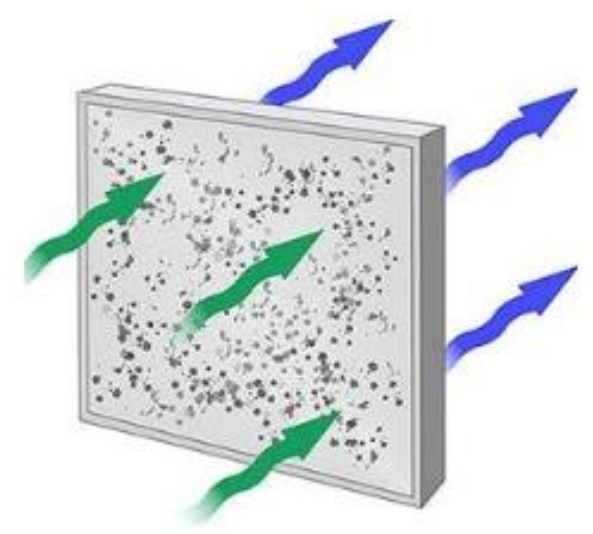

Cleaning Performance

Operational Excellence

$$
\begin{aligned}
& \text { decrease } \\
& \text { extremely }
\end{aligned}
$$

$$
\begin{gathered}
\text { decrease } \\
\text { moderately }
\end{gathered}
$$

decrease

remain the

increase same

slightly

increase

moderately

increase

o

$\circ$

$\circ$

0

0

$\circ$

extremely

$\circ$

$\circ$

$\circ$

$\circ$

$\circ$

$\circ$

$\circ$

Intelligence of Power

Management

Ease of Maintenance

Safety-in-Use

$\begin{array}{lll}0 & 0 & 0 \\ 0 & 0 & 0 \\ 0 & 0 & 0\end{array}$

o

0

o

o

o

0

o

○

○

o

o

o 


\section{蛋 Portland State}

\section{Question \#1-7}

The team is considering an Expandable Corner Brush which enables the UniCleanBot to clean dust in comers. When the Expandable Corner Brush is implemented, how do the customers think that the performance factors below change?

\begin{tabular}{|c|c|c|c|c|c|c|c|}
\hline & $\begin{array}{l}\text { decrease } \\
\text { extremely }\end{array}$ & $\begin{array}{l}\text { decrease } \\
\text { moderately }\end{array}$ & $\begin{array}{l}\text { decrease } \\
\text { slightly }\end{array}$ & $\begin{array}{l}\text { remain the } \\
\text { same }\end{array}$ & $\begin{array}{l}\text { increase } \\
\text { slightly }\end{array}$ & $\begin{array}{l}\text { increase } \\
\text { moderately }\end{array}$ & $\begin{array}{l}\text { increase } \\
\text { extremely }\end{array}$ \\
\hline Cleaning Performance & 0 & 0 & 0 & 0 & 0 & 0 & 0 \\
\hline Operational Excellence & 0 & 0 & 0 & 0 & 0 & 0 & 0 \\
\hline $\begin{array}{l}\text { Intelligence of Power } \\
\text { Management }\end{array}$ & 0 & 0 & 0 & 0 & 0 & 0 & 0 \\
\hline Ease of Maintenance & 0 & 0 & 0 & 0 & 0 & 0 & 0 \\
\hline Safety-in-Use & 0 & 0 & 0 & 0 & 0 & 0 & 0 \\
\hline
\end{tabular}

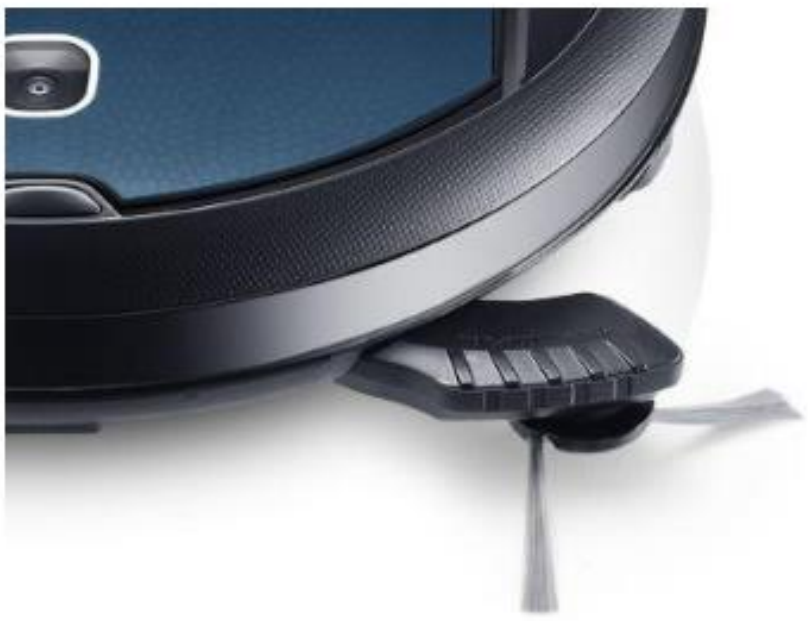




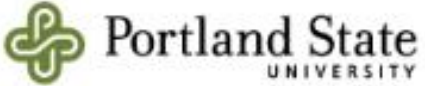

\section{Question \#1-8}

The team is considering a Lower Profile Design which enables the UniCleanBot to access space under the couch, bed, or any type of furniture in the house. When the Lower Profile design is implemented, how do the customers think that the performance factors below change?

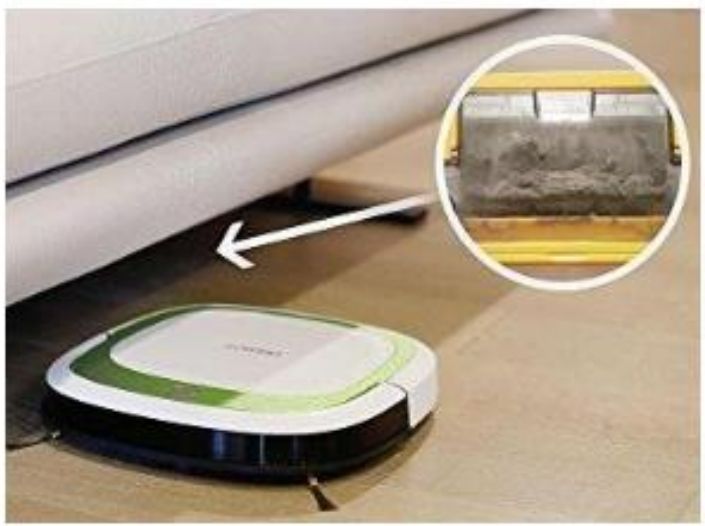

$$
\begin{array}{ccccc}
\text { decrease decrease decrease remain the increase } & \text { increase increase } \\
\text { extremely moderately slightly } & \text { same } & \text { slightly } & \text { moderately extremely }
\end{array}
$$

Cleaning Performance

Operational Excellence

Intelligence of Power

Management

Ease of Maintenance

Safety-in-Use
O

O

O

O

0

0

0

0

0

0

o

0

o

o

0

o

0

0

0

0

0

0

0

0

O

O

O

0

0

0

0 


\section{P Portland State}

\section{Questions about customer preference and adding features}

Below is a list of Product Design Factors (or features) that the product development team could implement: In which way would implementing these features impact customer preference (i.e. how much customers like the product)?

Example

If you judge that customers will like UniCleanBot moderately better by implementing the Energy Level Indicator, you need to place the slider on the corresponding level, Moderately better. Battery

Water Proof Design

Face Recognition

Step-climbing Mechanism

Self-emptying Dustbin

HEPA Filter

Expandable Corner Brush

Low Profile Design

Much Moderately Slightly

worse worse worse

About the

same

Slightly

better

Moderately

better

Much

better

○

○

○

○

○

○

○

$\circ \quad 0$

○

$\circ$

$\circ$

○

○

o

○

$\circ$

$\circ$

0

0

$\circ$

$\circ \quad 0$

○

o

○

○

○

o

o

o

○

o

o

○

○

○

o

○

o

o

o

o

o

o

○

○

o

0

o

o

o

o

○

o

o 


\section{蛋 Portland State}

\section{Question about customer preference and changing existing features}

Below are a list of already existing product features that the team could increase or decrease during product design. In which way would changing these features impact customer preference (i.e. how much customers like the product)?

If you judge that customers will like UniCleanBot moderately better by increasing the suction power of UniCleanBot, you need to place the slider on the corresponding level, Moderately better.

$\begin{array}{lccccccc} & \begin{array}{c}\text { Much } \\ \text { worse }\end{array} & \begin{array}{c}\text { Moderately } \\ \text { worse }\end{array} & \begin{array}{c}\text { Slightly } \\ \text { worse }\end{array} & \begin{array}{c}\text { About the } \\ \text { same }\end{array} & \begin{array}{c}\text { Slightly } \\ \text { better }\end{array} & \begin{array}{c}\text { Moderately } \\ \text { better }\end{array} & \begin{array}{c}\text { Much } \\ \text { better }\end{array} \\ \begin{array}{l}\text { Increase of Battery Capacity } \\ \text { Increase of Suction Power }\end{array} & 0 & 0 & 0 & 0 & 0 & 0 & 0 \\ \begin{array}{l}\text { Increase of Water Tank } \\ \text { Capacity }\end{array} & 0 & 0 & 0 & 0 & 0 & 0 & 0 \\ \begin{array}{l}\text { Decrease of Charging Time } \\ \text { Increase of Dustin Bin }\end{array} & 0 & 0 & 0 & 0 & 0 & 0 & 0 \\ \begin{array}{l}\text { Capacity } \\ \text { Lowering Net Weight }\end{array} & 0 & 0 & 0 & 0 & 0 & 0 & 0 \\ \text { Lowering Profile Height } & 0 & 0 & 0 & 0 & 0 & 0 & 0\end{array}$




\section{面 Portland State}

This is the last page of the first survey. Thanks for your valuable input!

The workshop is scheduled at 9:00am on Feb. 23rd. I am looking forward to seeing you there. I also have a favor to ask: if possible, please bring your own laptop for some practice during the workshop. 


\section{Survey 2}

\section{Portland State \\ UNIVERSITY}

Introduction

This is the second survey to ask your understanding of the product system in UniCleanBot after the individual mental model development activity.

If you are ready, please go to the next page.

This part is identical with Question 1, Question 2, and Question 3 of Survey 1.

Please see Survey 1 relationships between each product design factor and customer preference of UniCleanbot improve as a result of the modeling activity?

A great deal

○

$\mathrm{O}$
Somewhat

O
Very Little

O
Not at All

O

\section{艿 Portland State}

How helpful was the modeling activity for you to improve your understanding of causal relationships between each product design factor and customer preference of UniCleanbot?
A Great Deal
Quite a Bit
Somewhat
Very Little
Not at All

O

O

O

O

0 


\section{P Portland State}

Gratitude

This is the last page of the second survey. Thanks for your valuable input on the research. 


\title{
Survey 3
}

\section{Portland State}

UNIVERSITY

\section{Introduction}

This is the 3rd survey to ask your understanding of the product system in UniCleanBot after the group mental model

development activity

If you are ready, please go to the next page.

This part is identical with Question 1, Question 2, and Question 3 of Survey 1.

Please see Survey 1

\section{क Portland State}

In comparison to what you understood before group discussion, how much did your understanding of causal

relationships between each product design factor and customer preference of UniCleanbot improve as a result of the group discussion?

A great deal

Quite a Bit

Somewhat

Very Little

Not atAll

O

O

O

O

○

\section{P Portland $\int_{\text {UNIVERITY }}$ State}

Please indicate your level of agreement on the last group map resulted from the group discussion.

Strongly agree

O
O

○

○

\begin{abstract}
○
\end{abstract}




\section{P Portland State}

Gratitude

This is the last page of the third survey. Thanks for your valuable input on the research. 
Appendix E - Recruiting Material

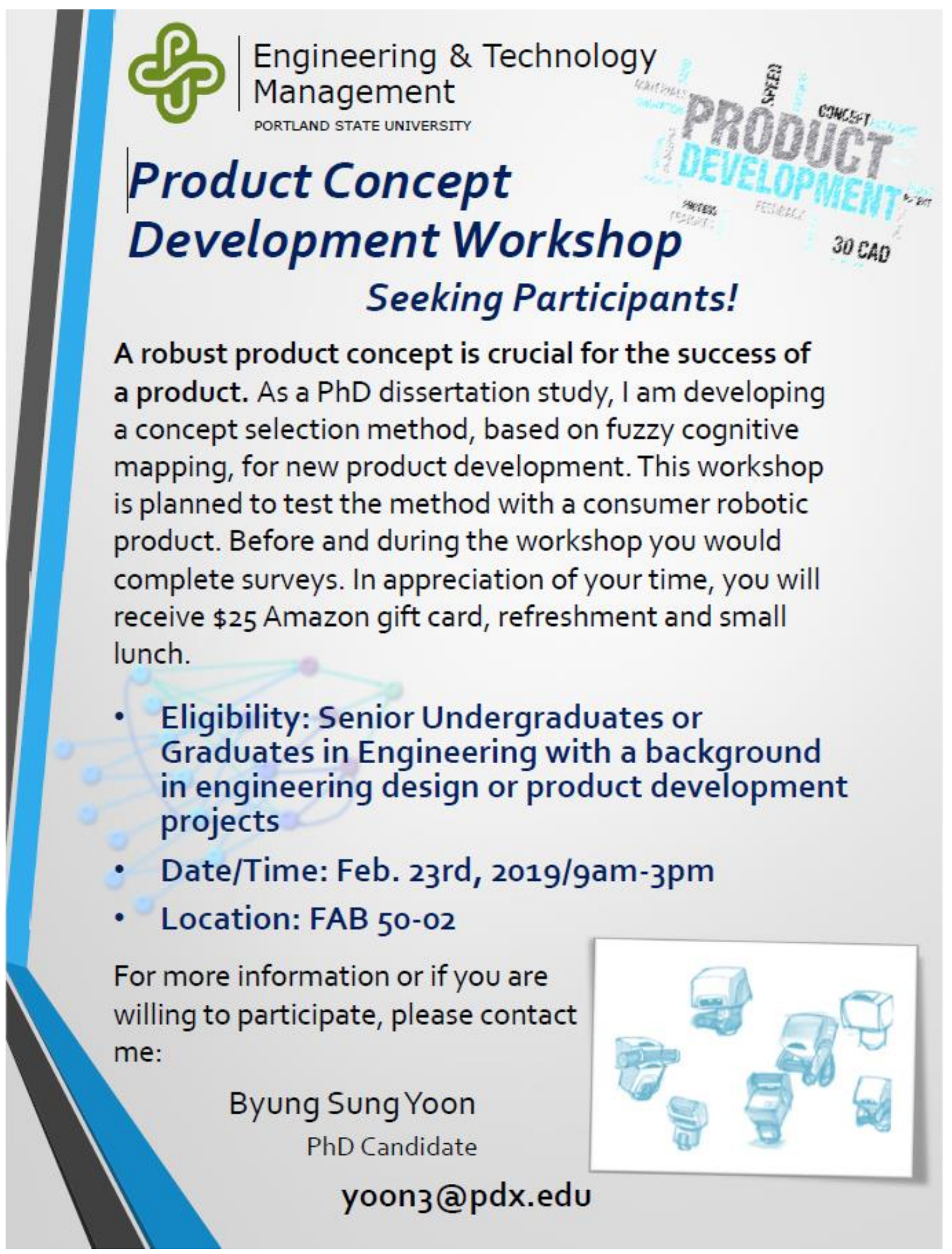




\section{Appendix F - Screening Survey}

Thank you for your willingness to participate in the Product Concept Development Workshop on Feb. 23rd, this Saturday. Prior to the workshop, we'd love to hear from you about your educational experience related to the project.

\section{Screening_Question}

For this study, we require that participants have at least junior-level engineering knowledge. Have you completed an engineering degree program up to the start of junior-level classes in a college?

Yes

No 
Thanks for your answer. I am pleased to inform that you are qualified for this experiment.

\section{CONSENT}

You are making a decision whether to participate in this study. Your signature below indicates that you have read the information provided (or the information was read to you). By signing this consent form, you are not waiving any of your legal rights as a research participant.

You have had an opportunity to ask questions and all questions have been answered to your satisfaction. By signing this consent form, you agree to participate in this study. A copy of this consent form will be provided to you.

If you have any questions regarding to the contents below or this survey, please contact the representative researcher (Byung Sung Yoon; yoon3@pdx.edu).

Do you consent to participate in this research experiment?

I agree

Thanks for your agreement of the consent form. Please let us know your contact information for follow-up.

email

Phone (Optional) 
Thanks for your agreement of the consent form. We want to know your educational experience more in order to analyze the demographic information of this experiment. Please answer the five questions following.

What field did you do the majority of your coursework in?

Mechanical or Materials Engineering or equivalent

Electrical and Computer Engineering or equivalent

Civil and Environmental Engineering or equivalent

Computer Science

Other, please specify 
For the discipline chosen above, what best describes your current academic level?

I am an undergraduate student in this field (junior or beyond)

I am a master's student in this field

I am a Ph.D. student in this field

I have already graduated. My highest degree is 
Please select all product development related courses you have taken or are currently enrolled in.

\section{ETM 543/643 Front End Management for NPD}

ETM 547/647 New Product Development

ETM 549/649 Managing Technological Innovation

ETM 555/655 Technology Marketing

ETM 556/556 User Centered Innovation

ME 491 Design Process

ME 492 Conceptual Design Project

ME 493 Detailed Design Project

ECE 411 Industry Design Processes

Others (Please indicate the course name) 
We want to learn more about your practical, hands-on experience with product design or development projects. Practical experience can be acquired at school (e.g. capstone projects), at work, as a volunteer (e.g. in student clubs), through hobbies, or the Beta Project. Projects are focused at a specific product or design. It does not matter if they were completed.

I have not yet participated in a product design project.

I have participated in one product design project.

I have participated in more than two but less than four product design projects.

I have participated in more than four product design projects.

I am responsible for leading product design projects and have lead at least four.

Is there anything else you would like us to know about your engineering experience? Please write a few words. You can also link to your Linkedln profile (optional). 
The workshop is scheduled from 9am to 3pm on (TBD), Saturday. Are you available to participate in the workshop?

\section{Yes}

No

During the workshop, small lunch (e.g. sandwich) will be served. If you have any food restriction, please indicate it.

Thank you for taking the time to complete this survey. You will receive a follow-up email instructing the following steps and logistics information within a few days. 


\section{Appendix G - Workshop Handout \\ Handout for Product Concept Development Workshop}

Date/Time: February $23^{\text {rd }}$, 2019/9AM-3PM

Location: FAB 12 ETM RISE Conference Room in $4^{\text {th }}$ Ave. Building

\section{Objectives of Workshop}

- To develop product concepts for a target product, a robotic floor cleaner (called UniCleanBot) with novel approach.

- To understand participants' understanding of the target product concepts in the form of mental models

\section{$\underline{\text { Research Information }}$}

As a $\mathrm{PhD}$ dissertation study, this research presents novel method, based on cognitive mapping, that allows teams systematically, holistically, and iteratively assess alternative product concepts and their respective impact on customer value by modeling them as combinations of product design factors. Teams can thus identify and select product concepts that achieve high customer value, given existing constraints. The backbone of the method is fuzzy cognitive mapping. It allows the quantitative representation of group mental models from engineering, which represent knowledge of interdependencies between product features, technologies, and project objectives, and from marketing and future product users, which represent knowledge of interdependencies between features, benefits, and value.

\section{$\underline{\text { Notice }}$}

- Our published data will not identify you as the respondent.

- Any recording (audio/video) during the workshop will be used for only this research and not be published!

- There are risks of stress, emotional distress, inconvenience and possible loss of privacy and confidentiality associated with participating in a research study.

- During your participation, there are not any significant risks related to participation 
in this study. However, you may feel some burden of joining the workshop. In addition, you may feel vulnerable during or after the workshop if you share their experiences with other participants and researchers.

- To mitigate these potential risks, the researchers will encourage you to inform any worry or hardship of joining the workshop and not to share any vulnerable and personal information with other participants and researchers during the workshop.

- Your participation in this study is completely voluntary. You have the right to choose not to participate or to withdraw your participation at any point in this study without penalty or loss of benefits to which you are otherwise entitled.

- If you have questions regarding your rights as a research participant, you may call the PSU Office for Research Integrity at (503) 725-2227 or (877) 480-4400. The ORI is the office that supports the PSU Institutional Review Board (IRB). The IRB is a group of people from PSU and the community who provide independent oversight of safety and ethical issues related to research involving human participants. For more information, you may also access the IRB website at: ttps://sites.google.com/a/pdx.edu/research/integrity

\section{Workshop Plan}

\begin{tabular}{|c|c|c|}
\hline Time & Activity Item & Product of Activity \\
\hline 9:00 AM & \multicolumn{2}{|l|}{ Icebreaking } \\
\hline $9: 15 \mathrm{AM}$ & Welcome, Introduction and Research Overview & Reminding the target product \\
\hline $9: 30 \mathrm{AM}$ & $\begin{array}{l}\text { Introduction Fuzzy Cognitive Mapping } \\
\text { and Preliminary Study }\end{array}$ & $\begin{array}{l}\text { Clear understanding of FCM, as the core } \\
\text { technique of CDRM }\end{array}$ \\
\hline 10:00 AM & \multicolumn{2}{|l|}{ Review PDFs } \\
\hline $10: 30 \mathrm{AM}$ & $\begin{array}{l}\text { Develop Individual Maps } \\
\text { · (Each Participant) Develop own individual map }\end{array}$ & Individual Maps \\
\hline $11: 30 \mathrm{AM}$ & Tea / Coffee Break & Experiment Survey II \\
\hline $11: 45 \mathrm{AM}$ & $\begin{array}{l}\text { Develop PDF Map } \\
\text { · Develop a PDF Map as a group map }\end{array}$ & Group Map (PDF Map) \\
\hline $12: 30 \mathrm{AM}$ & \multicolumn{2}{|l|}{ Lunch Break } \\
\hline 13:00 AM & $\begin{array}{l}\text { Develop Scenarios } \\
\text { · Develop } 3 \text { to } 5 \text { scenarios }\end{array}$ & 3 to 5 Tentative Scenarios \\
\hline 14:00 PM & \multicolumn{2}{|l|}{ Demonstration of FCM Simulation } \\
\hline $14: 30 \mathrm{PM}$ & Review Results of Workshop & $\begin{array}{l}\text { Experiment Survey III } \\
\text { Feedback of the Simulation Results and } \\
\text { Procedure of Workshop }\end{array}$ \\
\hline 15:00 PM & Closing Comments and Adjourn & \\
\hline
\end{tabular}




\section{Glossary}

Product

Feature

Function

Benefit

Product Design

Factors (PDFs)

Product Concept

Customer Preference

Mental Model /

Worldview something sold by an enterprise to its customers such as a good, a service or knowledge, as a bundle of attributes

a physical solution fulfilling a customer problem or a need

something that the product must do or work to meet a customer need

a way in which one or more features of the product provide a definable advantage, improvement, or satisfaction for customers

sub-sets of product attributes that engineering teams can control, such as a product's form, function, and technical specifications. Non-technical product attributes that are outside of the control of engineering, such as brand image, advertising, packaging, and distribution are excluded from the study unless they themselves impact design factors.

a description of the form, function, and features of a product as a set of specifications, an analysis of competitive products, and an economic justification of the projects

the subjective tastes of individual consumers, measured by their satisfaction with those items

a combination of the individual's subjective perceptions, concepts, ideas and perceived system status /

a comprehensive conception or apprehension of the world especially from a specific standpoint 


\section{Fuzzy Cognitive Mapping}

\section{Introduction}

Fuzzy cognitive mapping (FCM) is a technique for representing systems by mapping subjective causal knowledge on digraphs with qualitative and everyday language. FCM was suggested to capture the understanding of uncertain cause and effect relationships of knowledge with adopting the neural network theory, which have gained popularity and been adopted in variety of fields such as engineering, business, medical science, environmental science, and social science. In particular, FCM research related to applications in NPD have been carried out actively.

\section{Advantages}

FCM has several advantages:

- Firstly, as a visualization technique to document and compute human knowledge with language for daily use with semi-quantitative networks, FCM enables to model the system of human perception about a specific topic with relatively easy and various ways such as interviewing, having group sessions and analyzing context contents of literature.

- Secondly, modeling the knowledge of groups is available to be drawn by synthesizing individual maps which individuals in groups developed without any restriction. In addition, after modeling, FCM is available to process dynamic change like modifying or adding additional information on a map.

- Lastly, it is possible to capture cognitive difference between maps created by individuals and groups with comparative analysis. Therefore, FCM is available to capture subjective knowledge of non-technical factors and to present the complex interconnected cause-effect relationships of the product design factor architecture graphically with a direct graph. 


\section{Mathematical Expression}

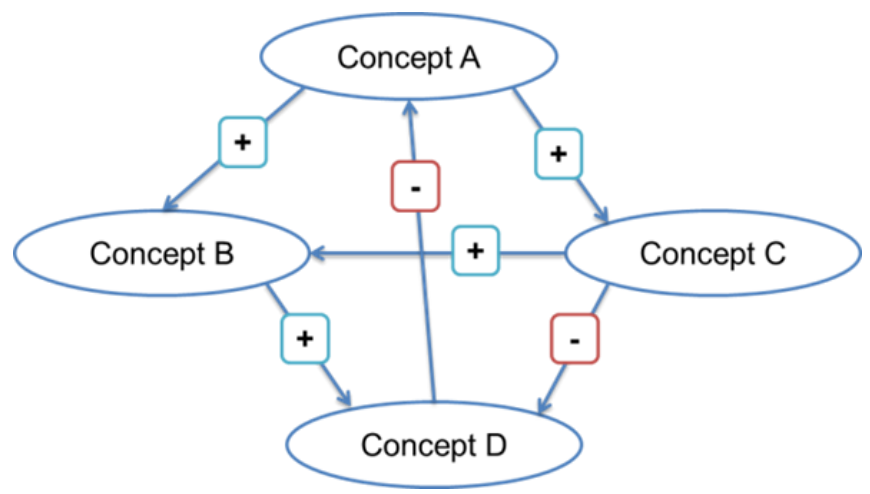

An Example of a FCM

As shown in the picture above, if the concept B (a "passive" concept against the concept A), increases when the concept A (an "active" concept against the concept B) increases, the relationship between the two concepts is positive. Otherwise, if concept D decreases when concept $\mathrm{C}$ increases, the relationship is negative. In this model, the corresponding adjacency matrix of above network is represented like below;

$$
E=\left[\begin{array}{cccc}
0 & +1 & +1 & 0 \\
0 & 0 & 0 & +1 \\
0 & +1 & 0 & -1 \\
-1 & 0 & 0 & 0
\end{array}\right]
$$

In order to investigate the change of each concept caused by a specific decision or a scenario, a state vector which has one row and $\mathrm{n}$ (the number of concepts) columns is multiplied by the adjacency matrix. For instance, if the concept $\mathrm{A}$ is only activated while others are turned off. The initial state vector is like below;

$$
S_{0}^{T}=\left[\begin{array}{llll}
1 & 0 & 0 & 0
\end{array}\right]
$$

From the neural network theory, squashing functions (or thresholds) in simulating FCMs are used to delineate human logical process. In other words, a stimulus to a concept (or node) should be strong enough to activate the concept or to generate an output signal from the concept. To depict this process, squashing functions are applied in modeling FCMs. There are several types of squashing functions such as binary, linear, sigmoid and hyperbolic tangent functions. These squashing functions are: 
- Binary function: $f(x)=\left\{\begin{array}{cc}-1 & \text { for } x<0 \\ 0 & \text { for } x=0 \\ 1 & \text { for } x>0\end{array}\right.$

- Linear function:

$$
f(x)=\left\{\begin{array}{cc}
-1 & \text { for } x \leq-1 \\
x & \text { for }-1<x<1 \\
1 & \text { for } x \geq 1
\end{array}\right.
$$

- Sigmoid function: $f(x)=\frac{1}{1+e^{-\lambda x}}$

- Hyperbolic tangent function: $f(x)=\tanh \lambda x=\frac{e^{\lambda x}-e^{-\lambda x}}{e^{\lambda x}+e^{-\lambda x}}$

where $\lambda$ adjusts the saturation level of a concept activation. A squashing function converts the multiplied values of the adjacency matrix and a former state vector to new state vector:

$$
S_{t}=f\left(E \cdot S_{t-1}\right)
$$

where $t$ is a certain instant. The iteration of this process continues until that the state vector reaches stable status or a stop criterion. Finally, the last state and the behavior of each element in the state vector can be interpreted according to the objective of analysis.

\section{Procedure}

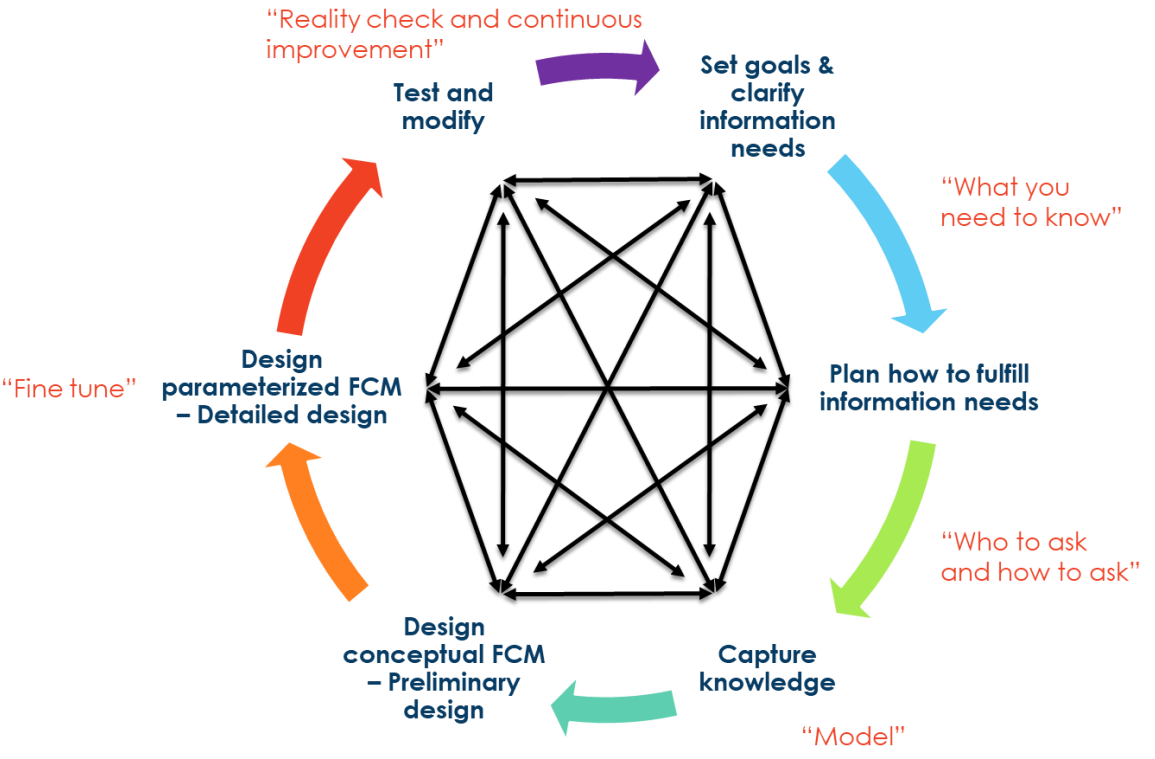




\section{List of Product Design Factors for UniCleanBot}

\begin{tabular}{|c|c|c|c|c|}
\hline $\begin{array}{l}\mathbf{N} \\
\mathbf{0 .}\end{array}$ & $\begin{array}{l}\text { Catego } \\
\text { ry }\end{array}$ & PDF & $\begin{array}{l}\text { Ab } \\
\text { b. }\end{array}$ & Description \\
\hline 1 & Function & $\begin{array}{l}\text { Advanced } \\
\text { Navigation }\end{array}$ & $\begin{array}{l}\text { AN } \\
\text { V }\end{array}$ & $\begin{array}{l}\text { An advanced instrument that determines the position of } \\
\text { UniCleanBot and the route to a particular place }\end{array}$ \\
\hline 2 & Feature & Battery Capacity & $\begin{array}{l}\mathrm{BC} \\
\mathrm{P}\end{array}$ & $\begin{array}{l}\text { The maximum amount of electrical charge stored by a } \\
\text { battery unit to allow the robot to perform its task until } \\
\text { discharged }\end{array}$ \\
\hline 3 & Function & $\begin{array}{l}\text { Battery Charging } \\
\text { Time }\end{array}$ & $\begin{array}{l}\mathrm{BC} \\
\mathrm{T}\end{array}$ & $\begin{array}{l}\text { A time period required to charge up the rechargeable } \\
\text { battery unit with electrical energy sufficiently or fully }\end{array}$ \\
\hline 4 & Benefit & $\begin{array}{l}\text { Cleaning } \\
\text { Performance }\end{array}$ & $\mathrm{CPF}$ & $\begin{array}{l}\text { The degree of performance how well a robot can clean a } \\
\text { specific location being set }\end{array}$ \\
\hline 5 & $\begin{array}{l}\text { Custome } \\
\mathrm{r} \\
\text { Preferen } \\
\text { ce }\end{array}$ & $\begin{array}{l}\text { Customer } \\
\text { Preference }\end{array}$ & CSP & The subjective tastes of individual consumers \\
\hline 6 & Feature & Dustbin Capacity & $\begin{array}{l}\mathrm{DC} \\
\mathrm{P}\end{array}$ & $\begin{array}{l}\text { The maximum amount of dust stored by the dustbin the } \\
\text { robot equipped }\end{array}$ \\
\hline 7 & $\begin{array}{l}\text { Tech. } \\
\text { Alternati } \\
\text { ve }\end{array}$ & $\begin{array}{l}\text { Expandable Corner } \\
\text { Brush }\end{array}$ & $\begin{array}{l}\text { EC } \\
\mathrm{B}\end{array}$ & $\begin{array}{l}\text { A brush enabling to clean dust in corners where the } \\
\text { robot can hardly reach because of its shape or size }\end{array}$ \\
\hline 8 & Feature & $\begin{array}{l}\text { Energy Level } \\
\text { Indicator }\end{array}$ & ELI & $\begin{array}{l}\text { A visible indicator showing the remaining energy } \\
\text { capacity of battery }\end{array}$ \\
\hline 9 & Function & Face Recognition & FRE & $\begin{array}{l}\text { The ability to automatically recognize human faces } \\
\text { based on dynamic facial images is important in security } \\
\text { and surveillance }\end{array}$ \\
\hline 10 & $\begin{array}{l}\text { Tech. } \\
\text { Alternati } \\
\text { ve }\end{array}$ & HEPA Filter & $\begin{array}{l}\mathrm{HE} \\
\mathrm{P}\end{array}$ & $\begin{array}{l}\text { High efficiency Particulate Air Filter for individuals } \\
\text { who are allergic or asthmatic }\end{array}$ \\
\hline 11 & Benefit & $\begin{array}{l}\text { Intelligent Power } \\
\text { Management }\end{array}$ & IPM & $\begin{array}{l}\text { A combination of hardware and software that optimizes } \\
\text { the distribution and use of electrical power in a robot }\end{array}$ \\
\hline 12 & Feature & $\begin{array}{l}\text { Lower Profile } \\
\text { Design }\end{array}$ & $\begin{array}{l}\text { LP } \\
D\end{array}$ & $\begin{array}{l}\text { A thin profile design small enough to fit under the } \\
\text { couch, bed, or any type of furniture in your house }\end{array}$ \\
\hline 13 & Benefit & $\begin{array}{l}\text { Ease of } \\
\text { Maintenance }\end{array}$ & $\begin{array}{l}\text { MT } \\
\mathrm{N}\end{array}$ & $\begin{array}{l}\text { The ease with which a robot can be maintained in order } \\
\text { to repair fault or worn-out components }\end{array}$ \\
\hline 14 & Feature & Net Weight & $\begin{array}{l}\mathrm{NW} \\
\mathrm{T}\end{array}$ & $\begin{array}{l}\text { Actual or estimated weight of a robot without } \\
\text { packaging }\end{array}$ \\
\hline 15 & Benefit & $\begin{array}{l}\text { Operational } \\
\text { Excellence }\end{array}$ & $\begin{array}{l}\text { OP } \\
\mathrm{E}\end{array}$ & $\begin{array}{l}\text { The execution of cleaning more consistently and } \\
\text { reliably with using the robot than other devices }\end{array}$ \\
\hline 16 & Feature & Profile Height & $\begin{array}{l}\mathrm{PH} \\
\mathrm{T}\end{array}$ & $\begin{array}{l}\text { The vertical distance from floor to the highest part of a } \\
\text { robot }\end{array}$ \\
\hline 17 & Function & $\begin{array}{l}\text { Step-climbing } \\
\text { Mechanism }\end{array}$ & $\begin{array}{l}\mathrm{SC} \\
\mathrm{M}\end{array}$ & $\begin{array}{l}\text { A mechanism for enabling the robot to climb stairs and } \\
\text { move from floor to floor }\end{array}$ \\
\hline 18 & Function & $\begin{array}{l}\text { Self-emptying } \\
\text { Dustbin }\end{array}$ & $\begin{array}{l}\text { SE } \\
\mathrm{D}\end{array}$ & $\begin{array}{l}\text { The ability to empty its dusbin automaticlly at the } \\
\text { docking station }\end{array}$ \\
\hline 19 & Benefit & Safety & SFT & $\begin{array}{l}\text { The state of not being dangerous or harmful in using a } \\
\text { robot }\end{array}$ \\
\hline 20 & Function & Speech Recognition & SPR & $\begin{array}{l}\text { The ability of a machine or program to identify words } \\
\text { and phrases in spoken language and convert them to a } \\
\text { UniCleanBot-readable format }\end{array}$ \\
\hline
\end{tabular}




\begin{tabular}{|l|l|l|l|l|}
\hline 21 & Feature & Suction Power & $\begin{array}{l}\text { SP } \\
\text { W }\end{array}$ & $\begin{array}{l}\text { A mechanical power in the form of suction with air } \\
\text { flow, enabling to collect dust or debris into the dustbin }\end{array}$ \\
\hline 22 & Function & Waterproof Design & $\begin{array}{l}\text { WP } \\
\text { D }\end{array}$ & $\begin{array}{l}\text { Design to obtain sealing ability to protect electrical } \\
\text { parts or subsystems against water }\end{array}$ \\
\hline 23 & Feature & $\begin{array}{l}\text { Water Tank } \\
\text { Capacity }\end{array}$ & $\begin{array}{l}\text { WT } \\
\text { C }\end{array}$ & $\begin{array}{l}\text { The maximum amount of clean water by the water tank } \\
\text { the robot equipped for cleaning purpose }\end{array}$ \\
\hline
\end{tabular}


Appendix H - Survey Results

\section{Question 1}
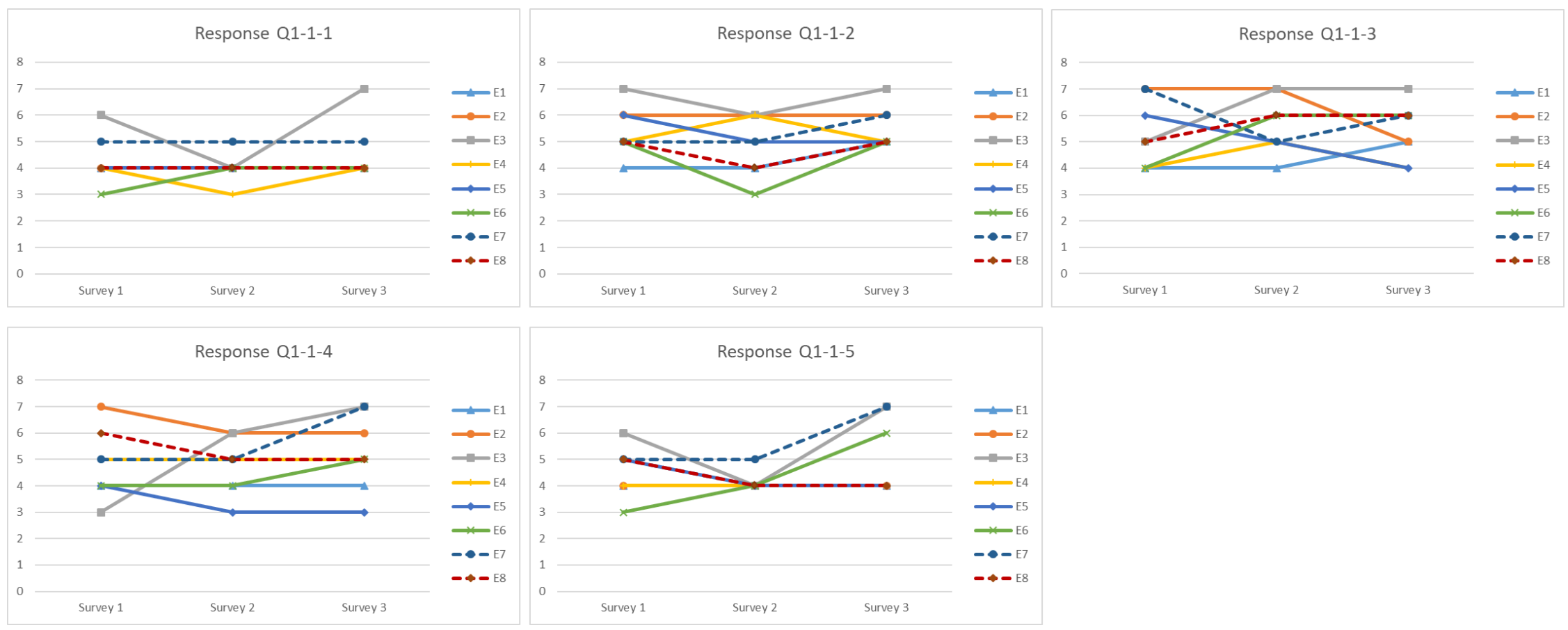


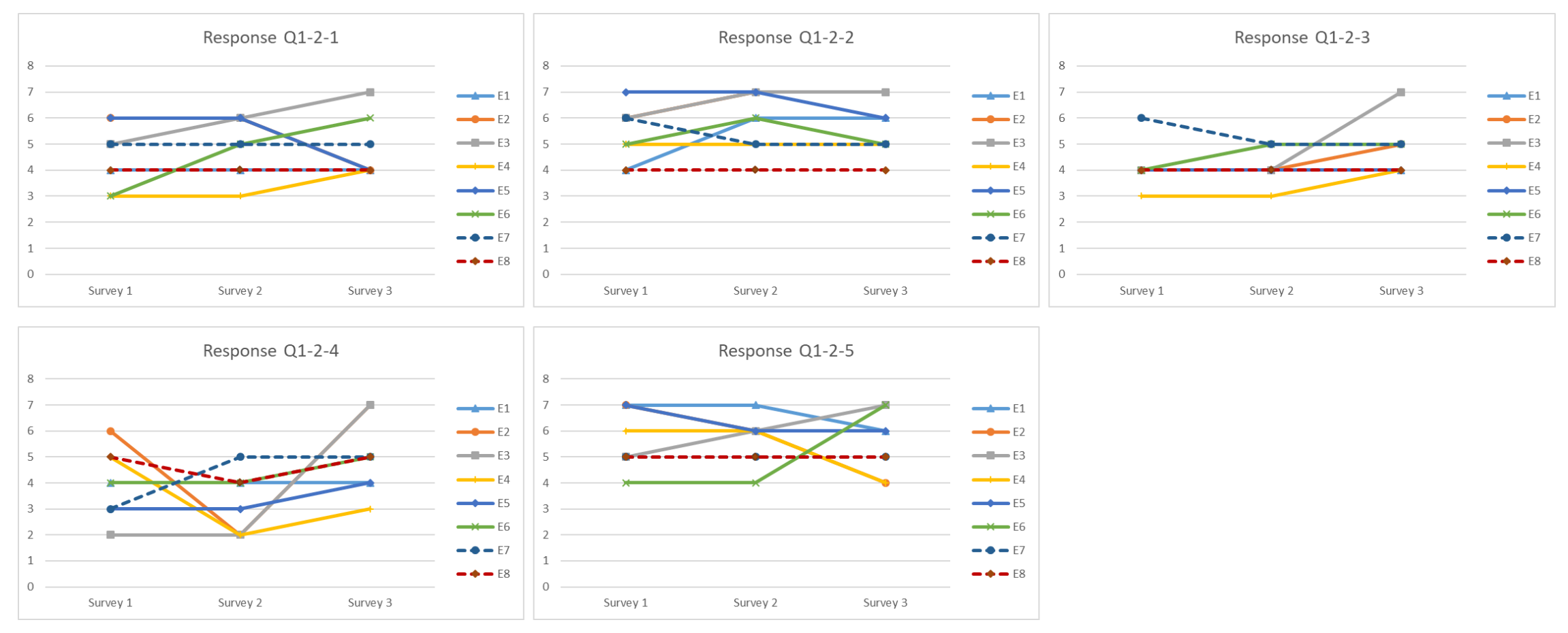




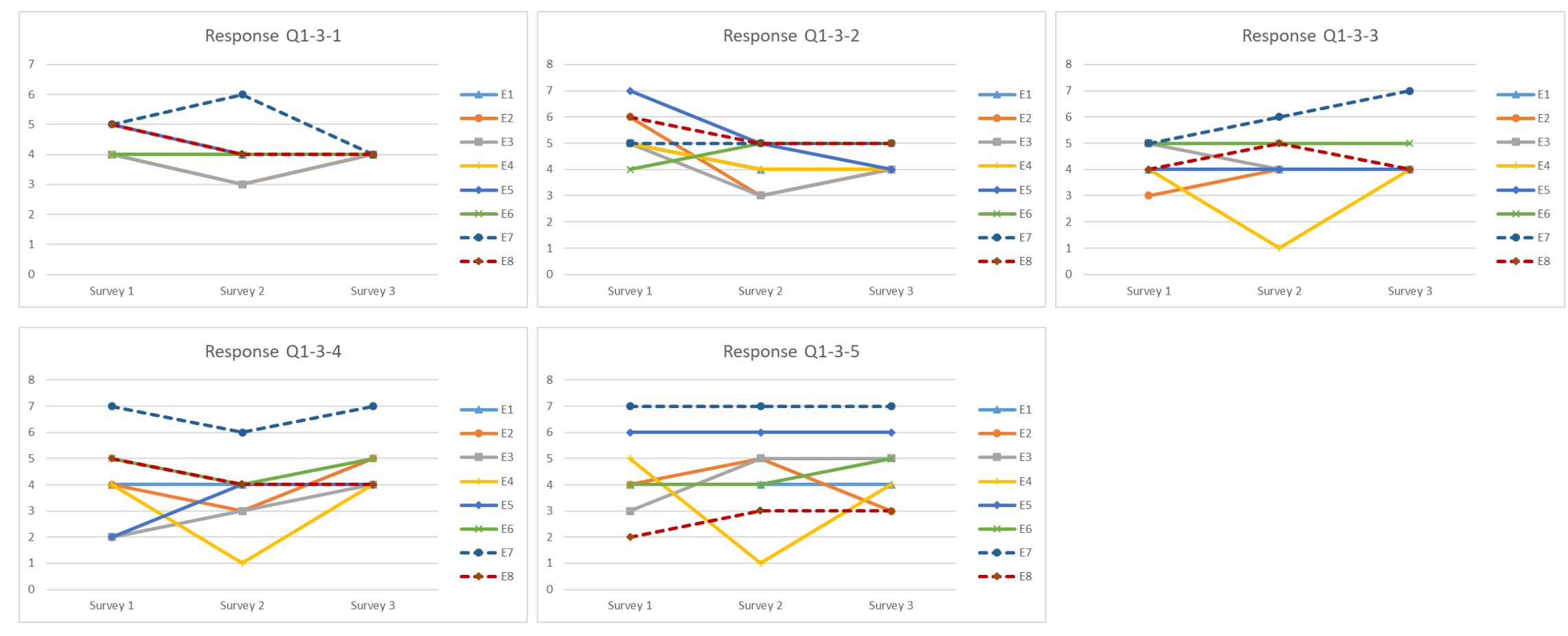




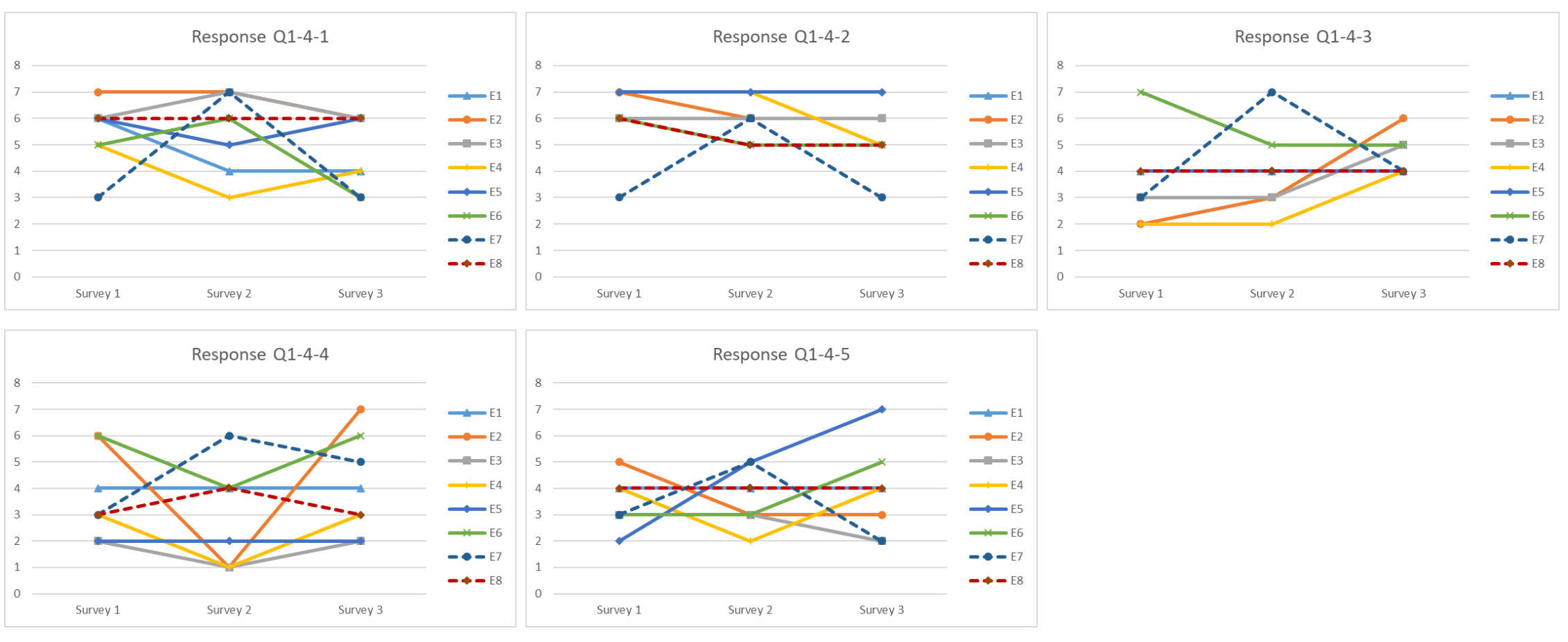




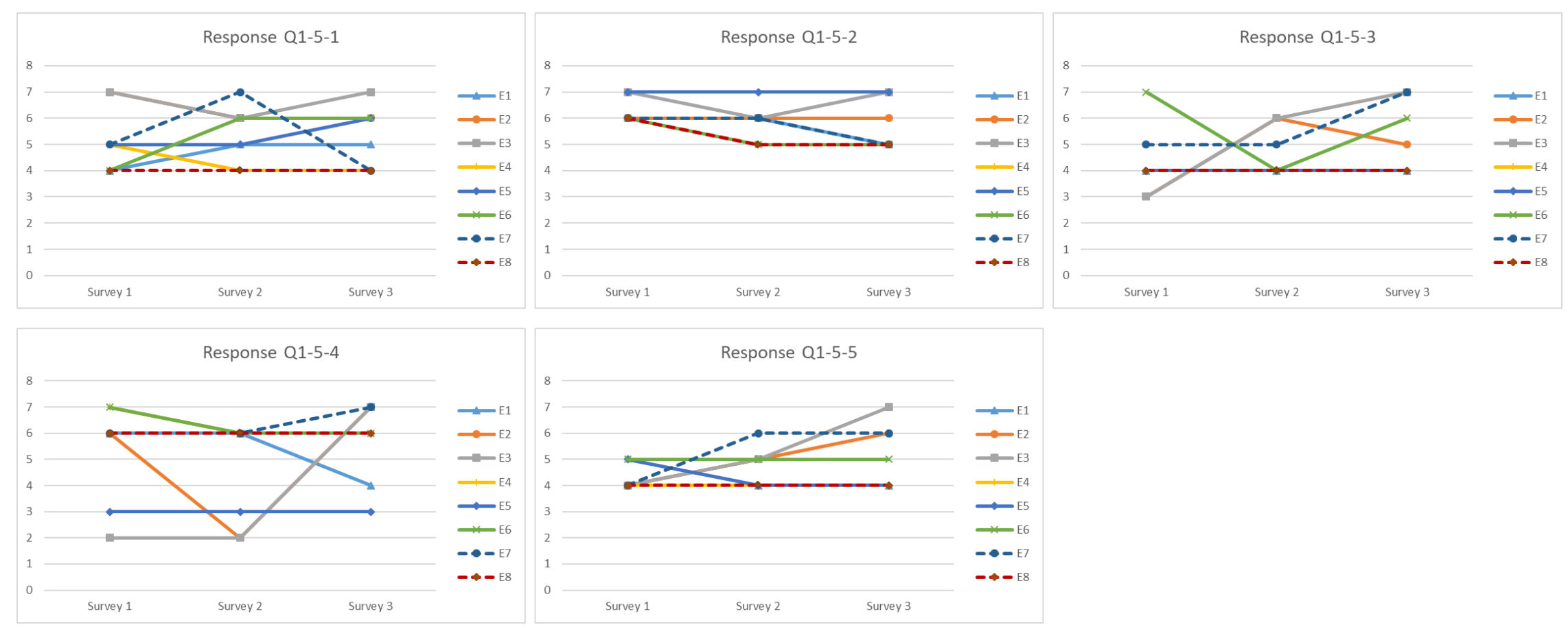




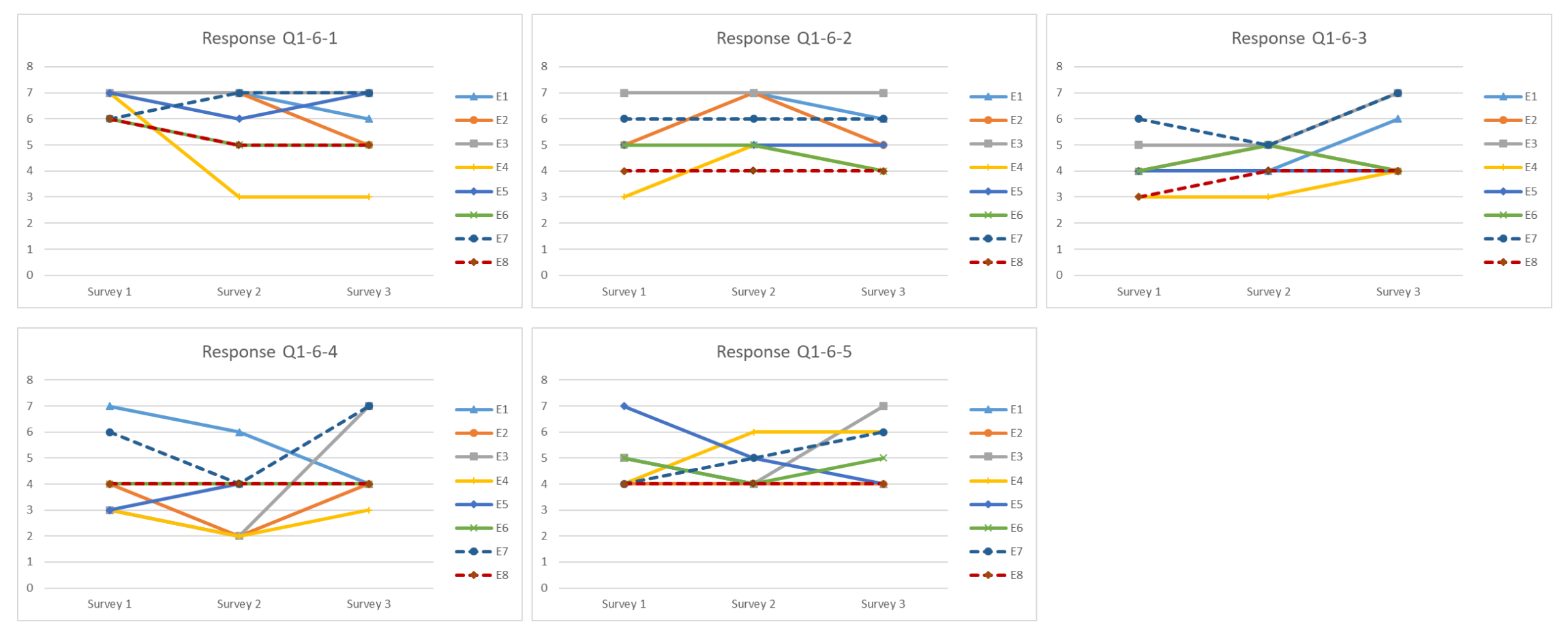




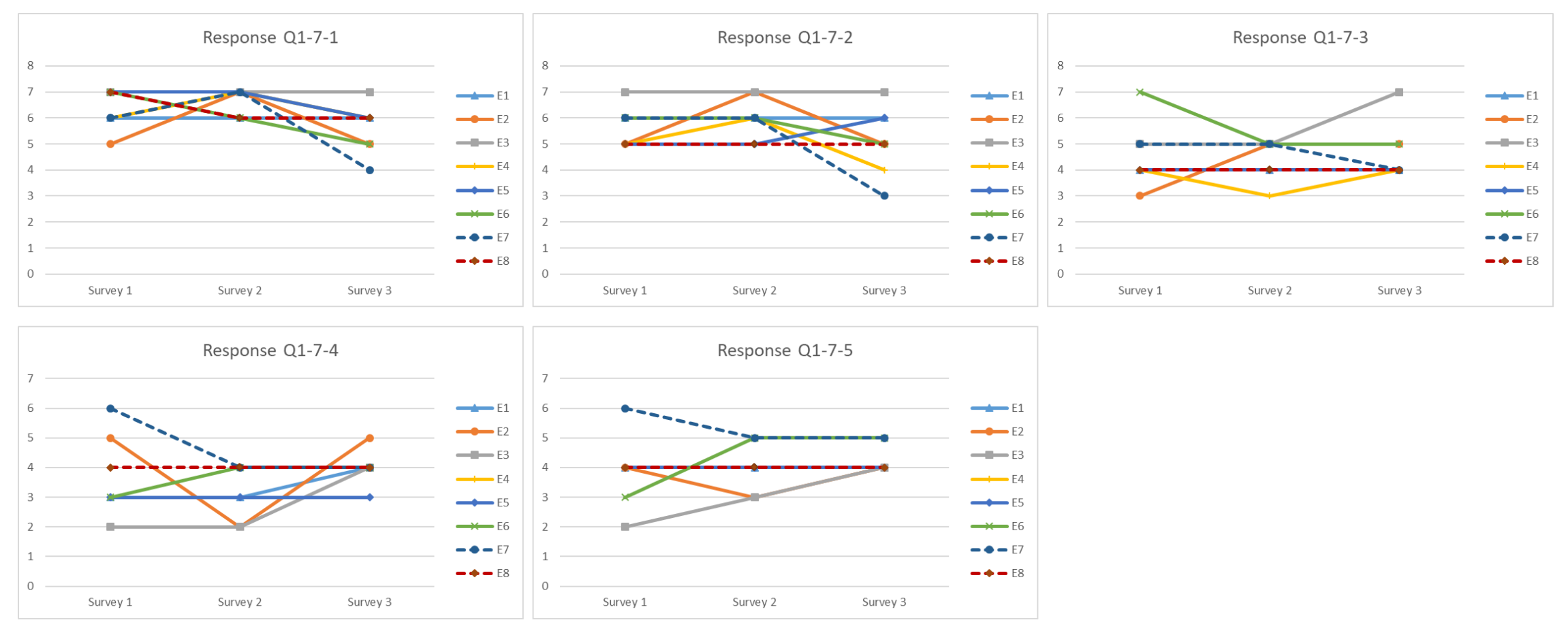




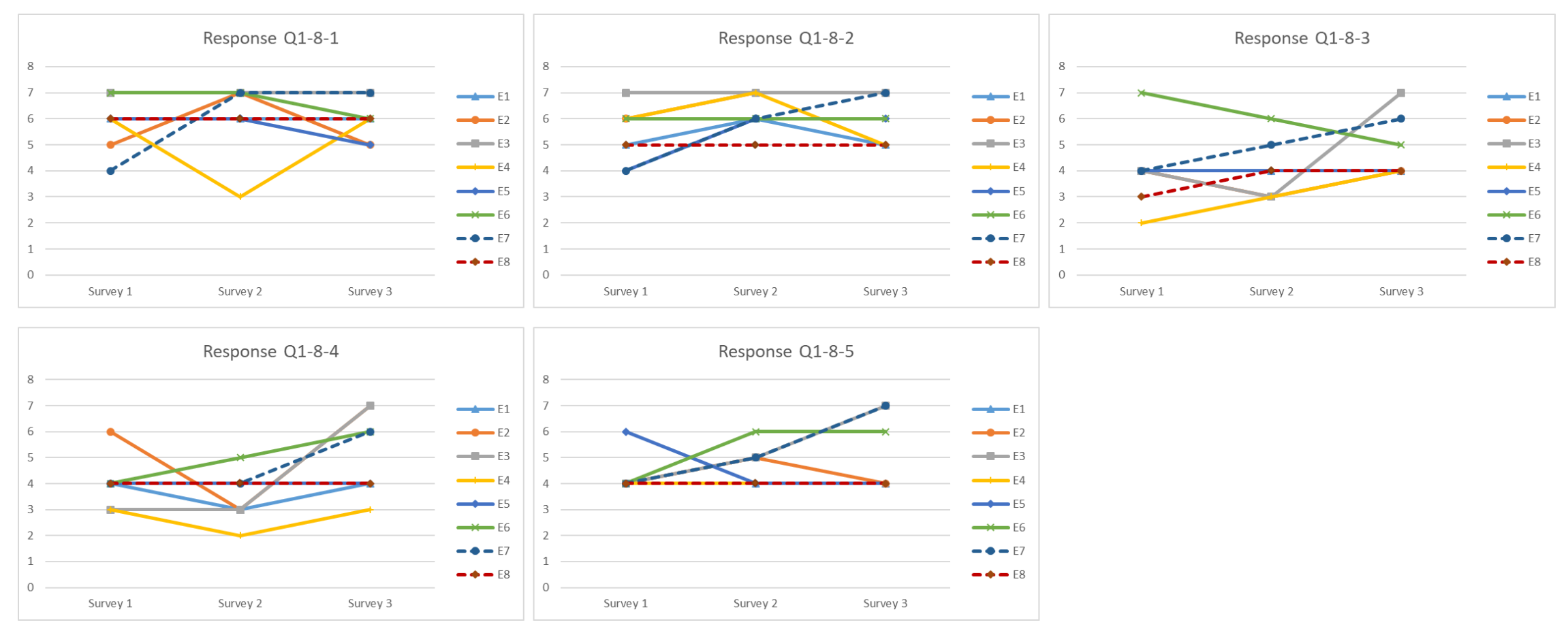




\section{Question 2}
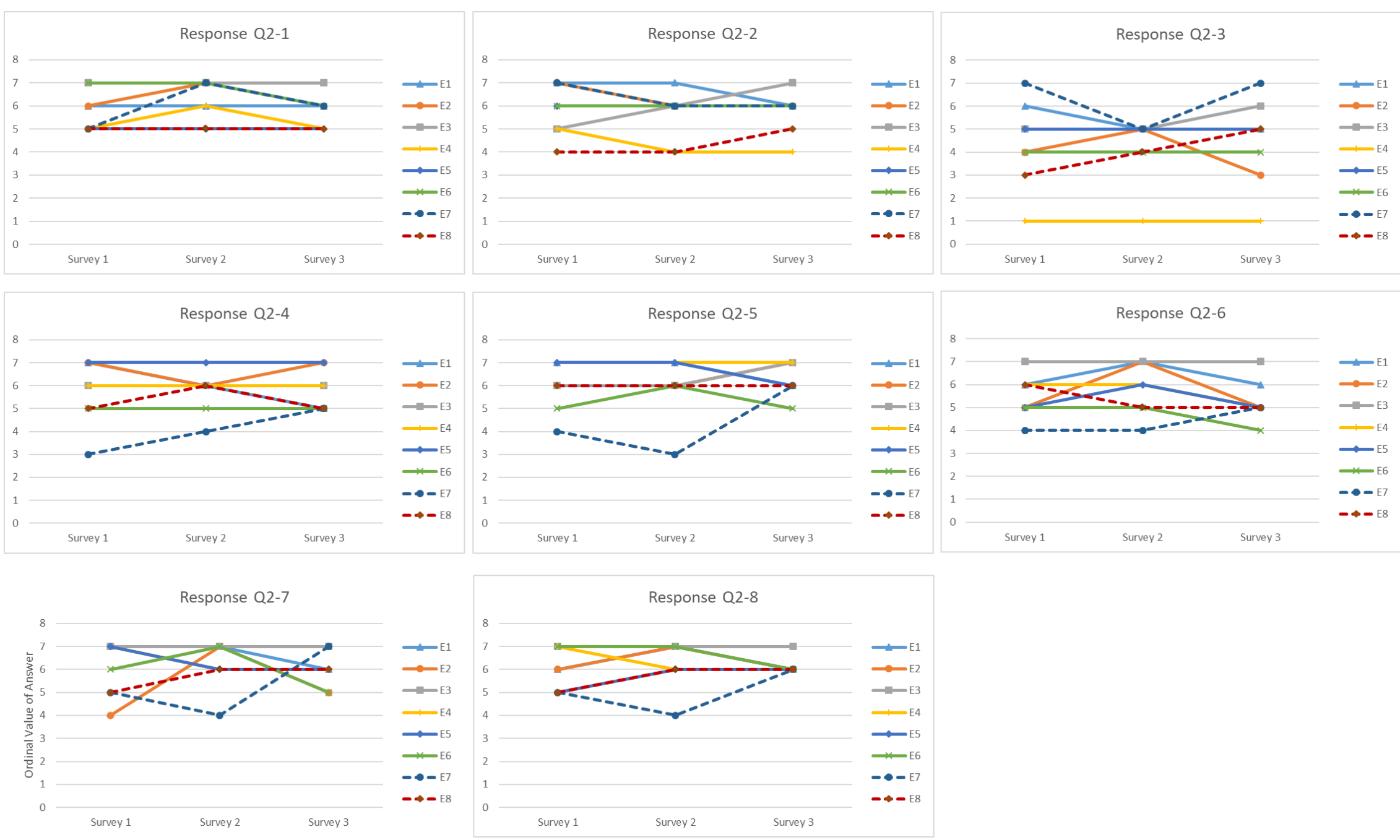

芯 


\section{Question 3}
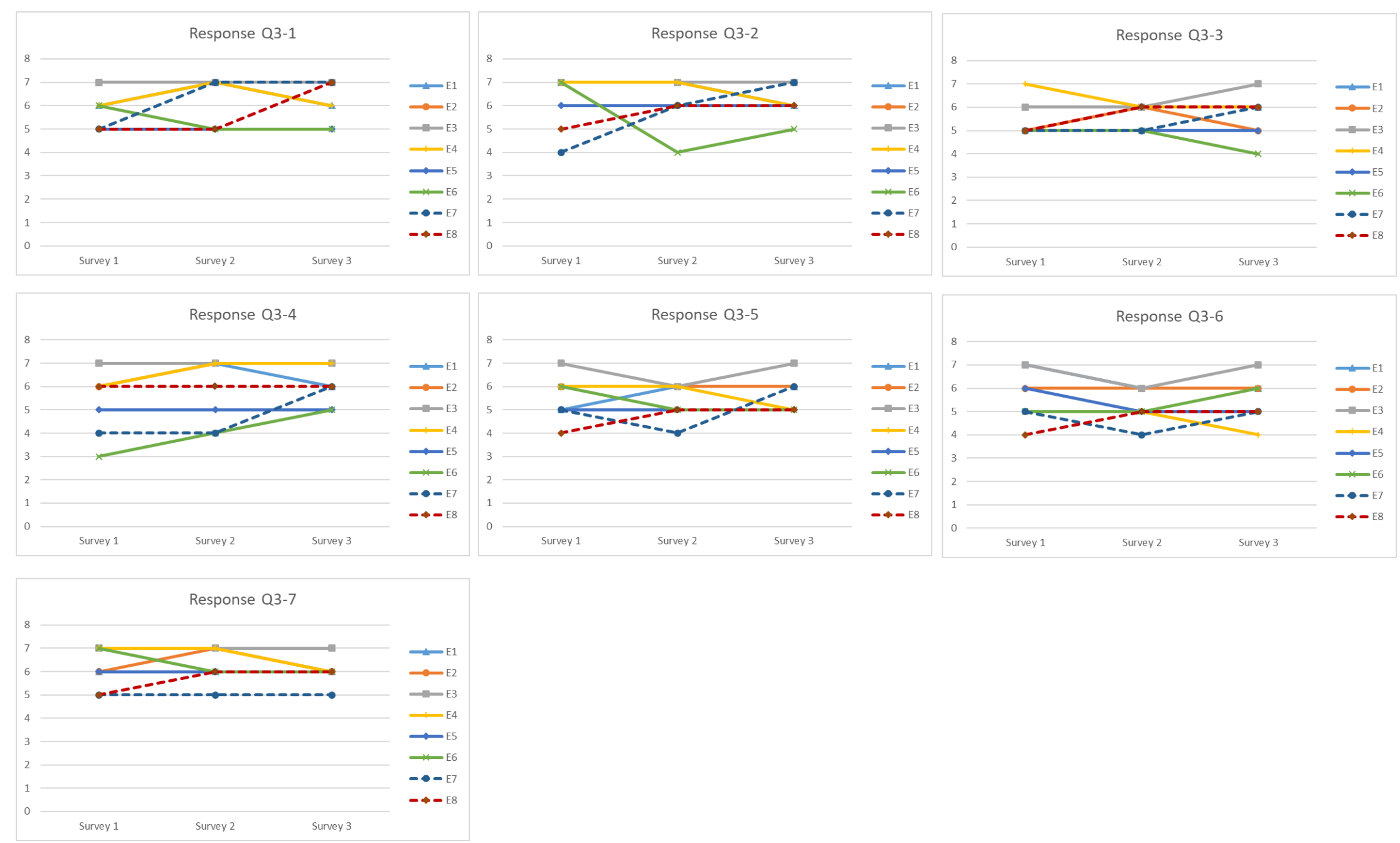

$\tilde{D}$ 


\section{Appendix I - Raw Individual FCMs}

E1

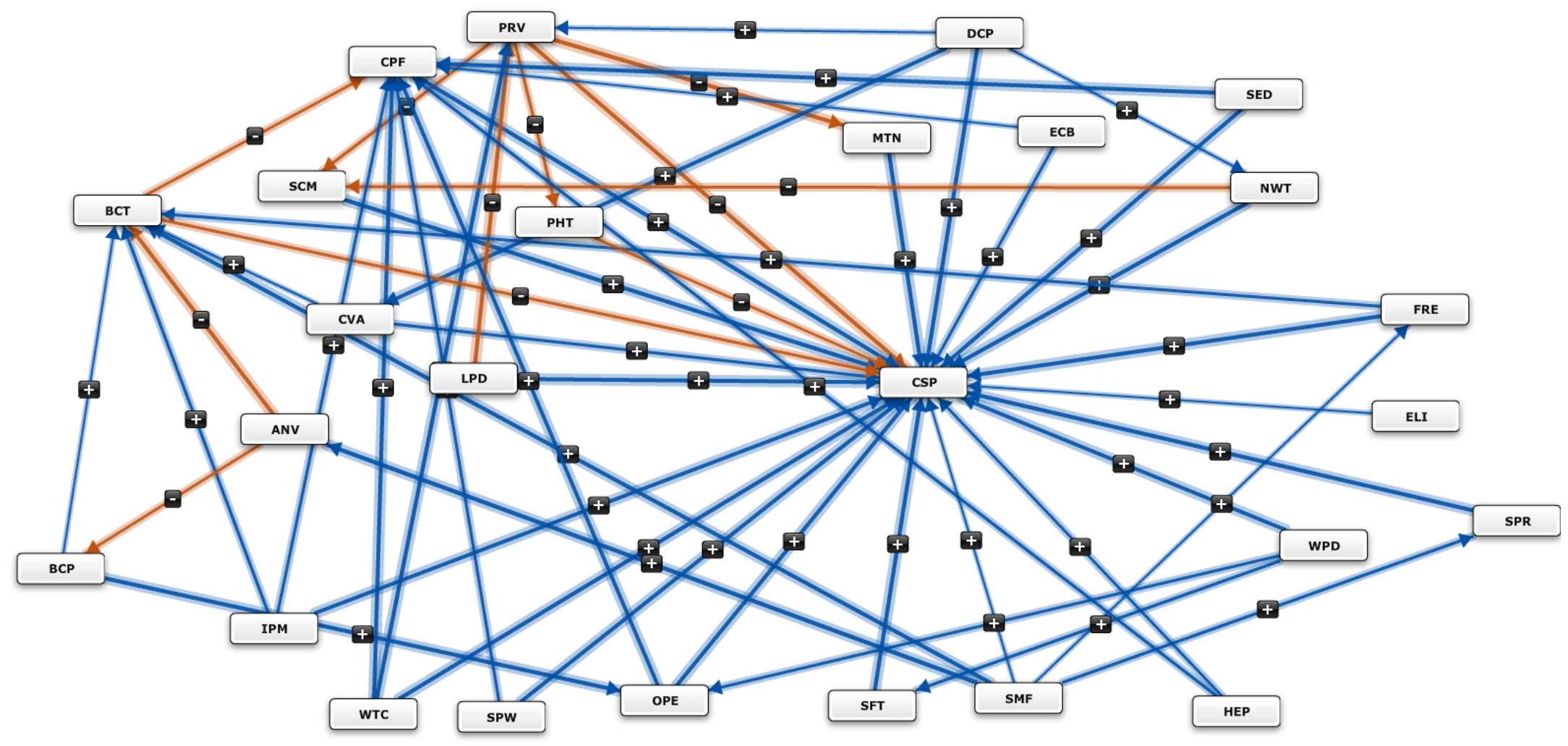




\begin{tabular}{|c|c|c|c|c|c|c|c|c|c|c|c|c|c|c|c|c|c|c|c|c|c|c|c|c|c|c|}
\hline & ELI & FRE & SCM & SED & WPD & CPF & IPM & MTN & SFT & OPE & HEP & ECB & LPD & $\mathrm{BCP}$ & SPW & WTC & $\mathrm{BCT}$ & NWT & PHT & CSP & ANV & SPR & DCP & CVA & PRV & SMF \\
\hline ELI & & & & & & & & & & & & & & & & & & & & 0.43 & & & & & & \\
\hline FRE & & & & & & & & & & & & & & & & & 0.73 & & & 1 & & & & & & \\
\hline SCM & & & & & & & & & & & & & & & & & & & & 0.97 & & & & & & \\
\hline SED & & & & & & 0.87 & & & & & & & & & & & & & & 1 & & & & & & \\
\hline WPD & & & & & & & & & 0.8 & 0.67 & & & & & & & & & & 1 & & & & & & \\
\hline CPF & & & & & & & & & & & & & & & & & & & & 1 & & & & & & \\
\hline IPM & & & & & & 0.7 & & & & & & & & & & & 0.5 & & & 0.9 & & & & & & \\
\hline MTN & & & & & & & & & & & & & & & & & & & & 1 & & & & & & \\
\hline SFT & & & & & & & & & & & & & & & & & & & & 1 & & & & & & \\
\hline OPE & & & & & & 1 & & & & & & & & & & & & & & 1 & & & & & & \\
\hline HEP & & & & & & 0.83 & & & & & & & & & & & & & & 0.77 & & & & & & \\
\hline $\mathrm{ECB}$ & & & & & & 0.37 & & & & & & & & & & & & & & 0.63 & & & & & & \\
\hline LPD & & & & & & & & & & & & & & & & & & & & 1 & & & & & -1 & \\
\hline $\mathrm{BCP}$ & & & & & & & & & & 1 & & & & & & & 0.37 & & & & & & & & & \\
\hline SPW & & & & & & 0.5 & & & & & & & & & & & & & & 1 & & & & & & \\
\hline WTC & & & & & & 0.93 & & & & & & & & & & & & & & 1 & & & & & 0.97 & \\
\hline $\mathrm{BCT}$ & & & & & & -0.5 & & & & & & & & & & & & & & -0.5 & & & & & & \\
\hline NWT & & & -0.77 & & & & & & & & & & & & & & & & & 1 & & & & & & \\
\hline PHT & & & & & & & & & & & & & & & & & & & & -0.77 & & & & & & \\
\hline CSP & & & & & & & & & & & & & & & & & & & & & & & & & & \\
\hline ANV & & & & & & & & & & & & & & -0.7 & & & -1 & & & & & & & & & \\
\hline SPR & & & & & & & & & & & & & & & & & & & & 0.9 & & & & & & \\
\hline DCP & & & & & & & & & & & & & & & & & & 0.47 & & 1 & & & & 1 & 0.4 & \\
\hline CVA & & & & & & & & & & & & & & & & & 0.2 & & & 0.67 & & & & & & \\
\hline PRV & & & -0.83 & & & & & -1 & & & & & & & & & & & -0.77 & -1 & & & & & & \\
\hline SMF & & 0.4 & & & & & & & & & & & & & & & 1 & & & 0.43 & 1 & 0.53 & & & & \\
\hline
\end{tabular}

용 
E2

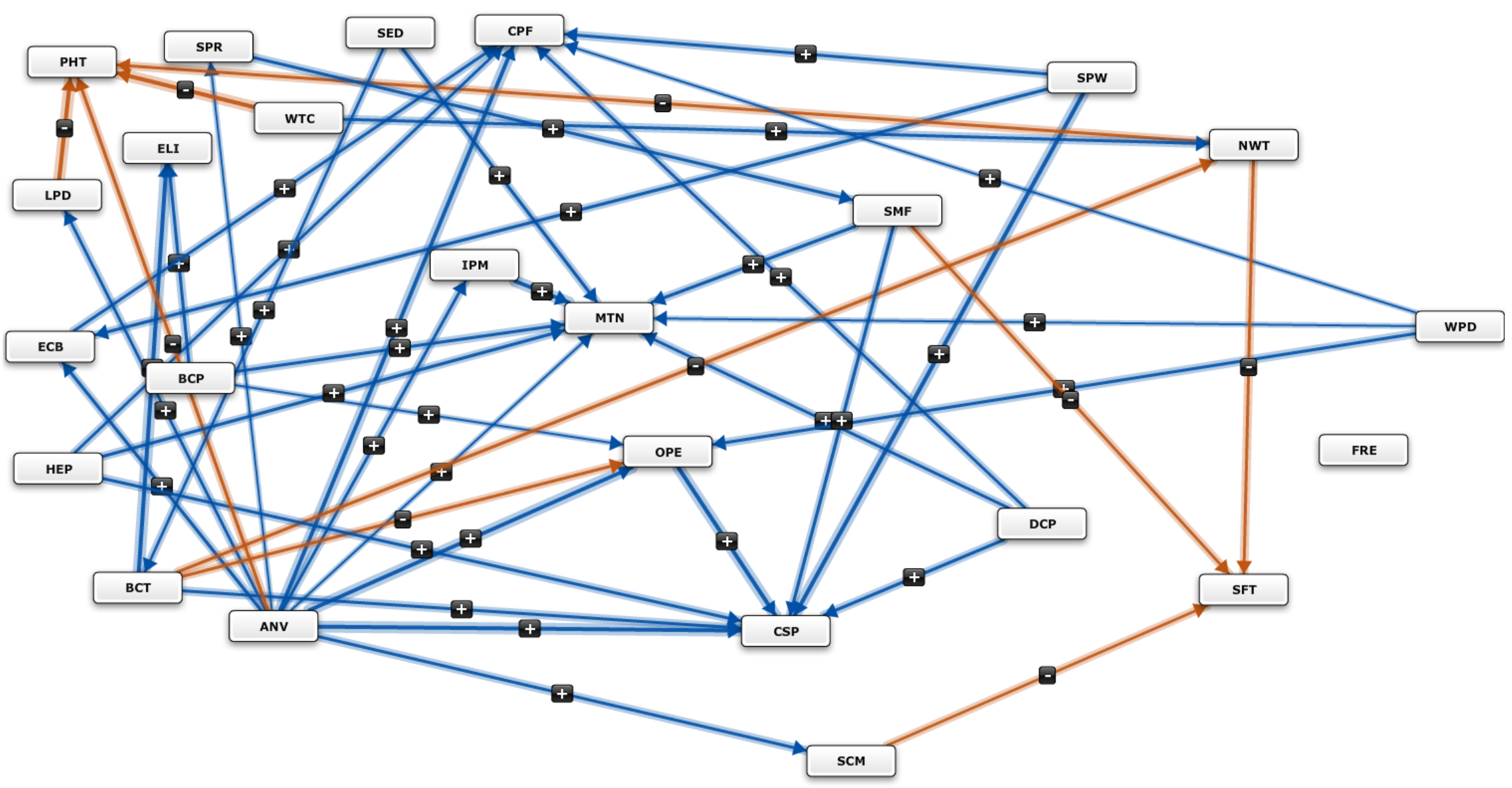




\begin{tabular}{|c|c|c|c|c|c|c|c|c|c|c|c|c|c|c|c|c|c|c|c|c|c|c|c|c|}
\hline & ELI & FRE & SCM & SED & WPD & CPF & IPM & MTN & SFT & OPE & HEP & ECB & LPD & $\mathrm{BCP}$ & SPW & WTC & $\mathrm{BCT}$ & NWT & PHT & CSP & ANV & SPR & DCP & SMF \\
\hline \multicolumn{25}{|l|}{ ELI } \\
\hline \multicolumn{25}{|l|}{ FRE } \\
\hline SCM & & & & & & & & & -0.5 & & & & & & & & & & & & & & & \\
\hline SED & & & & & & & & 0.7 & & & & & & & & & 0.75 & & & & & & & \\
\hline WPD & & & & & & 0.47 & & 0.47 & & 0.54 & & & & & & & & & & & & & & \\
\hline \multicolumn{25}{|l|}{ CPF } \\
\hline IPM & & & & & & & & 0.87 & & & & & & & & & & & & & & & & \\
\hline \multicolumn{25}{|l|}{ MTN } \\
\hline \multicolumn{25}{|l|}{ SFT } \\
\hline OPE & & & & & & & & & & & & & & & & & & & & 1 & & & & \\
\hline HEP & & & & & & 0.75 & & 0.6 & & & & & & & & & & & & 0.67 & & & & \\
\hline$E C B$ & & & & & & 0.77 & & & & & & & & & & & & & & & & & & \\
\hline LPD & & & & & & & & & & & & & & & & & & & -1 & & & & & \\
\hline$B C P$ & 0.77 & & & & & & & 0.75 & & 0.49 & & & & & & & & & & & & & & \\
\hline SPW & & & & & & 0.7 & & & & & & 0.77 & & & & & & & & 1 & & & & \\
\hline WTC & & & & & & & & & & & & & & & & & & 0.67 & -0.9 & & & & & \\
\hline BCT & 0.93 & & & & & & & & & -0.8 & & & & & & & & -0.6 & & 0.6 & & & & \\
\hline NWT & & & & & & & & & -0.5 & & & & & & & & & & -0.8 & & & & & \\
\hline \multicolumn{25}{|l|}{ PHT } \\
\hline CSP & & & & & & & & & & & & & & & & & & & & & & & & \\
\hline ANV & & & 0.6 & & & 1 & 0.55 & 0.49 & & 0.87 & & 0.5 & 0.82 & & & & & & -0.7 & 1 & & 0.44 & & \\
\hline SPR & & & & & & & & & & & & & & & & & & & & & & & & 0.65 \\
\hline DCP & & & & & & 0.7 & & 0.8 & & & & & & & & & & & & 0.77 & & & & \\
\hline SMF & & & & & & & & 0.67 & -0.6 & & & & & & & & & & & 0.72 & & & & \\
\hline
\end{tabular}

$\widetilde{\infty}$ 


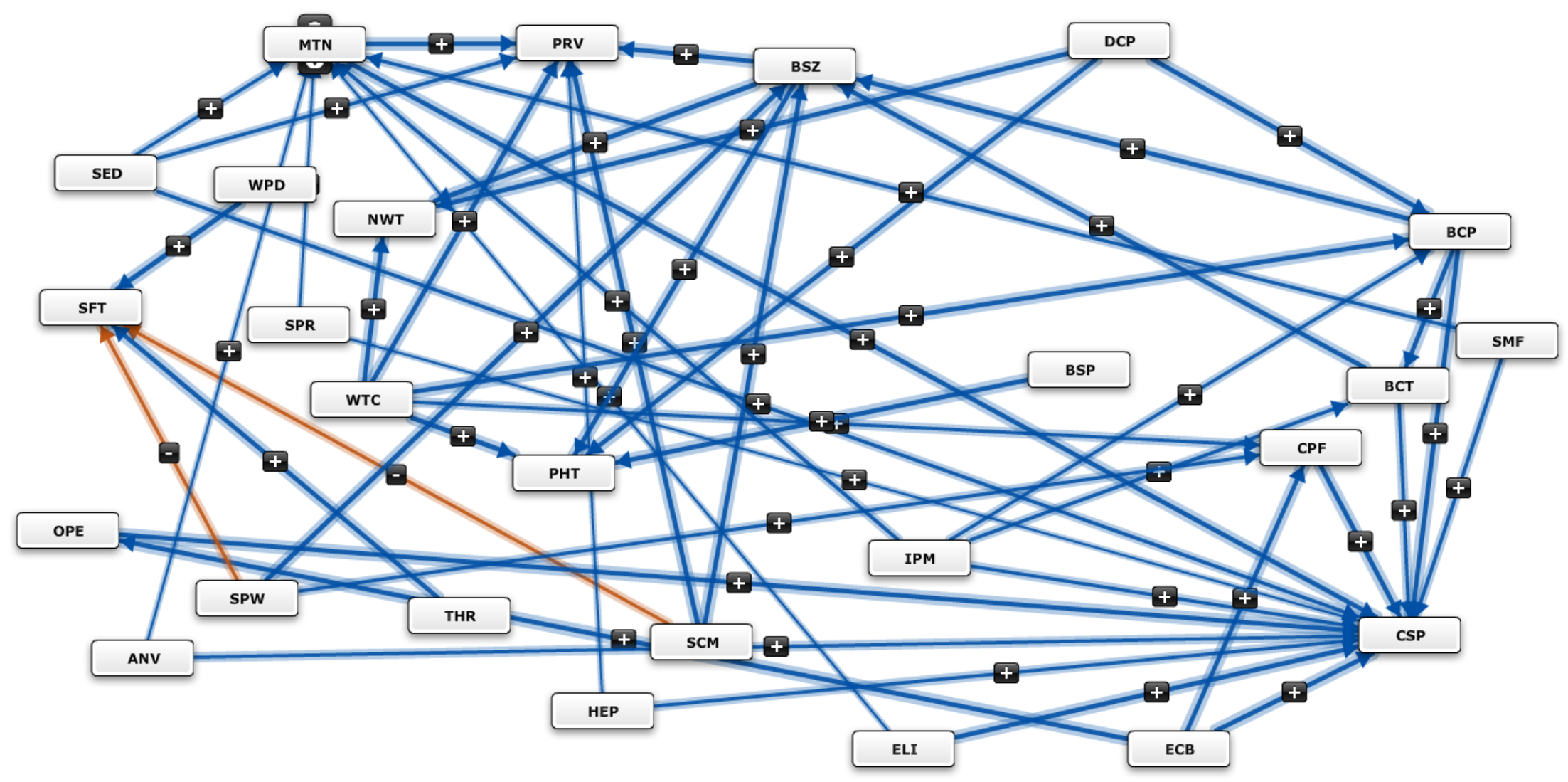

FRE LPD

๕ั 


\begin{tabular}{|c|c|c|c|c|c|c|c|c|c|c|c|c|c|c|c|c|c|c|c|c|c|c|c|c|c|c|c|c|}
\hline & ELI & FRE & SCM & SED & WPD & $\mathrm{CPF}$ & IPM & MTN & SFT & OPE & HEP & $\mathrm{ECB}$ & LPD & $\mathrm{BCP}$ & SPW & WTC & $\mathrm{BCT}$ & NWT & PHT & CSP & ANV & SPR & DCP & BSZ & BSP & PRV & THR & SMF \\
\hline ELI & & & & & & & & 0.17 & & & & & & & & & & & & 1 & & & & & & & & \\
\hline FRE & & & & & & & & & & & & & & & & & & & & & & & & & & & & \\
\hline SCM & & & & & & & & & -0.8 & & & & & & & & & & & & & & & 1 & 1 & 1 & & \\
\hline SED & & & & & & & & 0.83 & & & & & & & & & & & & 0.5 & & & & & & 0.53 & & \\
\hline WPD & & & & & & & & & 1 & & & & & & & & & & & & & & & & & & & \\
\hline CPF & & & & & & & & & & & & & & & & & & & & 1 & & & & & & & & \\
\hline IPM & & & & & & & & 0.53 & & & & & & 0.69 & & & 0.5 & & & 0.53 & & & & & & & & \\
\hline MTN & & & & & & & & & & & & & & & & & & & & 1 & & & & & & 1 & & \\
\hline SFT & & & & & & & & & & & & & & & & & & & & & & & & & & & & \\
\hline OPE & & & & & & & & & & & & & & & & & & & & 1 & & & & & & & & \\
\hline HEP & & & & & & & & & & & & & & & & & & & & 0.81 & & & & & & 0.47 & & \\
\hline ECB & & & & & & 1 & & & & 1 & & & & & & & & & & 1 & & & & & & & & \\
\hline LPD & & & & & & & & & & & & & & & & & & & & & & & & & & & & \\
\hline$B C P$ & & & & & & & & & & & & & & & & & 1 & & & 0.86 & & & & 1 & 1 & & & \\
\hline SPW & & & & & & 0.81 & & & -0.5 & & & & & & & & & & & & & & & 0.92 & & & & \\
\hline WTC & & & & & & 0.67 & & & & & & & & 1 & & & & 1 & 1 & I & & & & & & 1 & & \\
\hline$B C T$ & & & & & & & & & & & & & & & & & & & & 0.78 & & & & 1 & 1 & & & \\
\hline NWT & & & & & & & & & & & & & & & & & & & & & & & & & & & & \\
\hline PHT & & & & & & & & & & & & & & & & & & & & & & & & & & & & \\
\hline CSP & & & & & & & & & & & & & & & & & & & & & & & & & & & & \\
\hline ANV & & & & & & & & 0.33 & & & & & & & & & & & & 0.69 & & & & & & & & \\
\hline SPR & & & & & & & & 0.39 & & & & & & & & & & & & 0.25 & & & & & & & & \\
\hline$D C P$ & & & & & & & & & & & & & & 1 & & & & 1 & 1 & 1 & & & & & & & & \\
\hline BSZ & & & & & & & & & & & & & & & & & & 1 & 1 & 1 & & & & & & & & \\
\hline BSP & & & & & & & & & & & & & & & & & & & 1 & 1 & & & & & & 1 & & \\
\hline PRV & & & & & & & & & & & & & & & & & & & & & & & & & & & & \\
\hline THR & & & & & & & & & 1 & & & & & & & & & & & & & & & & & & & \\
\hline SMF & & & & & & & & 0.56 & & & & & & & & & & & & 0.72 & & & & & & & & \\
\hline
\end{tabular}

몽 
E4

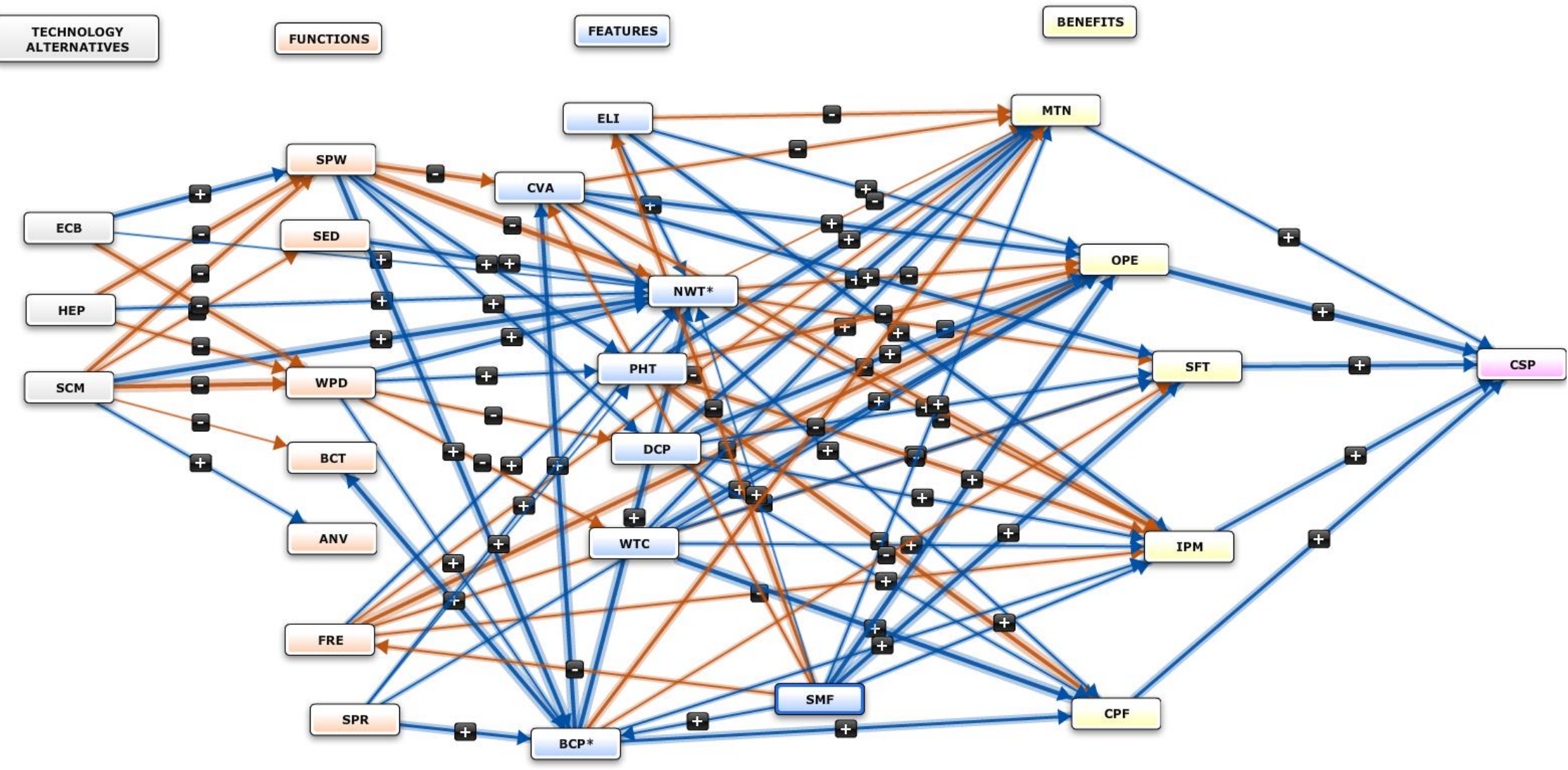




\begin{tabular}{|c|c|c|c|c|c|c|c|c|c|c|c|c|c|c|c|c|c|c|c|c|c|c|c|}
\hline & ELI & FRE & SCM & SED & WPD & CPF & IPM & MTN & SFT & OPE & HEP & ECB & $B C P^{*}$ & SPW & WTC & $\mathrm{BCT}$ & $\mathrm{NWT}^{*}$ & PHT & ANV & CSP & SPR & $\mathrm{DCP}$ & CVA \\
\hline ELI & & & & & & & 0.5 & -0.17 & & 0.38 & & & & & & & 0.17 & & & & & & \\
\hline FRE & & & & & & & -0.37 & -0.29 & -0.42 & -1 & & & & & & & 0.46 & & & & & & \\
\hline SCM & & & & -0.29 & -0.87 & & & & & & & & & -0.62 & & -0.12 & 1 & & 0.46 & & & & \\
\hline SED & & & & & & & & & & & & & & & & & 0.5 & & & & & & \\
\hline WPD & & & & & & & & & & & & & 0.17 & & -0.25 & & 0.58 & 0.38 & & & & -0.25 & \\
\hline CPF & & & & & & & & & & & & & & & & & & & & 0.63 & & & \\
\hline IPM & & & & & & & & & & & & & & & & & & & & 0.79 & & & \\
\hline MTN & & & & & & & & & & & & & & & & & & & & 0.38 & & & \\
\hline SFT & & & & & & & & & & & & & & & & & & & & 0.5 & & & \\
\hline OPE & & & & & & & & & & & & & & & & & & & & 1 & & & \\
\hline HEP & & & & & -0.21 & & & & & & & & & -0.83 & & & 0.17 & & & & & & \\
\hline $\mathrm{ECB}$ & & & & & -0.71 & & & & & & & & & 0.5 & & & 0.08 & & & & & & \\
\hline $\mathrm{BCP}$ * & & & & & & 0.75 & 0.25 & -0.83 & -0.21 & & & & & & & 1 & 1 & & & & & & 1 \\
\hline SPW & & & & & & & & & & & & & 1 & & & & -0.92 & 0.67 & & & & 0.75 & -0.5 \\
\hline WTC & & & & & & 1 & 0.17 & 0.5 & 0.25 & 1 & & & & & & & & & & & & & \\
\hline \multicolumn{24}{|l|}{ BCT } \\
\hline NWT* & & & & & & & -0.17 & -0.12 & -0.25 & -0.17 & & & & & & & & & & & & & \\
\hline PHT & & & & & & -1 & -0.58 & 1 & & -0.83 & & & & & & & & & & & & & \\
\hline \multicolumn{24}{|l|}{$\begin{array}{l}\text { ANV } \\
\text { CSP }\end{array}$} \\
\hline CSP & & & & & & & & & & & & & & & & & & & & & & & \\
\hline SPR & & & & & & & & & & 0.33 & & & 0.75 & & & & 0.21 & 0.08 & & & & & \\
\hline$D C P$ & & & & & & 0.42 & 0.29 & 0.88 & 0.29 & 1 & & & & & & & & & & & & & \\
\hline CVA & & & & & & 0.42 & -0.75 & -0.25 & 0.83 & 0.71 & & & & & & & & & & & & & \\
\hline
\end{tabular}




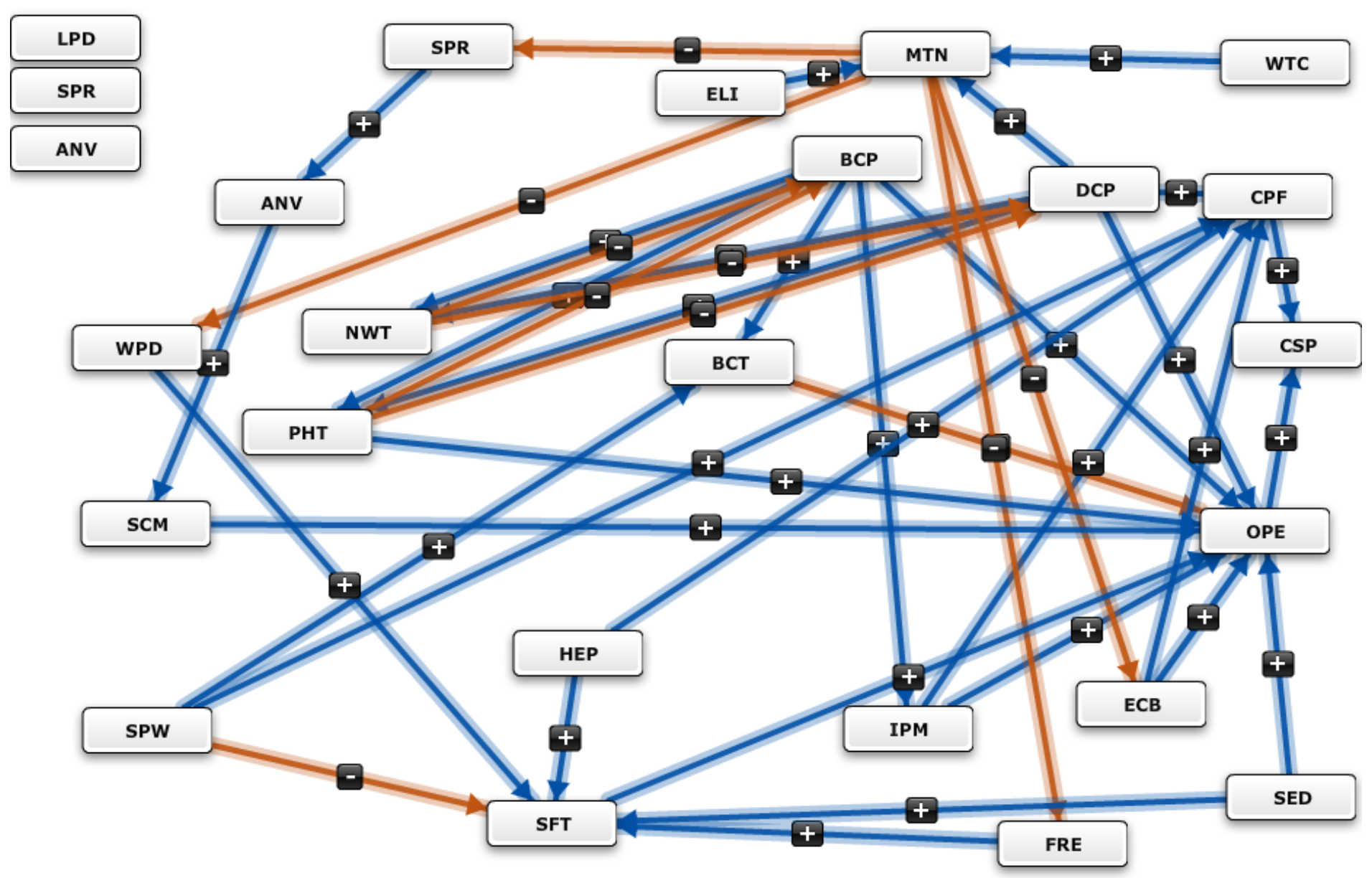




\begin{tabular}{|c|c|c|c|c|c|c|c|c|c|c|c|c|c|c|c|c|c|c|c|c|c|c|c|c|c|}
\hline & ELI & FRE & SCM & SED & WPD & CPF & IPM & MTN & SFT & OPE & HEP & ECB & LPD & $\mathrm{BCP}$ & SPW & WTC & $\mathrm{BCT}$ & NWT & PHT & ANV & SPR & CSP & ANV & SPR & DCP \\
\hline ELI & & & & & & & & 1 & & & & & & & & & & & & & & & & & \\
\hline FRE & & & & & & & & & 1 & & & & & & & & & & & & & & & & \\
\hline SCM & & & & & & & & & & 1 & & & & & & & & & & & & & & & \\
\hline SED & & & & & & & & & 1 & 1 & & & & & & & & & & & & & & & \\
\hline WPD & & & & & & & & & 1 & & & & & & & & & & & & & & & & \\
\hline CPF & & & & & & & & & & & & & & & & & & & & & & 1 & 1 & & \\
\hline IPM & & & & & & 1 & & & & 1 & & & & & & & & & & & & & & & \\
\hline MTN & & -1 & & & -1 & & & & & & & -1 & & & & & & & & & & & & -1 & \\
\hline SFT & & & & & & & & & & 1 & & & & & & & & & & & & & & & \\
\hline OPE & & & & & & & & & & & & & & & & & & & & & & 1 & 1 & & \\
\hline HEP & & & & & & 1 & & & 1 & & & & & & & & & & & & & & & & \\
\hline ECB & & & & & & 1 & & & & 1 & & & & & & & & & & & & & & & \\
\hline LPD & & & & & & & & & & & & & & & & & & & & & & & & & \\
\hline BCP & & & & & & & 1 & & & 1 & & & & & & & 1 & 1 & 1 & 1 & & & & & \\
\hline SPW & & & & & & 1 & & & -1 & & & & & & & & 1 & & & & & & & & \\
\hline WTC & & & & & & & & 1 & & & & & & & & & & & & & & & & & \\
\hline BCT & & & & & & & & & & -1 & & & & & & & & & & & & & & & \\
\hline NWT & & & & & & & & & & & & & & -1 & & & & & & & & & & & -1 \\
\hline PHT & & & & & & & & & & 1 & & & & -1 & & & & & & & & & & & -1 \\
\hline ANV & & & 1 & 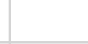 & & & & & & & & & & & & & & & & & & & & & \\
\hline SPR & & & & & & & & & & & & & & & & & & & & & & & & & \\
\hline CSP & & & & & & & & & & & & & & & & & & & & & & & & & \\
\hline ANV & & & & & & & & & & & & & & & & & & & & & & & & & \\
\hline SPR & & & & & & & & & & & & & & & & & & & & 1 & & & & & \\
\hline DCP & & & & & & 1 & & 1 & & 1 & & & & & & & & 1 & 1 & & & & & & \\
\hline
\end{tabular}

$\stackrel{w}{\$}$ 


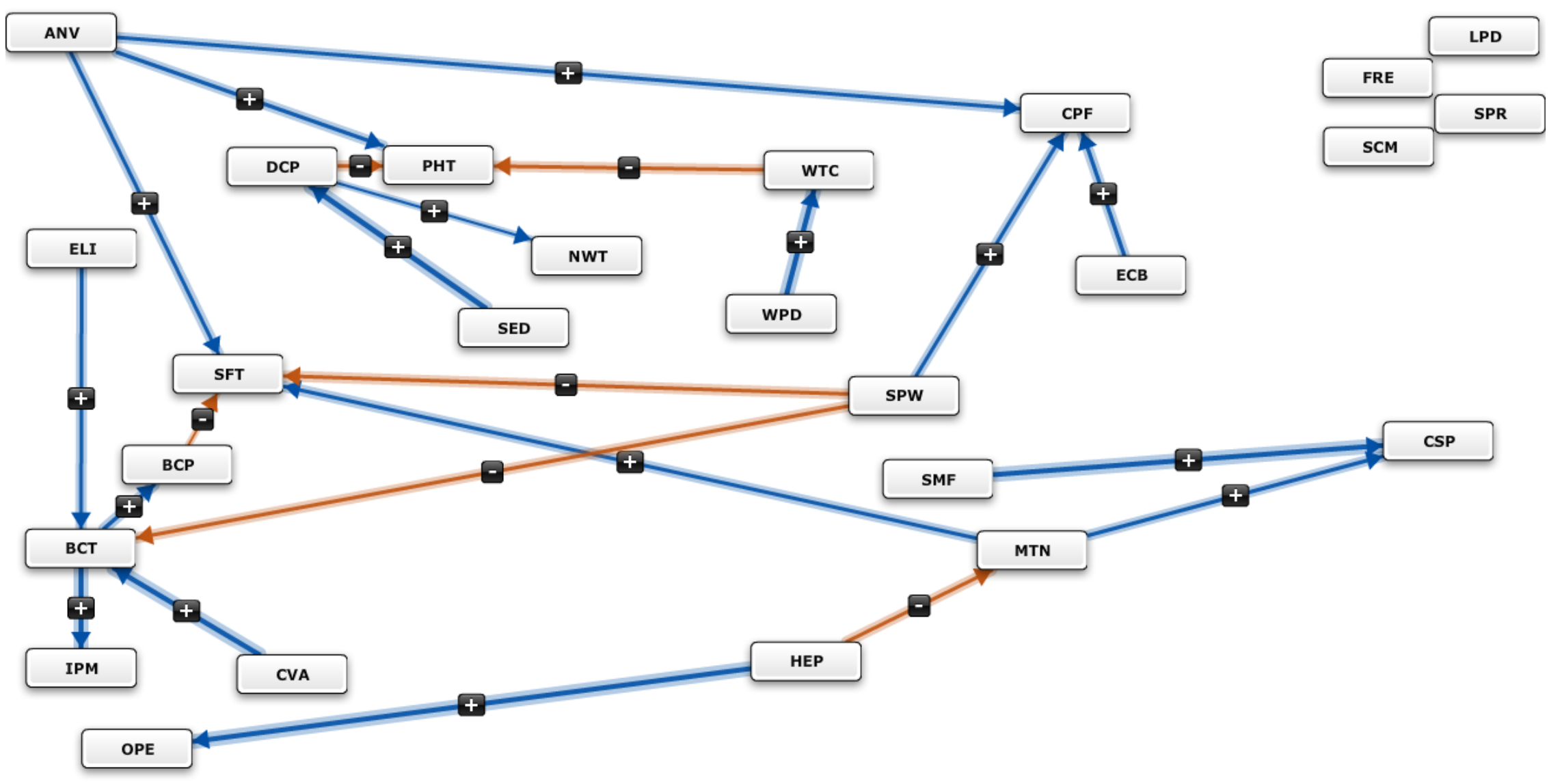

ü 


\begin{tabular}{|c|c|c|c|c|c|c|c|c|c|c|c|c|c|c|c|c|c|c|c|c|c|c|c|c|c|}
\hline & ELI & FRE & SCM & SED & WPD & CPF & IPM & MTN & SFT & OPE & HEP & ECB & LPD & SPW & WTC & BCT & NWT & PHT & CSP & ANV & CVA & DCP & SPR & SMF & BCP \\
\hline ELI & & & & & & & & & & & & & & & & 0.69 & & & & & & & & & \\
\hline FRE & & & & & & & & & & & & & & & & & & & & & & & & & \\
\hline $\mathrm{SCM}$ & & & & & & & & & & & & & & & & & & & & & & & & & \\
\hline SED & & & & & & & & & & & & & & & & & & & & & & 0.87 & & & \\
\hline WPD & & & & & & & & & & & & & & & 0.87 & & & & & & & & & & \\
\hline CPF & & & & & & & & & & & & & & & & & & & & & & & & & \\
\hline IPM & & & & & & & & & & & & & & & & & & & & & & & & & \\
\hline MTN & & & & & & & & & 0.56 & & & & & & & & & & 0.8 & & & & & & \\
\hline SFT & & & & & & & & & & & & & & & & & & & & & & & & & \\
\hline OPE & & & & & & & & & & & & & & & & & & & & & & & & & \\
\hline HEP & & & & & & & & -0.59 & & 0.91 & & & & & & & & & & & & & & & \\
\hline ECB & & & & & & 0.76 & & & & & & & & & & & & & & & & & & & \\
\hline LPD & & & & & & & & & & & & & & & & & & & & & & & & & \\
\hline SPW & & & & & & 0.63 & & & -0.57 & & & & & & & -0.82 & & & & & & & & & \\
\hline WTC & & & & & & & & & & & & & & & & & & -0.8 & & & & & & & \\
\hline BCT & & & & & & & 1 & & & & & & & & & & & & & & & & & & 0.78 \\
\hline NWT & & & & & & & & & & & & & & & & & & & & & & & & & \\
\hline PHT & & & & & & & & & & & & & & & & & & & & & & & & & \\
\hline CSP & & & & & & & & & & & & & & & & & & & & & & & & & \\
\hline ANV & & & & & & 0.67 & & & 0.68 & & & & & & & & & 0.54 & & & & & & & \\
\hline CVA & & & & & & & & & & & & & & & & 0.94 & & & & & & & & & \\
\hline$D C P$ & & & & & & & & & & & & & & & & & 0.46 & -0.8 & & & & & & & \\
\hline SPR & & & & & & & & & & & & & & & & & & & & & & & & & \\
\hline SMF & & & & & & & & & & & & & & & & & & & 1 & & & & & & \\
\hline$B C P$ & & & & & & & & & -0.43 & & & & & & & & & & & & & & & & \\
\hline
\end{tabular}

ő 


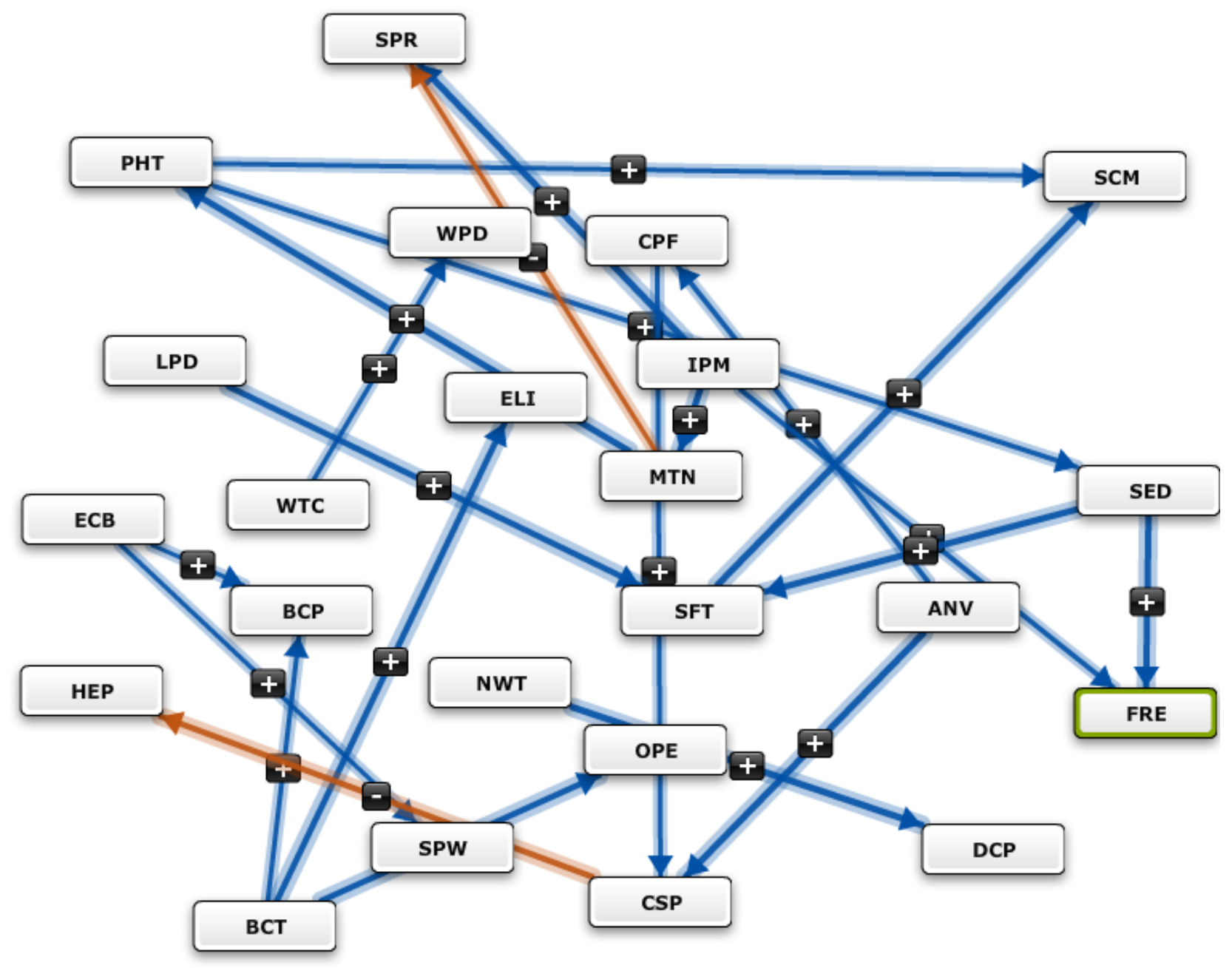




\begin{tabular}{|c|c|c|c|c|c|c|c|c|c|c|c|c|c|c|c|c|c|c|c|c|c|c|c|}
\hline & ELI & FRE & SCM & SED & WPD & $\mathrm{CPF}$ & IPM & MTN & SFT & OPE & HEP & ECB & LPD & BCP & SPW & WTC & BCT & NWT & PHT & CSP & ANV & SPR & DCP \\
\hline \multicolumn{24}{|l|}{ ELI } \\
\hline \multicolumn{24}{|l|}{ FRE } \\
\hline \multicolumn{24}{|l|}{ SCM } \\
\hline SED & & 0.92 & & & & & & & 0.86 & & & & & & & & & & & & & & \\
\hline \multicolumn{24}{|l|}{ WPD } \\
\hline CPF & & & & & & & & & & & & & & & & & & & & 0.83 & & & \\
\hline IPM & & 0.83 & & & & & & 0.89 & & & & & & & & & & & & & & 0.89 & \\
\hline MTN & & & & & & & & & & & & & & & & & & & 0.94 & & & -0.8 & \\
\hline SFT & & & 0.89 & & & & & & & & & & & & & & & & & & & & \\
\hline \multicolumn{24}{|l|}{ OPE } \\
\hline \multicolumn{24}{|l|}{ HEP } \\
\hline ECB & & & & & & & & & & & & & & 0.69 & 0.69 & & & & & & & & \\
\hline LPD & & & & & & & & & 0.94 & & & & & & & & & & & & & & \\
\hline \multicolumn{24}{|l|}{$\mathrm{BCP}$} \\
\hline \multicolumn{24}{|l|}{ SPW } \\
\hline WTC & & & & & 0.58 & & & & & & & & & & & & & & & & & & \\
\hline BCT & 0.86 & & & & & & & & & 0.92 & & & & 0.83 & & & & & & & & & \\
\hline NWT & & & & & & & & & & & & & & & & & & & & & & & 0.86 \\
\hline PHT & & & 0.61 & 0.75 & & & & & & & & & & & & & & & & & & & \\
\hline CSP & & & & & & & & & & & -1 & & & & & & & & & & & & \\
\hline ANV & & & & & & 0.61 & & & & & & & & & & & & & & 0.86 & & & \\
\hline SPR & & & & & & & & & & & & & & & & & & & & & & & \\
\hline DCP & & & & & & & & & & & & & & & & & & & & & & & \\
\hline
\end{tabular}

$\stackrel{w}{\infty}$ 


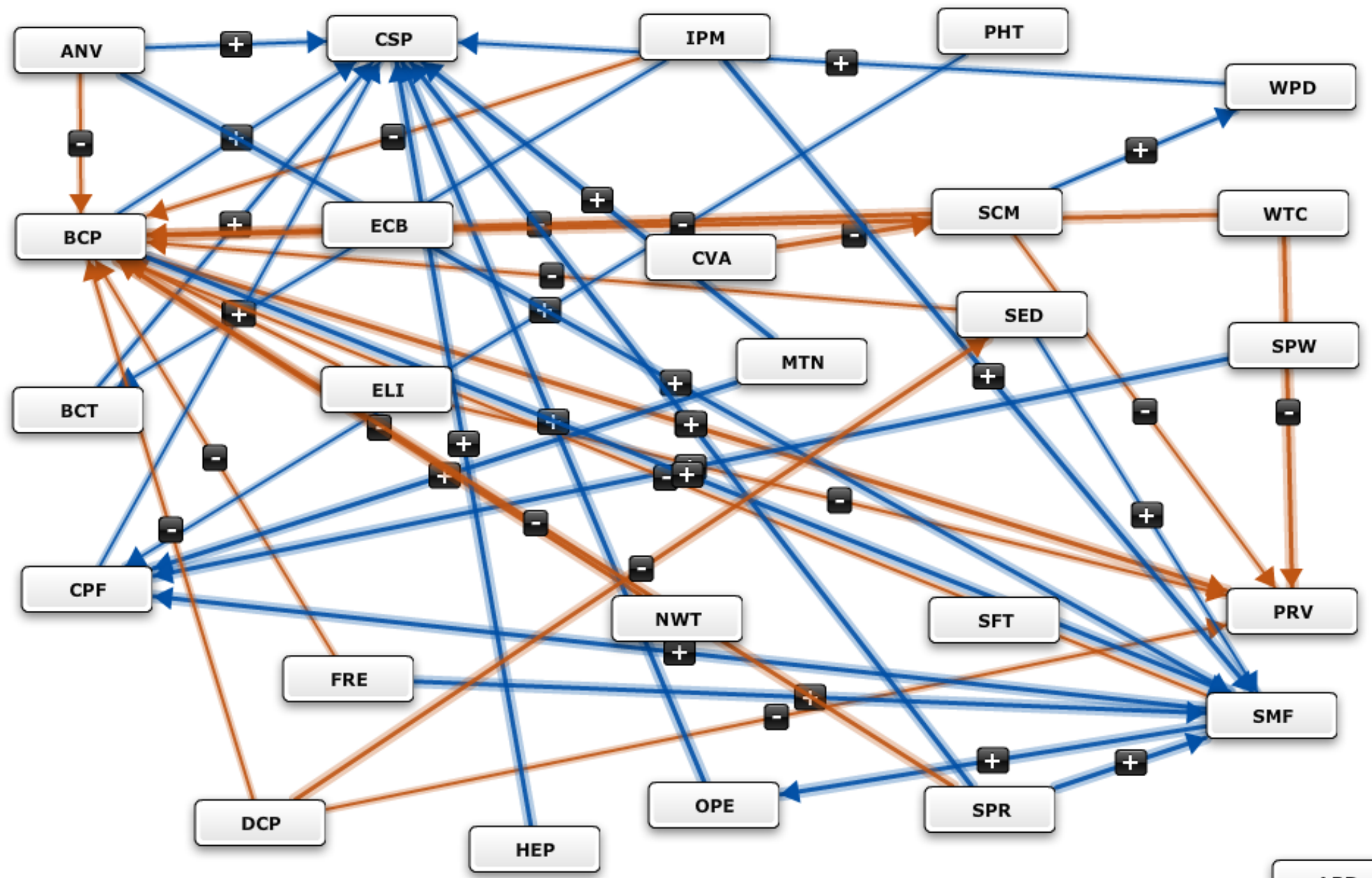




\begin{tabular}{|c|c|c|c|c|c|c|c|c|c|c|c|c|c|c|c|c|c|c|c|c|c|c|c|c|c|c|}
\hline & ELI & FRE & SCM & SED & WPD & CPF & IPM & MTN & SFT & OPE & HEP & $\mathrm{ECB}$ & LPD & $\mathrm{BCP}$ & SPW & WTC & $\mathrm{BCT}$ & NWT & PHT & CSP & ANV & SPR & $\mathrm{DCP}$ & CVA & PRV & SMF \\
\hline ELI & & & & & & & & & & & & & & -0.4 & & & & & & & & & & & -0.4 & \\
\hline FRE & & & & & & & & & & & & & & -0.3 & & & & & & & & & & & & 0.6 \\
\hline $\mathrm{SCM}$ & & & & & 0.44 & & & & & & & & & -0.5 & & & & & & & & & & & -0.4 & \\
\hline SED & & & & & & & & & & & & & & -0.4 & & & & & & & & & & & & 0.44 \\
\hline WPD & & & & & & & & & & & & & & & & & & & & 0.33 & & & & & & \\
\hline CPF & & & & & & & & & & & & & & & & & & & & 0.49 & & & & & & \\
\hline IPM & & & & & & & & & & & & & & -0.3 & & & 0.36 & & & & & & & & & 0.75 \\
\hline MTN & & & & & & 0.69 & & & & & & & & & & & & & & 0.6 & & & & & & \\
\hline SFT & & & & & & & & & & & & & & & & & & & & & & & & & & \\
\hline OPE & & & & & & & & & & & & & & & & & & & & 0.56 & & & & & & \\
\hline HEP & & & & & & & & & & & & & & & & & & & & 0.6 & & & & & & \\
\hline ECB & & & & & & & & & & & & & & & & & & & & & & & & & & \\
\hline LPD & & & & & & & & & & & & & & & & & & & & & & & & & & \\
\hline$B C P$ & & & & & & & & & & & & & & & & & & & & 0.43 & & & & & -0.6 & 0.75 \\
\hline SPW & & & & & & 0.64 & & & & & & & & & & & & & & & & & & & & \\
\hline WTC & & & & & & & & & & & & & & -0.6 & & & & & & & & & & & -0.7 & \\
\hline BCT & & & & & & & & & & & & & & & & & & & & 0.38 & & & & & & \\
\hline NWT & & & & & & & & & & & & & & -0.7 & & & & & & & & & & & & \\
\hline PHT & & & & & & 0.44 & & & & & & & & & & & & & & & & & & & & \\
\hline CSP & & & & & & & & & & & & & & & & & & & & & & & & & & \\
\hline ANV & & & & & & & & & & & & & & -0.5 & & & & & & 0.44 & & & & & & 0.65 \\
\hline SPR & & & & & & & & & & & & & & -0.6 & & & & & & 0.51 & & & & & & 0.61 \\
\hline$D C P$ & & & & -0.6 & & & & & & & & & & -0.4 & & & & & & & & & & & -0.4 & \\
\hline CVA & & & -0.8 & & & & & & & & & & & & & & & & & & & & & & & \\
\hline PRV & & & & & & & & & & & & & & & & & & & & & & & & & & \\
\hline SMF & & & & & & 0.6 & & & & 0.54 & & & & -0.4 & & & & & & & & & & & & \\
\hline
\end{tabular}

$\stackrel{w}{\circ}$ 
Appendix J - Refined Individual FCMs

E1

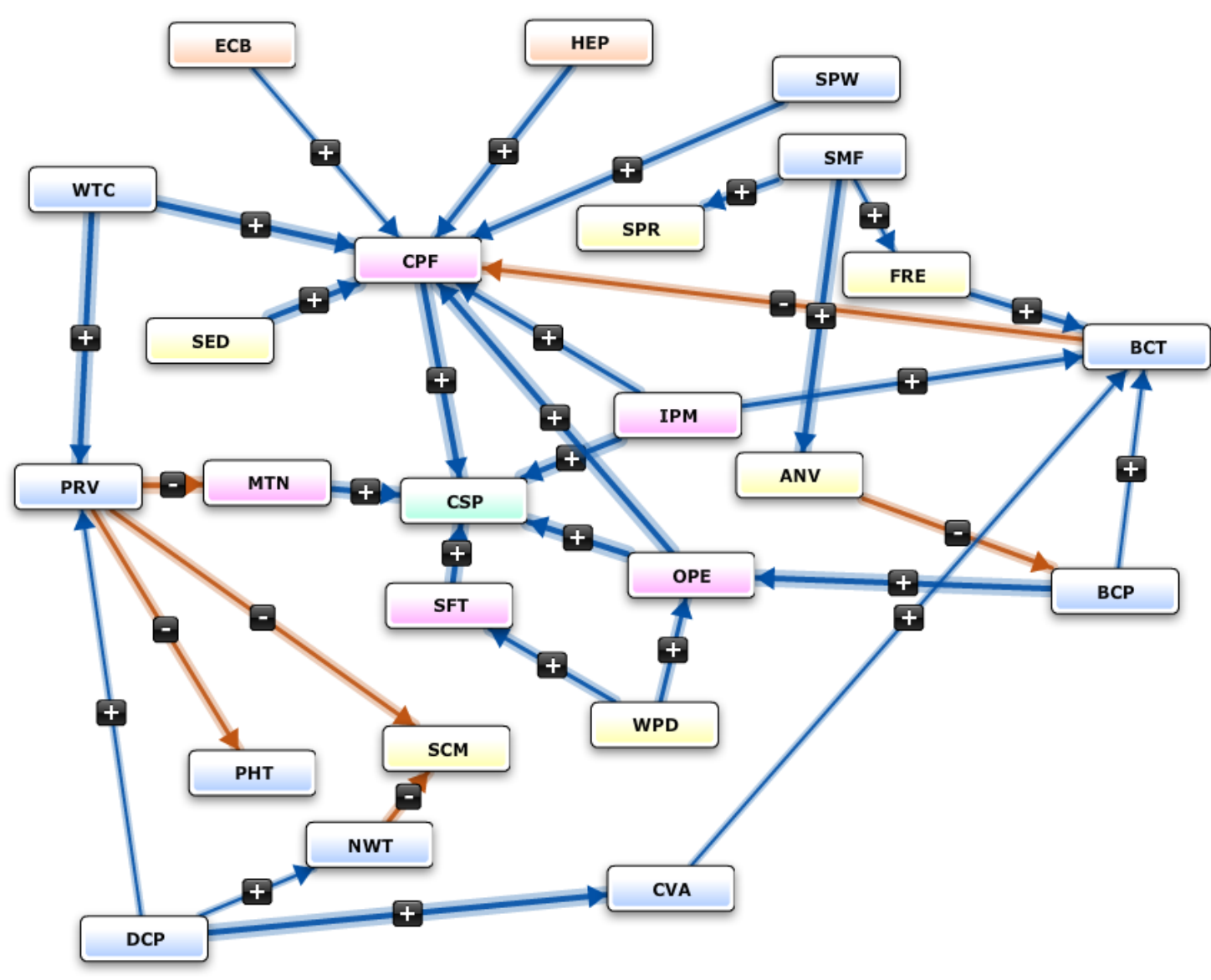




\begin{tabular}{|c|c|c|c|c|c|c|c|c|c|c|c|c|c|c|c|c|c|c|c|c|c|c|c|c|c|c|}
\hline & ELI & FRE & SCM & SED & WPD & CPF & IPM & MTN & SFT & OPE & HEP & $\mathrm{ECB}$ & LPD & $\mathrm{BCP}$ & SPW & WTC & BCT & NWT & PHT & CSP & ANV & SPR & DCP & SMF & PRV & CVA \\
\hline ELI & & & & & & & & & & & & & & & & & & & & 0.43 & & & & & & \\
\hline FRE & & & & & & & & & & & & & & & & & 0.73 & & & 1 & & & & & & \\
\hline $\mathrm{SCM}$ & & & & & & & & & & & & & & & & & & & & 0.97 & & & & & & \\
\hline SED & & & & & & 0.87 & & & & & & & & & & & & & & 1 & & & & & & \\
\hline WPD & & & & & & & & & 0.8 & 0.67 & & & & & & & & & & 1 & & & & & & \\
\hline CPF & & & & & & & & & & & & & & & & & & & & 1 & & & & & & \\
\hline IPM & & & & & & 0.7 & & & & & & & & & & & 0.5 & & & 0.9 & & & & & & \\
\hline MTN & & & & & & & & & & & & & & & & & & & & 1 & & & & & & \\
\hline SFT & & & & & & & & & & & & & & & & & & & & 1 & & & & & & \\
\hline OPE & & & & & & 1 & & & & & & & & & & & & & & 1 & & & & & & \\
\hline HEP & & & & & & 0.83 & & & & & & & & & & & & & & 0.78 & & & & & & \\
\hline ECB & & & & & & 0.37 & & & & & & & & & & & & & & 0.63 & & & & & & \\
\hline LPD & & & & & & & & & & & & & & & & & & & & 1 & & & & & -1 & \\
\hline$B C P$ & & & & & & & & & & 1 & & & & & & & 0.37 & & & & & & & & & \\
\hline SPW & & & & & & 0.5 & & & & & & & & & & & & & & 1 & & & & & & \\
\hline WTC & & & & & & 1 & & & & & & & & & & & & & & 1 & & & & & 0.97 & \\
\hline BCT & & & 0 & & & -0.5 & & & & & & & & & & & & & & -0.5 & & & & & & \\
\hline NWT & & & -0.8 & & & & & & & & & & & & & & & & & 1 & & & & & & \\
\hline PHT & & & & & & & & & & & & & & & & & & & & -0.8 & & & & & & \\
\hline CSP & & & & & & & & & & & & & & & & & & & & & & & & & & \\
\hline ANV & & & & & & & & & & & & & & -0.7 & & & -1 & 1 & & & & & & & & \\
\hline SPR & & & & & & & & & & & & & & & & & & & & 0.9 & & & & & & \\
\hline$D C P$ & & & & & & & & & & & & & & & & & & 0.47 & & 1 & & & & & 0.4 & 1 \\
\hline SMF & & 0.4 & 0 & & & & & 0 & & & & & & & & & 1 & 1 & & 0.43 & 1 & 0.53 & & & & \\
\hline PRV & & & -0.8 & & & & & -1 & & & & & & & & & & & -0.8 & -1 & & & & & & \\
\hline CVA & & & & & & & & & & & & & & & & & 0.2 & & & 0.67 & & & & & & \\
\hline
\end{tabular}

$\stackrel{\omega}{\sim}$ 


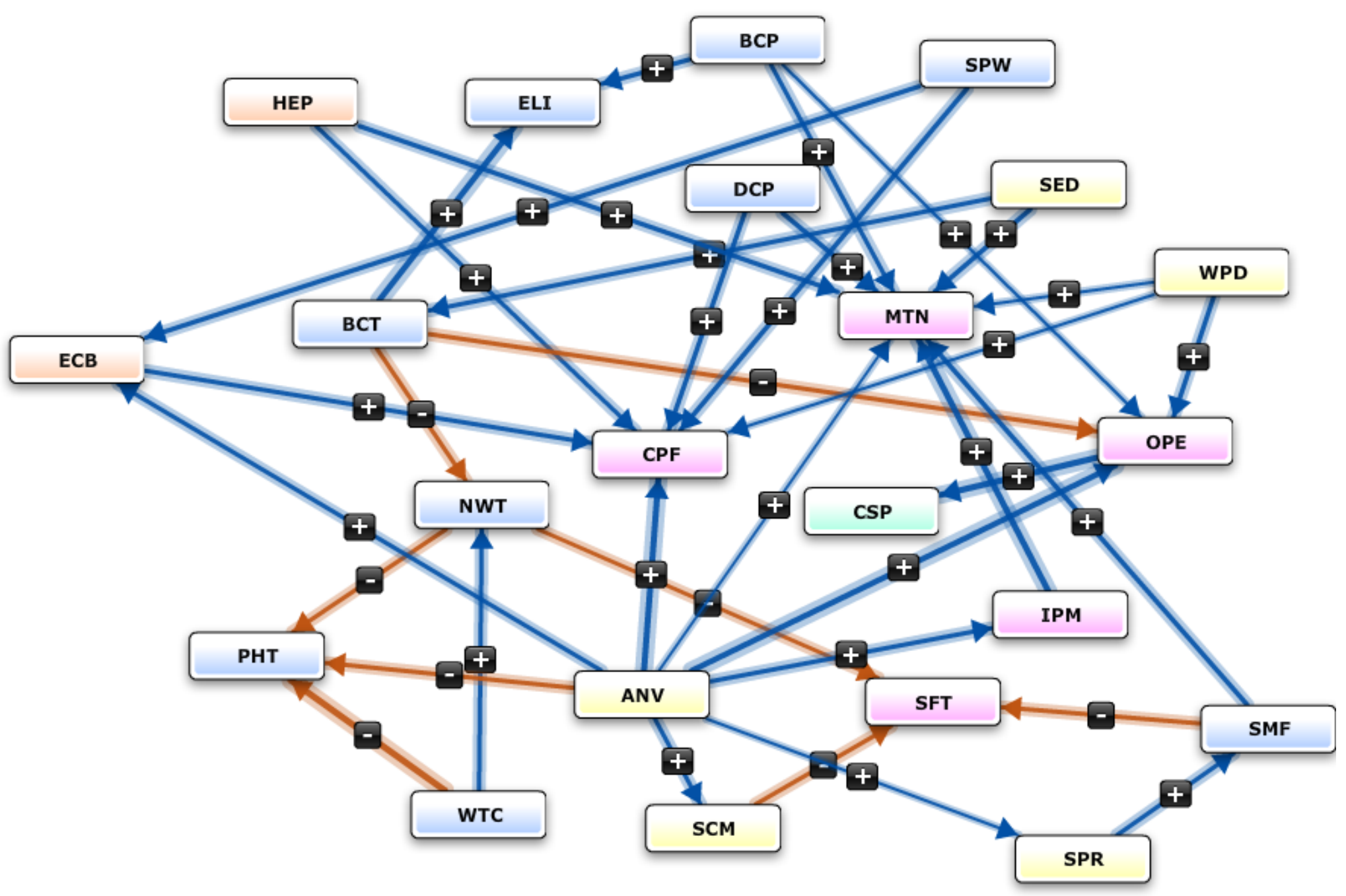




\begin{tabular}{|c|c|c|c|c|c|c|c|c|c|c|c|c|c|c|c|c|c|c|c|c|c|c|c|}
\hline & ELI & $\mathrm{SCM}$ & SED & WPD & CPF & IPM & MTN & SFT & OPE & HEP & $\mathrm{ECB}$ & LPD & $\mathrm{BCP}$ & SPW & WTC & $\mathrm{BCT}$ & NWT & PHT & CSP & ANV & SPR & SMF & $\mathrm{DCP}$ \\
\hline \multicolumn{24}{|l|}{ ELI } \\
\hline SCM & & & & & & & & -0.5 & & & & & & & & & & & & & & & \\
\hline SED & & & & & & & 0.7 & & & & & & & & & 0.75 & & & & & & & \\
\hline WPD & & & & & 0.47 & & 0.47 & & 0.54 & & & & & & & & & & & & & & \\
\hline \multicolumn{24}{|l|}{ CPF } \\
\hline IPM & & & & & & & 0.87 & & & & & & & & & & & & & & & & \\
\hline \multicolumn{24}{|l|}{ MTN } \\
\hline \multicolumn{24}{|l|}{ SFT } \\
\hline OPE & & & & & & & & & & & & & & & & & & & 1 & & & & \\
\hline HEP & & & & & 0.75 & & 0.6 & & & & & & & & & & & & 0.67 & & & & \\
\hline ECB & & & & & 0.77 & & & & & & & & & & & & & & & & & & \\
\hline LPD & & & & & & & & & & & & & & & & & & -1 & & & & & \\
\hline $\mathrm{BCP}$ & 0.77 & & & & & & 0.75 & & 0.49 & & & & & & & & & & & & & & \\
\hline SPW & & & & & 0.7 & & & & & & 0.77 & & & & & & & & 1 & & & & \\
\hline WTC & & & & & & & & & & & & & & & & & 0.67 & -0.9 & & & & & \\
\hline $\mathrm{BCT}$ & 0.93 & & & & & & & & -0.8 & & & & & & & & -0.6 & & 0.6 & & & & \\
\hline NWT & & & & & & & & -0.5 & & & & & & & & & & -0.8 & & & & & \\
\hline \multicolumn{24}{|l|}{ PHT } \\
\hline \multicolumn{24}{|l|}{ CSP } \\
\hline ANV & & 0.6 & & & 1 & 0.55 & 0.49 & & 0.87 & & 0.5 & 0.82 & & & & & & -0.7 & 1 & & 0.44 & & \\
\hline SPR & & & & & & & & & & & & & & & & & & & & & & 0.65 & \\
\hline SMF & & & & & & & 0.67 & -0.6 & & & & & & & & & & & 0.72 & & & & \\
\hline DCP & & & & & 0.7 & & 0.8 & & & & & & & & & & & & 0.77 & & & & \\
\hline
\end{tabular}

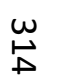




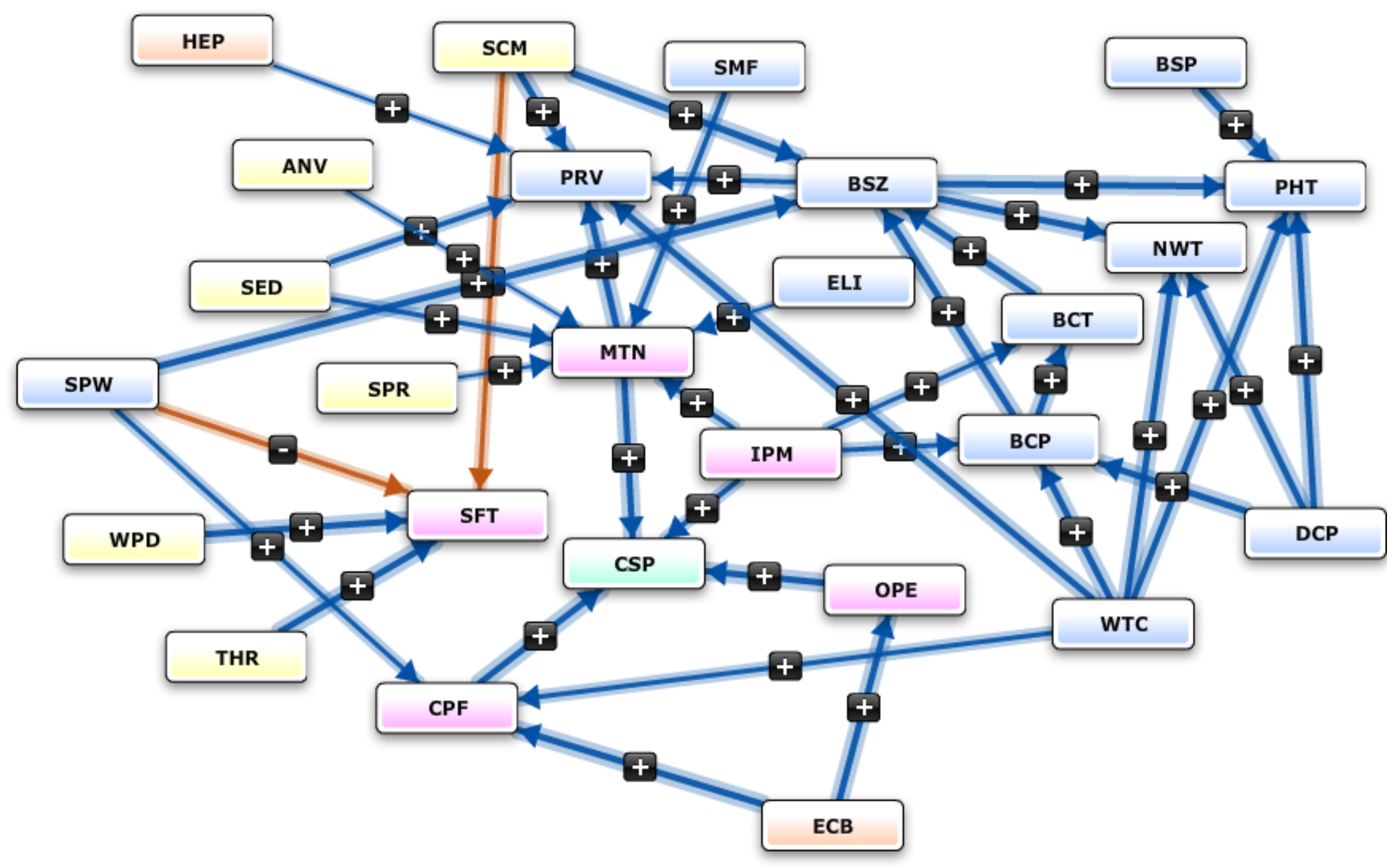




\begin{tabular}{|c|c|c|c|c|c|c|c|c|c|c|c|c|c|c|c|c|c|c|c|c|c|c|c|c|c|c|}
\hline & ELI & SCM & SED & WPD & CPF & IPM & MTN & SFT & HEP & ECB & $B C P$ & SPW & WTC & $\mathrm{BCT}$ & NWT & PHT & CSP & ANV & SPR & SMF & OPE & BSZ & PRV & $\mathrm{DCP}$ & THR & BSP \\
\hline ELI & & & & & & & 0.17 & & & & & & & & & & 1 & & & & & & & & & \\
\hline SCM & & & & & & & & -0.78 & & & & & & & & & & & & & & 1 & 1 & & & \\
\hline SED & & & & & & & 0.83 & & & & & & & & & & 0.5 & & & & & & 0.53 & & & \\
\hline WPD & & & & & & & & 1 & & & & & & & & & & & & & & & & & & \\
\hline CPF & & & & & & & & & & & & & & & & & 1 & & & & & & & & & \\
\hline IPM & & & & & & & 0.53 & & & & 0.69 & & & 0.5 & & & 0.53 & & & & & & & & & \\
\hline MTN & & & & & & & & & & & & & & & & & 1 & & & & & & 1 & & & \\
\hline \multicolumn{27}{|l|}{ SFT } \\
\hline HEP & & & & & & & & & & & & & & & & & 0.81 & & & & & & 0.47 & & & \\
\hline ECB & & & & & 1 & & & & & & & & & & & & 1 & & & & 1 & & & & & \\
\hline$B C P$ & & & & & & & & & & & & & & 1 & & & 0.86 & & & & & 1 & & & & \\
\hline SPW & & & & & 0.81 & & & -0.5 & & & & & & & & & & & & & & 0.92 & & & & \\
\hline WTC & & & & & 0.67 & & & & & & 1 & & & & 1 & 1 & & & & & & & 1 & & & \\
\hline BCT & & & & & & & & & & & & & & & & & 0.67 & & & & & 1 & & & & \\
\hline \multicolumn{27}{|l|}{ NWT } \\
\hline \multicolumn{27}{|l|}{ PHT } \\
\hline \multicolumn{27}{|l|}{ CSP } \\
\hline ANV & & & & & & & 0.33 & & & & & & & & & & 0.69 & & & & & & & & & \\
\hline SPR & & & & & & & 0.39 & & & & & & & & & & 0.25 & & & & & & & & & \\
\hline SMF & & & & & & & 0.56 & & & & & & & & & & 0.72 & & & & & & & & & \\
\hline OPE & & & & & & & & & & & & & & & & & 1 & & & & & & & & & \\
\hline BSZ & & & & & & & & & & & & & & & 1 & 1 & & & & & & & 0.75 & & & \\
\hline \multicolumn{27}{|l|}{ PRV } \\
\hline DCP & & & & & & & & & & & 1 & & & & 1 & 1 & & & & & & & & & & \\
\hline THR & & & & & & & & 1 & & & & & & & & & & & & & & & & & & \\
\hline BSP & & & & & & & & & & & & & & & & 1 & & & & & & & & & & \\
\hline
\end{tabular}

$\stackrel{\omega}{\sigma}$ 
E4

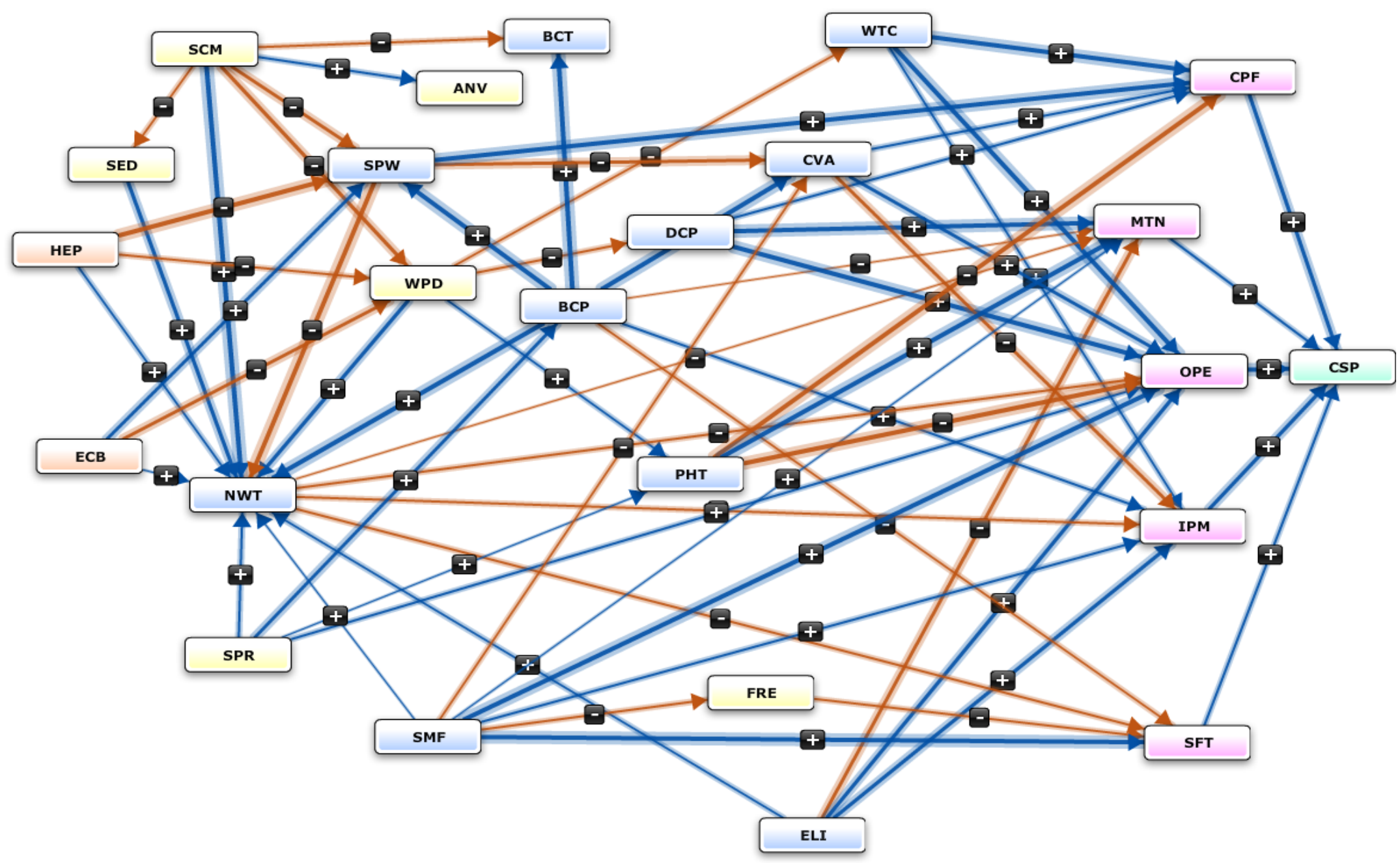




\begin{tabular}{|c|c|c|c|c|c|c|c|c|c|c|c|c|c|c|c|c|c|c|c|c|c|c|c|c|}
\hline & ELI & FRE & SCM & SED & WPD & CPF & IPM & MTN & OPE & HEP & ECB & $\mathrm{BCP}$ & SPW & WTC & $\mathrm{BCT}$ & NWT & CSP & ANV & SPR & DCP & CVA & SMF & SFT & PHT \\
\hline ELI & & & & & & & 0.5 & -0.17 & $\begin{array}{ll}7 & 0.38\end{array}$ & & & & & & & 0.17 & & & & & & & & \\
\hline FRE & & & & & & & -0.37 & $\begin{array}{l}-0.29 \\
\end{array}$ & -1 & & & & & & & 0.46 & & & & & & & -0.42 & \\
\hline SCM & & & & -0.29 & -0.87 & & & & & & & & -0.62 & & -0.12 & 1 & & 0.46 & & & & & & \\
\hline SED & & & & & & & & & & & & & & & & 0.5 & & & & & & & & \\
\hline WPD & & & & & & & & & & & & 0.17 & & -0.25 & & 0.58 & & & & -0.25 & & & & 0.38 \\
\hline CPF & & & & & & & & & & & & & & & & & 0.63 & & & & & & & \\
\hline IPM & & & & & & & & & & & & & & & & & 0.79 & & & & & & & \\
\hline MTN & & & & & & & & & & & & & & & & & 0.38 & & & & & & & \\
\hline OPE & & & & & & & & & & & & & & & & & 1 & & & & & & & \\
\hline HEP & & & & & -0.21 & & & & & & & & -0.83 & & & 0.17 & & & & & & & & \\
\hline ECB & & & & & -0.71 & & & & & & & & 0.5 & & & 0.08 & & & & & & & & \\
\hline$B C P$ & & & & & & 0.75 & 0.25 & -0.83 & & & & & & & 1 & 1 & & & & & 1 & & -0.21 & \\
\hline SPW & & & & & & & & & & & & 1 & & & & -0.92 & & & & 0.75 & -0.5 & & & 0.67 \\
\hline WTC & & & & & & 1 & 0.17 & 0.5 & 1 & & & & & & & & & & & & & & 0.25 & \\
\hline \multicolumn{25}{|l|}{$B C T$} \\
\hline NWT & & & & & & 0 & -0.17 & -0.12 & $\begin{array}{ll}2 & -0.17\end{array}$ & & & & & & & & & & & & & & -0.25 & \\
\hline \multicolumn{25}{|l|}{ CSP } \\
\hline \multicolumn{25}{|l|}{ ANV } \\
\hline SPR & & & & & & & & & 0.33 & & & 0.75 & & & & 0.21 & & & & & & & & 0.08 \\
\hline DCP & & & & & & 0.42 & 0.29 & 0.88 & 1 & & & & & & & & & & & & & & 0.29 & \\
\hline CVA & & & & & & 0.42 & -0.75 & -0.25 & 0.71 & & & & & & & & & & & & & & 0.83 & \\
\hline SMF & -0.74 & -0.44 & & & & & 0.38 & 0.17 & 1 & & & 0.25 & & & & 0.13 & & & & & -0.25 & & 1 & \\
\hline SFT & & & & & & & & & & & & & & & & & 0.5 & & & & & & & \\
\hline PHT & & & & & & -1 & -0.58 & 1 & -0.83 & & & & & & & & & & & & & & & \\
\hline
\end{tabular}

$\underset{\infty}{\omega}$ 


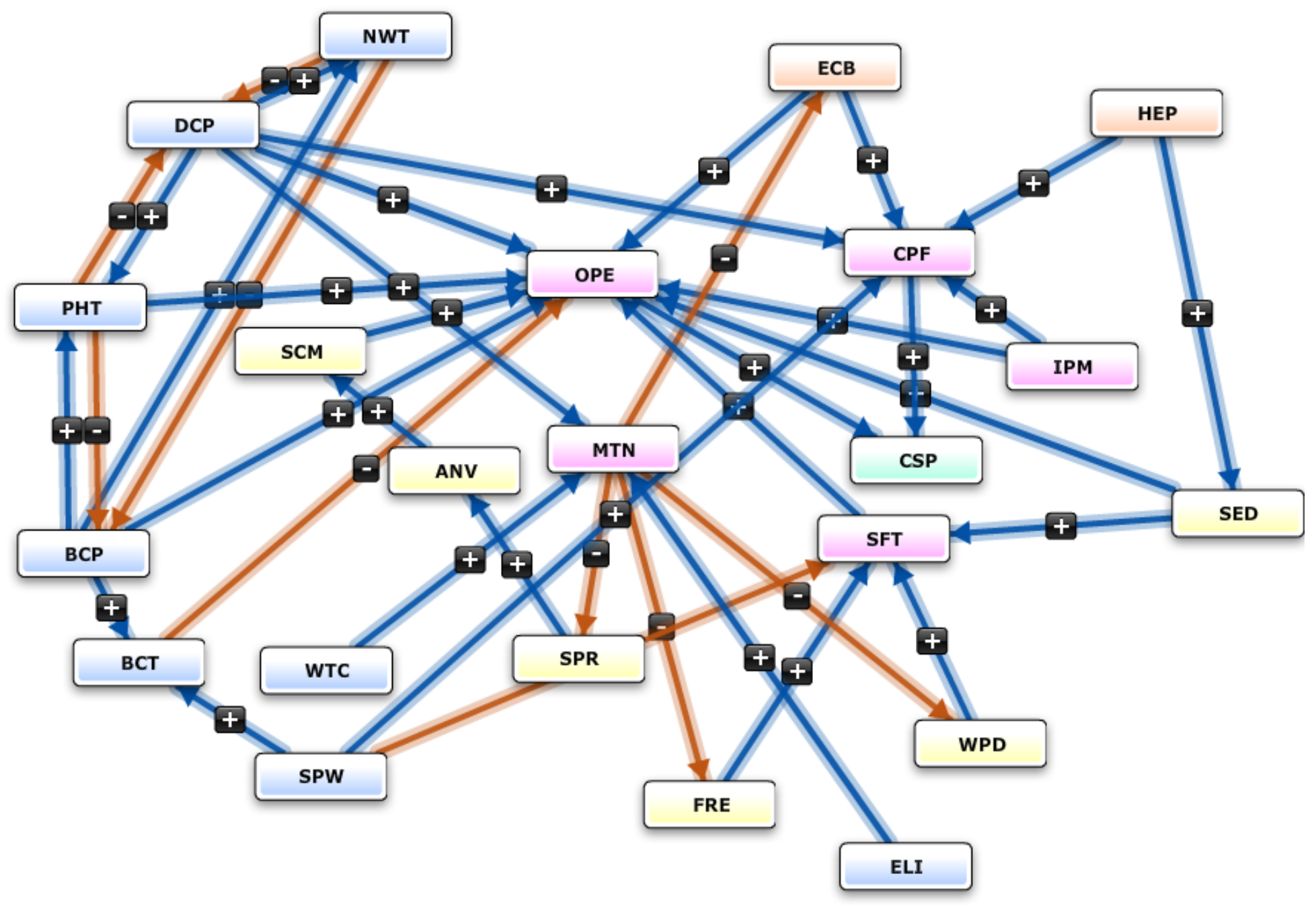




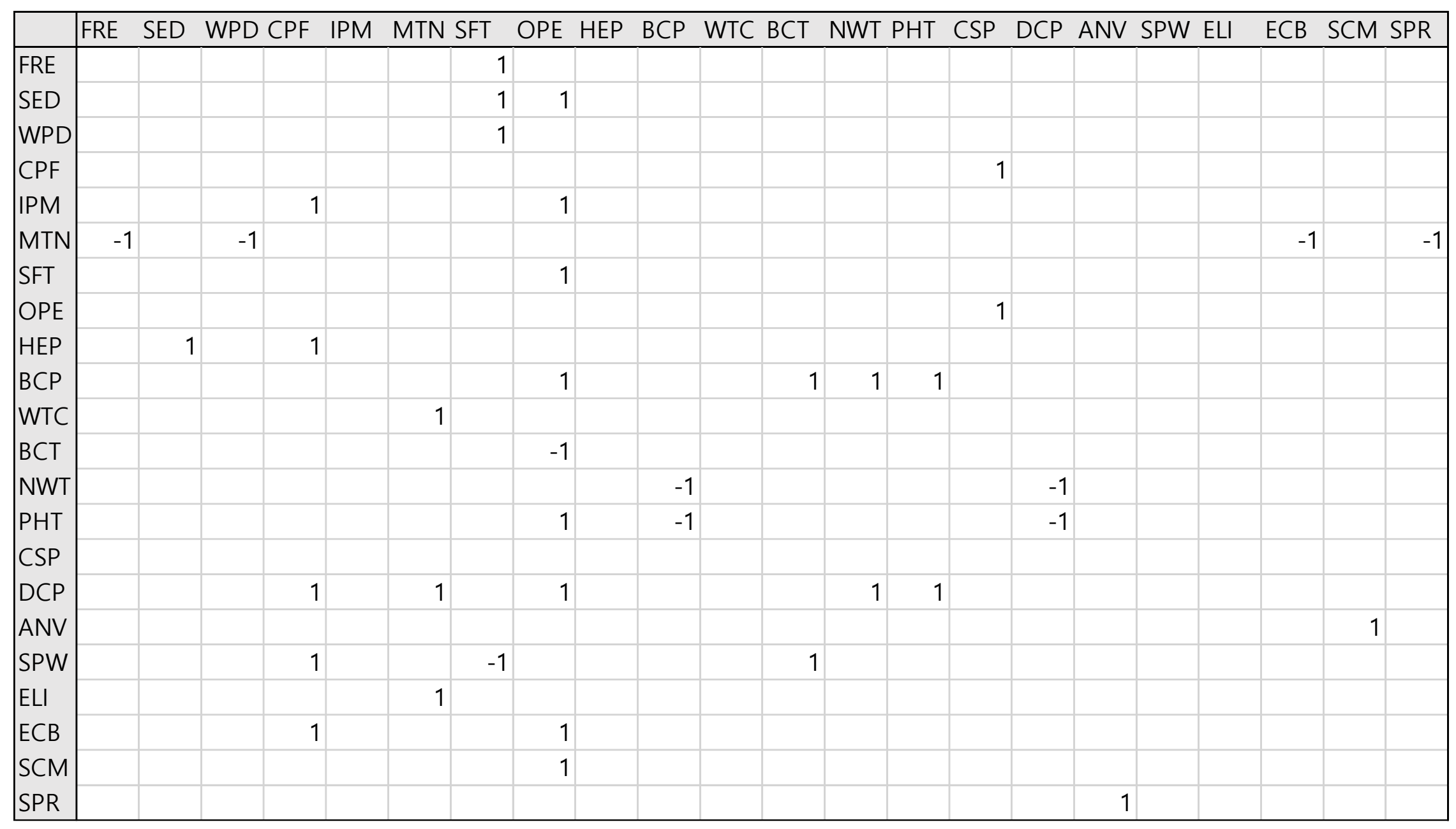

$\stackrel{్}{\sigma}$ 


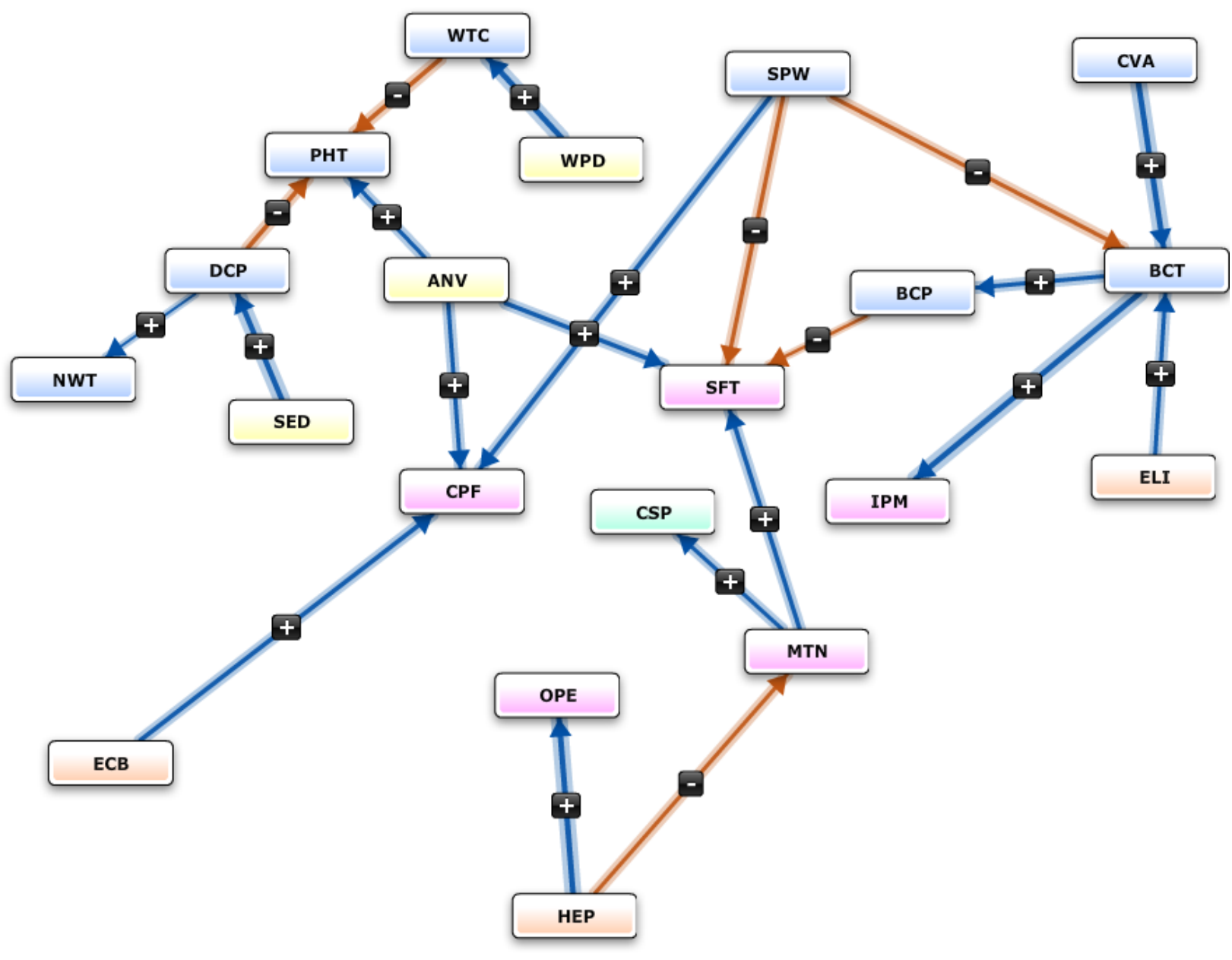




\begin{tabular}{|c|c|c|c|c|c|c|c|c|c|c|c|c|c|c|c|c|c|c|c|c|c|}
\hline & ELI & SED & WPD & CPF & IPM & MTN & SFT & OPE & HEP & $\mathrm{ECB}$ & $B C P$ & SPW & WTC & BCT & NWT & PHT & CSP & ANV & DCP & CVA & SMF \\
\hline ELI & & & & & & & & & & & & & & 0.69 & & & & & & & \\
\hline SED & & & & & & & & & & & & & & & & & & & 0.87 & & \\
\hline WPD & & & & & & & & & & & & & 0.87 & & & & & & & & \\
\hline CPF & & & & & & & & & & & & & & & & & & & & & \\
\hline IPM & & & & & & & & & & & & & & & & & & & & & \\
\hline MTN & & & & & & & 0.56 & & & & & & & & & & 0.8 & & & & \\
\hline SFT & & & & & & & & & & & & & & & & & & & & & \\
\hline OPE & & & & & & & & & & & & & & & & & & & & & \\
\hline HEP & & & & & & -0.6 & & 0.91 & & & & & & & & & & & & & \\
\hline ECB & & & & 0.76 & & & & & & & & & & & & & & & & & \\
\hline BCP & & & & & & & -0.4 & & & & & & & & & & & & & & \\
\hline SPW & & & & 0.63 & & & -0.6 & & & & & & & -0.8 & & & & & & & \\
\hline WTC & & & & & & & & & & & & & & & & -0.8 & & & & & \\
\hline BCT & & & & & 1 & & & & & & 0.78 & & & & & & & & & & \\
\hline NWT & & & & & & & & & & & & & & & & & & & & & \\
\hline PHT & & & & & & & & & & & & & & & & & & & & & \\
\hline CSP & & & & & & & & & & & & & & & & & & & & & \\
\hline ANV & & & & 0.67 & & & 0.68 & & & & & & & & & 0.54 & & & & & \\
\hline DCP & & & & & & & & & & & & & & & 0.46 & -0.8 & & & & & \\
\hline CVA & & & & & & & & & & & & & & 0.94 & & & & & & & \\
\hline SMF & & & & & & & & & & & & & & & & & 1 & & & & \\
\hline
\end{tabular}

$\underset{N}{\sim}$ 


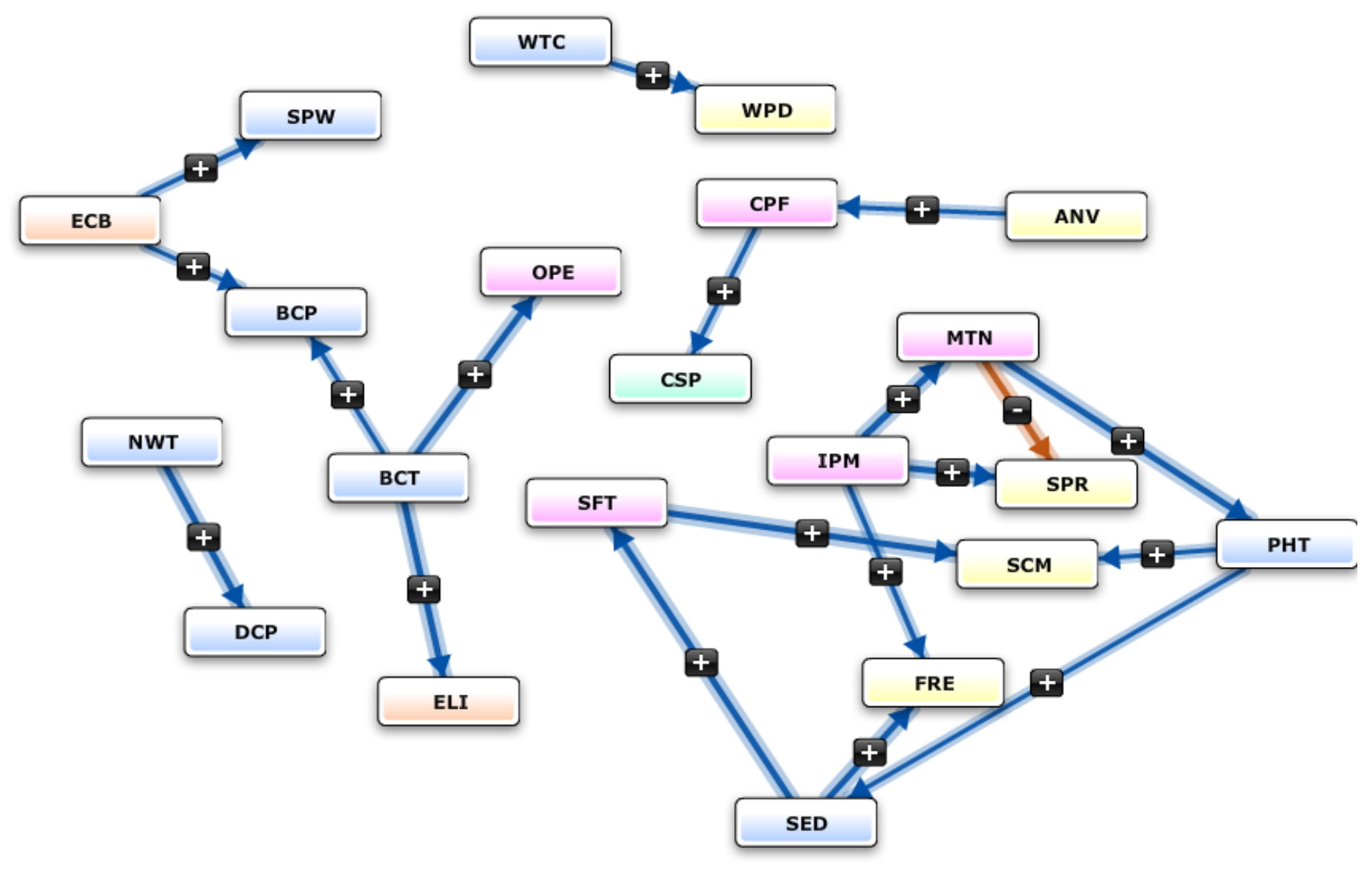




\begin{tabular}{|c|c|c|c|c|c|c|c|c|c|c|c|c|c|c|c|c|c|c|c|c|c|c|}
\hline & ELI & FRE & SCM & SED & WPD & CPF & IPM & MTN & SFT & OPE & ECB & LPD & $B C P$ & SPW & WTC & BCT & NWT & PHT & CSP & ANV & SPR & DCP \\
\hline \multicolumn{23}{|l|}{ ELI } \\
\hline \multicolumn{23}{|l|}{ FRE } \\
\hline \multicolumn{23}{|l|}{ SCM } \\
\hline SED & & 0.91 & & & & & & & 0.85 & & & & & & & & & & & & & \\
\hline \multicolumn{23}{|l|}{ WPD } \\
\hline CPF & & & & & & & & & & & & & & & & & & & 0.82 & & & \\
\hline IPM & & 0.82 & & & & & & 0.88 & & & & & & & & & & & & & 0.88 & \\
\hline MTN & & & & & & & & & & & & & & & & & & 0.94 & & & -0.85 & \\
\hline SFT & & & 0.88 & & & & & & & & & & & & & & & & & & & \\
\hline \multicolumn{23}{|l|}{ OPE } \\
\hline $\mathrm{ECB}$ & & & & & & & & & & & & & 0.69 & 0.69 & & & & & & & & \\
\hline LPD & & & & & & & & & 0.94 & & & & & & & & & & & & & \\
\hline \multicolumn{23}{|l|}{$\mathrm{BCP}$} \\
\hline \multicolumn{23}{|l|}{ SPW } \\
\hline WTC & & & & & 0.57 & & & & & & & & & & & & & & & & & \\
\hline $\mathrm{BCT}$ & 0.85 & & & & & & & & & 0.91 & & & 0.81 & & & & & & & & & \\
\hline NWT & & & & & & & & & & & & & & & & & & & & & & 0.85 \\
\hline PHT & & & 0.6 & 0.75 & & & & & & & & & & & & & & & & & & \\
\hline CSP & & & & & & & & & & & & & & & & & & & & & & \\
\hline ANV & & & & & & 0.6 & & & & & & & & & & & & & 0.85 & & & \\
\hline SPR & & & & & & & & & & & & & & & & & & & & & & \\
\hline DCP & & & & & & & & & & & & & & & & & & & & & & \\
\hline
\end{tabular}

$\underset{\sim}{\sim}$ 


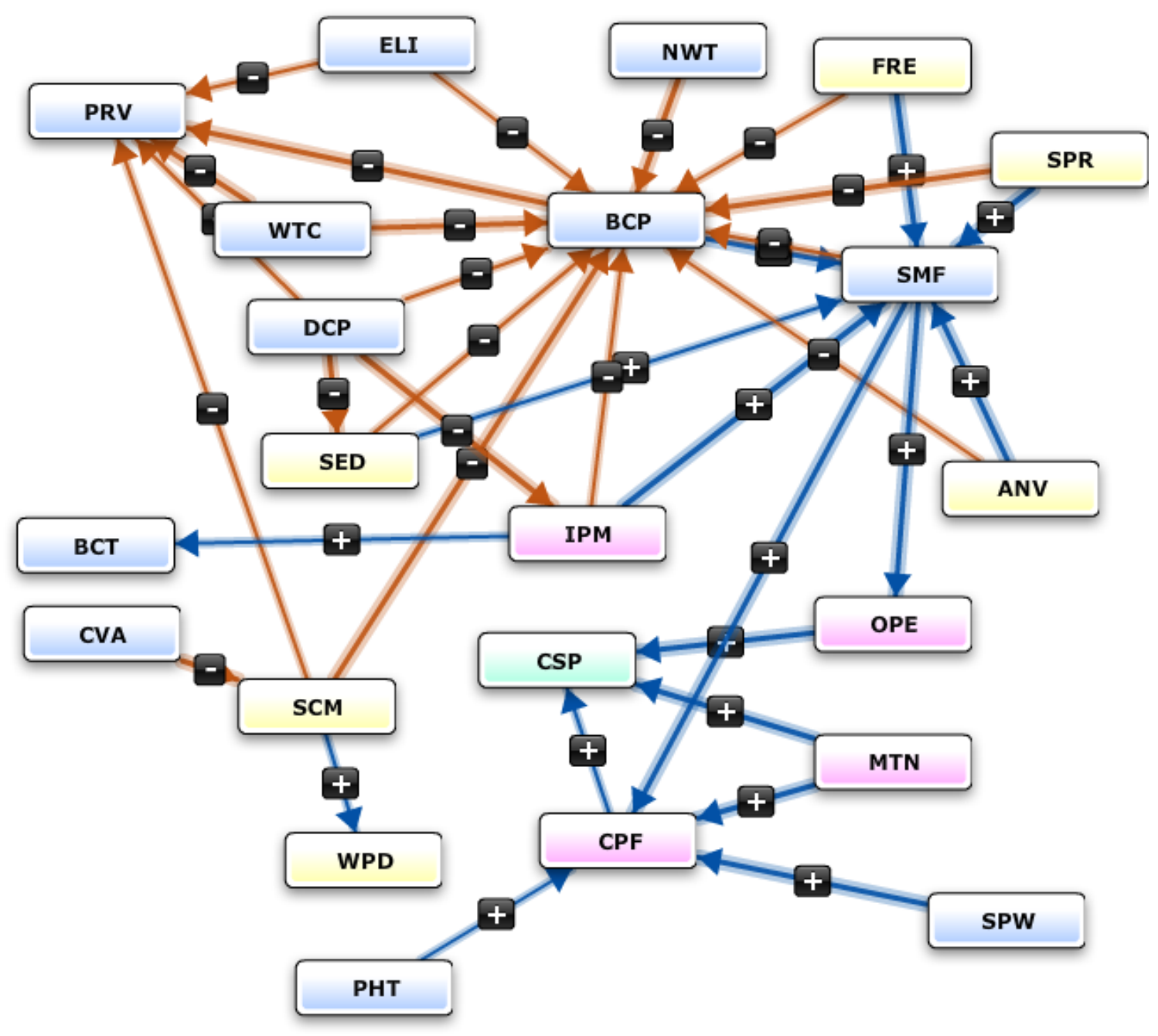




\begin{tabular}{|c|c|c|c|c|c|c|c|c|c|c|c|c|c|c|c|c|c|c|c|c|c|c|c|}
\hline & ELI & FRE & SCM & SED & WPD & CPF & IPM & MTN & OPE & HEP & BCP & SPW & WTC & BCT & NWT & PHT & CSP & ANV & SPR & CVA & SMF & DCP & PRV \\
\hline ELI & & & & & 0 & & & & & & -0.4 & & & & & & & & & & & & -0.4 \\
\hline FRE & & & & & & & & & & & -0.3 & & & & & & & & & & 0.6 & & \\
\hline SCM & & & & & 0.44 & & & & & & -0.5 & & & & & & & & & & & & -0.4 \\
\hline SED & & & & & & & & 0 & & & -0.4 & & & & & & & & & & 0.44 & & \\
\hline WPD & & & & & & & & & & & & & & & & & 0.33 & & & & & & \\
\hline CPF & & & & & & & & & & & & & & & & & 0.49 & & & & & & \\
\hline IPM & & & & & & & & & & & -0.3 & & & 0.36 & & & & & & & 0.75 & & \\
\hline MTN & & & & & & 0.69 & & & & & & & & & & & 0.6 & & & & & & \\
\hline OPE & & & & & & & & & & & & & & & & & 0.56 & & & & & & \\
\hline HEP & & & & & & & & & & & & & & & & & 0.6 & & & & & & \\
\hline $\mathrm{BCP}$ & & & & & & & & & & & & & & & & & 0.43 & & & & 0.75 & & -0.6 \\
\hline SPW & & & & & & 0.64 & & & & & & & & & & & & & & & & & \\
\hline WTC & & & & & & & & & & & -0.6 & & & & & & & & & & & & -0.7 \\
\hline BCT & & & & & & & & & & & & & & & & & 0.38 & & & & & & \\
\hline NWT & & & & & & & & & & & -0.7 & & & & & & & & & & & & \\
\hline PHT & & & & & & 0.44 & & & & & & & & & & & & & & & & & \\
\hline CSP & & & & & & & & & & & & & & & & & & & & & & & \\
\hline ANV & & & & & & & & & & & -0.5 & & & & & & 0.44 & & & & 0.65 & & \\
\hline SPR & & & & & & & & & & & -0.6 & & & & & & 0.51 & & & & 0.61 & & \\
\hline CVA & & & -0.8 & & & & & & & & & & & & & & & & & & & & \\
\hline SMF & & & & & & 0.6 & & & 0.54 & & -0.4 & & & & & & & & & & & & \\
\hline DCP & & & & -0.6 & & & & & & & -0.4 & & & & & & & & & & & & -0.4 \\
\hline PRV & & & & & & & & & & & & & & & & & & & & & & & \\
\hline
\end{tabular}

$\stackrel{\sim}{\sigma}$ 